\title{
Construction and Classification of Symmetry-Protected Topological Phases in Interacting Fermion Systems
}

\author{
Qing-Rui Wang and Zheng-Cheng Gu(** \\ Department of Physics, The Chinese University of Hong Kong, Shatin, New Territories, Hong Kong, China
}

(Received 12 February 2020; revised 20 June 2020; accepted 7 July 2020; published 10 September 2020)

\begin{abstract}
The classification and lattice model construction of symmetry-protected topological (SPT) phases in interacting fermion systems are very interesting but challenging. In this paper, we give a systematic fixedpoint wave function construction of fermionic SPT (FSPT) states for generic fermionic symmetry group $G_{f}=\mathbb{Z}_{2}^{f} \times{ }_{\omega_{2}} G_{b}$ which is a central extension of bosonic symmetry group $G_{b}$ (may contain time-reversal symmetry) by the fermion parity symmetry group $\mathbb{Z}_{2}^{f}=\left\{1, P_{f}\right\}$. Our construction is based on the concept of an equivalence class of finite-depth fermionic symmetric local unitary transformations and decorating symmetry domain wall picture, subjected to certain obstructions. We also discuss the systematical construction and classification of boundary anomalous SPT states which leads to a trivialization of the corresponding bulk FSPT states. Thus, we conjecture that the obstruction-free and trivialization-free constructions naturally lead to a classification of FSPT phases. Each fixed-point wave function admits an exactly solvable commuting-projector Hamiltonian. We believe that our classification scheme can be generalized to point and space group symmetry as well as continuum Lie group symmetry.
\end{abstract}

DOI: $10.1103 /$ PhysRevX.10.031055

Subject Areas: Condensed Matter Physics

\section{INTRODUCTION}

\section{A. The goal of this paper}

Topological phases of quantum matter have become a fascinating subject in the past three decades. The concept of long-range entanglement and an equivalence class of finite-depth local unitary (LU) transformation [1] provides us a paradigm toward classifying and systematically constructing these intriguing quantum states. It was realized that the patterns of long-range entanglement are the essential data to characterize various topological phases of quantum matter.

In recent years, the research on the interplay between topology and symmetry also has achieved a lot of fruitful results. The concept of an equivalence class of finite-depth symmetric LU (SLU) transformations suggests that, in the presence of global symmetry, even short-range entangled (SRE) states still can belong to many different phases if they do not break any symmetry of the system. (It is well known that the traditional Landau symmetry-breaking states are characterized by different broken symmetries.) Thus, these new SRE states of quantum matter are named

\footnotetext{
*zcgu@phy.cuhk.edu.hk
}

Published by the American Physical Society under the terms of the Creative Commons Attribution 4.0 International license. Further distribution of this work must maintain attribution to the author(s) and the published article's title, journal citation, and DOI. as symmetry-protected topological (SPT) phases [2-4]. Topological insulators $[5,6]$ are the simplest examples of SPT phases, which are protected by time-reversal and charge-conservation symmetries.

By definition, all SPT phases can be adiabatically connected to a trivial disorder phase (e.g., a product state or an atomic insulator) in the absence of global symmetry. In Ref. [2], it is first pointed out that the well-known spin-1 Haldane chain [7] is actually an SPT phase which can be adiabatically connected to a trivial disorder phase in the absence of any symmetry. Thus, SPT phases can always be constructed by applying LU transformations onto a trivial product state. Such a special property makes it possible to systematically construct and classify SPT phases for interacting systems. For example, Refs. [3,4] introduce a systematic way of constructing fixed-point partition functions and exactly solvable lattice models for interacting bosonic systems using group cohomology theory, and it has been believed that such a construction is fairly complete for bosonic SPT (BSPT) phases protected by unitary symmetry up to 3D. Physically, the corresponding fixed-point groundstate wave functions of such a construction can be regarded as a superposition of fluctuation symmetry domain walls. Later, it was pointed out that, by further decorating the $E_{8}$ state onto the symmetry domain wall [8], the fluctuation symmetry domain wall picture can actually describe all BSPT phases, which are believed to be classified by cobordism theory $[9,10]$. In Sec. II, we review how to use the equivalence class of finite-depth SLU transformation 
approach and fluctuation symmetry domain wall picture to classify and construct all BSPT phases with unitary symmetries up to 3D.

Although the SRE SPT phases seem to be not as interesting as long-range entangled topological phases due to the absence of bulk fractionalized excitations, the concept of "gauging" the global symmetry of SPT phases establishes a direct mapping from SPT phases to intrinsic topological phases. In fact, it has been shown that different BSPT phases protected by a unitary symmetry group $G$ can be characterized by different types of braiding statistics of $G$ flux in 2D and different types of the so-called three-loop braiding statistics of flux lines in 3D [11-22]. Very recently, it has been further conjectured that all topological phases in 3D interacting systems can actually be realized by "gauging" certain SPT phases [23,24].

Moreover, the classification of SPT phases in interacting systems turns out to be a one-to-one correspondence with the classification of global anomalies on the boundary [25]. For example, anomalous surface topological order is proposed as another very powerful way to identify and characterize different 3D SPT phases in interacting systems [26-37]. In high-energy physics, it is well known that global anomalies can be characterized and classified by cobordism (spin cobordism) for interacting boson (fermion) systems; thus, it is not a surprise that the classification of SPT phases is closely related to cobordism (spin cobordism) theory [10,20,21].

Despite the fact that great success has been made on the construction and classification of SPT phases in interacting boson systems and free-fermion systems, understanding of SPT phases in interacting fermion systems is still very limited, especially on the construction of microscopic models. Previously, a lot of effort has been made on the reduction of the free-fermion classifications [38-41] under the effect of interactions [42-49]. On the other hand, stacking BSPT states onto free-fermion SPT states is another obvious way to generate some new SPT phases $[34,50]$. Apparently, these two approaches miss those fermionic SPT (FSPT) phases that can be realized neither in free-fermion systems nor in interacting bosonic systems $[13,51]$. Moreover, it has been further shown that certain BSPT phases become "trivial" (adiabatically connected to a product state) $[34,52]$ when embedded into interacting fermion systems. Therefore, a systematical understanding for the classification and construction of SPT phases in interacting fermion systems is very desired.

Very recently, based on the concept of an equivalence class of finite-depth fermionic SLU (FSLU) transformation and decorated symmetry domain wall picture, a breakthrough has been made on the full construction and classification of FSPT states with a total symmetry $G_{f}=$ $G_{b} \times \mathbb{Z}_{2}^{f}$ (where $G_{b}$ is the bosonic unitary symmetry and $\mathbb{Z}_{2}^{f}$ is the fermion parity conservation symmetry) [53]. The fixed-point wave functions generated by FSLU transformations can be realized by exactly solvable lattice models, and the resulting classification results all agree with previous studies in 1D and 2D using other methods [43,52,54-57]. Most surprisingly, such a completely different physical approach precisely matches the potential global anomaly for interacting fermion systems classified by spin cobordism theory [10,58-64].

It turns out that the mathematical objects that classify 1D FSPT phases with a total symmetry $G_{f}=G_{b} \times \mathbb{Z}_{2}^{f}$ can be summarized as two cohomology groups of the symmetry group $G_{b}: H^{1}\left(G_{b}, \mathbb{Z}_{2}\right)$ and $H^{2}\left[G_{b}, U(1)\right]$, which correspond to the complex fermion decoration on $G_{b}$ symmetry domain walls and classification of 1D BSPT phases.

The mathematical objects that classify 2D FSPT phases with a total symmetry $G_{f}=G_{b} \times \mathbb{Z}_{2}^{f}$ are slightly complicated and can be summarized as three cohomology groups of the symmetry group $G_{b}[55,59]: H^{1}\left(G_{b}, \mathbb{Z}_{2}\right), B H^{2}\left(G_{b}, \mathbb{Z}_{2}\right)$, and $H^{3}\left[G_{b}, U(1)\right] . H^{1}\left(G_{b}, \mathbb{Z}_{2}\right)$ corresponds to the Majorana chain decoration on $G_{b}$ symmetry domain walls. Naively, one may expect that the complex fermion decorations on the intersection point of $G_{b}$ symmetry domain walls should be described by the data $H^{2}\left(G_{b}, \mathbb{Z}_{2}\right)$. However, it turns out that such a decoration scheme suffers from obstructions, and only the subgroup $B H^{2}\left(G_{b}, \mathbb{Z}_{2}\right)$ classifies valid and inequivalent $2 \mathrm{D}$ FSPT phases. More precisely, $B H^{2}\left(G_{b}, \mathbb{Z}_{2}\right)$ is defined by $n_{2} \in H^{2}\left(G_{b}, \mathbb{Z}_{2}\right)$ that satisfy $S q^{2}\left(n_{2}\right)=0$ in $H^{4}\left[G_{b}, U(1)\right]$, where $S q^{2}$ is the Steenrod square operation, $S q^{2}: H^{d}\left(G_{b}, \mathbb{Z}_{2}\right) \rightarrow H^{d+2}\left(G_{b}, \mathbb{Z}_{2}\right)$ [65]. Finally, $H^{3}\left[G_{b}, U(1)\right]$ is the well-known classification of BSPT phases.

Similarly, the mathematical objects that classify 3D FSPT phases with a total symmetry $G_{f}=G_{b} \times \mathbb{Z}_{2}^{f}$ can also be summarized as three cohomology groups of the symmetry group $G_{b}[53,62]: \tilde{B} H^{2}\left(G_{b}, \mathbb{Z}_{2}\right), B H^{3}\left(G_{b}, \mathbb{Z}_{2}\right)$, and $H_{\text {rigid }}^{4}\left[G_{b}, U(1)\right]$. As a subgroup of $H^{2}\left(G_{b}, \mathbb{Z}_{2}\right)$, $\tilde{B} H^{2}\left(G_{b}, \mathbb{Z}_{2}\right)$ corresponds to the Majorana chain decoration on the intersection lines of $G_{b}$ symmetry domain walls subject to much more subtle and complicated objections related to discrete spin structure. Again, as a subgroup of $H^{3}\left(G_{b}, \mathbb{Z}_{2}\right), B H^{3}\left(G_{b}, \mathbb{Z}_{2}\right)$ corresponds to the complex fermion decoration on the intersection points of $G_{b}$ symmetry domain walls. And it is formed by elements $n_{3} \in$ $H^{3}\left(G_{b}, \mathbb{Z}_{2}\right)$ that satisfy $S q^{2}\left(n_{3}\right)=0$ in $H^{4}\left[G_{b}, U(1)\right]$. Finally, $H_{\text {rigid }}^{4}\left[G_{b}, U(1)\right] \equiv H^{4}\left[G_{b}, U(1)\right] / \Gamma^{4}$ corresponds to stable BSPT phases when embedded into interacting fermion systems. We note that $\Gamma^{4}$ is a normal subgroup of $H^{4}\left[G_{b}, U(1)\right]$ generated by $(-)^{S q^{2}\left(n_{2}\right)}$, where $n_{2} \in$ $H^{2}\left(G_{b}, \mathbb{Z}_{2}\right)$ and $(-)^{S q^{2}\left(n_{2}\right)}$ are viewed as elements of $H^{4}\left[G_{b}, U(1)\right]$. Physically, $\Gamma^{4}$ corresponds to those trivialized BSPT phases when embedded into interacting fermion systems.

In this paper, we aim to generalize the above constructions and classifications of FSPT phases to generic 
fermionic symmetry group $G_{f}=\mathbb{Z}_{2}^{f} \times_{\omega_{2}} G_{b}$, which is a central extension of bosonic symmetry group $G_{b}$ (may contain time-reversal symmetry) by the fermion parity symmetry group $\mathbb{Z}_{2}^{f}=\left\{1, P_{f}\right\}$. We show that the equivalence class of finite-depth FSLU transformation and decorated symmetry domain wall picture still applies for generic cases, subjected to much more complicated obstruction conditions. Moreover, we also clarify the physical meaning of obstruction by introducing the notion of anomalous SPT (ASPT) states [66], that is, a new kind of SPT state that can be realized only on the boundary of certain SPT states in one dimension higher. Meanwhile, this notion also implies that the corresponding bulk SPT states are actually trivialized. Finally, we show that, if $G_{b}$ is time-reversal symmetry, an additional layer of $p+i p$ topological superconducting state decoration on the symmetry domain wall leads to new FSPT states, which is the analogy of decorating the $E_{8}$ state onto the symmetry domain wall for BSPT phases with time-reversal symmetry [8].

\section{B. Some generalities of fermionic symmetry groups}

For a fermionic system with total symmetry group $G_{f}$, there is always a subgroup: the fermion parity symmetry group $\mathbb{Z}_{2}^{f}=\left\{1, P_{f}=(-1)^{F}\right\}$, where $F$ is the total fermion number operator. The subgroup $\mathbb{Z}_{2}^{f}$ is in the center of $G_{f}$, because all physical symmetries should not change the fermion parity of the state, i.e., commute with $P_{f}$. Therefore, we can construct a quotient group $G_{b}=G_{f} / \mathbb{Z}_{2}^{f}$, which we call the bosonic symmetry group.

Conversely, for a given bosonic symmetry group $G_{b}$, there are many different fermionic symmetry groups $G_{f}$, which is the central extension of $G_{b}$ by $\mathbb{Z}_{2}^{f}$. We have the following short exact sequence:

$$
1 \rightarrow \mathbb{Z}_{2}^{f} \rightarrow G_{f} \rightarrow G_{b} \rightarrow 1 .
$$

Different extensions $G_{f}$ are specified by 2-cocycles $\omega_{2} \in H^{2}\left(G_{b}, \mathbb{Z}_{2}=\{0,1\}\right)$. This result is the reason why we denote $G_{f}$ as $\mathbb{Z}_{2}^{f} \times{ }_{\omega_{2}} G_{b}$. The group element $g_{f}$ of $G_{f}$ has the form $g_{f}=\left(P_{f}^{n(g)}, g_{b}\right) \in \mathbb{Z}_{2}^{f} \times G_{b}$, with $n(g)=0,1$. We may also simply denote it as $g_{f}=P_{f}^{n(g)} g_{b}$. And the multiplication rule in $G_{f}$ is given by

$$
\begin{aligned}
g_{f} \cdot h_{f} & =\left(P_{f}^{n(g)}, g_{b}\right) \cdot\left(P_{f}^{n(h)}, h_{b}\right) \\
& :=\left(P_{f}^{n(g)+n(h)+\omega_{2}\left(g_{b}, h_{b}\right)}, g_{b} h_{b}\right),
\end{aligned}
$$

where we have $P_{f}^{n(g)+n(h)+\omega_{2}\left(g_{b}, h_{b}\right)} \in \mathbb{Z}_{2}^{f}$ and $g_{b} h_{b} \in G_{b}$. The associativity condition of $g \cdot h \cdot k\left(g, h, k \in G_{b}\right)$ gives rise to the cocycle equation for $\omega_{2}$ :

$$
\begin{aligned}
\left(d \omega_{2}\right)(g, h, k):= & \omega_{2}(h, k)+\omega_{2}(g h, k)+\omega_{2}(g, h k) \\
& +\omega_{2}(g, h)=0 \quad(\bmod 2) .
\end{aligned}
$$

We omit the subscript of $g_{b}$ and use merely $g$ to denote the group element of $G_{b}$ henceforth. One can also show that adding coboundaries to $\omega_{2}$ gives rise to isomorphic $G_{f}$. Therefore, $\omega_{2}$ is an element in $H^{2}\left(G_{b}, \mathbb{Z}_{2}\right)$ and classifies the central extension of $G_{b}$ by $\mathbb{Z}_{2}^{f}$. Note that there is another constraint for $\omega_{2}$ as $\omega_{2}(e, g)=\omega_{2}(g, e)=0$ ( $e$ is the identity element of $G_{b}$ ).

Another ingredient of the symmetry group is associated with time-reversal symmetry which is antiunitary. We can use a function $s_{1}$ with

$$
s_{1}(g)= \begin{cases}0, & g \text { is unitary, } \\ 1, & g \text { is antiunitary }\end{cases}
$$

to indicate whether $g \in G_{b}$ is antiunitary or not. The function $s_{1}$ is a group homomorphism from $G_{b}$ to $\mathbb{Z}_{2}$ because of the property

$$
\left(d s_{1}\right)(g, h):=s_{1}(h)+s_{1}(g h)+s_{1}(g)=0(\bmod 2) .
$$

So $s_{1}$ can also be viewed as a 1-cocycle in $H^{1}\left(G_{b}, \mathbb{Z}_{2}\right)$.

Let us consider some examples. The superconductor with time-reversal symmetry $T^{2}=P_{f}\left(T^{2}=-1\right.$ when acting on single-fermion states) has bosonic symmetry group $G_{b}=\mathbb{Z}_{2}^{T}=\{e, T\}$ and fermionic symmetry group $G_{f}=\mathbb{Z}_{4}^{T f}=\mathbb{Z}_{2}^{f} \times_{\omega_{2}} \mathbb{Z}_{2}^{T}$. In terms of our language, the 2-cocycle $\omega_{2}$ and 1-cocycle $s_{1}$ have nonzero values $\omega_{2}(T, T)=1$ and $s_{1}(T)=1$, respectively. They are nontrivial cocycles in $H^{2}\left(\mathbb{Z}_{2}^{T}, \mathbb{Z}_{2}\right)=\mathbb{Z}_{2}$ and $H^{1}\left(\mathbb{Z}_{2}^{T}, \mathbb{Z}_{2}\right)=\mathbb{Z}_{2}$, respectively. By choosing different $\omega_{2}$ and $s_{1}$, we have three other fermionic symmetry groups $G_{f}: \mathbb{Z}_{2}^{f} \times \mathbb{Z}_{2}$ (trivial $\omega_{2}$ and trivial $s_{1}$ ), $\mathbb{Z}_{4}^{f}=\mathbb{Z}_{2}^{f} \times_{\omega_{2}} \mathbb{Z}_{2}$ (nontrivial $\omega_{2}$ and trivial $s_{1}$ ), and $\mathbb{Z}_{2}^{f} \times \mathbb{Z}_{2}^{T}$ (trivial $\omega_{2}$ and nontrivial $s_{1}$ ). We calculate the classifications of FSPT phases with these four fermionic symmetry groups in Appendix E 2.

\section{Summary of main results}

\section{Summary of data and equations}

As discussed above, to specify the total symmetry group $G_{f}$ of a fermionic system, we have a 1-cocycle $s_{1} \in H^{1}\left(G_{b}, \mathbb{Z}_{2}\right)$ which is related to time-reversal symmetry and a 2-cocycle $\omega_{2} \in H^{2}\left(G_{b}, \mathbb{Z}_{2}\right)$ which tells us how $G_{b}$ is extended by $\mathbb{Z}_{2}^{f}$. They satisfy the ( $\left.\bmod 2\right)$ cocycle equations:

$$
\begin{gathered}
d s_{1}=0, \\
d \omega_{2}=0 .
\end{gathered}
$$


TABLE I. Layers of classification data. The cochains $n_{d} \in C^{d}\left(G_{b}, \mathbb{Z}_{2}\right), n_{d-1} \in C^{d-1}\left(G_{b}, \mathbb{Z}_{2}\right)$, and $n_{d-2} \in$ $C^{d-2}\left(G_{b}, \mathbb{Z}_{T}\right)$ describe the decorations of 0D complex fermions, 1D Kitaev chains, and 2D $p+i p$ superconductors (SCs) in the $d$-spatial dimension model, respectively. And $\nu_{d+1} \in C^{d+1}\left[G_{b}, U(1)_{T}\right]$ is the bosonic $U(1)$ phase factor in the wave function, which is related to the group cohomology classification of BSPT phases in $d$-spatial dimension. There is also $n_{0}$ data in each dimension, if we want to classify fermionic invertible topological orders. We omit these $n_{0}$ states in this paper, for they do not need any $G_{b}$ symmetry protection. But they are important in the trivialization group which will be discussed below.

\begin{tabular}{|c|c|c|c|c|c|c|}
\hline Dataldim & & $1 \mathrm{D}$ & & $2 \mathrm{D}$ & & $3 \mathrm{D}$ \\
\hline$C^{1}\left(G_{b}, \cdot\right)$ & $n_{1}$ & Complex fermion & $n_{1}$ & Kitaev chain & $n_{1}$ & $p+i p$ superconductor \\
\hline$C^{2}\left(G_{b}, \cdot\right)$ & $\nu_{2}$ & Phase factor & $n_{2}$ & Complex fermion & $n_{2}$ & Kitaev chain \\
\hline$C^{3}\left(G_{b}, \cdot\right)$ & $\cdots$ & $\cdots$ & $\nu_{3}$ & Phase factor & $n_{3}$ & Complex fermion \\
\hline$C^{4}\left(G_{b}, \cdot\right)$ & $\cdots$ & $\cdots$ & $\cdots$ & $\cdots$ & $\nu_{4}$ & Phase factor \\
\hline
\end{tabular}

Given the input information of the total symmetry group $G_{f}$ (i.e., $G_{b}$ with $s_{1}$ and $\omega_{2}$ ), we summarize the classification data, symmetry conditions, consistency equations, and extra coboundary (states trivialized by ASPT state in one lower dimensions) for FSPT states in different physical dimensions in Eqs. (8)-(19) (see also Table I for the classification data and Table II for the physical meanings of the consistency equations).

We note that the cochains $n_{d} \in C^{d}\left(G_{b}, \mathbb{Z}_{2}\right), n_{d-1} \in$ $C^{d-1}\left(G_{b}, \mathbb{Z}_{2}\right)$, and $n_{d-2} \in C^{d-2}\left(G_{b}, \mathbb{Z}_{T}\right)$ describe the decorations of $0 \mathrm{D}$ complex fermions, 1D Kitaev chains, and 2D $p+i p$ superconductors (SCs) in the $d$-spatial dimension model, respectively. In $1 \mathrm{D}$, it is possible only to decorate a complex fermion onto the $G_{b}$ symmetry domain wall, and the constraint $d n_{1}=0$ is nothing but the fermion parity conservation requirement for a valid FSLU transformation. In 2D, it is possible to decorate both a Majorana chain onto the $G_{b}$ symmetry domain wall and a complex fermion onto the intersection point of $G_{b}$ symmetry domain walls. In order to construct FSPT states, we must decorate a closed Majorana chain onto the $G_{b}$ symmetry domain wall, and this constraint implies $d n_{1}=0$. Again, fermion parity conservation of FSLU transformation requires that $d n_{2}=\left(\omega_{2}+s_{1} \smile n_{1}\right) \smile n_{1}$. In $3 \mathrm{D}$, it is even possible to decorate a $2 \mathrm{D} p+i p$ SC state onto the $G_{b}$ symmetry domain wall if $G_{b}$ contains antiunitary symmetry. However, in order to construct such FSPT states, we must require that there is no chiral Majorana mode on the intersection lines of $G_{b}$ symmetry domain walls. Furthermore, $d n_{2}=$ $\left(\omega_{2}+s_{1} \smile n_{1}\right) \smile n_{1}$ corresponds to the absence of a free
Majorana fermion on the intersection points of $G_{b}$ symmetry domain walls, and $d n_{3}=\left(\omega_{2}+n_{2}\right) \smile n_{2}+s_{1} \smile$ $\left(n_{2} \smile{ }_{1} n_{2}\right)$ again corresponds to fermion parity conservation of FSLU transformation. Finally, the bosonic $U(1)-$ valued phase factor $\nu_{d+1} \in C^{d+1}\left[G_{b}, U(1)_{T}\right]$ must satisfy the so-called twisted cocycle condition $d \nu_{i}=\mathcal{O}_{i+1}$, which is generated by fixed-point conditions of FSPT wave functions. We note that the bosonic layer data $\nu_{d+1}$ without a superscript always mean the inhomogeneous cochain in the twisted cocycle equation. The homogeneous cochain is obtained by a symmetry action and may have additional sign factors. There is also a symmetry action on the first term of the coboundary definition in $d \nu_{d+1}$. Because timereversal symmetry has nontrivial actions on both $\mathbb{Z}_{T}$ and $U(1)_{T}$, there is an exponent $1-2 s_{1}\left(g_{0}\right)= \pm 1$ for the first term of $d \nu_{d+1}$.

Based on the above decoration construction, we can obtain the FSPT classifications by solving the consistency equations layer by layer as shown in Table II. The solutions of these equations can be used to construct FSPT states. And the final classifications are obtained from these data by quotient some subgroups. We note that $B^{i}$ are the coboundary subgroups defined for the corresponding cochain groups $C^{i}$ in the usual sense. The trivialization subgroups $\Gamma^{i}$ of the classification data correspond to the states that are trivialized by boundary ASPT states. In $d$ spatial dimensions, the $U(1)$ factor $\nu_{d+1}$ in $\Gamma^{d+1}$ corresponds to a BSPT state trivialized by fermions [52]. The complex fermion decoration data $n_{d}$ in the next layer $\Gamma^{d}$ are trivialized by boundary ASPT states with Kitaev chains [66]. And the

TABLE II. Consistency equations and their physical meanings for each layers. The physical meanings of the twisted equations (of the form $d n_{i}=\mathcal{O}_{i+1}$ or $\left.d \nu_{i}=\mathcal{O}_{i+1}\right)$ are given in the last column of the table. The explicit expressions of $\mathcal{O}_{4}\left[n_{2}\right]$ and $\mathcal{O}_{5}\left[n_{3}\right]$ are given in Eqs. (136) and (220), respectively.

\begin{tabular}{|c|c|c|c|c|}
\hline Layersldim & $1 \mathrm{D}$ & $2 \mathrm{D}$ & $3 \mathrm{D}$ & Physical meanings \\
\hline$p+i p \mathrm{SC}$ & $\ldots$ & . & $d n_{1}=0$ & No chiral Majorana mode \\
\hline Kitaev chain & $\cdots$ & $d n_{1}=0$ & $d n_{2}=\left(\omega_{2}+s_{1} \smile n_{1}\right) \smile n_{1}$ & No free Majorana fermion \\
\hline Complex fermion & $d n_{1}=0$ & $d n_{2}=\left(\omega_{2}+s_{1} \smile n_{1}\right) \smile n_{1}$ & $d n_{3}=\left(\omega_{2}+n_{2}\right) \smile n_{2}+s_{1} \smile\left(n_{2} \smile{ }_{1} n_{2}\right)$ & Fermion parity conservation \\
\hline Phase factor & $d \nu_{2}=(-)^{\omega_{2} \smile n_{1}}$ & $d \nu_{3}=\mathcal{O}_{4}\left[n_{2}\right]$ & $d \nu_{4}=\mathcal{O}_{5}\left[n_{3}\right]$ & Twisted cocycle equation \\
\hline
\end{tabular}


Kitaev chain decoration data $n_{d-1}$ in $\Gamma^{d-1}$ are trivialized by boundary ASPT states with 2D $p+i p$ chiral superconductors.

A subtle trivialization subgroup is $\Gamma^{4}$, which trivializes some 3D BSPT states in $H^{4}\left[G_{b}, U(1)_{T}\right]$ [see Eq. (19)]. Depending on whether the corresponding 2D ASPT state has $p+i p$ superconductor components or not, $\Gamma^{4}$ can be divided into two parts: $\Gamma^{4}=\Gamma_{n_{0}=0}^{4} \cup \Gamma_{n_{0} \neq 0}^{4}$. The first one $\Gamma_{n_{0}=0}^{4}$ is related to the ASPT state with boundary Majorana chain $n_{1}$ and complex fermion $n_{2}$ decorations [see the last line of Eq. (19) for the expression]. In this subgroup, the 2D ASPT state satisfies $d n_{2}=\mathcal{O}_{3}\left[n_{1}\right]$ in Eq. (14), and the 3D BSPT with 4-cocycle $\mathcal{O}_{4}\left[n_{2}\right]$ in Eq. (136) becomes a trivial 3D FSPT state. The second part $\Gamma_{n_{0} \neq 0}^{4}$ is related to $n_{0} \neq 0$ layers of $p+i p$ superconductors as 2D ASPT states. By gauging fermion parity, one can derive a complicated expression for $\Gamma_{n_{0} \neq 0}^{4}$ [67]. To the best of our knowledge, so far there is no known example of $G_{f}$ corresponding to a nontrivial solution of $\Gamma_{n_{0} \neq 0}^{4}$. Therefore, it is possible that $\Gamma_{n_{0} \neq 0}^{4}$ is always trivial for realistic physical systems, and we study the full derivation of $\Gamma_{n_{0} \neq 0}^{4}$ elsewhere.

Our FSPT classification results in different spatial dimensions are summarized below.

$$
\begin{gathered}
\text { 1D: }\left(n_{1}, \nu_{2}\right) \cdot- \\
n_{1} \in H^{1}\left(G_{b}, \mathbb{Z}_{2}\right), \\
\nu_{2} \in C^{2}\left[G_{b}, U(1)_{T}\right] / B^{2}\left[G_{b}, U(1)_{T}\right] / \Gamma^{2} . \\
\begin{aligned}
& n_{1}\left(g g_{0}, g g_{1}\right)=n_{1}\left(g_{0}, g_{1}\right)=n_{1}\left(g_{0}^{-1} g_{1}\right), \\
& \nu_{2}(g, g a, g a b)=g_{\nu_{2}}(a, b) \\
&= \nu_{2}(a, b)^{1-2 s_{1}(g)} \cdot(-1)^{\left(\omega_{2} \smile n_{1}\right)(g, a, b) .} . \\
& d n_{1}=0, \\
& d \nu_{2}=(-1)^{\omega_{2} \smile n_{1}} . \\
& \Gamma^{2}=\left\{(-1)^{\omega_{2}} \in H^{2}\left[G_{b}, U(1)_{T}\right]\right\} .
\end{aligned}
\end{gathered}
$$

2D: $\left(n_{1}, n_{2}, \nu_{3}\right) \cdot-$

$n_{1} \in H^{1}\left(G_{b}, \mathbb{Z}_{2}\right)$,

$n_{2} \in C^{2}\left(G_{b}, \mathbb{Z}_{2}\right) / B^{2}\left(G_{b}, \mathbb{Z}_{2}\right) / \Gamma^{2}$,

$\nu_{3} \in C^{3}\left[G_{b}, U(1)_{T}\right] / B^{3}\left[G_{b}, U(1)_{T}\right] / \Gamma^{3}$.

3D: $\left(n_{1}, n_{2}, n_{3}, \nu_{4}\right)$.-

$$
\begin{gathered}
d n_{1}=0, \\
d n_{2}=\omega_{2} \smile n_{1}+s_{1} \smile n_{1} \smile n_{1}, \\
d \nu_{3}=\mathcal{O}_{4}\left[n_{2}\right] \quad[\text { see Eq. }(137)] . \\
\Gamma^{2}=\left\{\omega_{2} \in H^{2}\left(G_{b}, \mathbb{Z}_{2}\right)\right\}, \\
\Gamma^{3}=\left\{(-1)^{\omega_{2} \smile n_{1}} \in H^{3}\left[G_{b}, U(1)_{T}\right] \mid n_{1} \in H^{1}\left(G_{b}, \mathbb{Z}_{2}\right)\right\} .
\end{gathered}
$$$$
n_{1} \in H^{1}\left(G_{b}, \mathbb{Z}_{T}\right),
$$$$
n_{2} \in C^{2}\left(G_{b}, \mathbb{Z}_{2}\right) / B^{2}\left(G_{b}, \mathbb{Z}_{2}\right) / \Gamma^{2},
$$$$
n_{3} \in C^{3}\left(G_{b}, \mathbb{Z}_{2}\right) / B^{3}\left(G_{b}, \mathbb{Z}_{2}\right) / \Gamma^{3},
$$$$
\nu_{4} \in C^{4}\left[G_{b}, U(1)_{T}\right] / B^{4}\left[G_{b}, U(1)_{T}\right] / \Gamma^{4} \text {. }
$$$$
n_{1}(g, g a)={ }^{g} n_{1}(e, a)={ }^{g} n_{1}(a)=(-1)^{s_{1}(g)} n_{1}(a),
$$$$
n_{2}\left(g g_{0}, g g_{1}, g g_{2}\right)=n_{2}\left(g_{0}, g_{1}, g_{2}\right)=n_{2}\left(g_{0}^{-1} g_{1}, g_{1}^{-1} g_{2}\right),
$$$$
n_{3}\left(g g_{0}, g g_{1}, g g_{2}, g g_{3}\right)=n_{3}\left(g_{0}, g_{1}, g_{2}, g_{3}\right)=n_{3}\left(g_{0}^{-1} g_{1}, g_{1}^{-1} g_{2}, g_{2}^{-1} g_{3}\right),
$$$$
\left.\nu_{4}(g, g a, g a b, g a b c, \text { gabcd })={ }^{g} \nu_{4}(a, b, c, d)=\nu_{4}(a, b, c, d)^{1-2 s_{1}(g)} \cdot \mathcal{O}_{5}^{\text {symm }}(g, g a, g a b, \text { gabc, gabcd }) \quad \text { [see Eq. }(212)\right] .
$$ 


$$
\begin{aligned}
& d n_{1}=0, \\
& d n_{2}=\omega_{2} \smile n_{1}+s_{1} \smile n_{1} \smile n_{1}, \\
& d n_{3}=\omega_{2} \smile n_{2}+n_{2} \smile n_{2}+s_{1} \smile\left(n_{2} \smile_{1} n_{2}\right), \\
& d \nu_{4}=\mathcal{O}_{5}\left[n_{3}\right] \quad[\text { see Eq. }(221)] .
\end{aligned}
$$

$\Gamma^{2}=\left\{\omega_{2} \smile n_{0} \in H^{2}\left(G_{b}, \mathbb{Z}_{2}\right) \mid n_{0} \in H^{0}\left(G_{b}, \mathbb{Z}_{T}\right)\right\}$,

$\Gamma^{3}=\left\{\omega_{2} \smile n_{1}+s_{1} \smile n_{1} \smile n_{1}+\left(\omega_{2} \smile_{1} \omega_{2}\right)\left\lfloor n_{0} / 2\right\rfloor \in H^{3}\left(G_{b}, \mathbb{Z}_{2}\right) \mid n_{1} \in H^{1}\left(G_{b}, \mathbb{Z}_{2}\right), n_{0} \in H^{0}\left(G_{b}, \mathbb{Z}_{T}\right)\right\}$,

$\Gamma^{4}=\left\{\mathcal{O}_{4}\left[n_{2}\right] \quad[\right.$ see Eq.(137) $] \in H^{4}\left(G_{b}, U(1)_{T}\right) \mid n_{2}$ satisfying Eq.(120) for some $\left.n_{1} \in H^{1}\left(G_{b}, \mathbb{Z}_{2}\right)\right\} \cup \Gamma_{n_{0} \neq 0}^{4}$.

\section{Summary of classification examples}

Using the above data, we calculate the classifications for FSPT phases with several simple symmetry groups. They are summarized in Table III. Some of the derivations are given in Appendix E. In particular, we calculate the classifications for 2D FSPT phases with arbitrary unitary finite Abelian group $G_{f}$ in Appendix E 1. Our results are exactly the same as that in Ref. [13], which uses a totally different approach by investigating the braiding statistics of the gauge flux. The calculations for 3D FSPT phases with arbitrary unitary finite Abelian group $G_{f}$ are given in Ref. [68]. The results are also consistent with 3D loop braiding statistics approaches. We calculate the classifications of FSPT phases for the four fermionic symmetry groups with $G_{b} \cong \mathbb{Z}_{2}$ in Appendix E2. They are also consistent with previously known results. As an example of non-Abelian $G_{f}$, we calculate the FSPT phases with

\begin{tabular}{|c|c|c|c|}
\hline$G_{f} \backslash \operatorname{dim}$ & 1 & 2 & 3 \\
\hline $\mathbb{Z}_{2}^{f} \times \mathbb{Z}_{2}$ & $\mathbb{Z}_{2}$ & $\mathbb{Z}_{8}$ & $\mathbb{Z}_{1}$ \\
\hline $\mathbb{Z}_{2}^{f} \times \mathbb{Z}_{2 k+1}$ & $\mathbb{Z}_{1}$ & $\mathbb{Z}_{2 k+1}$ & $\mathbb{Z}_{1}$ \\
\hline $\mathbb{Z}_{2}^{f} \times \mathbb{Z}_{2 k}$ & $\mathbb{Z}_{2}$ & $\begin{cases}\mathbb{Z}_{4 k} \times \mathbb{Z}_{2}, & k \text { even } \\
\mathbb{Z}_{8 k}, & k \text { odd }\end{cases}$ & $\mathbb{Z}_{1}$ \\
\hline $\mathbb{Z}_{2}^{f} \times \mathbb{Z}_{2} \times \mathbb{Z}_{2}$ & $\mathbb{Z}_{2}^{3}$ & $\mathbb{Z}_{8}^{2} \times \mathbb{Z}_{4}$ & $\mathbb{Z}_{2}^{2}$ \\
\hline $\mathbb{Z}_{2}^{f} \times \mathbb{Z}_{2} \times \mathbb{Z}_{4}$ & $\mathbb{Z}_{2}^{3}$ & $\mathbb{Z}_{8}^{2} \times \mathbb{Z}_{2}^{3}$ & $\mathbb{Z}_{4} \times \mathbb{Z}_{2}$ \\
\hline $\mathbb{Z}_{2}^{f} \times \mathbb{Z}_{2} \times \mathbb{Z}_{8}$ & $\mathbb{Z}_{2}^{3}$ & $\mathbb{Z}_{16} \times \mathbb{Z}_{8} \times \mathbb{Z}_{2}^{3}$ & $\mathbb{Z}_{8} \times \mathbb{Z}_{2}$ \\
\hline $\mathbb{Z}_{2}^{f} \times \mathbb{Z}_{4} \times \mathbb{Z}_{4}$ & $\mathbb{Z}_{2}^{2} \times \mathbb{Z}_{4}$ & $\mathbb{Z}_{8}^{2} \times \mathbb{Z}_{4} \times \mathbb{Z}_{2}^{3}$ & $\mathbb{Z}_{4}^{2} \times \mathbb{Z}_{2}$ \\
\hline $\mathbb{Z}_{2}^{f} \times \mathbb{Z}_{4} \times \mathbb{Z}_{8}$ & $\mathbb{Z}_{2}^{2} \times \mathbb{Z}_{4}$ & $\mathbb{Z}_{16} \times \mathbb{Z}_{8} \times \mathbb{Z}_{4} \times \mathbb{Z}_{2}^{3}$ & $\mathbb{Z}_{8} \times \mathbb{Z}_{4} \times \mathbb{Z}_{2}$ \\
\hline $\mathbb{Z}_{2}^{f} \times \mathbb{Z}_{2} \times \mathbb{Z}_{2} \times \mathbb{Z}_{2}$ & $\mathbb{Z}_{2}^{4}$ & $\mathbb{Z}_{8}^{3} \times \mathbb{Z}_{4}^{3} \times \mathbb{Z}_{2}$ & $\mathbb{Z}_{2}^{8}$ \\
\hline $\mathbb{Z}_{2}^{f} \times \mathbb{Z}_{2} \times \mathbb{Z}_{2} \times \mathbb{Z}_{4}$ & $\mathbb{Z}_{2}^{4}$ & $\mathbb{Z}_{8}^{3} \times \mathbb{Z}_{4} \times \mathbb{Z}_{2}^{6}$ & $\mathbb{Z}_{4}^{3} \times \mathbb{Z}_{2}^{5}$ \\
\hline $\mathbb{Z}_{2}^{f} \times \mathbb{Z}_{2} \times \mathbb{Z}_{4} \times \mathbb{Z}_{4}$ & $\mathbb{Z}_{2}^{4}$ & $\mathbb{Z}_{8}^{3} \times \mathbb{Z}_{4} \times \mathbb{Z}_{2}^{8}$ & $\mathbb{Z}_{4}^{4} \times \mathbb{Z}_{2}^{6}$ \\
\hline $\mathbb{Z}_{2 k}^{f}\left(k=2^{n} \geq 2\right)$ & $\mathbb{Z}_{1}$ & $\mathbb{Z}_{k / 2}$ & $\mathbb{Z}_{1}$ \\
\hline $\mathbb{Z}_{4}^{f} \times \mathbb{Z}_{2}$ & $\mathbb{Z}_{2}$ & $\mathbb{Z}_{4}$ & $\mathbb{Z}_{2}$ \\
\hline $\mathbb{Z}_{4}^{f} \times \mathbb{Z}_{4}$ & $\mathbb{Z}_{4}$ & $\mathbb{Z}_{8} \times \mathbb{Z}_{2}$ & $\mathbb{Z}_{2}$ \\
\hline $\mathbb{Z}_{8}^{f} \times \mathbb{Z}_{2}$ & $\mathbb{Z}_{2}$ & $\mathbb{Z}_{4} \times \mathbb{Z}_{2}$ & $\mathbb{Z}_{4}$ \\
\hline $\mathbb{Z}_{4}^{f} \times \mathbb{Z}_{2} \times \mathbb{Z}_{2}$ & $\mathbb{Z}_{2}^{3}$ & $\mathbb{Z}_{4}^{2} \times \mathbb{Z}_{2}^{2}$ & $\mathbb{Z}_{2}^{5}$ \\
\hline $\mathbb{Z}_{4}^{f} \times \mathbb{Z}_{2} \times \mathbb{Z}_{4}$ & $\mathbb{Z}_{4} \times \mathbb{Z}_{2}^{2}$ & $\mathbb{Z}_{8} \times \mathbb{Z}_{4} \times \mathbb{Z}_{2}^{3}$ & $\mathbb{Z}_{4} \times \mathbb{Z}_{2}^{4}$ \\
\hline $\mathbb{Z}_{4}^{f} \times \mathbb{Z}_{4} \times \mathbb{Z}_{4}$ & $\mathbb{Z}_{4}^{3}$ & $\mathbb{Z}_{8}^{2} \times \mathbb{Z}_{4}^{2} \times \mathbb{Z}_{2}^{2}$ & $\mathbb{Z}_{4}^{2} \times \mathbb{Z}_{2}^{4}$ \\
\hline $\mathbb{Z}_{2}^{f} \times \mathbb{Z}_{2}^{T}$ & $\mathbb{Z}_{4}$ & $\mathbb{Z}_{1}$ & $\mathbb{Z}_{1}$ \\
\hline $\mathbb{Z}_{4}^{T f}=\mathbb{Z}_{2}^{f} \times_{\omega_{2}} \mathbb{Z}_{2}^{T}$ & $\mathbb{Z}_{2}$ & $\mathbb{Z}_{2}$ & $\mathbb{Z}_{16}$ \\
\hline$Q_{8}^{f}=\mathbb{Z}_{2}^{f} \times_{\omega_{2}}\left(\mathbb{Z}_{2} \times \mathbb{Z}_{2}\right)$ & $\mathbb{Z}_{1}$ & $\mathbb{Z}_{2}$ & $\mathbb{Z}_{1}$ \\
\hline
\end{tabular}
quaternion group symmetry $G_{f}=Q_{8}^{f}$ in Appendix E 3 .

TABLE III. Classifications of FSPT phases with some simple fermionic symmetry group $G_{f}$ in different spatial dimensions. Invertible topological orders protected by $\mathbb{Z}_{2}^{f}$ only are not included in this table. 


\section{Organization of the paper}

The rest of the paper is organized as follows. In Sec. II, we review the key concept of SLU transformations. Using this approach, we show the classifications of BSPT phases in various dimensions. In Sec. III, we summarize the procedures of constructing FSPT states. The definition of FSLU transformations is given in Sec. III A. All layers of degrees of freedom and their symmetry transformation rules are summarized in Sec. III B. In Sec. III C, we discuss briefly the two essential requirements of the FSLU transformations: the coherence equations and the symmetry conditions. Using the outlined procedure, the details of the classifications of 1D, 2D, and 3D FSPT phases are given in Secs. IV, V, and VI, respectively. In each dimension, we first give the symmetric decoration procedures. Then, the $F$ move (FSLU transformation) and its coherence equation are given explicitly. As the final step in classifying FSPT phases in each dimension, we discuss some new coboundaries associated with ASPT states in one lower dimension. We summarize this work in Sec. VII.

In Appendix A, we show the classification of the simplest 0D FSPT phases. In Appendix B, we list all possible $2 \mathrm{D}$ and $3 \mathrm{D}$ moves that admit a branching structure. In Appendix C, the (local) Kasteleyn orientations for 2D and 3D lattices are discussed briefly. In Appendix D, we discuss the Bockstein homomorphism mapping a $\mathbb{Z}_{2}^{-}$ valued cocycle to a $\mathbb{Z}_{T}$-valued cocycle. It is useful in checking whether the obstruction function $(-1)^{f_{k}}$, where $f_{k}$ is a $\mathbb{Z}_{2}$-valued cocycle, is a $U(1)_{T}$-valued coboundary or not. The detail calculations of FSPT phases for some simple groups are given in Appendix E. Some of the results are already summarized in Table III.

\section{SLU TRANSFORMATION AND CLASSIFICATION OF BSPT PHASES}

\section{A. SLU transformation and BSPT phases}

From the definition of SPT states, it is easy to see that (in the absence of global symmetry)

$$
\left.|\mathrm{SPT}\rangle=U_{\text {circ }}^{M} \mid \text { trivial }\right\rangle .
$$

Namely, an SPT state can be connected to a trivial state (e.g., a product state) via LU transformation (in the absence of global symmetry). Clearly, Eq. (20) implies that the support space [69] of any SPT state in a region must be one dimensional. This result is simply because a trivial state (e.g., a product state) has a one-dimensional support space, and any SPT state will become a product state via a proper local basis change (induced by a LU transformation).

In the presence of global symmetry, we can further introduce the notion of SLU transformations classifying SPT phases in interacting bosonic systems. By SLU transformation, we mean the corresponding piecewise LU operator is invariant under symmetry $G$. More precisely, we have $U_{p w l}=\prod_{i} e^{-i H_{b}\left(g_{i 0}, g_{i 1}, g_{i 2}, \ldots\right)} \equiv \prod_{i} U\left(g_{i 0}, g_{i 1}, g_{i 2}, \ldots\right) \quad$ and $U\left(g g_{i 0}, g g_{i 2}, g g_{i 3}, \ldots\right)=U\left(g_{i 0}, g_{i 1}, g_{i 2}, \ldots\right)$ for any $g \in G$. (We note that here we choose the group element basis $g_{i 0}, g_{i 1}, g_{i 2}, \ldots$ to represent bosonic symmetric unitary operator acting on a region labeled by $i$.) However, we need to enforce the SLU transformations to be one dimensional (when acting on the support space $\rho_{A}$ for any region $A$ ), and we call them invertible SLU transformations. Thus, we claim that SPT phases in interacting bosonic systems can be classified by an equivalence class of invertible SLU transformations.

SPT phases are also referred to as invertible (nonchiral) topological phases. It turns out that the novel concept of invertible SLU transformation even allows us to construct very general fixed-point SPT states. All of these fixed-point wave functions admit exactly solvable parent Hamiltonians consisting of commuting projectors on an arbitrary triangulation with an arbitrary branching structure.

\section{B. Fixed-point wave function and classification for BSPT phases in 1D}

As a warm-up, let us begin with fixed-point wave function in 1D and use SLU transformation to derive the well-known classification results of 1D BSPT phases. Without the loss of generality, here we assume that every (locally ordered) vertex $i$ of the 1D lattice has bosonic degrees of freedom labeled by a group element $g_{i} \in G$.

Our 1D fixed-point state is a superposition of those basis states with all possible 1D graph with a branching structure (denoted by the arrow from left to right):

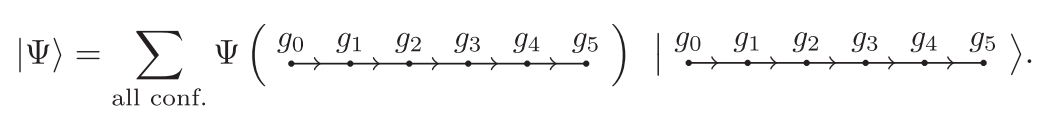

In the following, we derive the rules of wave function renormalization generated by SLU transformations for the above wave function and show how to construct all BSPT states in 1D. To obtain a fixed-point wave function, we need to understand the changes of the wave function under renormalization. In 1D, renormalization can be understood as removing some degrees of freedom by reducing the number of vertices. The basic renormalization process is known as the (2-1) Pachner move of triangulation of 1D manifold.

To be more precise, the (2-1) move is an SLU transformation between two different $1 \mathrm{D}$ graphs: 
$\Psi\left(\stackrel{g_{0} \longrightarrow \stackrel{g}{\bullet} \longrightarrow g_{2}}{\bullet}\right)=\frac{1}{|G|^{1 / 2}} \nu_{2}\left(g_{0}, g_{1}, g_{2}\right) \Psi\left(\stackrel{g_{0} \longrightarrow}{\longrightarrow} g_{2}\right)$,

We note that the $|G|$ is the order of the group $G$, and we introduce the normalization factor $1 /|G|^{1 / 2}$ in the above expression due to the change of vertex number. Here, $\nu_{2}\left(g_{0}, g_{1}, g_{2}\right)$ is a $U(1)$-valued function with variables $g_{i} \in G$. Since we are constructing a symmetric state, $\nu_{2}$ should be symmetric under the action of $G$ with $\nu_{2}\left(g g_{0}, g g_{1}, g g_{2}\right)=\nu_{2}\left(g_{0}, g_{1}, g_{2}\right) \quad$ [we note that $\nu_{2}\left(g g_{0}, g g_{1}, g g_{2}\right)=\nu_{2}^{*}\left(g_{0}, g_{1}, g_{2}\right)$ if $g$ is antiunitary].

Since we are constructing a fixed-point wave function, it should be invariant under renormalization. For instance, we can use two different sequences of the above (2-1) moves Eq. (22) to connect a fixed initial state and a fixed final state. Different approaches should give rise to the same wave function. These constraints give us the consistent equations for $\nu_{2}$.

The simplest example is the following two paths between two fixed states:

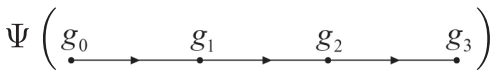

$$
\begin{aligned}
& =\frac{1}{|G|^{1 / 2}} \nu_{2}\left(g_{1}, g_{2}, g_{3}\right) \Psi\left(\stackrel{g_{0} \longrightarrow g_{1} \longrightarrow g_{3}}{\bullet} \longrightarrow\right. \\
& =\frac{1}{|G|} \nu_{2}\left(g_{1}, g_{2}, g_{3}\right) \nu_{2}\left(g_{0}, g_{1}, g_{3}\right) \Psi\left(\stackrel{g_{0} \longrightarrow}{g_{3}}\right) \text {, }
\end{aligned}
$$

$$
\begin{aligned}
& \Psi\left(\stackrel{g_{0}}{\bullet} \stackrel{g_{1}}{\bullet} \stackrel{g_{2}}{\bullet}, g_{3}\right) \\
& =\frac{1}{|G|^{1 / 2}} \nu_{2}\left(g_{0}, g_{1}, g_{2}\right) \Psi\left(\stackrel{g_{0} \longrightarrow g_{2} \longrightarrow g_{3}}{\bullet} \longrightarrow\right. \\
& =\frac{1}{|G|} \nu_{2}\left(g_{0}, g_{1}, g_{2}\right) \nu_{2}\left(g_{0}, g_{2}, g_{3}\right) \Psi\left(\stackrel{g_{0} \longrightarrow}{g_{3}}\right) \text {. }
\end{aligned}
$$

The constraint is that the products of $F$ moves for the above two processes equal each other:

$\nu_{2}\left(g_{0}, g_{1}, g_{3}\right) \nu_{2}\left(g_{1}, g_{2}, g_{3}\right)=\nu_{2}\left(g_{0}, g_{2}, g_{3}\right) \nu_{2}\left(g_{0}, g_{1}, g_{2}\right)$.

The above equation implies

$d \nu_{2}\left(g_{0}, g_{1}, g_{2}, g_{3}\right)=\frac{\nu_{2}\left(g_{1}, g_{2}, g_{3}\right) \nu_{2}\left(g_{0}, g_{1}, g_{3}\right)}{\nu_{2}\left(g_{0}, g_{2}, g_{3}\right) \nu_{2}\left(g_{0}, g_{1}, g_{2}\right)}=1$,

which is exactly the same as the cocycle equation of group cohomology theory, and it means $\nu_{2}$ should be a $U(1)_{T}$-valued 2-cocycle.

Using an SLU transformation, we can further redefine the basis state $\left|\left\{g_{l}\right\}\right\rangle$ as

$$
\left|\left\{g_{l}\right\}\right\rangle^{\prime}=U_{\mu_{1}, m_{0}}\left|\left\{g_{l}\right\}\right\rangle=\prod_{\langle i j\rangle} \mu_{1}\left(g_{i}, g_{j}\right)\left|\left\{g_{l}\right\}\right\rangle \text {. }
$$

In the new basis, one finds that the phase factor in Eq. (22) becomes

$\nu_{2}^{\prime}\left(g_{0}, g_{1}, g_{2}\right) \equiv \nu_{2}\left(g_{0}, g_{1}, g_{2}\right) \frac{\mu_{1}\left(g_{1}, g_{2}\right) \mu_{1}\left(g_{0}, g_{1}\right)}{\mu_{1}\left(g_{0}, g_{2}\right)}$.

Since our gapped phases are defined by SLU transformations, $\nu_{2}^{\prime}$ and $\nu_{2}$ belong to the same phase. In general, the elements $\nu_{2}$ in the same group cohomology class in $H^{2}\left[G, U(1)_{T}\right]$ correspond to the same 1D BSPT phase.

SLU transformations not only give rise to the local rules of constructing fixed-point wave functions, but also give rise to commuting-projector parent Hamiltonian for these fixed-point wave functions. In $1 \mathrm{D}$, the parent Hamiltonian can be expressed as $H=-\sum_{i} H_{i}$, where the matrix element of $H_{i}$ are defined as

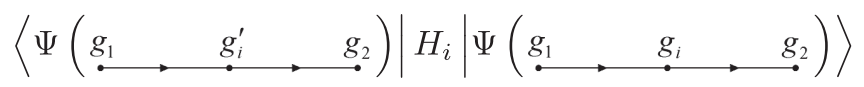

which acts only on the states on site $i$ and its neighboring sites. However, $H_{i}$ does not alter the states on neighboring sites of $i$.

The above amplitude can be computed by SLU transformations by considering the following moves for a threesite patch:

$$
\begin{aligned}
\Psi & \left(\stackrel{g_{1} \longrightarrow}{\bullet} g_{i}, g_{2}\right) \\
& =\frac{1}{|G|^{1 / 2}} \nu_{2}\left(g_{1}, g_{i}, g_{2}\right) \Psi\left(\stackrel{g_{1}, g_{2}}{\bullet}\right. \\
& =\frac{1}{|G|} \sum_{g_{i}^{\prime}} \frac{\nu_{2}\left(g_{1}, g_{i}, g_{2}\right)}{\nu_{2}\left(g_{1}, g_{i}^{\prime}, g_{2}\right)} \Psi\left(\stackrel{g_{1}, g_{i}^{\prime}, g_{2}}{\bullet}\right),
\end{aligned}
$$

which implies that

$$
\begin{aligned}
H_{i}^{g_{i}^{\prime} g_{i}}\left\langle g_{i}^{\prime}, g_{1} g_{2}\left|H_{i}\right| g_{i}, g_{1} g_{2}\right\rangle & =\frac{1}{|G|} \frac{\nu_{2}\left(g_{1}, g_{i}, g_{2}\right)}{\nu_{2}\left(g_{1}, g_{i}^{\prime}, g_{2}\right)} \\
& =\frac{1}{|G|} \frac{\nu_{2}\left(g_{1}, g_{i}, g_{i}^{\prime}\right)}{\nu_{2}\left(g_{i}, g_{i}^{\prime}, g_{2}\right)},
\end{aligned}
$$

where we use the 2-cocycle condition of $\nu_{2}$ in the last step. Clearly, $H_{i}$ is an Hermitian projector that satisfies $H_{i}^{\dagger}=H_{i}$ and $H_{i}^{2}=H_{i}$. Moreover, the 2-cocycle condition Eq. (25) further guarantees that all $H_{i}$ on different sites commute with each other. As an operator, we can also just define the action of $H_{i}$ on site $i$ and its neighboring sites 1,2 as

$$
H_{i}\left|g_{i}, g_{1} g_{2}\right\rangle=\frac{1}{|G|} \sum_{g_{i}^{\prime}} \frac{\nu_{2}\left(g_{1}, g_{i}, g_{i}^{\prime}\right)}{\nu_{2}\left(g_{i}, g_{i}^{\prime}, g_{2}\right)}\left|g_{i}^{\prime}, g_{1} g_{2}\right\rangle \text {. }
$$




\section{Fixed-point wave function and classification for BSPT phases in 2D}

The fixed-point wave functions for BSPT phases in 2D are similar to the $1 \mathrm{D}$ case. We can again use the group element basis to construct the local Hilbert space on each vertex of arbitrary triangulation:

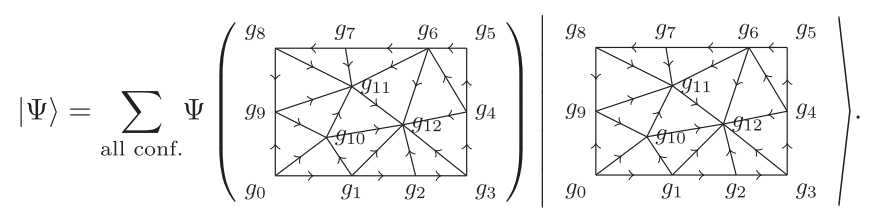

We assume that the triangulation admits a branching structure that can be labeled by a set of local arrows on all links (edges) with no oriented loop for any triangle. Mathematically, the branching structure can be regarded as a discrete version of a $\operatorname{spin}^{c}$ structure and can be consistently defined on arbitrary triangulation of orientable manifolds. The basic renormalization process is known as the (2-2) and (2-0)/(0-2) Pachner move of triangulation of 2D manifold. Moreover, according to the definition of BSPT phases, we also require that the support space of SLU transformations be one dimensional, such that it can adiabatically connect to a product state in the absence of global symmetry. Below, we discuss physically consistent conditions for those SLU transformations generating fixedpoint wave functions.

An example of the (2-2) move [now we call it the standard (2-2) move, which is the analogy of the $F$ move in a unitary fusion category theory] is presented as follows:

$$
\Psi(Y_{g_{1}}^{g_{3}} \underbrace{g_{2}}_{g_{2}})=\nu_{3}\left(g_{0}, g_{1}, g_{2}, g_{3}\right) \Psi\left(Z_{g_{1}}^{g_{3}}\right) .
$$

Here, $\nu_{3}\left(g_{0}, g_{1}, g_{2}, g_{3}\right)$ is a $U(1)$-valued 3-cochain that is symmetric under $g$ action $\nu_{3}\left(g g_{0}, g g_{1}, g g_{2}, g g_{3}\right)=\nu_{3}\left(g_{0}\right.$, $\left.g_{1}, g_{2}, g_{3}\right)$ [again, $\nu_{3}\left(g g_{0}, g g_{1}, g g_{2}, g g_{3}\right)=\nu_{3}^{*}\left(g_{0}, g_{1}, g_{2}, g_{3}\right)$ if $g$ is antiunitary].

Apart from the (2-2) move, there is another (2-0) move that can change the total number of vertices for triangulations:

$$
\Psi\left(g_{1}\langle\overbrace{g_{0}}^{g_{2}})=\frac{1}{|G|^{1 / 2}} \Psi\left(\left.\right|_{g_{1}} ^{g_{2}}\right) .\right.
$$

We also add a normalization factor $|G|^{-1 / 2}$ in front of the (2-0) move operator, for the vertex number is reduced by one from the left state to the right state [70].

It is easy to check that the other (2-2) moves with different branching structure, e.g., the analogy of the $H$ move, can always be derived by the standard (2-2) move and $(2-0) /(0-2)$ move. Consider the SLU transformation for the following patch:

$$
\begin{aligned}
& \Psi(g_{2} \overbrace{g_{0}}^{g_{3}} \gamma_{g_{1}}^{g_{2}}) \\
& =\nu_{3}\left(g_{0}, g_{1}, g_{2}, g_{3}\right) \Psi\left(g_{2}\langle\overbrace{g_{0}}^{g_{3}} g_{g_{1}})\right. \\
& =\frac{1}{|G|^{1 / 2}} \nu_{3}\left(g_{0}, g_{1}, g_{2}, g_{3}\right) \Psi\left(\begin{array}{c}
g_{3} \\
g_{g_{2}} \\
g_{g_{1}}
\end{array}\right) \\
& =\nu_{3}\left(g_{0}, g_{1}, g_{2}, g_{3}\right) \Psi(\underbrace{}_{g_{0}} \underbrace{g_{3}}_{g_{1}}) \text {, }
\end{aligned}
$$

The above deformation scheme implies that

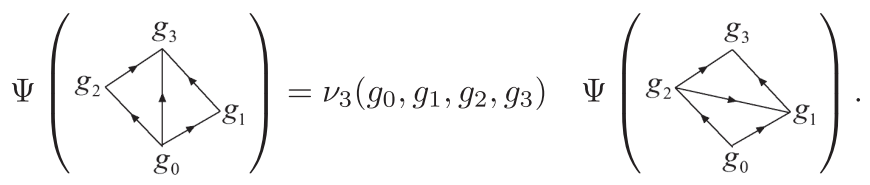

The remaining (2-2) moves are the analogies of the dual $\mathrm{F}$ move and dual $\mathrm{H}$ move; they can also be derived from the basis (2-2) move and (2-0)/(0-2) move. For example, let us assume

$$
\Psi(g_{g_{1}}^{g_{2}} \overbrace{g_{0}}^{g_{3}})=\bar{\nu}_{3}\left(g_{0}, g_{1}, g_{2}, g_{3}\right) \Psi\left(\stackrel{g_{2}}{g_{1}} \prod_{g_{0}}^{g_{3}}\right),
$$

where $\bar{\nu}_{3}\left(g_{0}, g_{1}, g_{2}, g_{3}\right)$ is another $U(1)$-valued function which is different from $\nu_{3}\left(g_{0}, g_{1}, g_{2}, g_{3}\right)$. Consider the SLU transformation on the following patch: 


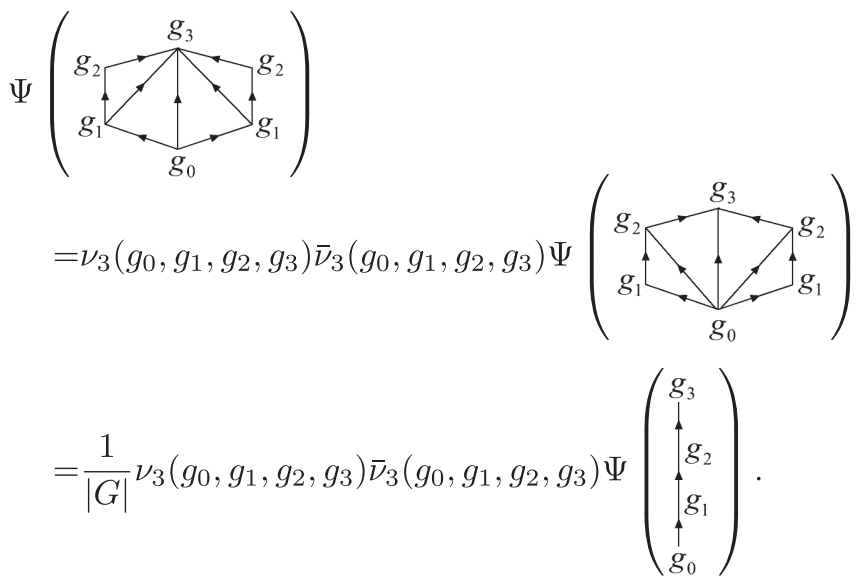

On the other hand, by applying the (2-0) move directly, we have

$$
\Psi(\underset{g_{0}}{g_{2}} \underbrace{g_{3}}_{g_{1}} g_{2})=\frac{1}{|G|} \Psi\left(\begin{array}{l}
g_{3} \\
\hat{f} \\
g_{2} \\
g_{1} \\
g_{0}
\end{array}\right) .
$$

The above two reduction schemes should agree, which implies that $\bar{\nu}_{3}\left(g_{0}, g_{1}, g_{2}, g_{3}\right) \nu_{3}\left(g_{0}, g_{1}, g_{2}, g_{3}\right)=1$ or $\bar{\nu}_{3}\left(g_{0}, g_{1}, g_{2}, g_{3}\right)=\nu_{3}^{-1}\left(g_{0}, g_{1}, g_{2}, g_{3}\right)$.

Moreover, the combination of the (2-2) move and (2-0) move further allows us to define a new set of renormalization moves which reduces the number of vertices, namely, the (3-1) move. For example, consider the SLU transformation for the following patch:

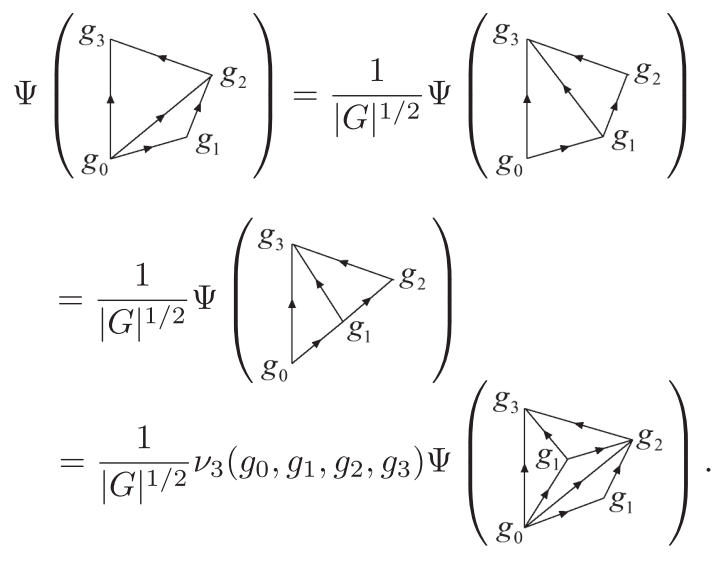

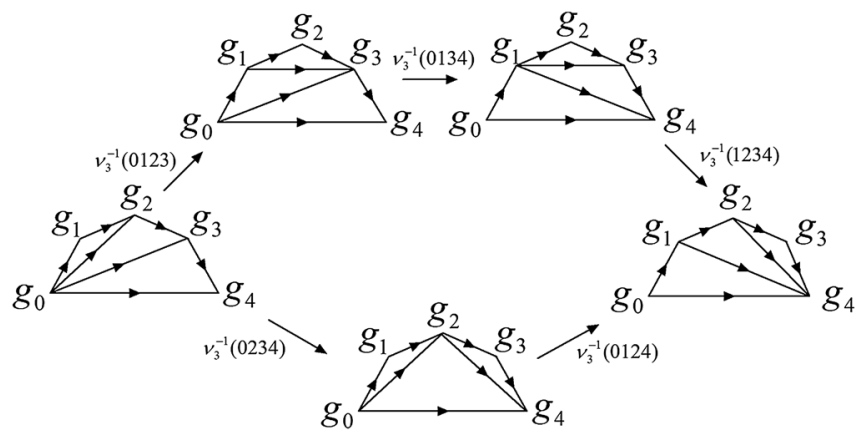

FIG. 1. The self-consistent equation on a big patch. The branching structure on this patch is induced by a local direction from left to right. Mathematically, it is known as the 3-cocycle equation.

The above deformation scheme implies that

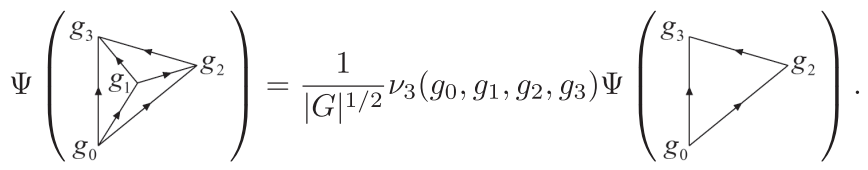

In Fig. 16 of Appendix B, we list all possible (2-2) and (3-1) moves that are consistent with a branching structure. In the above, we discuss the SLU moves. The most important one is the standard (2-2) move in Eq. (34). Similar to the 1D case, if we apply the (2-2) move for bigger patch as seen in Fig. 1, we can derive the consistent conditions for $\nu_{3}$ describing fixed-point wave functions:

$$
\begin{aligned}
& \left(d \nu_{3}\right)\left(g_{0}, g_{1}, g_{2}, g_{3}, g_{4}\right) \\
& \equiv \frac{\nu_{3}\left(g_{1}, g_{2}, g_{3}, g_{4}\right) \nu_{3}\left(g_{0}, g_{1}, g_{3}, g_{4}\right) \nu_{3}\left(g_{0}, g_{1}, g_{2}, g_{3}\right)}{\nu_{3}\left(g_{0}, g_{2}, g_{3}, g_{4}\right) \nu_{3}\left(g_{0}, g_{1}, g_{2}, g_{4}\right)}=1 .
\end{aligned}
$$

Mathematically, this equation is known as the 3-cocycle equation.

Similar to the 1D case, we can use SLU to redefine the basis state $\left|\left\{g_{l}\right\}\right\rangle$ as

$$
\left|\left\{g_{l}\right\}\right\rangle^{\prime}=U_{\mu_{2}}\left|\left\{g_{l}\right\}\right\rangle=\prod_{\langle i j k\rangle} \mu_{2}\left(g_{i}, g_{j}, g_{k}\right)^{s_{\langle i j k\rangle}}\left|\left\{g_{l}\right\}\right\rangle,
$$

where $s_{\langle i j k\rangle}= \pm 1$ denotes the orientation of the triangle $\langle i j k\rangle$. One finds that the phase factor in Eq. (34) becomes

$$
\begin{aligned}
& \nu_{3}^{\prime}\left(g_{0}, g_{1}, g_{2}, g_{3}\right) \\
& \quad=\nu_{3}\left(g_{0}, g_{1}, g_{2}, g_{3}\right) \frac{\mu_{2}\left(g_{1}, g_{2}, g_{3}\right) \mu_{2}\left(g_{0}, g_{1}, g_{3}\right)}{\mu_{2}\left(g_{0}, g_{2}, g_{3}\right) \mu_{2}\left(g_{0}, g_{1}, g_{2}\right)} .
\end{aligned}
$$


So the elements $\nu_{3}$ in the same group cohomology class in $H^{3}\left[G, U(1)_{T}\right]$ correspond to the same 2D BSPT phase.

Again, similar to the 1D case, the 2D SLU transformations can also be used to construct commutingprojector parent Hamiltonian for these fixed-point wave functions. The Hamiltonian is a summation of vertex terms as $H=-\sum_{*} H_{*}$. Again, the matrix element $H_{*}^{g_{*}, g_{*}}$ can be evaluated as a product of a sequence of moves that change the group element of vertex $*$ from $g_{*}$ to another $g_{*}^{\prime}$. For example, we can consider the following moves for a triangular lattice:

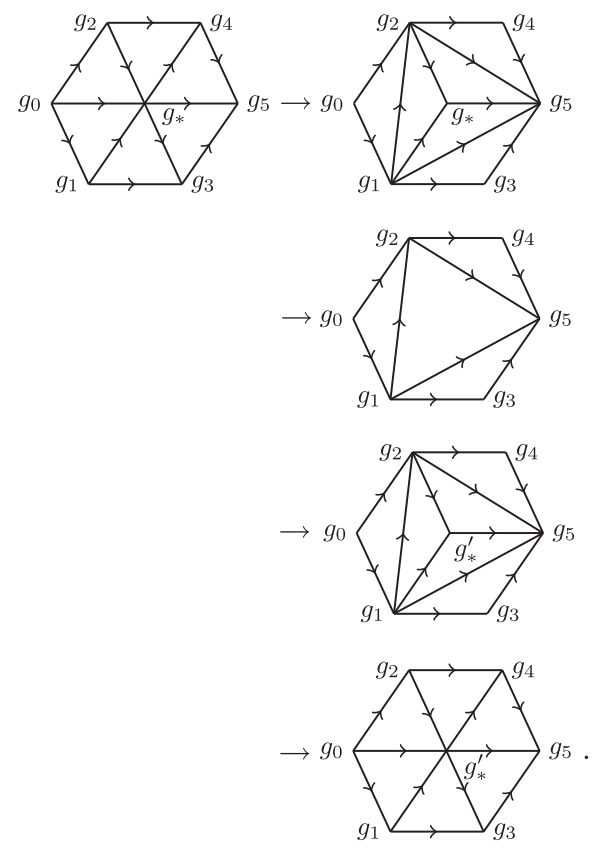

We shift the lattice a little, such that the branching structure is induced by a local direction from left to right. The first step of the above figures is a combination of three (2-2) moves. The second step is a (3-1) move that removes the vertex with group label $g_{*}$ at the center. The third step is a (1-3) move that creates a vertex with group label $g_{*}^{\prime}$ at the center. And the last step is a combination of three (2-2) moves that change the lattice to the original shape. Since our wave function is at the fixed point and $\nu_{3}$ satisfy the 3-cocycle condition Eq. (43), the terms $H_{*}$ for different vertices commute with each other. Clearly, $H_{*}$ is also a Hermitian projection operator satisfying $H_{*}^{\dagger}=H_{*}$ and $H_{*}^{2}=H_{*}$. Thus, the constructed Hamiltonian as a summation of $H_{*}$ terms is a commuting-projector parent Hamiltonian.

\section{Fixed-point wave function and classification for BSPT phases in 3D}

The fixed-point wave functions for BSPT phases in 3D are similar to the $1 \mathrm{D}$ and $2 \mathrm{D}$ cases. We can again use the group element basis to construct the local Hilbert space on each vertex of arbitrary triangulation. The basic renormalization process is known as the (2-3) and (2-0) Pachner move of triangulation of 3D manifold:

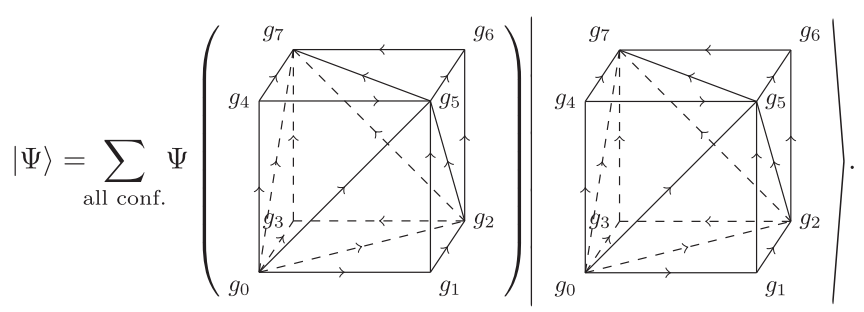

An example of the (2-3) move [now we call it the standard (2-3) move] is presented as follows:

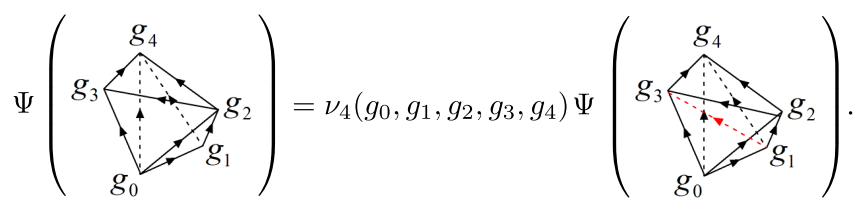

Here, $\nu_{4}\left(g_{0}, g_{1}, g_{2}, g_{3}, g_{4}\right)$ is a $U(1)$-valued 4-cochain that is symmetric under $g$ action $\nu_{4}\left(g g_{0}, g g_{1}, g g_{2}, g g_{3}, g g_{4}\right)=$ $\nu_{4}\left(g_{0}, g_{1}, g_{2}, g_{3}, g_{4}\right)\left[\nu_{4}\left(g g_{0}, g g_{1}, g g_{2}, g g_{3}, g g_{4}\right)=\nu_{4}^{*}\left(g_{0}, g_{1}\right.\right.$, $\left.g_{2}, g_{3}, g_{4}\right)$ if $g$ is antiunitary].

Again, apart from the (2-3) move, there are two (2-0) moves consisting with the branching structure that can change the total number of vertices for triangulations:

$$
\Psi\left(g_{g_{0}}^{g_{2}} g_{g_{1}}^{g_{3}}=\frac{1}{|G|^{1 / 2}} \Psi\left(g_{g_{0}}^{g_{3}} g_{2}\right)\right.
$$

and

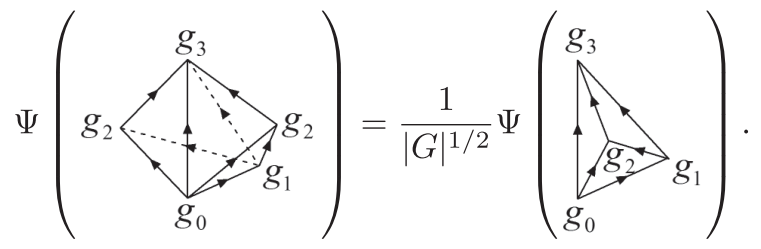

Again, we add a normalization factor $|G|^{-1 / 2}$ in front of the (2-0) move operator, for the vertex number is reduced by one from the left state to the right state [71].

It is easy to check that other (2-3) moves with different branching structure can always be generated by the standard (2-3) move and (2-0)/(0-2) move. Moreover, the combination of the (2-3) move and (2-0) move further allows us to define a new set of renormalization moves which reduces the number of vertices, namely, the (4-1) 
move. In Figs. 17 and 18 of Appendix B, we list all possible (2-3) and (4-1) moves that admit a branching structure.

In the above, we discuss the SLU moves. The most important one is the standard (2-3) move in
Eq. (48). Similar to the $1 \mathrm{D}$ and $2 \mathrm{D}$ cases, if we apply the (2-3) move for a bigger patch, we can derive the consistent conditions for $\nu_{4}$ describing fixed-point wave functions:

$$
\left(d \nu_{4}\right)\left(g_{0}, g_{1}, g_{2}, g_{3}, g_{4}, g_{5}\right) \equiv \frac{\nu_{4}\left(g_{1}, g_{2}, g_{3}, g_{4}, g_{5}\right) \nu_{4}\left(g_{0}, g_{1}, g_{3}, g_{4}, g_{5}\right) \nu_{4}\left(g_{0}, g_{1}, g_{2}, g_{3}, g_{5}\right)}{\nu_{4}\left(g_{0}, g_{2}, g_{3}, g_{4}, g_{5}\right) \nu_{4}\left(g_{0}, g_{1}, g_{2}, g_{4}, g_{5}\right) \nu_{4}\left(g_{0}, g_{1}, g_{3}, g_{2}, g_{4}\right)}=1
$$

Similar to the $1 \mathrm{D}$ and $2 \mathrm{D}$ cases, we can use SLU to redefine the basis state $\left|\left\{g_{l}\right\}\right\rangle$ as

$$
\left|\left\{g_{l}\right\}\right\rangle^{\prime}=U_{\mu_{3}}\left|\left\{g_{l}\right\}\right\rangle=\prod_{\langle i j k l\rangle} \mu_{3}\left(g_{i}, g_{j}, g_{k}, g_{l}\right)^{s_{\langle i j k l\rangle}}\left|\left\{g_{l}\right\}\right\rangle,
$$

where $s_{\langle i j k l\rangle}= \pm 1$ denotes the orientation of the tetrahedron. One finds that the phase factor in Eq. (48) becomes

$$
\nu_{4}^{\prime}\left(g_{0}, g_{1}, g_{2}, g_{3}, g_{4}\right)=\nu_{4}\left(g_{0}, g_{1}, g_{2}, g_{3}, g_{4}\right) \frac{\mu_{3}\left(g_{1}, g_{2}, g_{3}, g_{4}\right) \mu_{3}\left(g_{0}, g_{1}, g_{3}, g_{4}\right) \mu_{3}\left(g_{0}, g_{1}, g_{2}, g_{3}\right)}{\mu_{3}\left(g_{0}, g_{2}, g_{3}, g_{4}\right) \mu_{3}\left(g_{0}, g_{1}, g_{2}, g_{4}\right)}
$$

So the elements $\nu_{4}$ in the same group cohomology class in $H^{4}\left[G, U(1)_{T}\right]$ correspond to the same 3D BSPT phase.

We can also use the above moves to construct a 3D commuting-projector parent Hamiltonian. Each term of the Hamiltonian is a sequence of 3D moves that changes the label of a vertex from $g_{*}$ to $g_{*}^{\prime}$. All the terms commute with each other, because the wave function is at the fixed point and $\nu_{4}$ satisfy the 4-cocycle condition Eq. (53).

Finally, we notice that for antiunitary symmetry, e.g., time-reversal symmetry, the above construction and classification scheme is not complete. It has been pointed out [8] that the decoration of the $E_{8}$ state on the $G$-symmetry domain walls gives rise to new BSPT states beyond group cohomology classification. Apparently, the data $H^{1}\left(G, \mathbb{Z}_{T}\right)$ classify such a decorating pattern and the corresponding additional BSPT states. Since $H^{1}\left(G, \mathbb{Z}_{T}\right)$ is trivial for all unitary symmetry groups $G$ and $H^{1}\left(\mathbb{Z}_{2}^{T}, \mathbb{Z}_{T}\right)=\mathbb{Z}_{2}$ for the (antiunitary) time-reversal symmetry, we understand why the beyond group cohomology BSPT phases arise only for antiunitary symmetry. Thus, we conclude that the two cohomology groups of the symmetry group $G, H^{1}\left(G, \mathbb{Z}_{T}\right)$ and $H^{4}\left[G, U(1)_{T}\right]$, give rise to a complete classification of BSPT phases in $3 \mathrm{D}$.

\section{FSLU TRANSFORMATION AND FSPT PHASES}

\section{A. Fermionic symmetric local unitary transformations}

In Ref. [72], it is shown that fermionic local unitary (FLU) transformations can be used to define and classify intrinsic topological phases for interacting fermion systems. The Fock space structure and fermion parity conservation symmetry of fermion systems can be naturally encoded into FLU transformations. It is well known that the finite-time FLU evolution is closely related to fermionic quantum circuits with finite depth, which is defined through piecewise FLU operators. A piecewise FLU operator has the form $U_{p w l}=\prod_{i} e^{-i H_{f}(i)} \equiv \prod_{i} U(\boldsymbol{i})$, where $H_{f}(\boldsymbol{i})$ is a fermionic Hermitian operator and $U(\boldsymbol{i})$ is the corresponding fermionic unitary operator defined in Fock space that preserves fermion parity (e.g., contains an even number of fermion creation and annihilation operators) and acts on a region labeled by $i$. Note that regions labeled by different $i$ 's are not overlapping. We further require that the size of each region is less than some finite number $l$. The unitary operator $U_{p w l}$ defined in this way is called a piecewise fermionic local unitary operator with range $l$. A fermion quantum circuit with depth $M$ is given by the product of $M$ piecewise fermionic local unitary operators: $U_{\text {circ }}^{M}=U_{p w l}^{(1)} U_{p w l}^{(2)} \ldots U_{p w l}^{(M)}$. It is believed that any FLU evolution can be simulated with a constant-depth fermionic quantum circuit and vice versa. Therefore, the equivalence relation between gapped states in interacting fermion systems can be rewritten in terms of constant-depth fermionic quantum circuits:

$$
|\psi(1)\rangle \sim|\psi(0)\rangle \quad \text { iff }|\psi(1)\rangle=U_{\text {circ }}^{M}|\psi(0)\rangle .
$$

Thus, we can use the term FLU transformation to refer to both FLU evolution and constant-depth fermionic quantum circuit. From the definition of the FSPT state, it is easy to see that (in the absence of global symmetry) 


$$
\left.|\mathrm{FSPT}\rangle=U_{\text {circ }}^{M} \mid \text { trivial }\right\rangle .
$$

Namely, an FSPT state can be connected to a trivial state (e.g., a product state) vial FLU transformation (in the absence of global symmetry). Similar to the BSPT case, Eq. (55) implies that the support space of any FSPT in region $A$ must be one dimensional. This constraint is simply because a trivial state (e.g., a product state) has a onedimensional support space, and any FSPT state becomes a product state via a proper local basis change (induced by a FLU transformation).

In the presence of global symmetry, we can further introduce the notion of invertible FSLU transformations to define and classify FSPT phases in interacting fermion systems. By FSLU transformation, we mean that the corresponding piecewise FLU operator is invariant under total symmetry group $G_{f}$.

\section{B. Layers of degrees of freedom}

There are at most four layers of degrees of freedom in total in our fixed-point wave function of the FSPT state (up to four spacetime dimensions). The bosonic states are always at the vertices. And the fermionic degrees of freedom (complex fermions, Majorana fermions, and 2D $p+i p$ chiral superconductors) are decorated on the intersecting submanifold of the bosonic state. In summary, the degrees of freedom of our FSPT states are [73]

(i) $\left|G_{b}\right|$ level bosonic (spin) state $\left|g_{i}\right\rangle\left(g_{i} \in G_{b}\right)$ on each vertex $i$

(ii) $\left|G_{b}\right|$ species of complex fermions $c_{i j \ldots k}^{\sigma}\left(\sigma \in G_{b}\right)$ on each codimension-0 simplex $\langle i j \ldots k\rangle$;

(iii) $\left|G_{b}\right|$ species of Majorana fermions $\gamma_{i j \ldots k, A}^{\sigma}$ and $\gamma_{i j \ldots k, B}^{\sigma}\left(\sigma \in G_{b}\right)$, which come from complex fermions $a_{i j \ldots k}^{\sigma}=\left(\gamma_{i j \ldots k, A}^{\sigma}+i \gamma_{i j \ldots k, B}^{\sigma}\right) / 2$, on the two sides of each codimension-1 simplex $\langle i j \ldots k\rangle$;

(iv) $\left|G_{b}\right|$ species of $2 \mathrm{D} p+i p$ chiral superconductors (may have several copies) on the dual surface of each codimension-2 simplex. The chiral Majorana modes along the edge of the dual surface are labeled by $\psi_{i j \ldots k, L}^{\sigma}$ or $\psi_{i j \ldots k, R}^{\sigma}$ depending on the chirality.

The above degrees of freedom have different symmetry transformation rules. The symmetry transformation of $G_{b}$ on the bosonic state is the same as that in the BSPT states $\left(g \in G_{b}\right)$ :

$$
U(g)\left|g_{i}\right\rangle=\left|g g_{i}\right\rangle
$$

For complex fermions, we choose the symmetry transformations under $G_{b}$ to be

$$
U(g) c_{i j \ldots k}^{\sigma} U(g)^{\dagger}=(-1)^{\omega_{2}(g, \sigma)} c_{i j \ldots k}^{g \sigma} .
$$

The symmetry transformation rules of Majorana fermions $\gamma_{i j \ldots k, A / B}^{\sigma}$ are induced by the transformation of complex fermion $a_{i j \ldots k}^{\sigma}$ :

$$
\begin{gathered}
U(g) a_{i j \ldots k}^{\sigma} U(g)^{\dagger}=(-1)^{\omega_{2}(g, \sigma)} a_{i j \ldots k}^{g \sigma}, \\
U(g) \gamma_{i j \ldots k, A}^{\sigma} U(g)^{\dagger}=(-1)^{\omega_{2}(g, \sigma)} \gamma_{i j \ldots k, A}^{g \sigma}, \\
U(g) \gamma_{i j \ldots k, B}^{\sigma} U(g)^{\dagger}=(-1)^{\omega_{2}(g, \sigma)+s_{1}(g)} \gamma_{i j \ldots k, B}^{g \sigma} .
\end{gathered}
$$

And the symmetry transformations of chiral Majorana modes on the boundary of decorated $p+i p$ superconductors are chosen to be

$$
\begin{gathered}
U(g) \psi_{i j \ldots k, R}^{\sigma} U(g)^{\dagger}=(-1)^{\omega_{2}(g, \sigma)} \psi_{i j \ldots k, g(R)}^{g \sigma}, \\
U(g) \psi_{i j \ldots k, L}^{\sigma} U(g)^{\dagger}=(-1)^{\omega_{2}(g, \sigma)+s_{1}(g)} \psi_{i j \ldots k, g(L)}^{g \sigma} .
\end{gathered}
$$

We discuss more about why we choose the transformation rules for Majorana modes in Sec. VIF 2.

In this way, the $G_{b}$ species of fermions span a space that supports a projective representation of $G_{b}$ with coefficient $P_{f}^{\omega_{2}}$ :

$$
U(g) U(h)=P_{f}^{\omega_{2}(g, h)} U(g h),
$$

with $P_{f}=-1$ when acting on fermion parity odd states. We note that the projective representation $U$ of $G_{b}$ is equivalent to a linear representation $\tilde{U}$ of $G_{f}$ by

$$
\tilde{U}\left(P_{f}^{n} g\right):=P_{f}^{n} U(g)
$$

One can check directly that $\tilde{U}$ is indeed a genuine linear representation of $G_{f}$ :

$$
\tilde{U}\left(P_{f}^{m} g\right) \tilde{U}\left(P_{f}^{n} h\right)=\tilde{U}\left(P_{f}^{m} g \cdot P_{f}^{n} h\right),
$$

where the dot product in $G_{f}$ is defined in Eq. (2).

In the previous constructions of the FSPT state for $G_{f}=\mathbb{Z}_{2}^{f} \times G_{b}$, we put only one species of fermions on each simplex $[52,53]$. They transform trivially under the action of $G_{b}$ (and $G_{f}$ ). To construct a FSPT state for $G_{f}=\mathbb{Z}_{2}^{f} \times_{\omega_{2}} G_{b}$, we need a projective representation of $G_{b}$ with coefficient $\omega_{2}$ to make it a linear representation of $G_{f}$. We choose the canonical $\left|G_{b}\right|$-dimensional projective representation Eq. (57), which can be constructed for arbitrary finite symmetry group $G_{b}$. Although there are $\left|G_{b}\right|$ species fermions $c_{i j \ldots k}^{\sigma}\left(\sigma \in G_{b}\right)$ on each simplex $\langle i j \ldots k\rangle$, (at most) only one of them $c_{i j \ldots k}^{g_{i}}$ is decorated or in 
the occupied state, and all other fermion species $c_{i j \ldots k}^{\sigma}$ $\left(\sigma \neq g_{i}\right)$ are in the vacuum states. In such a way, the FSPT constructions for symmetry group $G_{f}=\mathbb{Z}_{2}^{f} \times G_{b}$ can be generalized to the case of $G_{f}=\mathbb{Z}_{2}^{f} \times_{\omega_{2}} G_{b}$.

\section{Symmetry conditions and consistency equations}

Since we are constructing FSPT states, the $F$ moves should be compatible with the symmetry action $U(g)$ defined in Sec. III B. To be more precise, let us consider the following two-dimensional commuting diagram:

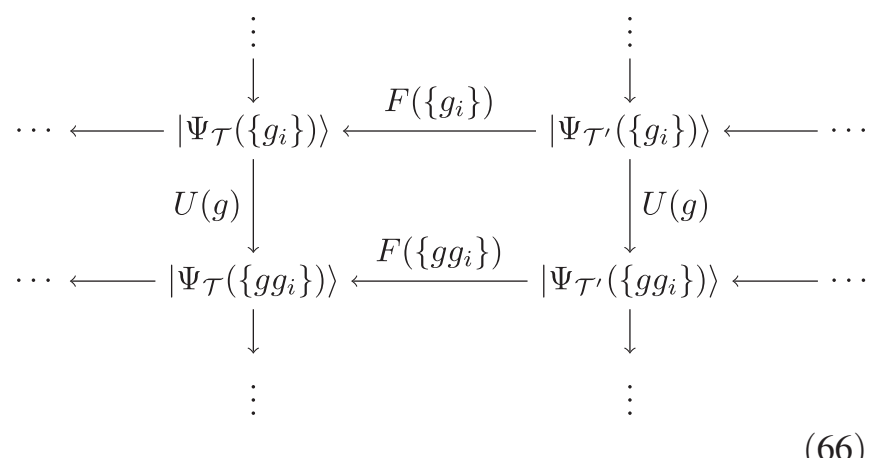

where the horizontal $F$ move changes the triangulations of the spatial manifold and the vertical symmetry action $U(g)$ changes the bosonic degrees of freedom from $\left\{g_{i}\right\}$ to $\left\{g g_{i}\right\}$ (similar for the fermionic ones). The outside parts "..." correspond to other triangulations of the spatial manifold and other symmetry actions on the states. This two-dimensional diagram should commute for an arbitrary horizontal Pachner move of arbitrary triangulations and vertical symmetry action with arbitrary $g \in G_{b}$. The requirement of symmetric fixed-point wave function implies the following conditions.

(1) The diagram Eq. (66) commutes, i.e.,

$$
F\left(\left\{g g_{i}\right\}\right)=U(g) F\left(\left\{g_{i}\right\}\right) U(g)^{\dagger}, \quad \forall g \in G_{b} .
$$

(2) The vertical direction of diagram Eq. (66) should form a projective representation of $G_{b}$ with coefficient $\omega_{2}$, i.e., $U(g) U(h)=P_{f}^{\omega_{2}(g, h)} U(g h)$ when acting on these states. This projective representation of $G_{b}$ induces a linear representation of $G_{f}$ by Eq. (64).

(3) The horizontal direction of diagram Eq. (66) should satisfy some coherence equation, which is known as a super (fermionic) pentagon equation in 2D. In the FSPT setting, it is a twisted cocycle equation.

For the triangulations of $d$-dimensional space manifold, the Pachner move involves $d+2$ vertices. So the basic $F$ move can be denoted by $F\left(g_{0}, g_{1}, \ldots, g_{d+1}\right)$. With the help of Eq. (67), we can obtain the generic $F$ move

$$
\begin{aligned}
F\left(g_{0}, g_{1}, \ldots, g_{d+1}\right) & ={ }^{g_{0}} F\left(e, g_{0}^{-1} g_{1}, \ldots, g_{0}^{-1} g_{d+1}\right) \\
& =U\left(g_{0}\right) F\left(e, g_{0}^{-1} g_{1}, \ldots, g_{0}^{-1} g_{d+1}\right) U\left(g_{0}\right)^{\dagger},
\end{aligned}
$$

provided that we define the standard $F$ symbol with the first argument being the identity element $e \in G_{b}$. Using this definition of the $F$ move, Eq. (67) is automatically satisfied. This result is because of the following commuting diagram:

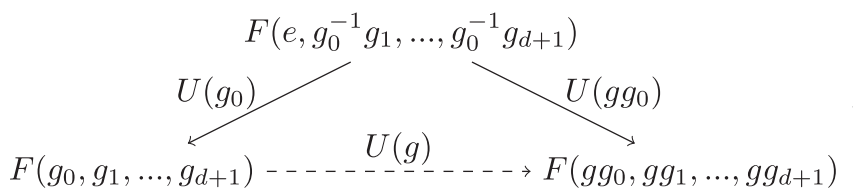

We can deduce the dashed arrow $U(g)$ from solid arrows $U\left(g_{0}\right)$ and $U\left(g g_{0}\right)$, due to $U(g) U(h)=P_{f}^{\omega_{2}(g, h)} U(g h)$ and the fermion parity even property of the $F$ operators.

\section{FIXED-POINT WAVE FUNCTION AND CLASSIFICATION OF FSPT STATES IN 1D}

In this section, we give the explicit constructions and classifications of 1D FSPT states. The fixed-point wave functions are obtained by decorating complex fermions to BSPT states consistently. Formally, the wave function is a superposition of all basis states $\left|\left\{g_{i}\right\}\right\rangle$ with legitimate decorations:

$|\Psi\rangle=\sum_{\text {all conf. }} \Psi(\curvearrowleft \circ \cdots \cdots)|\curvearrowleft \ldots \cdots \cdots\rangle$

The basis state $\left|\left\{g_{i}\right\}\right\rangle$ is a state (with vertex $i$ labeled by $g_{i} \in G_{b}$ ) decorated by complex fermions $c_{i j}^{\sigma}$ at link $\langle i j\rangle$. The constructed fixed-point wave function $|\Psi\rangle$ should be both symmetric and topological (invariant under retriangulations of the lattice). As shown below, these constraints would give us the consistency conditions for the 1D FSPT classifications summarized in Eqs. (8)-(11).

We note that the 1D Kitaev chain is a fermionic invertible topological order. Since it does not need any bosonic symmetry protection ( $\mathbb{Z}_{2}^{f}$ cannot be broken), we do not consider it as a FSPT state. The Kitaev chain layer is useful when considering it as the ASPT on the boundary of a 2D FSPT state. The 2D classification data $n_{2}$ is trivialized. 
This section is organized as follows. The two layers of degrees of freedom (bosonic states and complex fermions) are introduced in Sec. IV A. In Sec. IV B, we propose the procedures of symmetrically decorating complex and Majorana fermions to BSPT states. Then, the construction and the consistency equations of the FSLU transformations are discussed in Secs. IV C and IV D, respectively.

\section{A. Two layers of degrees of freedom}

The basic idea to construct FSPT states is to decorate complex fermions to the BSPT states. Therefore, there are two layers of degrees of freedom, including the bosonic ones, in the 1D lattice model:

(i) $\left|G_{b}\right|$ level bosonic (spin) state $\left|g_{i}\right\rangle\left(g_{i} \in G_{b}\right)$ on each vertex $i$ and

(ii) $\left|G_{b}\right|$ species of complex fermions $c_{i j}^{\sigma}\left(\sigma \in G_{b}\right)$ at the center of each link $\langle i j\rangle$.

These degrees of freedom are summarized in one unit cell in the following:

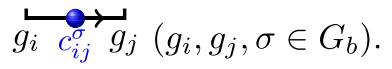

Here, we choose the link direction from vertex $i$ to vertex $j$ (from left to right). The vertices are labeled by $g_{i}$ and $g_{j}$, which are elements of $G_{b}$. The blue ball is the decorated complex fermion $c_{i j}^{\sigma}\left(\sigma \in G_{b}\right)$ at the center of link $\langle i j\rangle$.

The symmetry transformations of these degrees of freedom under $G_{b}$ are the same as the discussions in Sec. III B. To be more specific to the 1D case, we summarize them as $\left(g, g_{i}, \sigma \in G_{b}\right)$

$$
\begin{gathered}
U(g)\left|g_{i}\right\rangle=\left|g g_{i}\right\rangle, \\
U(g) c_{i j}^{\sigma} U(g)^{\dagger}=(-1)^{\omega_{2}(g, \sigma)} c_{i j}^{g \sigma} .
\end{gathered}
$$

While the bosonic degrees of freedom on each vertex form a linear representation of $G_{b}$, the complex fermions form a projective representation of $G_{b}$ with coefficient $(-1)^{\omega_{2}}$. In this way, they all transform linearly under the action of $G_{f}$ defined by Eq. (64).

Although there are $\left|G_{b}\right|$ species of complex fermions in the Hilbert space of the system, we see later that (at most) only one of them is decorated nontrivially in the fixed-point wave function. If we consider the case of $\omega_{2}=0$ (i.e., $G_{f}=\mathbb{Z}_{2}^{f} \times G_{b}$ ), the symmetry transformation rules are independent of group element label $\sigma$ of the fermions [see Eq. (73)]. Therefore, we can reduce these $\left|G_{b}\right|$ species of fermions to only one species without a group element label. The resulting states are exactly the ones studied in Refs. [52,53].

\section{B. Decoration of complex fermions}

In the group cohomology theory of BSPT phases [4], the fixed-point wave functions are constructed as superpositions of all basis state $\left|\left\{g_{i}\right\}\right\rangle$. The coefficients in front of these basis states are $U(1)$-valued cocycles. To construct FSPT states, we introduce the fermionic degrees of freedom associated to the basis states in the previous section. In the following, we discuss the detailed procedures of systematically decorating complex fermions. These decorations should be designed to be symmetric under symmetry actions.

The complex fermion decoration is specified by a $\mathbb{Z}_{2}$-valued 1-cochain $n_{1} \in C^{1}\left(G_{b}, \mathbb{Z}_{2}\right)$, which is the first classification data for 1D FSPT phases. If $n_{1}\left(g_{i}, g_{j}\right)=0$, all the modes of complex fermions $c_{i j}^{\sigma}\left(\sigma \in G_{b}\right)$ at link $\langle i j\rangle$ are unoccupied (shown by blue circles in figures). On the other hand, if $n_{1}\left(g_{i}, g_{j}\right)=1$, exactly one complex fermion $c_{i j}^{g_{i}}$ is decorated at the center of the oriented link $\langle i j\rangle$ (shown by filled blue balls in figures), and all other complex fermions $c_{i j}^{\sigma}\left(\sigma \neq g_{i}\right)$ are still in vacuum states.

The above complex fermion decoration rule is $G_{b}$ symmetric. Under a $U(g)$ action, the vertex labels of link $\langle i j\rangle$ become $g g_{i}$ and $g g_{j}$. According to the decoration rule, the decorated complex fermion [if $n_{1}\left(g g_{i}, g g_{j}\right)=$ $\left.n_{1}\left(g_{i}, g_{j}\right)=1\right]$ should be $c_{i j}^{g g_{i}}$, which is exactly the complex fermion $c_{i j}^{g_{i}}$ by a $U(g)$ action.

\section{C. $\boldsymbol{F}$ moves}

For a fixed triangulation of spatial manifold, we can decorate complex fermions symmetrically as discussed above. However, we want to construct fixed-point wave functions that are invariant under retriangulation of the space. To connect different triangulations, there are FSLU transformations for each Pachner move. For the 1D lattice, there is essentially only one Pachner move given by

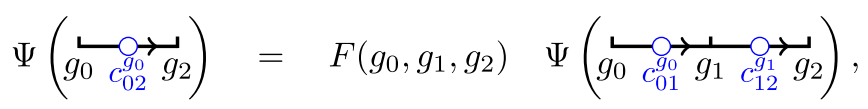

where the FSLU $F$ operator is defined as

$$
\begin{aligned}
F\left(g_{0}, g_{1}, g_{2}\right)= & \left|G_{b}\right|^{1 / 2} \nu_{2}\left(g_{0}, g_{1}, g_{2}\right)\left(c_{02}^{g_{0}}\right)^{\dagger n_{1}\left(g_{0}, g_{2}\right)} \\
& \times\left(c_{01}^{g_{0}}\right)^{n_{1}\left(g_{0}, g_{1}\right)}\left(c_{12}^{g_{1}}\right)^{n_{1}\left(g_{1}, g_{2}\right)} .
\end{aligned}
$$

In the above expression of the $F$ symbol, $\left|G_{b}\right|^{1 / 2}$ is the normalization factor, because the number of lattice sites is reduced by one. $\nu_{2}\left(g_{0}, g_{1}, g_{2}\right)$ is a $U(1)$ phase factor depending on three group elements of $G_{b}$. For BSPT states, the $F$ operator has only these two bosonic factors. 
For FSPT states, however, there are complex fermion terms of the form $c^{\dagger} c c$. The complex fermion term annihilates the possibly decorated (depending on $n_{1}$ ) complex fermions $c_{12}^{g_{1}}$ and $c_{01}^{g_{0}}$ on the two links in the right-hand-side state and create a new complex fermion $c_{02}^{g_{0}}$ at the center of link $\langle 02\rangle$ in the left-hand-side state.

As discussed above, the $F$ move should be a FSLU operator. Therefore, it should be both fermion parity even and symmetric under $G_{b}$ action. These two conditions give us several consistency equations for the classification data $n_{1}$ and $\nu_{2}$.

\section{Fermion parity conservation}

Since the complex fermions are decorated according to $n_{1}\left(g_{i}, g_{j}\right)$, the complex fermion parity change of the $F$ move Eq. (74) is

$$
\begin{aligned}
\Delta P_{f}^{c}(012) & =(-1)^{n_{1}\left(g_{0}, g_{2}\right)+n_{1}\left(g_{0}, g_{1}\right)+n_{1}\left(g_{1}, g_{2}\right)} \\
& =(-1)^{d n_{1}\left(g_{0}, g_{1}, g_{2}\right)} .
\end{aligned}
$$

As a result, the conservation of fermion parity under the $F$ move enforces the condition

$$
d n_{1}=0 \quad(\bmod 2)
$$

which is the cocycle equation for the decoration data $n_{1}$.

\section{Symmetry condition}

The $F$ move should also be consistent with the symmetry actions [see Eq. (66)]. In 1D, we have the following commuting diagram:

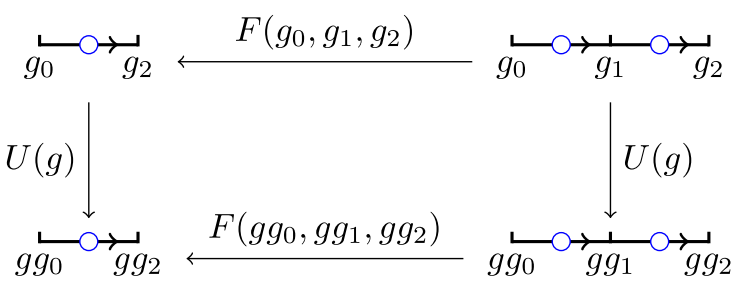

or the symmetry condition for $F$ operators:

$$
F\left(g g_{0}, g g_{1}, g g_{2}\right)=U(g) F\left(g_{0}, g_{1}, g_{2}\right) U(g)^{\dagger} .
$$

As discussed in Sec. IIIC, the above equation can be viewed as a definition of the generic $F\left(g_{0}, g_{1}, g_{2}\right)$ in terms of the standard $F$ move $F\left(e, g_{0}^{-1} g_{1}, g_{0}^{-1} g_{2}\right)$ :

$$
\begin{aligned}
F\left(g_{0}, g_{1}, g_{2}\right) & ={ }_{0} F\left(e, g_{0}^{-1} g_{1}, g_{0}^{-1} g_{2}\right) \\
& :=U\left(g_{0}\right) F\left(e, g_{0}^{-1} g_{1}, g_{0}^{-1} g_{2}\right) U\left(g_{0}\right)^{\dagger} .
\end{aligned}
$$

Therefore, we need only to fix the expression of the standard $F$ move $F\left(e, g_{0}^{-1} g_{1}, g_{0}^{-1} g_{2}\right)$, and all other nonstandard $F$ moves are obtained by a symmetry action on the standard one. The explicit expression of the standard $F$ move $F\left(e, g_{0}^{-1} g_{1}, g_{0}^{-1} g_{2}\right)$ is given by

$$
F\left(e, g_{0}^{-1} g_{1}, g_{0}^{-1} g_{2}\right)=\left|G_{b}\right|^{1 / 2} \nu_{2}\left(g_{0}^{-1} g_{1}, g_{1}^{-1} g_{2}\right)\left(c_{02}^{e}\right)^{\dagger n_{1}\left(e, g_{0}^{-1} g_{2}\right)}\left(c_{01}^{e}\right)^{n_{1}\left(e, g_{0}^{-1} g_{1}\right)}\left(c_{12}^{g_{0}^{-1} g_{1}}\right)^{n_{1}\left(g_{0}^{-1} g_{1}, g_{0}^{-1} g_{2}\right)} \text {. }
$$

Note that the $U(1)$ coefficient in the standard $F$ move is chosen to be the inhomogeneous cochain $\nu_{2}\left(g_{0}^{-1} g_{1}, g_{1}^{-1} g_{2}\right):=\nu_{2}\left(e, g_{0}^{-1} g_{1}, g_{0}^{-1} g_{2}\right)$. And we do not impose the condition " $\nu_{2}\left(g g_{0}, g g_{1}, g g_{2}\right)=\nu_{2}\left(g_{0}, g_{1}, g_{2}\right)$ " a priori. In fact, as shown below, this condition does not hold, in general.

We can apply a $U\left(g_{0}\right)$ action on the standard $F$ move Eq. (81) and compare it with the generic expression Eq. (75). The symmetry conditions for $n_{1}$ and $\nu_{2}$ are

$$
\begin{gathered}
n_{1}\left(g_{0}, g_{1}\right)=n_{1}\left(e, g_{0}^{-1} g_{1}\right)=n_{1}\left(g_{0}^{-1} g_{1}\right), \\
\nu_{2}\left(g_{0}, g_{1}, g_{2}\right)={ }^{g_{0}} \nu_{2}\left(g_{0}^{-1} g_{1}, g_{1}^{-1} g_{2}\right)=\nu_{2}\left(g_{0}^{-1} g_{1}, g_{1}^{-1} g_{2}\right)^{1-2 s_{1}\left(g_{0}\right)} \cdot(-1)^{\left(\omega_{2} \smile n_{1}\right)\left(g_{0}, g_{0}^{-1} g_{1}, g_{1}^{-1} g_{2}\right)},
\end{gathered}
$$

respectively, where the term $(-1)^{\omega_{2} \smile n_{1}}$ comes from the symmetry transformations of $c_{12}^{g_{0}^{-1} g_{1}}$. We also introduce new notations to relate the homogeneous cochain $\nu_{2}\left(g_{0}, g_{1}, g_{2}\right)={ }^{g_{0}} \nu_{2}\left(g_{0}^{-1} g_{1}, g_{1}^{-1} g_{2}\right)$ and the inhomogeneous cochain $\nu_{2}\left(g_{0}^{-1} g_{1}, g_{1}^{-1} g_{2}\right)={ }^{e} \nu_{2}\left(g_{0}^{-1} g_{1}, g_{1}^{-1} g_{2}\right)$. In the following, when we write the cochain $\nu_{d}$ without arguments, we always mean the inhomogeneous one, i.e., $\nu_{d}={ }^{e} \nu_{d}$.

\section{Associativity and twisted cocycle equations}

The $F$ move reduces three vertices on the lattice to two vertices. If one considers reducing four vertices to two vertices, there are two inequivalent ways to do that. The final results should be independent of the two ways. This constraint gives us the consistency equation for Pachner moves: 


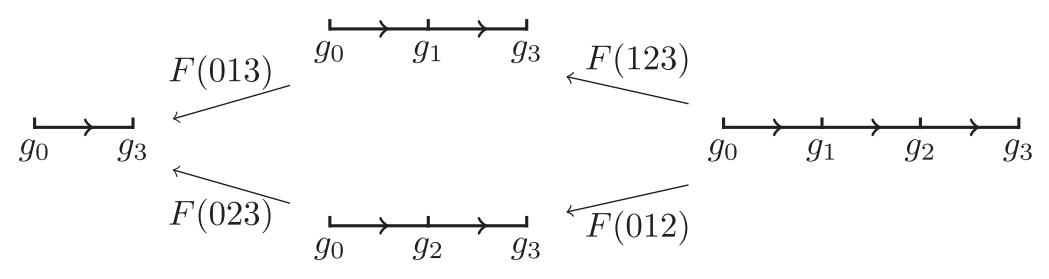

In terms of $F$ operators, the above commuting diagram means

$$
F\left(g_{0}, g_{2}, g_{3}\right) \cdot F\left(g_{0}, g_{1}, g_{2}\right)=F\left(g_{0}, g_{1}, g_{3}\right) \cdot F\left(g_{1}, g_{2}, g_{3}\right) \text {. }
$$

Similar to the standard $F$ symbol, which can be used to derive all other nonstandard ones by a symmetry action, we can also assume $g_{0}=e$ in the above equation. All other consistency equations with generic $g_{0}$ can be deduced from this standard equation by a $U\left(g_{0}\right)$ action. Therefore, we need only to consider the consistency equation

$$
\begin{aligned}
F\left(e, g_{0}^{-1} g_{2}, g_{0}^{-1} g_{3}\right) \cdot F\left(e, g_{0}^{-1} g_{1}, g_{0}^{-1} g_{2}\right) & =F\left(e, g_{0}^{-1} g_{1}, g_{0}^{-1} g_{3}\right) \cdot F\left(g_{0}^{-1} g_{1}, g_{0}^{-1} g_{2}, g_{0}^{-1} g_{3}\right) \\
& =F\left(e, g_{0}^{-1} g_{1}, g_{0}^{-1} g_{3}\right) \cdot\left(g_{0}^{-1} g_{1}\right) F\left(e, g_{1}^{-1} g_{2}, g_{1}^{-1} g_{3}\right)
\end{aligned}
$$

The above equation is simpler than the generic one [Eq. (85)], since only the last $F$ symbol is nonstandard.

Substituting the standard $F$ move [Eq. (81)], the consistency equation [Eq. (86)] becomes

$$
\nu_{2}\left(g_{0}^{-1} g_{2}, g_{2}^{-1} g_{3}\right) \nu_{2}\left(g_{0}^{-1} g_{1}, g_{1}^{-1} g_{2}\right)=\nu_{2}\left(g_{0}^{-1} g_{1}, g_{1}^{-1} g_{3}\right)\left[\nu_{2}\left(g_{1}^{-1} g_{2}, g_{2}^{-1} g_{3}\right)\right]^{1-2 s_{1}\left(g_{0}^{-1} g_{1}\right)}(-1)^{\left(\omega_{2} \smile n_{1}\right)\left(g_{0}^{-1} g_{1}, g_{1}^{-1} g_{2}, g_{2}^{-1} g_{3}\right)},
$$

where the last term $(-1)^{\omega_{2} \sim n_{1}}$ comes from the $U\left(g_{0}^{-1} g_{1}\right)$ action on $F\left(e, g_{1}^{-1} g_{2}, g_{1}^{-1} g_{3}\right)$ [see Eq. (82)]. Note that the complex fermions do not contribute any fermion signs. So we have the twisted cocycle equation for inhomogeneous $\nu_{2}$ :

$$
d \nu_{2}=\mathcal{O}_{3}\left[n_{1}\right]
$$

with obstruction function

$$
\mathcal{O}_{3}\left[n_{1}\right]=(-1)^{\omega_{2} \smile n_{1}} .
$$

In summary, the associativity condition for the $F$ moves in $1 \mathrm{D}$ gives us the twisted cocycle equation [Eqs. (88) and (89)] for inhomogeneous cochain $\nu_{2}$.

\section{E. Classification of 1D FSPT phases}

The general classification of 1D FSPT phases is as follows. We first calculate the cohomology groups $H^{1}\left(G_{b}, \mathbb{Z}_{2}\right)$ and $H^{2}\left[G_{b}, U(1)_{T}\right]$. For each $n_{1} \in H^{1}\left(G_{b}, \mathbb{Z}_{2}\right)$, we solve the twisted cocycle equation [Eq. (10)] for $\nu_{2}$. If $\nu_{2}$ is in the trivialization subgroup $\Gamma^{2}$ in Eq. (11), it is known to be trivialized by complex fermion decoration [52]; see Appendix A for more details. So the obstruction-free $n_{1}$ and trivialization-free $\nu_{2}$ fully classify the 1D FSPT phases.

We note that we can use the FSLU transformations to construct the commuting-projector parent Hamiltonians.
Each term of the Hamiltonian is a sequence of fermionic $F$ moves that changes the label of a vertex from $g_{*}$ to $g_{*}^{\prime}$. All the terms commute with each other, for our FSPT wave function is at the fixed point.

\section{FIXED-POINT WAVE FUNCTION AND CLASSIFICATION OF FSPT STATES IN 2D}

In this section, we construct and classify FSPT states in two spatial dimensions. The fixed-point wave function is again a superposition of all basis states $\left|\left\{g_{i}\right\}\right\rangle$ with fermion decorations. The basis state $\left|\left\{g_{i}\right\}\right\rangle$ is a state (with vertex $i$ labeled by $g_{i} \in G_{b}$ ) decorated by complex fermions $c_{i j}^{\sigma}$ at link $\langle i j\rangle$ and Majorana fermions $\gamma_{i, A}^{\sigma}$ and $\gamma_{i, B}^{\sigma}$ near vertex $i$ according to several designed rules. So the fixed-point wave function looks like

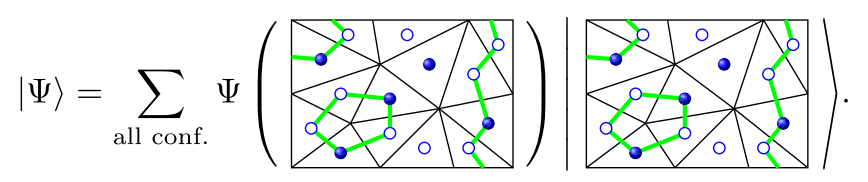

\section{A. Three layers of degrees of freedom}

In 2D, we decorate two layers of fermionic degrees of freedom to the BSPT states. Therefore, there are three 
layers of degrees of freedom, including the bosonic ones, in our 2D triangulation lattice model:

(i) $\left|G_{b}\right|$ level bosonic (spin) state $\left|g_{i}\right\rangle\left(g_{i} \in G_{b}\right)$ on each vertex $i$

(ii) $\left|G_{b}\right|$ species of complex fermions $c_{i j k}^{\sigma}\left(\sigma \in G_{b}\right)$ at the center of each triangle $\langle i j k\rangle$, and

(iii) $\left|G_{b}\right|$ species of complex fermions (split to Majorana fermions) $a_{i j}^{\sigma}=\left(\gamma_{i j, A}^{\sigma}+i \gamma_{i j, B}^{\sigma}\right) / 2\left(\sigma \in G_{b}\right)$ on the two sides of each link $\langle i j\rangle$.

These three layers of degrees of freedom are summarized in one triangle in the following figure:

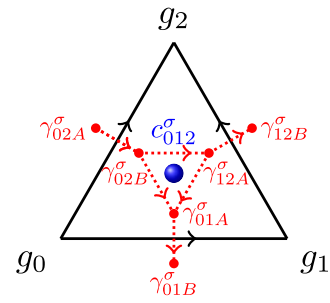

Here, the three vertices of the triangle are labeled by $g_{0}, g_{1}, g_{2} \in G_{b}$. The blue ball is the complex fermion $c_{012}^{\sigma}$ $\left(\sigma \in G_{b}\right)$ at the center of triangle $\langle 012\rangle$. Red dots represent Majorana fermions $\gamma_{i j, A}^{\sigma}$ and $\gamma_{i j, B}^{\sigma}\left(\sigma \in G_{b}\right)$ on the two sides of link $\langle i j\rangle$.

The symmetry transformations of these degrees of freedom under $G_{b}$ are summarized then as follows $\left(g, g_{i}, \sigma \in G_{b}\right)$ :

$$
\begin{gathered}
U(g)\left|g_{i}\right\rangle=\left|g g_{i}\right\rangle, \\
U(g) c_{i j k}^{\sigma} U(g)^{\dagger}=(-1)^{\omega_{2}(g, \sigma)} c_{i j k}^{g \sigma}, \\
U(g) \gamma_{i j, A}^{\sigma} U(g)^{\dagger}=(-1)^{\omega_{2}(g, \sigma)} \gamma_{i j, A}^{g \sigma}, \\
U(g) \gamma_{i j, B}^{\sigma} U(g)^{\dagger}=(-1)^{\omega_{2}(g, \sigma)+s_{1}(g)} \gamma_{i j, B}^{g \sigma} .
\end{gathered}
$$

As in the 1D case, the bosonic degrees of freedom form a linear representation of $G_{b}$ (and $G_{f}$ ). On the other hand, the fermion modes support projective representations of $G_{b}$ with coefficient $(-1)^{\omega_{2}}$ and linear representations of $G_{f}$ defined by Eq. (64).

In the simpler case of $G_{f}=\mathbb{Z}_{2}^{f} \times G_{b}$, all the flavors of fermions have the same transformation rule for different group element label $\sigma$. So we can suppress the species labels. This result is again the previous group supercohomology models [52,53].

\section{B. Decorations of fermion layers}

In this section, we give a systematic procedure to decorate Kitaev chains and complex fermions to the basis state $\left|\left\{g_{i}\right\}\right\rangle$ labeled by $g_{i} \in G_{b}$ for each vertex $i$. Similar to the 1D case, we decorate (at most) only one species of fermions to the state, although the Hilbert space is spanned by $\left|G_{b}\right|$ copies of fermions. Again, the decorations should be designed to respect the symmetry.

\section{Kitaev chain decoration}

The Kitaev chain decoration in 2D is similar to the constructions in the pioneering works Refs. [74,75]. However, we adopt the more general procedures in Ref. [53], which can deal with arbitrary triangulations of the 2D spatial manifold. The generalization in this paper for symmetry group $G_{f}=\mathbb{Z}_{2}^{f} \times_{\omega_{2}} G_{b}$ is that we put (at most) one of the $G_{b}$ species Majorana fermions into nontrivial pairings and all others vacuum pairings. If we consider the symmetry group $G_{b}=\mathbb{Z}_{2}^{T}$ and nontrivial 2-cocycle $\omega_{2}(T, T)=1$, our construction on a fixed triangular lattice reproduces the exactly solvable $T^{2}=-1$ topological superconductor model in Ref. [76].

To simplify our notations and make it easier to generalize to higher dimensions, we present some notations for Majorana fermion pairings. For two Majorana fermions $\gamma_{i, C}^{e}$ and $\gamma_{j, D}^{g^{-1} h}$ at vertices $i$ and $j\left(g, h \in G_{b} ; C, D \in\{A, B\}\right)$, we can choose the pairing such that

$$
-i \gamma_{i, C}^{e} \gamma_{j, D}^{g^{-1} h}=1,
$$

when acting on this state. We call it standard pairing, as the first Majorana fermion is labeled by the identity element $e \in G_{b}$. The standard pairing is illustrated in figures by a red arrow pointing from vertex $i C$ to vertex $j D$. For the nonstandard pairing between $\gamma_{i, C}^{g}$ and $\gamma_{j, D}^{h}$, we can use a $U(g)$ action on both sides of Eq. (96) and obtain

$$
-i \gamma_{i, C}^{g} \gamma_{j, D}^{h}=(-1)^{\omega_{2}\left(g, g^{-1} h\right)+s_{1}(g)\left(1+\delta_{C B}+\delta_{D B}\right)},
$$

where $\delta_{C B}=1(=0)$ if the Majorana fermion $\gamma_{i, C}^{\sigma}$ is the $B$ type (A-type) one. This difference comes from the symmetry transformations of $A$ - and $B$-type Majorana fermions [see Eqs. (94) and (95)]. For simplicity in describing the pairing, we introduce the projection operator of the Majorana fermion pairing as

$$
\begin{aligned}
P_{i C, j D}^{g, h} & :=U(g) P_{i C, j D}^{e, g^{-1} h} U(g)^{\dagger}=U(g) \frac{1}{2}\left(1-i \gamma_{i, C}^{e} \gamma_{j, D}^{g^{-1} h}\right) U(g)^{\dagger} \\
& =\frac{1}{2}\left[1-(-1)^{\omega_{2}\left(g, g^{-1} h\right)+s_{1}(g)\left(1+\delta_{C B}+\delta_{D B}\right)} i \gamma_{i, C}^{g} \gamma_{j, D}^{h}\right] .
\end{aligned}
$$

This generic pairing projection operator $P_{i C, j D}^{g, h}$ is obtained from a $U(g)$ action on the standard pairing projection 


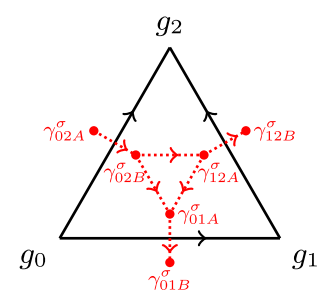

(a)

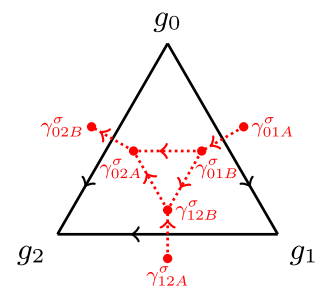

(b)
FIG. 2. Kasteleyn orientations of the resolved dual lattice. For a given triangulation of the 2D spatial spin manifold (shown by black links), we can construct a resolved dual lattice (shown by red links). The Majorana fermion pairings should respect the red link arrows in the figures.

operator $P_{i C, j D}^{e, g^{-1} h}=\left(1-i \gamma_{i, C}^{e} \gamma_{j, D}^{g^{-1} h}\right) / 2$. So the symmetric nature of the pairings can be easily seen from the symmetry transformations of the projection operators $\left(g, h, k \in G_{b}\right)$ :

$$
U(g) P_{i C, j D}^{h, k} U(g)^{\dagger}=P_{i C, j D}^{g h, g k}
$$

In $2 \mathrm{D}$ and higher dimensions, we use the generic pairing rule Eq. (97) and the projection operator Eq. (98) to construct $G_{b}$-symmetric states.

Decoration procedure.-Our Majorana fermions $\gamma_{i j, A}^{\sigma}$ and $\gamma_{i j, B}^{\sigma}\left(\sigma \in G_{b}\right)$ are on the two sides of each link $\langle i j\rangle$. We use the convention that the Majorana fermion on left-hand side (right-hand side) of the oriented link $\langle i j\rangle$ is $\gamma_{i j, A}^{\sigma}\left(\gamma_{i j, B}^{\sigma}\right)$. The vacuum pairing between them is from $A$ to $B$ : $-i \gamma_{i j, A}^{\sigma} \gamma_{i j, B}^{\sigma}=1$. To decorate Kitaev chains on the lattice, we should also add arrows to the small red triangle inside each triangle $\langle 012\rangle$ (see Fig. 2). These red arrows are constructed from the discrete spin structures (a choice of trivialization of Stiefel-Whitney homology class $w_{0}$ dual to cohomology class $w^{2}$ ) of the 2D spatial spin manifold triangulation. The Majorana fermions are designed to pair up with each other according to these red arrows. The red arrows constructed have the property that the number of counterclockwise arrows in a loop with even red links is always odd. This property is crucial for the decorated Kitaev chain to have fixed fermion parity. For details of the Kasteleyn orientations for arbitrary triangulation, we refer the interested readers to Ref. [53].

The Kitaev chain decoration is specified by $n_{1}\left(g_{i}, g_{j}\right) \in \mathbb{Z}_{2}$, which is a function of two group elements $g_{i}, g_{j} \in G_{b}$. If $n_{1}\left(g_{i}, g_{j}\right)=0$, the Majorana fermions $\gamma_{i j, A}^{\sigma}$ and $\gamma_{i j, B}^{\sigma}$ on the two sides of link $\langle i j\rangle$ are in vacuum pairings: $-i \gamma_{i j, A}^{\sigma} \gamma_{i j, B}^{\sigma}=1$ (for all $\sigma \in G_{b}$ ). On the other hand, if $n_{1}\left(g_{i}, g_{j}\right)=1$, there is a domain wall along the direction dual to link $\langle i j\rangle$, where a Kitaev chain is decorated [see the green belt shown in Eq. (100)]. For all $\left|G_{b}\right|$ species of Majorana fermions, we put only $\gamma_{i j, A}^{g_{i}}$ and $\gamma_{i j, B}^{g_{i}}$ to be in the nontrivial pairing. All other $\left|G_{b}\right|-1$ species of Majorana fermions $\gamma_{i j, A}^{\sigma}$ and $\gamma_{i j, B}^{\sigma}$ with $\sigma \neq g_{i}$ are still in vacuum pairings. Here is an example of the Kitaev chain decoration around the vertex $g_{2}$ inside a triangle (we omit the operator labels of Majorana fermions which are in vacuum pairings):

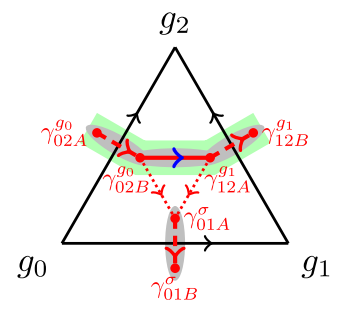

The domain wall decorated by a Kitaev chain is indicated by a green belt. Trivial (vacuum) pairings and nontrivial pairings are represented by dashed red lines and solid red lines, respectively. The red (blue) arrows show the trivial (nontrivial) pairing directions of Majorana fermions:

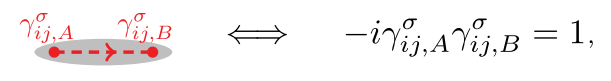

$$
\gamma_{02 B}^{g_{0}} \gamma^{\gamma_{12}^{g_{1}} A} \Longleftrightarrow-i \gamma_{02 B}^{g_{0}} \gamma_{12 A}^{g_{1}}=(-1)^{\omega_{2}\left(g_{0}, g_{0}^{-1} g_{1}\right)}
$$

We discuss more about the pairing directions and why they are symmetric later.

Consistency condition.-According to our decoration rule, the number of decorated Kitaev chains going through the boundary of a given triangle $\langle 012\rangle$ is

$$
\left(d n_{1}\right)\left(g_{0}, g_{1}, g_{2}\right)=n_{1}\left(g_{1}, g_{2}\right)+n_{1}\left(g_{0}, g_{2}\right)+n_{1}\left(g_{0}, g_{1}\right)
$$

Since we are constructing a gapped state without intrinsic topological order, there should be no dangling free Majorana fermions inside any triangle. Therefore, we have the $(\bmod 2)$ equation

$$
d n_{1}=0 .
$$

This equation is the consistency condition for the Kitaev chain decoration data $n_{1}$.

Symmetric pairing directions. - Now, let us turn back to the details of Majorana fermion pairings inside the triangle $\langle 012\rangle$. The strategy of constructing $G_{b}$-symmetric pairings is the same as in the 1D case: We first consider the standard triangle of $g_{0}=e$ and then apply a $U\left(g_{0}\right)$ action to obtain all other nonstandard triangles. The Majorana fermion pairings constructed in this way are automatically symmetric, due to the symmetry transformation rule of the pairing projection operators [Eq. (99)]. For the standard 
triangle, the Majorana fermions are paired (trivially or nontrivially) according to the Kasteleyn orientations indicated by red arrows. The pairings in the nonstandard triangle is obtained by a $U\left(g_{0}\right)$ action as follows:
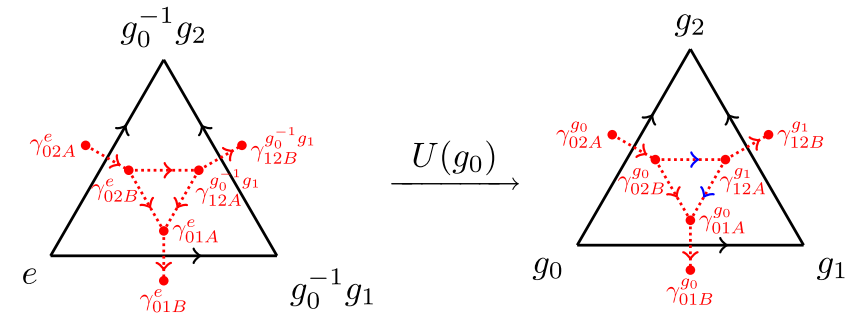

Note that Majorana fermions $\gamma_{i j, A}^{\sigma}$ and $\gamma_{i j, B}^{\sigma}\left(\sigma \neq g_{i}\right)$ of link $\langle i j\rangle$ are always in vacuum pairings $\left(-i \gamma_{i j, A}^{\sigma} \gamma_{i j, B}^{\sigma}=1\right)$, independent of the $n_{1}$ configurations. So their pairing directions always follow the red arrow Kasteleyn orientations in both figures of the above equation. For the two Majorana fermions $\gamma_{i j, A}^{g_{i}}$ and $\gamma_{i j, B}^{g_{i}}$ of the link $\langle i j\rangle$, there are two possibilities. If $n_{1}\left(g_{i}, g_{j}\right)=0$, these two Majorana fermions are also in vacuum pairing, with the direction indicated by the red arrow and projection operator

$P_{i j A, i j B}^{g_{i}, g_{i}}=U\left(g_{0}\right) P_{i j A, i j B}^{g_{0}^{-1} g_{i}, g_{0}^{-1} g_{i}} U\left(g_{0}\right)^{-1}=\frac{1}{2}\left(1-i \gamma_{i j, A}^{g_{i}} \gamma_{i j, B}^{g_{i}}\right)$.

On the other hand, if $n_{1}\left(g_{i}, g_{j}\right)=1$, we pair the Majorana fermion inside the triangle with another one belonging to another link with also $n_{1}=1$ [for example, $\gamma_{02 B}^{g_{0}}$ and $\gamma_{12 A}^{g_{1}}$ are paired in Eq. (100)]. Note that there are always an even number of Majorana fermions in nontrivial pairing among the three $\left(\gamma_{12 A}^{g_{1}}, \gamma_{02 B}^{g_{0}}\right.$, and $\left.\gamma_{01 A}^{g_{0}}\right)$ inside the triangle $\langle 012\rangle$, for we have $\left(d n_{1}\right)\left(g_{0}, g_{1}, g_{2}\right)=0(\bmod 2)$ from Eq. (104). There are three possible nontrivial pairings inside the triangle $\langle 012\rangle$, with Majorana pairing projection operators

$$
\begin{gathered}
P_{02 B, 01 A}^{g_{0}, g_{0}}=U\left(g_{0}\right) P_{02 B, 01 A}^{e, e} U\left(g_{0}\right)^{\dagger}=\frac{1}{2}\left(1-i \gamma_{02 B}^{g_{0}} \gamma_{01 A}^{g_{0}}\right), \\
P_{02 B, 12 A}^{g_{0}, g_{1}}=U\left(g_{0}\right) P_{02 B, 12 A}^{e, g_{0}^{-1} g_{1}} U\left(g_{0}\right)^{\dagger} \\
=\frac{1}{2}\left[1-(-1)^{\omega_{2}\left(g_{0}, g_{0}^{-1} g_{1}\right)} i \gamma_{02 B}^{g_{0}} \gamma_{12 A}^{g_{1}}\right], \\
P_{12 A, 01 A}^{g_{1}, g_{0}}=U\left(g_{0}\right) P_{12 A, 01 A}^{g_{0}^{-1} g_{1}, e} U\left(g_{0}\right)^{\dagger} \\
=\frac{1}{2}\left[1-(-1)^{\omega_{2}\left(g_{0}, g_{0}^{-1} g_{1}\right)+s_{1}\left(g_{0}\right)} i \gamma_{12 A}^{g_{1}} \gamma_{01 A}^{g_{0}}\right] .
\end{gathered}
$$

Among the three possible nontrivial pairings, only the last two may change their directions in the nonstandard triangle. They are indicated by blue arrows in the righthand-side figure in Eq. (105). This result can be understood from the following facts: The $(-1)^{\omega_{2}}$ term appears in the projection operators when the pairing is between Majorana fermions with different group element labels; and the $(-1)^{s_{1}}$ term appears when the pairing is between the same $A / B$-type Majorana fermions. The pairing Eq. (107) between $\gamma_{02 B}^{g_{0}}$ and $\gamma_{01 A}^{g_{0}}$ belongs to neither of the above two cases. So their pairing direction is the same as the red arrow even after $U\left(g_{0}\right)$ action.

Majorana fermion parity.-For convenience, we can define the Majorana fermion parity $P_{f}^{\gamma}(012)$ of a triangle $\langle 012\rangle$ to be the product of fermion parities of the Majorana fermion pairings along the three dashed red links inside the triangle. Since the symmetry action may change the pairing directions inside a triangle, the Majorana fermion parity of this triangle may also be changed. The fermion parity difference between the standard and nonstandard triangles can be calculated from the number of pairing arrows that are reversed by $U\left(g_{0}\right)$ action, which, of course, depends on the $n_{1}$ configurations. We can use, for example, $n_{1}\left(g_{0}, g_{1}\right) n_{1}\left(g_{1}, g_{2}\right)=0,1$ to indicate whether $\gamma_{12 A}^{g_{1}}$ and $\gamma_{01 A}^{g_{0}}$ are paired or not. So the Majorana fermion parity change inside the triangle is, in general, given by

$$
\begin{aligned}
\Delta P_{f}^{\gamma}(012) & =(-1)^{\omega_{2}\left(g_{0}, g_{0}^{-1} g_{1}\right)\left[n_{1}\left(g_{0}, g_{2}\right) n_{1}\left(g_{1}, g_{2}\right)+n_{1}\left(g_{0}, g_{1}\right) n_{1}\left(g_{1}, g_{2}\right)\right]+s_{1}\left(g_{0}\right) n_{1}\left(g_{0}, g_{1}\right) n_{1}\left(g_{1}, g_{2}\right)} \\
& =(-1)^{\omega_{2}\left(g_{0}, g_{0}^{-1} g_{1}\right) n_{1}\left(g_{1}, g_{2}\right)+s_{1}\left(g_{0}\right) n_{1}\left(g_{0}, g_{1}\right) n_{1}\left(g_{1}, g_{2}\right)} \\
& =(-1)^{\left(\omega_{2} \smile n_{1}+s_{1} \smile n_{1} \smile n_{1}\right)\left(g_{0}, g_{0}^{-1} g_{1}, g_{1}^{-1} g_{2}\right)},
\end{aligned}
$$

where we use $\left(d n_{1}\right)\left(g_{0}, g_{1}, g_{2}\right)=0$ from Eq. (104) in the second step. The above equation is a summary of phase factors from Eqs. (107)-(109). We note that the above expression is also true for negative oriented triangles. All the fermion parity change cases involve the particular Majorana fermion $\gamma_{12 A}^{g_{1}}\left(\gamma_{12 B}^{g_{1}}\right.$ for negative oriented triangles). We use it later in the definition of the $F$ symbol to compensate the fermion parity changes of the Majorana fermion pairing projection operators. 
Although the Majorana fermion parity of a given triangle may be changed, the fermion parity of the whole system is fixed under the global $U(g)$ action. Since the fermion parity of the vacuum pairings are not changed by symmetry actions, we need to consider only the $n_{1}$ domain walls decorated by Kitaev chains. For a particular (closed) Kitaev chain, the Majorana fermion parity is the same as the vacuum pairings if the pairings are constructed according to Kasteleyn orientations of the resolved dual lattice. It is also not hard to show that symmetry action always changes the arrow even times, following the pairing rules of Eq. (105). Therefore, we conclude all closed Kitaev chains have even fermion parity, although the local fermion parity of a triangle may be changed compare to the Kasteleyn orientations.

To sum up, among the $\left|G_{b}\right|$ species of Majorana fermions, we decorate exactly one Kitaev chain to each symmetry domain wall specified by the $n_{1}$ configurations of the state. The decoration is symmetric under symmetry actions. The Majorana fermion parity of a triangle is changed according to Eq. (110) compared to the Kasteleyn oriented pairings.

\section{Complex fermion decoration}

The rules of complex fermion decoration are much simpler than the pairings of Majorana fermions. The decoration is specified by a $\mathbb{Z}_{2}$-valued 2-cochain $n_{2} \in C^{1}\left(G_{b}, \mathbb{Z}_{2}\right)$. If $n_{2}\left(g_{i}, g_{j}, g_{k}\right)=0$, all the modes of complex fermions $c_{i j k}^{\sigma}\left(\sigma \in G_{b}\right)$ at the center of triangle $\langle i j k\rangle(i<j<k)$ are unoccupied. On the other hand, if $n_{2}\left(g_{i}, g_{j}, g_{k}\right)=1$, exactly one complex fermion mode $c_{i j k}^{g_{i}}$ is decorated at the center of triangle $\langle i j k\rangle$. All other complex fermions $c_{i j k}^{\sigma}\left(\sigma \neq g_{i}\right)$ are still in vacuum states.

The complex fermion decoration rule is $G_{b}$ symmetric. Under a $U(g)$ action, the vertex labels $\left\{g_{i}\right\}$ becomes $\left\{g g_{i}\right\}$. According to our decoration rule, the decorated complex fermion [if $n_{2}\left(g g_{i}, g g_{j}, g g_{k}\right)=n_{2}\left(g_{i}, g_{j}, g_{k}\right)=1$ ] should be $c_{i j k}^{g g_{i}}$, which is exactly the complex fermion $c_{i j k}^{g_{i}}$ by a $U(g)$ action.

\section{C. $F$ moves}

To compare the states on different triangulations of the 2D spatial manifold, we should consider the 2D Pachner move, which is essentially the retriangulation of a rectangle. The Pachner move induces a FSLU transformation of the FSPT wave functions from the right-hand-side triangulation lattice $\mathcal{T}^{\prime}$ to the left-hand-side lattice $\mathcal{T}$. We can first define the standard $F$ move for a rectangle with $g_{0}=e$; then other nonstandard ones can be obtained by simply a $U\left(g_{0}\right)$ action. The standard $F$ move is given by

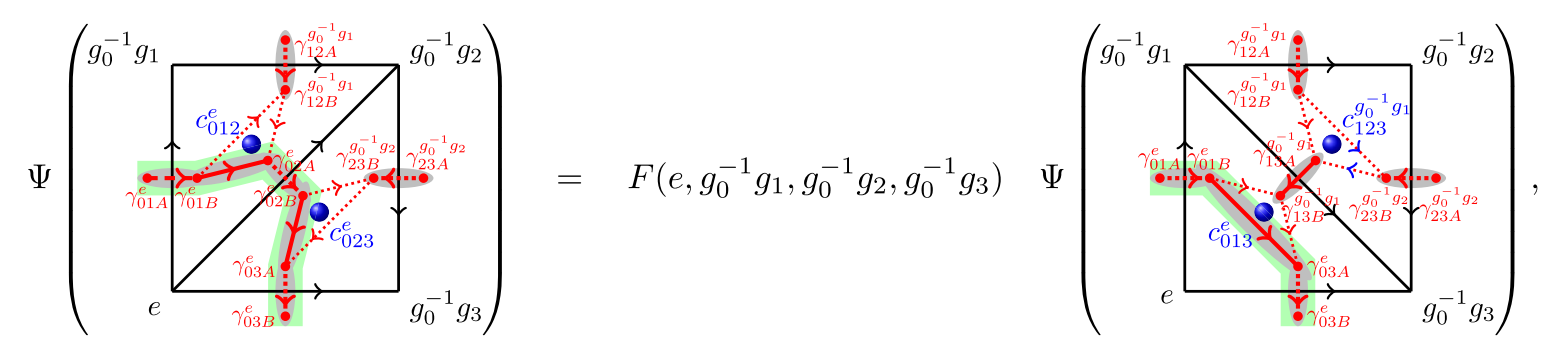

where the FSLU $F$ operator is defined as

$$
F(e, \overline{0} 1, \overline{0} 2, \overline{0} 3)=\nu_{3}(\overline{0} 1, \overline{1} 2, \overline{2} 3)\left(c_{012}^{e^{\dagger}}\right)^{n_{2}(012)}\left(c_{023}^{e^{\dagger}}\right)^{n_{2}(023)}\left(c_{013}^{e}\right)^{n_{2}(013)}\left(c_{123}^{g_{0}^{-1} g_{1}}\right)^{n_{2}(123)} X_{0123}\left[n_{1}\right]
$$

We use the abbreviation $\bar{i} j$ for $g_{i}^{-1} g_{j}$ in the arguments of $F$, and $n_{2}(i j k)$ represents $n_{2}\left(g_{i}, g_{j}, g_{k}\right)=n_{2}\left(g_{i}^{-1} g_{j}, g_{j}^{-1} g_{k}\right)$ for short.

The $U(1)$ phase factor $\nu_{3}(\overline{0} 1, \overline{1} 2, \overline{2} 3)=\nu_{3}\left(g_{0}^{-1} g_{1}\right.$, $\left.g_{1}^{-1} g_{2}, g_{2}^{-1} g_{3}\right)$ in the front of the $F$ symbol is an inhomogeneous 3-cochain depending on three group elements. By definition, it is related to the homogeneous cochain by

$\nu_{3}\left(g_{0}^{-1} g_{1}, g_{1}^{-1} g_{2}, g_{2}^{-1} g_{3}\right)=\nu_{3}\left(e, g_{0}^{-1} g_{1}, g_{0}^{-1} g_{2}, g_{0}^{-1} g_{3}\right)$, with the first argument of homogeneous cochain to be the identity element $e \in G_{b}$. Later, we use symmetry conditions to relate $\nu_{3}\left(e, g_{0}^{-1} g_{1}, g_{0}^{-1} g_{2}, g_{0}^{-1} g_{3}\right)$ and $\nu_{3}\left(g_{0}, g_{1}, g_{2}, g_{3}\right)$.

The complex fermion term of the form $c^{\dagger} c^{\dagger} c c$ annihilates two complex fermions at the two triangles of the righthand-side figure and creates two on the left-hand-side figure in Eq. (111). According to our decoration rules developed in Sec. V B 2, the triangle $\langle i j k\rangle$ is decorated by complex fermion $c_{i j k}^{g_{i}}$. So, in the standard $F$ move, only the last fermion $c_{123}^{g_{0}^{-1} g_{1}}$ has group element label $g_{0}^{-1} g_{1}$, and the 
other three fermions all have group element label $e$. We note that, different from the $G_{f}=\mathbb{Z}_{2}^{f} \times G_{b}$ case [53], the complex fermion parity of the 2D $F$ move does not need to be conserved, in general.

The term $X_{0123}\left[n_{1}\right]$ is related to the Kitaev chain decorations. In terms of Majorana fermion pairing projection operators [Eq. (98)], the general expression of the $X$ operator is

$$
\begin{aligned}
X_{0123}\left[n_{1}\right]=P_{0123}\left[n_{1}\right] \cdot\left(\gamma_{23 B}^{g_{0}^{-1} g_{2}}\right)^{d n_{2}(0123)} & \\
P_{0123}\left[n_{1}\right]= & \left(\prod_{\text {loopi }} 2^{\left(L_{i}-1\right) / 2}\right)\left(\prod_{\text {Majorana pairs }\langle a, b\rangle \text { in } \mathcal{T}} P_{a, b}^{g_{a}, g_{b}}\right) \\
& \times\left(\prod_{\operatorname{link}\langle i j\rangle \notin \mathcal{T}} \prod_{\sigma \in G_{b}} P_{i j A, i j B}^{\sigma, \sigma}\right) .
\end{aligned}
$$

The Majorana fermion $\gamma_{23 B}^{g_{0}^{-1} g_{2}}$ is added for fermion parity considerations (which are discussed in detail in the next section). If we do not add this term, $X_{0123}\left[n_{1}\right]$ projects the right-hand-side state to zero, whenever the Majorana fermion parity is changed under this $F$ move. We choose the Majorana fermion $\gamma_{23 B}^{g_{0}^{-1} g_{2}}$, because all the Majorana fermion parity change cases involve it in the standard $F$ move [see the blue arrows in Eq. (111)]. The pairing projection operator $P_{0123}\left[n_{1}\right]$ in Eq. (115) has three terms. The first term is a normalization factor, where $2 L_{i}$ is the length of the $i$ th loop in the transition graph of Majorana pairing dimer configurations on the left triangulation lattice $\mathcal{T}$ and right lattice $\mathcal{T}^{\prime}$. The second term projects the state to the Majorana pairing configuration state in the left figure. The third term is the product of the vacuum projection operators for those Majorana fermions that do not appear explicitly in the left figure. As an example, the explicit $X$ operator for the $n_{1}$ configurations shown in Eq. (111) is

$$
\begin{aligned}
X_{0123}\left[n_{1}\right]= & 2^{1 / 2}\left(P_{01 B, 02 A}^{e, e} P_{02 B, 03 A}^{e, e} \prod_{\sigma \neq e} P_{02 A, 02 B}^{\sigma, \sigma}\right) \\
& \times\left(\prod_{\sigma \in G_{b}} P_{13 A, 13 B}^{\sigma, \sigma}\right) .
\end{aligned}
$$

Since there are no pairings for the two blue arrow links in Eq. (111), the Majorana fermion parity is always conserved for this $n_{1}$ configuration.

The $F$ symbol constructed above should be a FSLU operator. So it should be both fermion parity even and symmetric under $G_{b}$ action. Similar to the $1 \mathrm{D}$ case, we can use these conditions to obtain several consistency equations for the cochains $n_{1}, n_{2}$, and $\nu_{3}$.

\section{Fermion parity conservation}

It is proved that the Majorana fermion parity is conserved under a 2D $F$ move if they are paired according to the Kasteleyn orientations in 2D [53]. Nevertheless, some of the links are not Kasteleyn oriented in the standard $F$ move Eq. (111), because the triangle $\langle 123\rangle$ is nonstandard; i.e., the group element label of the first vertex is not $e \in G_{b}$. It should be obtained from the standard one by a $U\left(g_{0}^{-1} g_{1}\right)$ action. So the blue arrows inside this triangle may change their directions according to our symmetric pairing rules. The Majorana fermion parity change of this triangle can be calculated from Eq. (110). Note that the three group element labels of the vertices are now $g_{0}^{-1} g_{1}, g_{0}^{-1} g_{2}$, and $g_{0}^{-1} g_{3}$. So the Majorana fermion parity change under the standard $F$ move is

$$
\Delta P_{f}^{\gamma}(F)=(-1)^{\left(\omega_{2} \smile n_{1}+s_{1} \smile n_{1} \smile n_{1}\right)\left(g_{0}^{-1} g_{1}, g_{1}^{-1} g_{2}, g_{2}^{-1} g_{3}\right)} .
$$

On the other hand, the complex fermion parity change under the $F$ move can be simply calculated by counting the complex fermion numbers of the two sides:

$$
\Delta P_{f}^{c}(F)=(-1)^{n_{2}\left(g_{0}^{-1} g_{1}, g_{1}^{-1} g_{2}\right)+n_{2}\left(g_{0}^{-1} g_{2}, g_{2}^{-1} g_{3}\right)+n_{2}\left(g_{0}^{-1} g_{1}, g_{1}^{-1} g_{3}\right)+n_{2}\left(g_{1}^{-1} g_{2}, g_{2}^{-1} g_{3}\right)}=(-1)^{d n_{2}\left(g_{0}^{-1} g_{1}, g_{1}^{-1} g_{2}, g_{2}^{-1} g_{3}\right)} .
$$

As a result, the conservation of total fermion parity $\Delta P_{f}=\Delta P_{f}^{\gamma} \cdot \Delta P_{f}^{c}=1$ under the $F$ move enforces the condition

$$
d n_{2}=\omega_{2} \smile n_{1}+s_{1} \smile n_{1} \smile n_{1} .
$$

It shows that the Majorana fermions and complex fermions are coupled to each other.
This result is very different from the 2D FSPT states with unitary group $G_{f}=\mathbb{Z}_{2}^{f} \times G_{b}$ (i.e., $\omega_{2}=0$ and $s_{1}=0$ ) [53], where the fermion parities of the Majorana fermions and complex fermions are conserved separately. So Eq. (119) is reduced to a simple cocycle equation $d n_{2}=0$. In the case of $T^{2}=-1$ topological superconductors [76], although both $\omega_{2}$ and $s_{1}$ are nontrivial, there combination $\omega_{2}+s_{1} \smile n_{1}=0$ is also trivial. So we still have $d n_{2}=0$. That is the reason why it admits the exactly solvable model with only Kitaev chain decorations. 


\section{Symmetry condition}

In the previous discussions, we consider only the standard $F$ move with $g_{0}=e$. The nonstandard $F$ moves are constructed by symmetry actions on the standard one. From Eq. (66), we have the following commuting diagram:

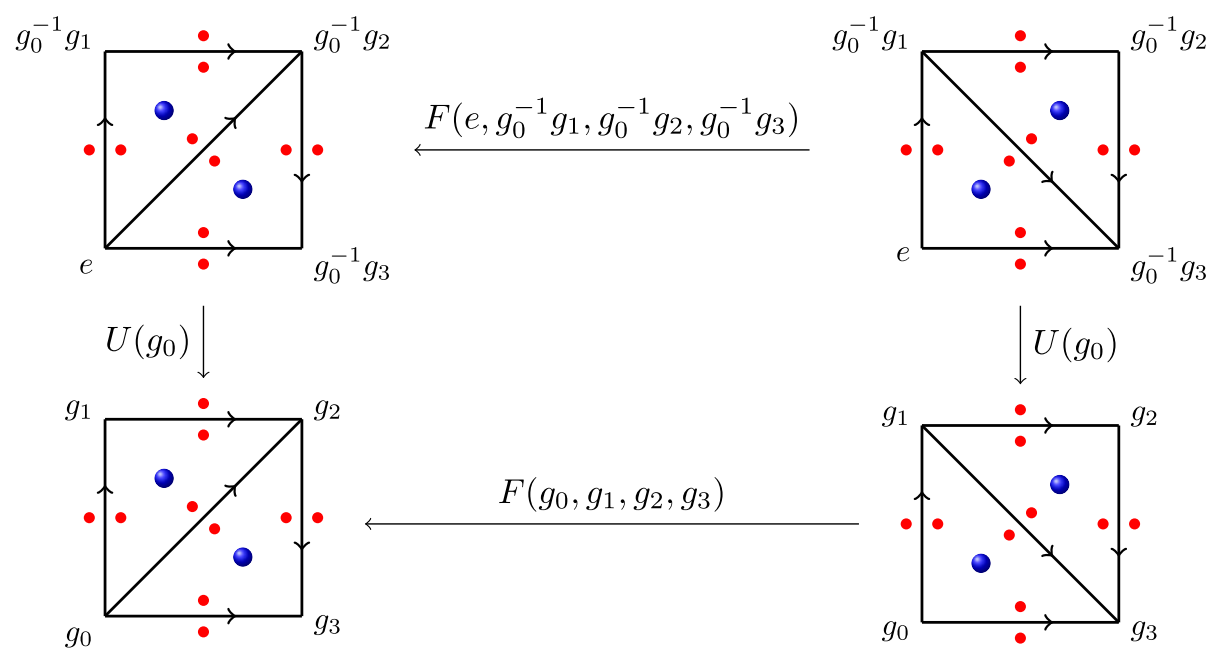

So the nonstandard $F$ operator is defined as

$$
F\left(g_{0}, g_{1}, g_{2}, g_{3}\right)=g_{0} F\left(e, g_{0}^{-1} g_{1}, g_{0}^{-1} g_{2}, g_{0}^{-1} g_{3}\right):=U\left(g_{0}\right) F\left(e, g_{0}^{-1} g_{1}, g_{0}^{-1} g_{2}, g_{0}^{-1} g_{3}\right) U\left(g_{0}\right)^{\dagger} \text {. }
$$

The $F$ moves constructed in this way are automatically symmetric, because we can derive the transformation rule

$$
F\left(g g_{0}, g g_{1}, g g_{2}, g g_{3}\right)=U(g) F\left(g_{0}, g_{1}, g_{2}, g_{3}\right) U(g)^{\dagger} \text {, }
$$

using Eq. (63) and the fact that $F$ is fermion parity even.

After a $U\left(g_{0}\right)$ action on the standard $F$ operator Eq. (112), we can obtain the generic $F$ symbol expression:

$$
F\left(g_{0}, g_{1}, g_{2}, g_{3}\right)=\nu_{3}\left(g_{0}, g_{1}, g_{2}, g_{3}\right)\left(c_{012}^{g_{0} \dagger}\right)^{n_{2}(012)}\left(c_{023}^{g_{0} \dagger}\right)^{n_{2}(023)}\left(c_{013}^{g_{0}}\right)^{n_{2}(013)}\left(c_{123}^{g_{1}}\right)^{n_{2}(123)} X_{0123}\left[n_{1}\right] \text {. }
$$

The complex fermions now have group element labels $g_{0}$ or $g_{1}$. And the $X$ operator is

$$
X_{0123}\left[n_{1}\right]=P_{0123}\left[n_{1}\right] \cdot\left(\gamma_{23 B}^{g_{2}}\right)^{d n_{2}(0123)}
$$

with added Majorana fermion $\gamma_{23 B}^{g_{2}}$ rather than $\gamma_{23 B}^{g_{0}^{-1} g_{2}} \cdot P_{0123}\left[n_{1}\right]$ has a similar expression as Eq. (115) that projects the Majorana fermions to the pairing state on the left-hand-side figure (the group element labels are changed appropriately).

From the decoration rules of Majorana fermions and complex fermions, $n_{1}$ and $n_{2}$ are invariant under symmetry actions. The generic homogeneous cochain $\nu_{3}$ in Eq. (123) is a combination of the inhomogeneous $\nu_{3}$ in the standard $F$ move and the \pm 1 signs appearing in the symmetry action. So we have the following symmetry conditions for $n_{1}, n_{2}$, and $\nu_{3}$ :

$$
\begin{gathered}
n_{1}\left(g_{0}, g_{1}\right)=n_{1}\left(e, g_{0}^{-1} g_{1}\right)=n_{1}\left(g_{0}^{-1} g_{1}\right) \\
n_{2}\left(g_{0}, g_{1}, g_{2}\right)=n_{2}\left(e, g_{0}^{-1} g_{1}, g_{0}^{-1} g_{2}\right)=n_{2}\left(g_{0}^{-1} g_{1}, g_{1}^{-1} g_{2}\right) \\
\nu_{3}\left(g_{0}, g_{1}, g_{2}, g_{3}\right)={ }^{g_{0}} \nu_{3}\left(g_{0}^{-1} g_{1}, g_{1}^{-1} g_{2}, g_{2}^{-1} g_{3}\right)=\nu_{3}\left(g_{0}^{-1} g_{1}, g_{1}^{-1} g_{2}, g_{2}^{-1} g_{3}\right)^{1-2 s_{1}\left(g_{0}\right)} \cdot \mathcal{O}_{4}^{\text {symm }}\left(g_{0}, g_{1}, g_{2}, g_{3}\right) .
\end{gathered}
$$

The symmetry sign $\mathcal{O}_{4}^{\text {symm }}$ in the last equation is given by 


$$
\begin{aligned}
\mathcal{O}_{4}^{\text {symm }}\left(g_{0}, g_{1}, g_{2}, g_{3}\right) & =(-1)^{\omega_{2}\left(g_{0}, g_{0}^{-1} g_{1}\right) n_{2}(123)+\left[s_{1}\left(g_{0}\right)+\omega_{2}\left(g_{0}, g_{0}^{-1} g_{2}\right)\right] d n_{2}(0123)} \\
& =(-1)^{\left(\omega_{2} \smile n_{2}+s_{1} \smile d n_{2}\right)\left(g_{0}, g_{0}^{-1} g_{1}, g_{1}^{-1} g_{2}, g_{2}^{-1} g_{3}\right)+\omega_{2}\left(g_{0}, g_{0}^{-1} g_{2}\right) d n_{2}\left(g_{0}^{-1} g_{1}, g_{1}^{-1} g_{2}, g_{2}^{-1} g_{3}\right)},
\end{aligned}
$$

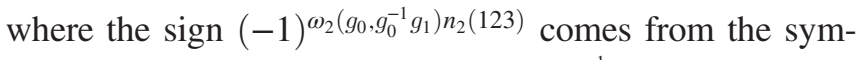
metry transformation Eq. (93) of $c_{123}^{g_{0}^{-1} g_{1}}$ and the sign $(-1)^{\left[s_{1}\left(g_{0}\right)+\omega_{2}\left(g_{0}, g_{0}^{-1} g_{2}\right)\right] d n_{2}(0123)}$ comes from the symmetry transformation Eq. (95) of $\gamma_{23 B}^{g_{0}^{-1} g_{2}}$ in the $X$ operator. We note that the last term $\omega_{2} d n_{2}$ in Eq. (128) is not a cup product or cup- 1 product form. This symmetry sign $\mathcal{O}_{4}^{\text {symm }}$ appears later in the twisted cocycle equation for $\nu_{3}$ as part of the obstruction function [see Eq. (131)].

\section{Superpentagon and twisted cocycle equations}

The $F$ moves should satisfy a consistency condition known as the pentagon equation for fusion categories. In a fermionic setting, it is a superpentagon equation with some fermion sign twist for superfusion categories [72,77,78]. The 2D FSPT states correspond to a special kind of superfusion category in which all the simple objects are invertible. So the classification of 2D FSPT states can be understood mathematically as the classification of pointed superfusion categories corresponding to a given symmetry group.

Similar to previous discussions, we need to consider only the standard superpentagon equation with $g_{0}=e$. All other superpentagon equations can be obtained from it by a $U\left(g_{0}\right)$ symmetry action. So it is enough to merely consider the standard superpentagon as coherence conditions. This standard superpentagon equation is shown in Fig. 3. Algebraically, we have the following equation:

$$
\begin{aligned}
F(e, \overline{0} 2, \overline{0} 3, \overline{0} 4) \cdot F(e, \overline{0} 1, \overline{0} 2, \overline{0} 4) & =F(e, \overline{0} 1, \overline{0} 2, \overline{0} 3) \cdot F(e, \overline{0} 1, \overline{0} 3, \overline{0} 4) \cdot F(\overline{0} 1, \overline{0} 2, \overline{0} 3, \overline{0} 4) \\
& =F(e, \overline{0} 1, \overline{0} 2, \overline{0} 3) \cdot F(e, \overline{0} 1, \overline{0} 3, \overline{0} 4) \cdot \overline{0} 1 F(e, \overline{1} 2, \overline{1} 3, \overline{1} 4)
\end{aligned}
$$

where we use $\bar{i} j$ to denote $g_{i}^{-1} g_{j}$. Note that only the last $F$ symbol is nonstandard in the above equation.

Now we can substitute the explicit expression of the standard $F$ move Eq. (112) into the standard superpentagon equation [Eq. (129)]. After eliminating all complex fermions and Majorana fermions, we obtain a twisted cocycle equation for the inhomogeneous 3-cochain $\nu_{3}$. In general, the twisted cocycle equation reads

$$
d \nu_{3}=\mathcal{O}_{4}\left[n_{2}\right]
$$

where $\mathcal{O}_{4}\left[n_{2}\right]$ is a functional of $n_{2}$ only (as well as $\omega_{2}$ and $s_{1}$ parametrizing the symmetry group, of course). The $n_{1}$ dependence of $\mathcal{O}_{4}$ is through $d n_{2}$ by Eq. (119). Since the fermion parities of Majorana fermions and complex fermions are coupled to each other, $\mathcal{O}_{4}\left[n_{2}\right]$ is much more complicated than the special result $\mathcal{O}_{4}\left[n_{2}\right]=(-1)^{n_{2}} \sim_{2}$ for unitary $G_{f}=\mathbb{Z}_{2}^{f} \times G_{b}[52,53,72]$.

From general considerations, the obstruction function $\mathcal{O}_{4}\left[n_{2}\right]$ consists of four terms:

$$
\mathcal{O}_{4}\left[n_{2}\right]=\mathcal{O}_{4}^{\text {symm }}\left[n_{2}\right] \cdot \mathcal{O}_{4}^{c}\left[n_{2}\right] \cdot \mathcal{O}_{4}^{c \gamma}\left[d n_{2}\right] \cdot \mathcal{O}_{4}^{\gamma}\left[d n_{2}\right] .
$$

These four terms have different physical meanings and are summarized as

$$
\begin{gathered}
\mathcal{O}_{4}^{\text {symm }}\left[n_{2}\right](01234) \\
=(-1)^{\left(\omega_{2} \smile n_{2}+s_{1} \smile d n_{2}\right)(01234)+\omega_{2}(013) d n_{2}(1234),} \\
\mathcal{O}_{4}^{c}\left[n_{2}\right]=(-1)^{n_{2} \smile n_{2}+d n_{2} \smile_{1} n_{2}}, \\
\mathcal{O}_{4}^{c \gamma}\left[d n_{2}\right]=(-1)^{d n_{2} \smile_{2} d n_{2}},
\end{gathered}
$$

$$
\begin{aligned}
\mathcal{O}_{4}^{\gamma} & {\left[d n_{2}\right](01234) } \\
& =(-1)^{d n_{2}(0124) d n_{2}(0234)}(-i)^{d n_{2}(0123)\left[1-d n_{2}(0124)\right]} \quad(\bmod 2)
\end{aligned}
$$

Note that the $d n_{2}$ terms in the exponent of $(-i)$ in the last equation should be understood as taking mod 2 values (can be only 0 or 1 ). And the notation $\smile_{i}$ is the higher cup product by Steenrod [65]. By adding a coboundary $(-1)^{d\left(s_{1} \smile n_{2}+n_{2} \smile_{2} d n_{2}\right)}$ to the obstruction function and shifting $\nu_{3} \rightarrow \nu_{3}(-1)^{s_{1} \smile n_{2}+n_{2} \smile_{2} d n_{2}}$, we can simplify the above obstruction function to 
$\mathcal{O}_{4}\left[n_{2}\right](01234)=(-1)^{\left(\omega_{2} \smile n_{2}+n_{2} \smile n_{2}+n_{2} \smile{ }_{1} d n_{2}\right)(01234)+\omega_{2}(013) d n_{2}(1234)+d n_{2}(0124) d n_{2}(0234)}(-i)^{d n_{2}(0123)\left[1-d n_{2}(0124)\right] \quad(\bmod 2)}$.

We note that only the first three terms $\omega_{2} \smile n_{2}+n_{2} \smile n_{2}+$ $n_{2} \smile_{1} d n_{2}$ in the exponent are expressed as a (higher) cup product form, while other terms are not. If we consider the special case of $\omega_{2}=0$ and $s_{1}=0$, then we have $d n_{2}=0$ from Eq. (119). So the above equation reduces to the known sign twist $\mathcal{O}_{4}\left[n_{2}\right]=(-1)^{n_{2} \smile n_{2}}$ in the superpentagon or supercohomology equation $[52,72]$.

Before calculating the obstruction function in detail, we note that we check numerically that the claimed expression Eq. (136) of $\mathcal{O}_{4}\left[n_{2}\right]$ is a cocycle, i.e., $d \mathcal{O}_{4}=1$, for arbitrary choices of $s_{1}, \omega_{2}, n_{1}$, and $n_{2}$ satisfying the corresponding consistency equations. This check is a consistency check, because the superpentagon equation [Eq. (129)] always implies a one-higher-dimensional equation involving one more vertex.

\section{Calculations of obstruction function $\mathcal{O}_{4}\left[n_{2}\right]$}

In this subsection, we give explicit calculations of the four terms of the obstruction function $\mathcal{O}_{4}\left[n_{2}\right]$ in Eq. (131).

The first term $\mathcal{O}_{4}^{\text {symm }}\left[n_{2}\right]$ comes from the $U(\overline{0} 1)$ symmetry action on $F(e, \overline{1} 2, \overline{1} 3, \overline{1} 4)$ in the last term in Eq. (129). The homogeneous $\nu_{3}$ in the nonstandard $F$ move is related to the inhomogeneous $\nu_{3}$ of the standard $F$ move by a symmetry action [see Eq. (127)]. So, using the explicit expression Eq. (128), we have

$$
\mathcal{O}_{4}^{\mathrm{symm}}\left[n_{2}\right](\overline{0} 1, \overline{0} 2, \overline{0} 3, \overline{0} 4)=(-1)^{\left(\omega_{2} \smile n_{2}+s_{1} \smile d n_{2}\right)(\overline{0} 1, \overline{1} 2, \overline{2} 3, \overline{3} 4)+\omega_{2}(\overline{0} 1, \overline{1} 3) d n_{2}(\overline{1} 2, \overline{2} 3, \overline{3} 4)},
$$

which is exactly Eq. (132) claimed above.

The second term $\mathcal{O}_{4}^{c}\left[n_{2}\right]$ is the fermion sign from reordering the complex fermion operators $\left(c_{i j k}^{g_{0}^{-1} g_{i}}\right)^{n_{2}\left(g_{i}, g_{j}, g_{k}\right)}$ in Eq. (129). To compare the complex fermion operators on the two sides of the superpentagon equation, one has to rearrange these operators and finally obtain the fermion sign

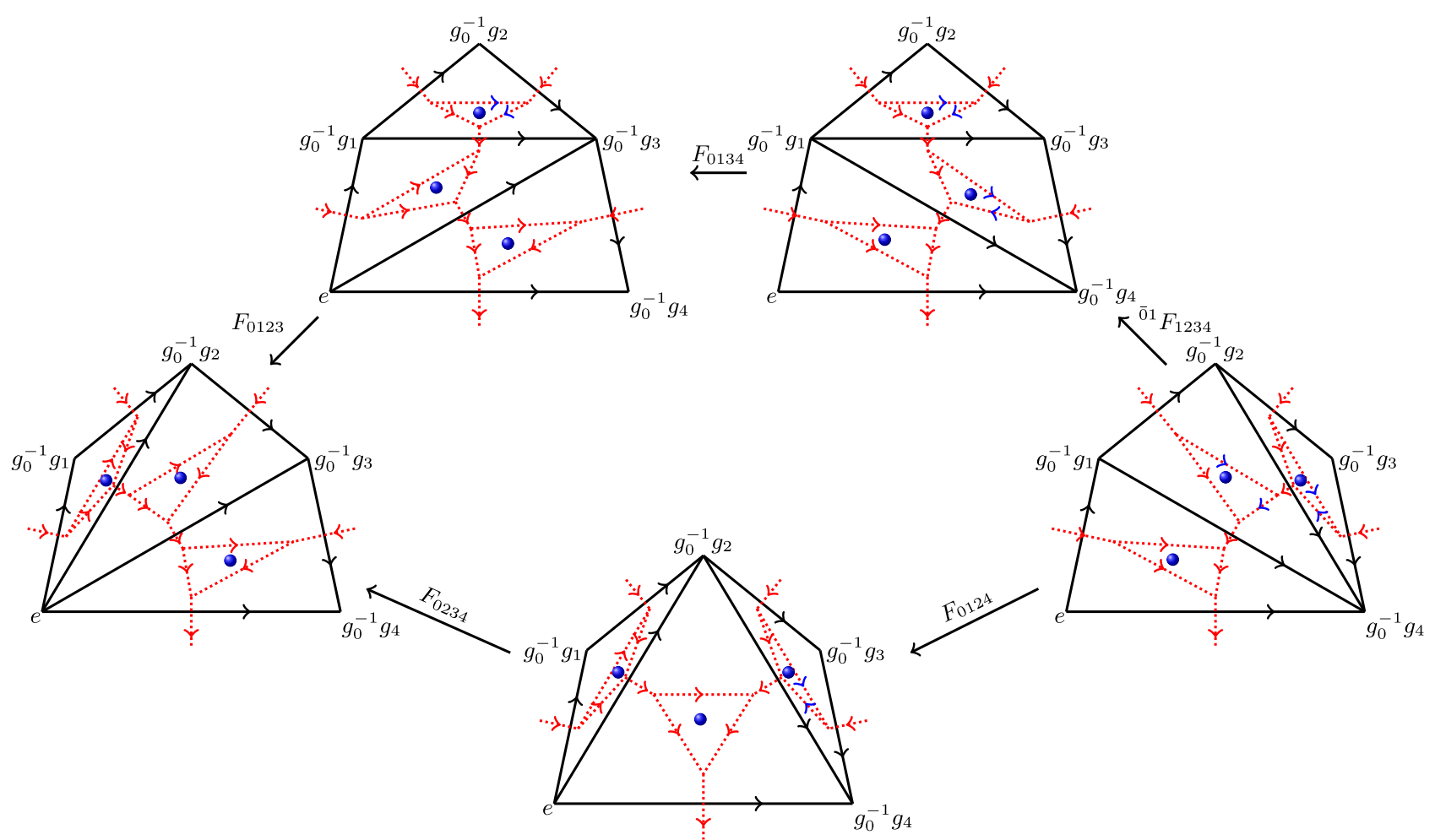

FIG. 3. Standard superpentagon equation. The dual trivalent graph of the triangulation is the usual string diagram pentagon equation for the tensor category. Algebraically, this standard superpentagon condition corresponds to Eq. (129). Since the group element label of the first vertex is $e \in G_{b}$, all the fermionic $F$ moves are standard except ${ }^{\overline{0} 1} F_{1234}$. Note that we use a simpler notation $F_{i j k l}=$ $F(e, \bar{i} j, \bar{j} k, \bar{k} l)$ in the figure. Blue arrows indicate that the Majorana fermion pairing directions may be changed compared to the red arrow Kasteleyn orientations. 


$$
\begin{aligned}
\mathcal{O}_{4}^{c}\left[n_{2}\right](01234) & =(-1)^{\left(n_{2} \smile n_{2}+d n_{2} \smile n_{2}\right)(01234)} \\
& =(-1)^{n_{2}(012) n_{2}(234)+\left[d n_{2}(0234) n_{2}(012)+d n_{2}(0134) n_{2}(123)+d n_{2}(0124) n_{2}(234)\right]} .
\end{aligned}
$$

This equation is a generalization of the usual sign twist $(-1)^{n_{2} \smile n_{2}}$ for 2-cocycle $n_{2}$. If $d n_{2} \neq 0$, we have another term $(-1)^{d n_{2} \smile_{1} n_{2}}$.

The third term $\mathcal{O}_{4}^{c \gamma}\left[d n_{2}\right]$ of the obstruction function originates from reordering the complex fermion and the Majorana fermions. For instance, to put all complex fermion operators to the front of Majorana fermion operators on the left-hand side of Eq. (129), we have to switch the $X$ operator of $F(0234)$ and the complex fermions of $F(0124)$. So there is a fermion sign $(-1)^{d n_{2}(0234) d n_{2}(0124)}$. Combining it with the fermion signs from the right-hand side, we have the total sign

$$
\begin{aligned}
\mathcal{O}_{4}^{c \gamma}\left[d n_{2}\right](01234)= & (-1)^{\left(d n_{2} \smile_{2} d n_{2}\right)(01234)} \\
& =(-1)^{d n_{2}(0123) d n_{2}(0134)+d n_{2}(0234) d n_{2}(0124)+d n_{2}(0123) d n_{2}(1234)+d n_{2}(0134) d n_{2}(1234)} .
\end{aligned}
$$

Since the fermion parities of the $X$ operator and the complex fermion operator $c^{\dagger} c^{\dagger} c c$ are related only to $d n_{2}$, this obstruction function $\mathcal{O}_{4}^{c \gamma}\left[d n_{2}\right]$ is a functional of $d n_{2}$ (rather than $n_{2}$ directly).

In the rest of this subsection, we calculate the most complicated part $\mathcal{O}_{4}^{\gamma}\left[d n_{2}\right]$ of the obstruction function. In addition to \pm 1 , this Majorana fermion term can also take values in $\pm i$. Whenever the Majorana fermion parity of the $F$ move is changed, i.e., $d n_{2}(0123)=1$, there is a dangling Majorana fermion $\gamma_{23 B}^{g_{0}^{-1} g_{2}}$ in the $X$ operator [Eq. (114)]. The presence of Majorana fermions depends only on $d n_{2}$. So, similar to $\mathcal{O}_{4}^{c \gamma}\left[d n_{2}\right]$, we expect $\mathcal{O}_{4}^{\gamma}\left[d n_{2}\right]$ to be a functional of $d n_{2}$ only.

We can denote the five $X$ operators in the standard superpentagon equation [Eq. (129)] by $X_{1234}=$
$P_{2}\left(\gamma_{34 B}^{\overline{0} 3}\right)^{d n_{2}(1234)}, \quad X_{0234}=P_{4}\left(\gamma_{34 B}^{\overline{0} 3}\right)^{d n_{2}(0234)}, \quad X_{0134}=$ $\left.P_{3}\left(\gamma_{34 B}^{\overline{0} 3}\right)\right)^{d n_{2}(0134)}, X_{0124}=P_{5}\left(\gamma_{24 B}^{\overline{0} 2}\right) d n_{2}(0124)$, and $X_{0123}=$ $P_{4}\left(\gamma_{23 B}^{\overline{0} 2}\right)^{d n_{2}(0123)}$. Here, $P_{i}$ is the Majorana pairing projection operator of the corresponding $i$ th figure $(1 \leq i \leq 5)$ in the superpentagon equation in Fig. 3. We use the convention that the rightmost figure is the first one with projection operator $P_{1}$, and the other four figures are counted counterclockwise. We also use the simpler notation

$$
\gamma_{i j B}^{\overline{0} i}:=\gamma_{i j B}^{g_{0}^{-1} g_{i}}
$$

Using these $X$ operators, the obstruction function coming from Majorana fermions can be calculated by

$$
\begin{aligned}
\mathcal{O}_{4}^{\gamma}\left[d n_{2}\right] & =\left\langle X_{1234}^{\dagger} X_{0134}^{\dagger} X_{0123}^{\dagger} X_{0234} X_{0124}\right\rangle \\
& =\left\langle P_{1}\left(\gamma_{34 B}^{\overline{0} 3}\right)^{d n_{2}(1234)} P_{2}\left(\gamma_{34 B}^{\overline{0} 3}\right)^{d n_{2}(0134)} P_{3}\left(\gamma_{23 B}^{\overline{0} 2}\right)^{d n_{2}(0123)} P_{4} P_{4}\left(\gamma_{34 B}^{\overline{0} 3}\right)^{d n_{2}(0234)} P_{5}\left(\gamma_{24 B}^{\overline{0} 2}\right)^{d n_{2}(0124)} P_{1}\right\rangle .
\end{aligned}
$$

The average is taken over the Majorana fermion state of the rightmost figure in Fig. 3. We also insert $P_{1}$, which is 1 acting on the rightmost state, at the first and the last places of the operator string.

Equation (140) should be calculated separately for different Majorana fermion configurations. Among the five dangling Majorana fermions of the five $X$ operators, only three of them are different:

$$
\left(\gamma_{34 B}^{\overline{0} 3}, \gamma_{24 B}^{\overline{0} 2}, \gamma_{23 B}^{\overline{0} 2}\right)
$$

So we can use the triple of their number,

$$
\left[d n_{2}(\hat{0})+d n_{2}(\hat{1})+d n_{2}(\hat{2}), d n_{2}(\hat{3}), d n_{2}(\hat{4})\right](\bmod 2),
$$

to indicate the presence or absence of the three Majorana fermions in Eq. (140). For simplicity, we use the notation

$$
d n_{2}(\hat{i}):=d n_{2}(01 \ldots \hat{i} \ldots 34),
$$

where $\hat{i}$ means that the number $i$ is removed from the argument. Each element of the triple corresponds to the Majorana fermion parity change of one or several $F$ moves. There are in total $2^{3} / 2=4$ different possibilities for the Majorana fermion parity changes (see the first column in Table IV), since the total Majorana parity of the five $F$ moves should be even. We can now calculate $\mathcal{O}_{4}^{\gamma}\left[d n_{2}\right]$ for these four cases separately.

As an example, let us calculate $\mathcal{O}_{4}^{\gamma}\left[d n_{2}\right]$ for the second case with Majorana fermion parity changes $(1,0,1)$ (the 
TABLE IV. Calculations of $\mathcal{O}_{4}^{\gamma}\left[d n_{2}\right]$ for all possible Kitaev chain configurations in the superpentagon equation in Fig. 3. The first column is the Majorana fermion parity change triple $\left[d n_{2}(\hat{0})+d n_{2}(\hat{1})+d n_{2}(\hat{2}), d n_{2}(\hat{3}), d n_{2}(\hat{4})\right](\bmod 2)$. There are in total four different cases. The second column is a simplified version of Eq. (140) for each case. The third and fourth columns are the Majorana pairing projection operators we use in calculation. The last column is the final result of $\mathcal{O}_{4}^{\gamma}\left[d n_{2}\right]$, which can be summarized by Eq. (152).

\begin{tabular}{|c|c|c|c|c|}
\hline$P_{f}^{\gamma}$ changes & Expression of $\mathcal{O}_{4}^{\gamma}\left[d n_{2}\right]$ & $P_{1}$ & $P_{4}$ & $\mathcal{O}_{4}^{\gamma}\left[d n_{2}\right]$ \\
\hline$\overline{(0,0,0)}$ & $\left\langle\left(\gamma_{34 B}^{03}\right) d n_{2}(\hat{0})+d n_{2}(\hat{2})+d n_{2}(\hat{1})\right\rangle$ & 1 & 1 & 1 \\
\hline$(1,0,1)$ & $\left\langle\left(\gamma_{34 B}^{\overline{0} 3}\right)^{d n_{2}(\hat{0})+d n_{2}(\hat{2})} \gamma_{23 B}^{\overline{0} 2}\left(\gamma_{34 B}^{\overline{0} 3}\right)^{d n_{2}(\hat{1})} P_{1}\right\rangle$ & $(-1)^{s_{1}(\overline{0} 2)+\omega_{2}(\overline{0} 2, \overline{2} 3)}\left(-i \gamma_{23 B}^{\overline{0} 2} \gamma_{34 B}^{\overline{0} 3}\right)$ & 1 & $-i$ \\
\hline$(1,1,0)$ & $\left\langle\left(\gamma_{34 B}^{\overline{0} 3}\right)^{d n_{2}(\hat{0})+d n_{2}(\hat{2})} P_{4}\left(\gamma_{34 B}^{\overline{0} 3}\right)^{d n_{2}(\hat{1})} \gamma_{24 B}^{\overline{0} 2} P_{1}\right\rangle$ & $(-1)^{\omega_{2}(\overline{0} 2, \overline{2} 3)}\left(-i \gamma_{34 B}^{\overline{0} 3} \gamma_{24 A}^{\overline{0} 2}\right)$ & $-i \gamma_{24 A}^{\overline{0} 2} \gamma_{24 B}^{\overline{0} 2}$ & $(-1)^{d n_{2}(\hat{1})}$ \\
\hline$(0,1,1)$ & $\left\langle\left(\gamma_{34 B}^{\overline{0} 3}\right)^{d n_{2}(\hat{0})+d n_{2}(\hat{2})} \gamma_{23 B}^{\overline{0} 2} P_{4}\left(\gamma_{34 B}^{\overline{0} 3}\right)^{d n_{2}(\hat{1})} \gamma_{24 B}^{\overline{0} 2} P_{1}\right\rangle$ & $-i \gamma_{23 B}^{\overline{0} 2} \gamma_{24 A}^{\overline{0} 2}$ & $-i \gamma_{24 A}^{\overline{0} 2} \gamma_{24 B}^{\overline{0} 2}$ & $(-1)^{d n_{2}(\hat{1})}$ \\
\hline
\end{tabular}

third row in Table IV). The dangling Majorana fermions present in Eq. (140) are $\gamma_{34 B}^{\overline{0} 3}$ and $\gamma_{23 B}^{\overline{0} 2}$. We can expand the projection operators $P_{i}$ to Majorana fermion operators. Since $\gamma_{34 B}^{\overline{0} 3}$ and $\gamma_{23 B}^{\overline{0} 2}$ are paired in the triangle $\langle 234\rangle$ of the rightmost figure in Fig. 3, we can consider only their pairing term [recall Eq. (98)]

$$
P_{23 B, 34 B}^{\overline{0} 2, \overline{0} 3}=\frac{1}{2}\left[1-(-1)^{\omega_{2}(\overline{0} 2, \overline{2} 3)+s_{1}(\overline{0} 2)} i \gamma_{23 B}^{\overline{0} 2} \gamma_{34 B}^{\overline{0} 3}\right]
$$

in $P_{1}$. All other projection operators $P_{i}$ with $i \neq 1$ can be chosen to be 1. So the obstruction function Eq. (98) can be calculated as

$$
\begin{aligned}
& \left.\mathcal{O}_{4}^{\gamma}\left[d n_{2}\right](01234)\right|_{(1,0,1)} \\
& =\left\langle\left(\gamma_{34 B}^{\overline{0} 3}\right) d n_{2}(\hat{0})+d n_{2}(\hat{2}) \gamma_{23 B}^{\overline{0} 2}\left(\gamma_{34 B}^{\overline{0} 3}\right)^{d n_{2}(\hat{1})} P_{1}\right\rangle \\
& =\left\langle\left(\gamma_{34 B}^{\overline{0} 3}\right) d n_{2}(\hat{0})+d n_{2}(\hat{2}) \gamma_{23 B}^{\overline{0} 2}\left(\gamma_{34 B}^{\overline{0} 3}\right)^{d n_{2}(\hat{1})}\right. \\
& \left.\times(-1)^{\omega_{2}(\overline{0} 2, \overline{2} 3)+s_{1}(\overline{0} 2)}\left(-i \gamma_{23 B}^{\overline{0} 2} \gamma_{34 B}^{\overline{0} 3}\right)\right\rangle
\end{aligned}
$$

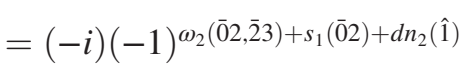

$$
\begin{aligned}
& =-i \text {. }
\end{aligned}
$$

Note that we replace $P_{1}$ by the second term of Eq. (144) to obtain Eq. (146) (see the third column in Table IV). In this way, the Majorana fermions all appear in Eq. (146) even times. After switching the Majorana fermions $\gamma_{23 B}^{\overline{0} 2}$ and $\left(\gamma_{34 B}^{\overline{0} 3}\right)^{d n_{2}(\hat{1})}$, we obtain a fermion sign $(-1)^{d n_{2}(\hat{1})}$. Then, we can eliminate all Majorana fermion operators, since their square is one. To simplify the phase factor Eq. (147), we observe that the conditions

$$
\begin{aligned}
& d n_{2}(\hat{3})=\left[\omega_{2}(\overline{0} 1, \overline{1} 2)+s_{1}(\overline{0} 1) n_{1}(\overline{1} 2)\right] n_{1}(\overline{2} 4)=0, \\
& d n_{2}(\hat{4})=\left[\omega_{2}(\overline{0} 1, \overline{1} 2)+s_{1}(\overline{0} 1) n_{1}(\overline{1} 2)\right] n_{1}(\overline{2} 3)=1
\end{aligned}
$$

imply $n_{1}(\overline{2} 3)=1$ and $n_{1}(\overline{2} 4)=0$. We also have $n_{1}(\overline{3} 4)=1$ from $d n_{1}(234)=0$. Using the relation

$$
\begin{aligned}
d n_{2}(\hat{1}) & =\omega_{2}(\overline{0} 2, \overline{2} 3) n_{1}(\overline{3} 4)+s_{1}(\overline{0} 2) n_{1}(\overline{2} 3) n_{1}(\overline{3} 4) \\
& =\omega_{2}(\overline{0} 2, \overline{2} 3)+s_{1}(\overline{0} 2)
\end{aligned}
$$

the exponent of $(-1)$ in Eq. (147) is, in fact, 0. We, therefore, have the final result $\left.\mathcal{O}_{4}^{\gamma}\left[d n_{2}\right](01234)\right|_{(1,0,1)}=-i$.

Similarly, we can calculate $\mathcal{O}_{4}^{\gamma}\left[d n_{2}\right]$ for all other cases of Majorana fermion parity changes. The information we need in the calculation is shown in Table IV. The final results shown in the last column in Table IV can be summarized into a simple expression (which is a functional of $d n_{2}$ only):

$$
\begin{aligned}
\mathcal{O}_{4}^{\gamma}\left[d n_{2}\right](01234)= & (-1)^{d n_{2}(\hat{3}) d n_{2}(\hat{1})}(-i)^{d n_{2}(\hat{4})\left[1-d n_{2}(\hat{3})\right]}(\bmod 2) \\
= & (-1)^{d n_{2}(0124) d n_{2}(0234)} \\
& \times(-i)^{d n_{2}(0123)\left[1-d n_{2}(0124)\right] \quad(\bmod 2)} . \quad(152)
\end{aligned}
$$

The $d n_{2}$ terms in the exponent of $(-i)$ should be understood as taking mod 2 values (can be only 0 or 1 ). This result is exactly the result claimed previously in Eq. (135).

\section{E. Boundary ASPT states in $\Gamma^{2}$}

We construct 2D FSPT states in the above discussions. However, not all of them correspond to distinct FSPT phases. In the following subsection, we construct explicitly a FSLU transformation path to connect an FSPT state with $n_{2}=\omega_{2}$ and a state without complex fermion decorations. The physical understanding is that there is a gapped symmetric boundary for the 2D FSPT state. So we conclude that the state with $n_{2}$, which is in the new coboundary subgroup

$$
\Gamma^{2}=\left\{\omega_{2} \in H^{2}\left(G_{b}, \mathbb{Z}_{2}\right)\right\},
$$

should be considered as in the trivial FSPT phase.

\section{FSLU to trivialize the 2D bulk}

Let us fix the symmetry group $G_{f}$ with given $G_{b}, \omega_{2}$, and $s_{1}$. We consider the special group supercohomology 2D FSPT state constructed from $\left(n_{2}, \nu_{3}\right)$ data satisfying $d n_{2}=0$ and $d \nu_{3}=\mathcal{O}_{4}=(-1)^{\omega_{2} \smile n_{2}+n_{2} \smile n_{2}}$ [52]. We show 


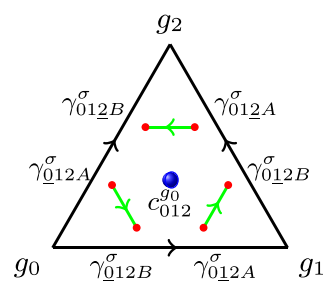

(a)

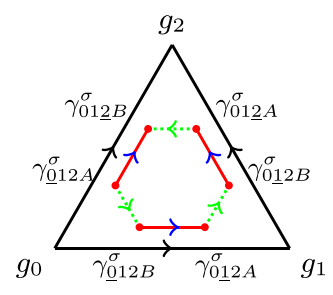

(b)

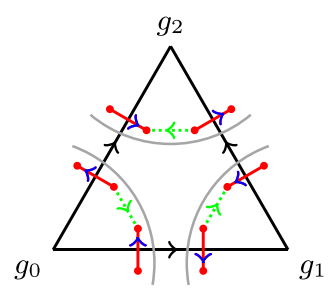

(c)

FIG. 4. FSLU transforms a 2D "FSPT" state to a fermion product state. There are two FSLU transformations $(a) \stackrel{U_{1}}{\rightarrow}(b) \stackrel{U_{2}}{\rightarrow}(c)$ to trivialize the initial 2D FSPT state. (a) Majorana fermions are in vacuum pairs (green arrows). (b) There is exactly one Kitaev Majorana chain inside the triangle (red links). (c) There is one Kitaev Majorana chain around each vertex (inside the gray arc). And we can shrink it to the vertex and redefine the basis state $\left|g_{i}\right\rangle$ for the vertex.

that the 2D FSPT state with $n_{2}=\omega_{2}$ can be connected to a product state by FSLU transformations.

Consider a 2D triangulation lattice of a closed oriented spatial manifold. The FSPT wave function is a superposition of the basis states with coefficients related to $\nu_{3}$ [see Eq. (90)]. The $n_{2}$ data specify the decorations of complex fermion $c_{i j k}^{g_{i}}$ at the center of each triangle $\langle i j k\rangle$ of the bosonic basis state $\left|\left\{g_{i}\right\}\right\rangle$. So the fermionic basis state can be expressed as

$$
|(a)\rangle=\prod_{\langle i j k\rangle}\left(c_{i j k}^{g_{i} \dagger}\right)^{n_{2}(i j k)}\left|\left\{g_{i}\right\}\right\rangle .
$$

We show in the following that the above state can be transformed by two FSLUs as

$$
|(a)\rangle \stackrel{U_{1}}{\rightarrow}|(b)\rangle \stackrel{U_{2}}{\rightarrow}|(c)\rangle
$$

where the schematic figures of these three states are shown in Fig. 4. The final state $|(c)\rangle$ is obtained from the bosonic state $\left|\left\{g_{i}\right\}\right\rangle$ by decorating a small Kitaev chain around each vertex [see the Kitaev chain along the gray arc in Fig. 4(c)]. After shrinking the small Kitaev chain to each vertex $i$, we can view the state $\left|g_{i}\right\rangle^{\prime}$ with a fermion mode as a new basis state. So the final state has the expression

$$
|(c)\rangle=\left|\left\{g_{i}\right\}\right\rangle^{\prime}=\bigotimes_{i}\left|g_{i}\right\rangle^{\prime}
$$

which is a fermionic product state without complex fermion decorations on the triangles. Therefore, using the two FSLUs $U_{1}$ and $U_{2}$, we have connect an FSPT state with complex fermion decorations specified by $n_{2}=\omega_{2}$ to another FSPT state without complex fermion decorations. So the decoration data $n_{2}=\omega_{2}$ for a complex fermion layer are trivialized.

The following are the detailed constructions for the two FSLU transformations.

(i) The first FSLU transformation $U_{1}$ from $|(a)\rangle$ to $|(b)\rangle$.- Apart from the complex fermion $c_{012}^{\sigma}$ (blue dots) at the center of the triangle in Fig. 4(a), we also add $3\left|G_{b}\right|$ fermion modes $\left(a_{\underline{012}}^{\sigma}, a_{0 \underline{12}}^{\sigma}\right.$, and $\left.a_{012}^{\sigma}\right)$ near the three vertices $(0,1$, and 2$)$ of the triangle and split them into $6\left|G_{b}\right|$ Majorana fermions [red dots in Fig. 4(a)]. The Majorana fermions are paired from $\gamma_{A}^{\sigma}$ to $\gamma_{B}^{\sigma}$ (vacuum pair) near each vertex, respecting the right-hand rule of the triangle orientation (green arrows). Since all the Majorana fermions are in vacuum pairings, we do not change the initial state Eq. (155) with only complex fermions $c_{i j k}^{g_{i}}$.

After the transformation by $U_{1}$, the initial state is changed to the intermediate state $|(b)\rangle$ shown in Fig. 4(b). In the state $|(b)\rangle$, there are exactly one nontrivial Majorana chain (red lines) and $\left|G_{b}\right|-1$ trivial Majorana chains (dotted green arrow) along the boundary of the triangle. The Majorana fermions forming nontrivial Kitaev chain are labeled by group elements of the nearby vertices. And the pairing directions are chosen to respect the $G_{b}$ symmetry. So the projection operators Eq. (98) for the nontrivial pairings (red lines) inside the triangle $\langle 012\rangle$ in Fig. 4(b) are

$$
\begin{aligned}
P_{\underline{012 B, 012 A}}^{g_{0}, g_{1}} & =U\left(g_{0}\right) P_{\underline{012 B, 012 A}}^{e, g_{0}^{-1} g_{1}} U\left(g_{0}\right)^{\dagger} \\
& =\frac{1}{2}\left[1-(-1)^{\omega_{2}\left(g_{0}, g_{0}^{-1} g_{1}\right)} i \gamma_{\underline{012 B}}^{g_{0}} \gamma_{0 \underline{12 A}}^{g_{1}}\right], \\
P_{0 \underline{012 B, 012 A}}^{g_{1}, g_{2}} & =U\left(g_{0}\right) P_{0 \underline{012 B, 012 A}}^{e, g^{-1} g_{2}} U\left(g_{0}\right)^{\dagger} \\
& =\frac{1}{2}\left[1-(-1)^{\omega_{2}\left(g_{1}, g_{1}^{-1} g_{2}\right)} i \gamma_{0 \underline{12 B}}^{g_{1}} \gamma_{01 \underline{2} A}^{g_{2}}\right], \\
P_{\underline{0} 12 A, 012 B}^{g_{0}, g_{2}} & =U\left(g_{0}\right) P_{\underline{012 A, 012 B}}^{e, g_{0}^{-1} g_{2}} U\left(g_{0}\right)^{\dagger} \\
& =\frac{1}{2}\left[1-(-1)^{\omega_{2}\left(g_{0}, g_{0}^{-1} g_{2}\right)} i \gamma_{\underline{012 A}}^{g_{0}} \gamma_{012 B}^{g_{2}}\right] .
\end{aligned}
$$

The blue arrow on the red link in Fig. 4(b) means that the actual arrow direction is obtained by a $G_{b}$ action and depends on $\omega_{2}$. The above Kitaev chain decoration procedure along the boundary of a triangle is very similar to the 1D FSPT construction with only three vertices. The Majorana pairings have the following properties: (i) Both the vacuum and the nontrivial pairings are invariant under $G_{b}$ action. (ii) The fermion parity change of Majorana chains from Fig. 4(a) to 
Fig. 4(b) inside the triangle is exactly $(-1)^{\omega_{2}\left(g_{0}^{-1} g_{1}, g_{1}^{-1} g_{2}\right)}$. This result is obtained by noticing that the summation of three $\omega_{2}$ terms in the above three equations is equal to $\omega_{2}\left(g_{0}^{-1} g_{1}, g_{1}^{-1} g_{2}\right)$, according to $d \omega_{2}\left(g_{0}, g_{0}^{-1} g_{1}, g_{1}^{-1} g_{2}\right)=0$. Since we choose $n_{2}=\omega_{2}$, we conclude that the complex fermion mode $c_{i j k}^{g_{i}}$ at the center of the triangle should be totally annihilated, to make sure that $U_{1}$ is fermion parity even.

In such a way, the FSLU transformation $U_{1}$ annihilates the fermions at the center of each triangle and creates a small Kitaev Majorana chain along the boundary of the triangle. The explicit expression of the standard FSLU transformation $U_{1}$ with $g_{0}=e$ is

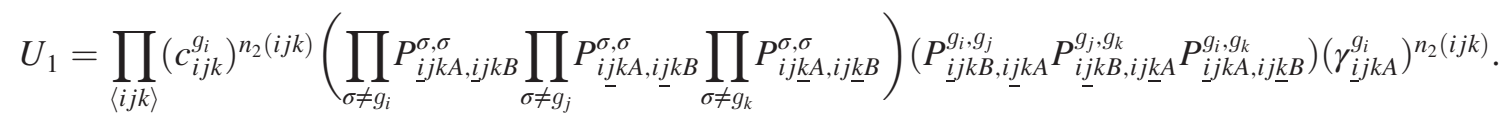

The first term $c_{i j k}^{g_{k}}$ is used to remove the complex fermions at the center of each triangle. Other terms are Majorana fermion pairing projection operators Eq. (98) to decorate one Kitaev chain along the boundary of the triangle. The last dangling Majorana operator is inserted for fermion parity considerations. Other nonstandard $U_{1}$ can be obtained from the standard one by a $U\left(g_{0}\right)$ action. Therefore, the operator $U_{1}$ from $|(a)\rangle$ to $|(b)\rangle$ is both fermion parity even and symmetric under $G_{b}$ action.

(ii) The second FSLU transformation $U_{2}$ from $|(b)\rangle$ to $|(c)\rangle$.- Since the state is on a closed oriented surface, there are four Majorana fermions on the two sides of the oriented link $\langle i j\rangle$. For convenience, we now relabel them by $\gamma_{i j 1}^{g_{i}}, \gamma_{i j 1}^{g_{j}}$ on the right-hand side and $\gamma_{i j 2}^{g_{i}}, \gamma_{i j 2}^{g_{j}}$ on the left-hand side of the oriented link $\langle i j\rangle$ (see the four red dots near each link). We can use an FSLU $U_{2}$ to change the Majorana fermion pairings from $P_{i j 1, i j 1}^{g_{i}, g_{j}}=P_{i j 2, i j 2}^{g_{i}, g_{j}}=1$ [see red links in Fig. 4(b)] to $P_{i j 1, i j 2}^{g_{i}, g_{i}^{-}}=P_{i j 2, i j 1}^{g_{j}, g_{j}^{-}}=1$ [see red links in Fig. 4(c)]. These four Majorana fermions near link $\langle i j\rangle$ form a loop with Kasteleyn orientations. So the Majorana fermion parity is unchanged under this FSLU.

The expression for the FSLU $U_{2}$ is simply

$$
U_{2}=\prod_{\langle i j\rangle} P_{\underline{i j 1, i j 2}}^{g_{i}, g_{i}} P_{i \underline{i j 2, i j 1}}^{g_{j}, g_{j}}
$$

Note that the actual direction of the blue arrow in Fig. 4(c) near vertex $i$ depends on $P_{i j 1, i j 2}^{g_{i}, g_{i}}=$ $U\left(g_{i}\right) \frac{1}{2}\left(1-i \gamma_{i j 1}^{e} \gamma_{i j 2}^{e}\right) U\left(g_{i}\right)^{\dagger}$. So the arrow direction is reversed if $s_{1}\left(g_{i}\right)=1$, and $\gamma_{i j 1}^{g_{i}}$ and $\gamma_{\underline{i j} 2}^{g_{i}}$ are Majorana fermions belonging to the same $A / B$ type.

After the above two FSLU transformations $U_{1}$ and $U_{2}$, we have a state where each vertex is surrounded by one nontrivial Majorana chain (red arrows) and $\left|G_{b}\right|-1$ trivial Majorana chains (green arrows). The fermion parity of this vertex depends on the number and the orientations of the triangles sharing this vertex. We can define a new state $\left|g_{i}\right\rangle^{\prime}$ around the vertex $i$ as the combination of the original bosonic state $\left|g_{i}\right\rangle$ and the neighboring Majorana fermions [the degrees of freedom inside the gray circles in Fig. 4(c)]. It is easy to check that $\left|g_{i}\right\rangle^{\prime}$ has the same $G_{b}$-transformation property as $\left|g_{i}\right\rangle$, i.e., $\left|g_{i}\right\rangle^{\prime} \rightarrow\left|g g_{i}\right\rangle^{\prime}$. So the final state $|(c)\rangle$ is a fermionic product state [Eq. (156)].

In summary, using the two FSLU transformations Eqs. (160) and (161), we can remove the complex fermions at the triangles of an FSPT state with $n_{2}=\omega_{2}$ and obtain an FSPT state with $n_{2}=0$. Therefore, the complex fermion decoration layer with $n_{2}=\omega_{2}$ is trivialized by these FSLU transformations.

\section{Boundary ASPT of the 2D bulk}

We show that the 2D FSPT state with $n_{2}=\omega_{2}$ on a closed surface can be connected to an FSPT state with $n_{2}=0$. However, for a system with a boundary, there is something unusual left.

Consider a state defined on a 2D triangulation lattice with a boundary. We can perform FSLU transformations similar to Eqs. (160) and (161). The only difference is that the link $\langle i j\rangle$ in the product in Eq. (161) is not on the boundary of the space manifold, since there are only two Majorana fermions near the each boundary link (see Fig. 5 for an example with only one interior vertex labeled by $g_{*}$ ). After the transformations, the bulk state becomes a tensor product of interior vertex state $\left|g_{i}\right\rangle^{\prime}$ [see Fig. 6(a) for an example]. But the boundary is transformed only under $U_{1}$ and is a so-called 1D ASPT state [see Fig. 6(b) for an example]. This boundary state is again a combination of one nontrivial Majorana chain [see red links in Fig. 6(b)] and $\left|G_{b}\right|-1$ trivial ones [see green links in Fig. 6(b)].

The anomalous feature of the boundary can be seen from the symmetry action on the boundary. Under a $U(g)$ symmetry action, the Majorana fermions are transformed as $\gamma_{A}^{g_{i}} \rightarrow(-1)^{\omega_{2}\left(g, g_{i}\right)} \gamma_{A}^{g g_{i}}$ and $\gamma_{B}^{g_{i}} \rightarrow(-1)^{\omega_{2}\left(g, g_{i}\right)+s_{1}(g)} \gamma_{B}^{g g_{i}}$. Since there are three types of Majorana pairings on the boundary [see Fig. 6(b)], we should analyze their symmetry transformations separately:

(i) Vacuum pairings (green lines) $-i \gamma_{A}^{g_{i}} \gamma_{B}^{g_{i}}=1$ are transformed trivially under $G_{b}$ action.

(ii) The nontrivial Majorana pairings (red lines) parallel to link $\langle i j\rangle$ are always $A-B$ type pairing with 


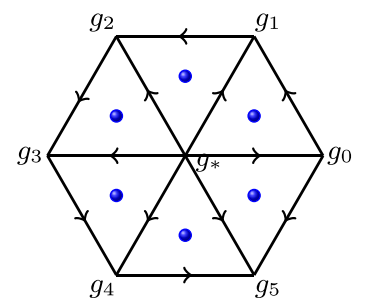

(a)

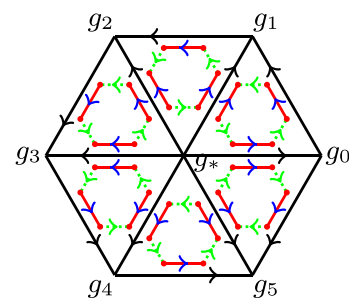

(b)

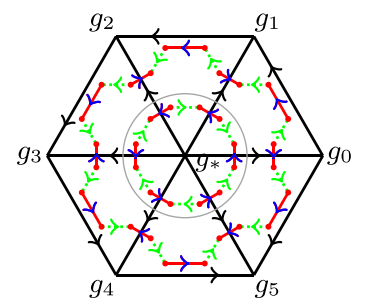

(c)

FIG. 5. FSLU transformations for "FSPT" state on a 2D surface with boundary. Similar to the "FSPT" state on a closed surface, there are two FSLU transformations $(a) \longrightarrow{ }^{U_{1}}(b) \longrightarrow{ }^{U_{2}}(c)$ to trivialize the initial 2D "FSPT" state. (a) Original 2D "FSPT" state with complex fermion decorations. (b) There is exactly one Kitaev's Majorana chain inside each triangle (red links). (c) There is one Kitaev's Majorana chain around each bulk vertex. The degrees of freedom inside the gray circle are combined to be the new basis state $\left|g_{*}\right\rangle^{\prime}$ for the bulk vertex. There is a remaining 1D ASPT state along the 1D boundary of the 2D bulk.

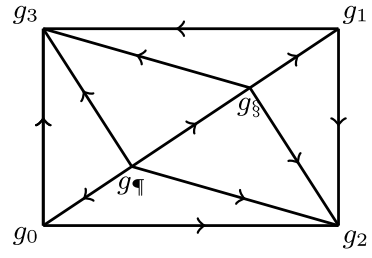

(a)

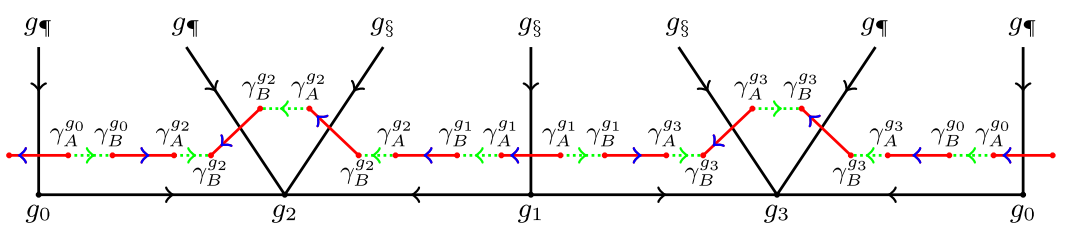

(b)

FIG. 6. ASPT on the 1D boundary of 2D bulk. On the boundary, the Majorana fermions forming a nontrivial Kitaev chain (red dots) are labeled by $A / B$ and $g \in G_{b}$. The blue arrow indicates that the pairing directions may be changed under symmetry action. (a) $2 \mathrm{D}$ bulk (b) $1 \mathrm{D}$ boundary.

different group element labels $g_{i}$ and $g_{j}$. Under the $g \in G_{b}$ action, the pairing arrow is changed according to $(-1)^{\omega_{2}\left(g, g_{i}\right)+\omega_{2}\left(g, g_{j}\right)}$.

(iii) The nontrivial Majorana pairings (red lines) crossing a black lattice link are always labeled by the same group element and can be of different $A / B$ types. So the pairing arrow is changed as $(-1)^{s_{1}(g)}$ if the pairing is $A-A$ or $B-B$ type.

Therefore, depending on $\omega_{2}\left(g, g_{i}\right), \omega_{2}\left(g, g_{j}\right)$, and $s_{1}(g)$, the local Majorana fermion parity (pairing direction) for the second and third types of pairings may be changed.

For a closed 1D array of Majorana fermions as the boundary of a 2D bulk [see Fig. 6(b), for example], it is not hard to show that the total Majorana fermion parity is always fixed under $G_{b}$ action [79]. However, if we want to define a similar state on an open chain [such as Fig. 6(b) with an open boundary condition], the total fermion parity may be violated under $U(g)$ action. This result is simply because the direction of the link crossing the boundary may be changed. So the symmetry action is incompatible with the fermion parity of the open 1D ASPT chain. It implies that the 1D ASPT state can exist only on the boundary of a 2D bulk state.

\section{Boundary $F$ move and fermion parity violation}

There is another way to understand the anomalous feature of the $1 \mathrm{D}$ boundary. We can try to construct such a 1D state without 2D bulk directly and find out the inconsistency of the state.

We consider the 1D state with a Kitaev chain and without complex fermion decoration $\left(n_{1}=0\right)$. We put $\left|G_{b}\right|$ species of Majorana fermions near each vertex similar to the 2D construction. But only one of them is in nontrivial pairings between different vertices. The $F$ move for this state is given by

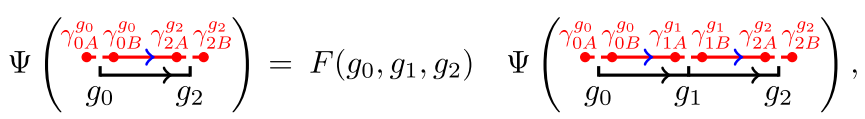

where the FSLU $F$ operator is defined as

$F\left(g_{0}, g_{1}, g_{2}\right)=\left|G_{b}\right|^{1 / 2} \nu_{2}\left(g_{0}, g_{1}, g_{2}\right) X($ violate fermion parity $)$.

$X$ is some Majorana fermion projection operators to impose the Majorana pairings. Since the Majorana fermion parity change of the $F$ move is $(-1)^{\omega_{2}\left(g_{0}^{-1} g_{1}, g_{1}^{-1} g_{2}\right)}$ (one can check directly using Kasteleyn orientations), the above $F$ symbol may violate the Majorana fermion parity. So the state is obstructed if $\left[\omega_{2}\right]$ is nontrivial.

However, we can introduce a $2 \mathrm{D}$ bulk to the above 1D state we are constructing. The difference is that we can use 


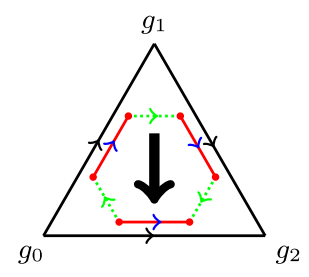

(a)

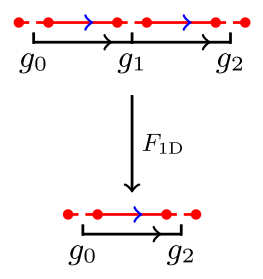

(b)
FIG. 7. Relation between FSLU $U_{1}$ for a $2 \mathrm{D}$ triangle and $1 \mathrm{D} F$ move. The $2 \mathrm{D}$ triangle can be viewed as a 1D $F$ move from the upper two links to the lower link. It relates the trivialization of 2D $n_{2}$ data to the obstruction of a 1D Kitaev chain. (a) The FSLU transformation $U_{1}$ in Eq. (160) for a single triangle. This FSLU changes the fermion parities of the Majorana fermions and the complex fermions by $(-1)^{\omega_{2}(\overline{0} 1, \overline{2} 3)}$ and $(-1)^{n_{2}(012)}$, respectively. (b) The 1D $F$ move Eq. (164) for the boundary ASPT state. This $F$ move changes the fermion parities of the Majorana fermions and the complex fermions by $(-1)^{\omega_{2}(\overline{0} 1, \overline{2} 3)}$ and $(-1)^{n_{2}(012)}$, respectively.

a complex fermion from the 2D bulk to compensate the Majorana fermion parity of the $1 \mathrm{D} F$ move Eq. (163). So the new $F$ move reads

$$
F\left(g_{0}, g_{1}, g_{2}\right)=\left|G_{b}\right|^{1 / 2} \nu_{2}\left(g_{0}, g_{1}, g_{2}\right)\left(c_{012}^{g_{0}}\right)^{n_{2}\left(g_{0}, g_{1}, g_{2}\right)} X
$$

We have to impose the condition

$$
n_{2}=\omega_{2}
$$

to make the new $F$ move total fermion parity even. So there is no longer fermion parity inconsistency for the 1D ASPT state on the boundary of a $2 \mathrm{D}$ bulk.

In fact, the 1D $F$ move Eqs. (162) and (164) can be understood as the FSLU transformation $U_{1}$ Eq. (160) for a single triangle $\langle 012\rangle$ (see Fig. 7). The upper two links $\langle 01\rangle$ and $\langle 12\rangle$ correspond to the right-hand side of the $1 \mathrm{D} F$ move. And the lower link $\langle 02\rangle$ corresponds to the left-hand side of the 1D $F$ move. The additional complex fermion $c_{012}^{g_{0}}$ in $1 \mathrm{D} F$ move Eq. (164) is merely the decorate complex fermion at the center of the 2D triangle. This picture relates the 1D FSPT obstruction and the 2D FSPT trivialization and explains the trivialization relation Eq. (165) in a simple way.

\section{F. Classification of 2D FSPT phases}

The general classification of 2D FSPT phases is as follows. We first calculate the cohomology groups $H^{1}\left(G_{b}, \mathbb{Z}_{2}\right), H^{2}\left(G_{b}, \mathbb{Z}_{2}\right)$, and $H^{3}\left[G_{b}, U(1)_{T}\right]$. For each $n_{1} \in H^{1}\left(G_{b}, \mathbb{Z}_{2}\right)$, we solve the twisted cocycle equation [Eq. (14)] for $n_{2}$. For each solution $n_{2}$, we solve the twisted cocycle equation [Eq. (14)] for $\nu_{3}$. If $n_{2}$ and $\nu_{3}$ are in the trivialization subgroup $\Gamma^{2}$ and $\Gamma^{3}$ in Eq. (15), then they are trivialized by boundary ASPT states. (Similar to the 1D case, we can define $\Gamma^{3}$ to describe those 2D BSPT state trivialized by fermions.) So the obstruction-free and trivialization-free $\left(n_{1}, n_{2}, \nu_{3}\right)$ fully classify the 2D FSPT phases.

Similar to the bosonic case, we can also use the 2D FSLU transformations to construct the commuting-projector parent Hamiltonians. The procedure is tedious but straightforward. For the case of complex fermion decorations only, it is given explicitly in Ref. [72]. The terms of the Hamiltonian are sequences of fermionic $F$ moves that change the group element label of a vertex from one to another. All the terms commute with each other, because our FSPT wave function is at the fixed point.

\section{FIXED-POINT WAVE FUNCTION AND CLASSIFICATION OF FSPT STATES IN 3D}

The fixed-point wave function for a FSPT state in 3D has four layers of degrees of freedom. It is a superposition of all possible basis states as (we omit the 2D $p+i p$ chiral superconductor layer in the figure for simplicity)

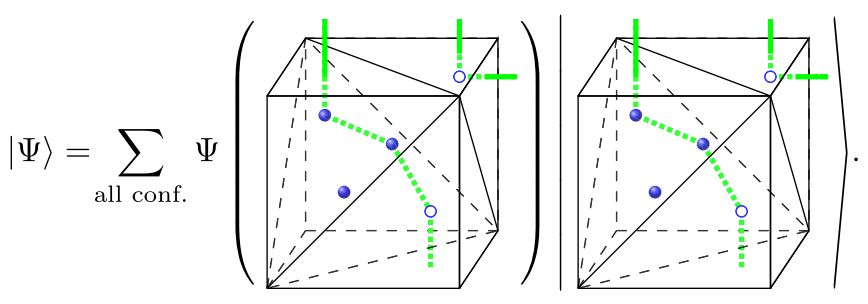

The basis state is labeled by group elements of $G_{b}$ on each vertex. On the plane dual to each link, we put a $2 \mathrm{D} p+i p$ chiral superconductor. Different from other layers, the $p+$ ip superconductor layer does not have a fixed-point wave function on a discrete lattice [80]. So we do not discuss this layer decoration until the end of this section. Along the dual link of each triangle, we decorate Kitaev chains (see green lines in the above equation). And at the center of each tetrahedron, we decorate some complex fermions (see blue dots in the above equation).

\section{A. Four layers of degrees of freedom}

Similar to 2D, we construct FSPT states on a 3D lattice by decorating complex fermions, Kitaev chains, and $p+i p$ superconductors to the BSPT states. Therefore, there are four layers of degrees of freedom including the bosonic ones on a $3 \mathrm{D}$ triangulation lattice:

(i) $\left|G_{b}\right|$ level bosonic (spin) state $\left|g_{i}\right\rangle\left(g_{i} \in G_{b}\right)$ on each vertex $i$,

(ii) $\left|G_{b}\right|$ species of complex fermions $c_{i j k l}^{\sigma}\left(\sigma \in G_{b}\right)$ at the center of each tetrahedron $\langle i j k l\rangle$,

(iii) $\left|G_{b}\right|$ species of complex fermions (split to Majorana fermions) $a_{i j k}^{\sigma}=\left(\gamma_{i j k, A}^{\sigma}+i \gamma_{i j k, B}^{\sigma}\right) / 2\left(\sigma \in G_{b}\right)$ on the two sides of each triangle $\langle i j k\rangle$, and 


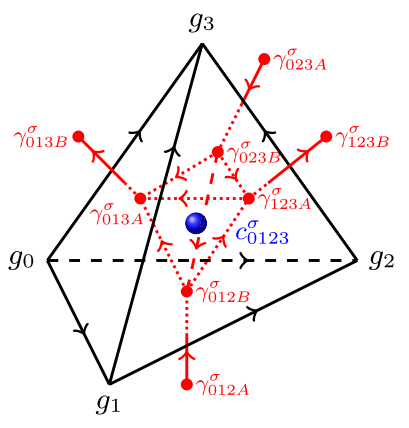

(a)

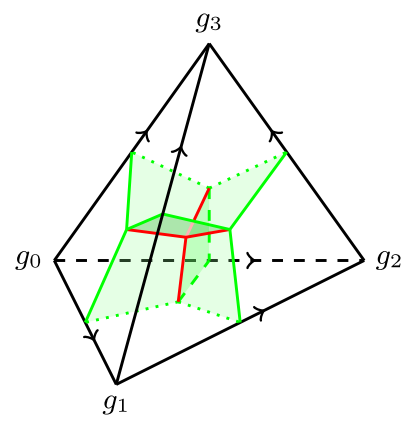

(b)
FIG. 8. Four layers of degrees of freedom in a tetrahedron of a 3D triangulation lattice. (a) Layers of bosonic state $\left|g_{i}\right\rangle$ on each vertex $i$, complex fermions $c_{i j k l}^{\sigma}$ at the center of each tetrahedron $\langle i j k l\rangle$, and Majorana fermions $\gamma_{i j k, A / B}^{\sigma}$ on the two sides of each triangle $\langle i j k\rangle$. (b) Layer of a 2D $p+i p$ chiral superconductor on the (green) plane dual to each link $\langle i j\rangle$. The boundary chiral Majorana modes of the $p+i p$ superconductors are along the (red) intersecting lines of the (green) planes.

(iv) $\left|G_{b}\right|$ species of $2 \mathrm{D} p+i p$ chiral superconductors [may have several copies indicated by $\left.n_{1}\left(g_{i}, g_{j}\right) \in \mathbb{Z}_{T}\right]$ on the plane dual to link $\langle i j\rangle$. The boundary chiral Majorana modes (along the link dual to some triangle) are $\psi_{i j, L ; \alpha}^{\sigma}$ or $\psi_{i j, R ; \alpha}^{\sigma}$ ( $\left.\sigma \in G_{b}\right)$ depending on the chirality (left- or righthand rule with respect to the oriented link $\langle i j\rangle)$. Here, $\alpha$ labels the number of the chiral Majorana modes $\left[\alpha=1,2, \ldots,\left|n_{1}\left(g_{i}, g_{j}\right)\right|\right]$.

The four layers of degrees of freedom are summarized in one tetrahedron of the 3D triangulation lattice in Fig. 8. The four vertices of the tetrahedron are labeled by $g_{0}, g_{1}, g_{2}, g_{3} \in G_{b}$. In Fig. 8(a), the blue ball is the complex fermion $c_{0123}^{\sigma}\left(\sigma \in G_{b}\right)$ at the center of the tetrahedron. Red dots represent Majorana fermions $\gamma_{i j k, A}^{\sigma}$ and $\gamma_{i j k, B}^{\sigma}\left(\sigma \in G_{b}\right)$ on the two sides of the triangle $\langle i j k\rangle$. We use the convention that the direction pointing from $\gamma_{i j k, A}^{\sigma}$ to $\gamma_{i j k, B}^{\sigma}$ is the same as the right-hand rule orientation of the triangle $\langle i j k\rangle$. In Fig. 8 (b), each green area dual to link $\langle i j\rangle$ represents the decorated 2D $p+i p$ superconductors. There are $\left|n_{1}\left(g_{i}, g_{j}\right)\right|$ right-moving or left-moving chiral Majorana modes $\psi_{i, R / L ; \alpha}^{g_{i}}\left(\alpha=1,2, \ldots,\left|n_{1}\right|\right)$ along the boundary (red line) of the green area dual to each link $\langle i j\rangle$.

As discussed in Sec. III B, the symmetry transformation rules of these degrees of freedom under $G_{b}$ are summarized as follows $\left(g, g_{i}, \sigma \in G_{b}\right)$ :

$$
\begin{gathered}
U(g)\left|g_{i}\right\rangle=\left|g g_{i}\right\rangle, \\
U(g) c_{i j k l}^{\sigma} U(g)^{\dagger}=(-1)^{\omega_{2}(g, \sigma)} c_{i j k l}^{g \sigma}, \\
U(g) \gamma_{i j k, A}^{\sigma} U(g)^{\dagger}=(-1)^{\omega_{2}(g, \sigma)} \gamma_{i j k, A}^{g \sigma},
\end{gathered}
$$

$$
\begin{array}{r}
U(g) \gamma_{i j k, B}^{\sigma} U(g)^{\dagger}=(-1)^{\omega_{2}(g, \sigma)+s_{1}(g)} \gamma_{i j k, B}^{g \sigma}, \\
U(g) \psi_{i j, R ; \alpha}^{\sigma} U(g)^{\dagger}=(-1)^{\omega_{2}(g, \sigma)} \psi_{i j, g(R) ; \alpha}^{g \sigma}, \\
U(g) \psi_{i j, L ; \alpha}^{\sigma} U(g)^{\dagger}=(-1)^{\omega_{2}(g, \sigma)+s_{1}(g)} \psi_{i j, g(L) ; \alpha}^{g \sigma} .
\end{array}
$$

The bosonic degrees of freedom always form a linear representation of $G_{b}$ (and $G_{f}$ ). On the other hand, the fermion modes support projective representations of $G_{b}$ with coefficient $(-1)^{\omega_{2}}$ and, hence, linear representations of $G_{f}$ by Eq. (64).

\section{B. Decorations of fermion layers}

In this section, we construct systematic procedures of decorating Kitaev chains and complex fermions to the bosonic basis state $\left|\left\{g_{i}\right\}\right\rangle$. Each layer of the degrees of freedom twists the consistent equations for the next layer. The decoration rules should respect the symmetry in all layers.

We focus on the Kitaev chain and complex fermion decoration here, and the decorations of $2 \mathrm{D} p+i p$ superconductors are discussed at the end of this section.

\section{Kitaev chain decoration}

The Kitaev chain decoration in 3D is similar to the constructions in Ref. [53]. The difference is that we put $\left|G_{b}\right|$ species of Majorana fermions rather than one. However, we still put only one species of Majorana fermions into nontrivial pairings along the decorated Kitaev chain.

a. Decoration procedure.-For a given 3D triangulation lattice, we first construct the resolved dual lattice (red lattice shown in Fig. 9). Our Majorana fermions $\gamma_{i j k, A}^{\sigma}$ and $\gamma_{i j k, B}^{\sigma}\left(\sigma \in G_{b}\right)$ are at the (red) vertices on the two sides of each (black) triangle $\langle i j k\rangle$. The red arrow follows the convention that the direction from $\gamma_{i j k, A}^{\sigma}$ to $\gamma_{i j k, B}^{\sigma}$ is the same as the right-hand rule orientation of the triangle $\langle i j k\rangle$. The direction of vacuum pairing between them is from $A$ to $B$ : $-i \gamma_{i j k, A}^{\sigma} \gamma_{i j k, B}^{\sigma}=1$ when acting on the state. To decorate Kitaev chains on the red lattice, we also should add arrows to the small red tetrahedron inside each black tetrahedron (see Fig. 9). These red arrows are constructed from the discrete spin structures (a choice of trivialization of StiefelWhitney homology class $w_{1}$ dual to cohomology class $w^{2}$ ) of the 3D spatial spin manifold triangulation. The Majorana fermions are always paired according to these red arrows on the red lattice. The red arrows have the property that the number of counterclockwise arrows in the smallest red loop around each black link is always odd. For details of the local Kasteleyn orientations for arbitrary triangulation, we refer the interested reader to Ref. [53].

The Kitaev chain decoration on the red lattice is specified by $n_{2}\left(g_{i}, g_{j}, g_{k}\right) \in \mathbb{Z}_{2}$, which is a function of three group elements $g_{i}, g_{j}, g_{k} \in G_{b}$. If $n_{2}\left(g_{i}, g_{j}, g_{k}\right)=0$, the Majorana fermions $\gamma_{i j k, A}^{\sigma}$ and $\gamma_{i j k, B}^{\sigma}$ on the two sides of the triangle 


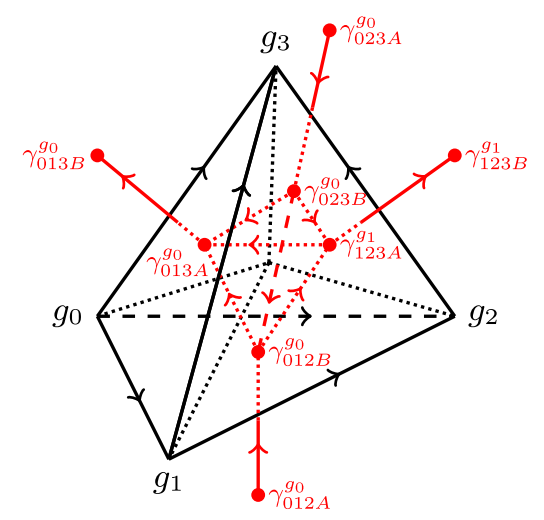

(a)

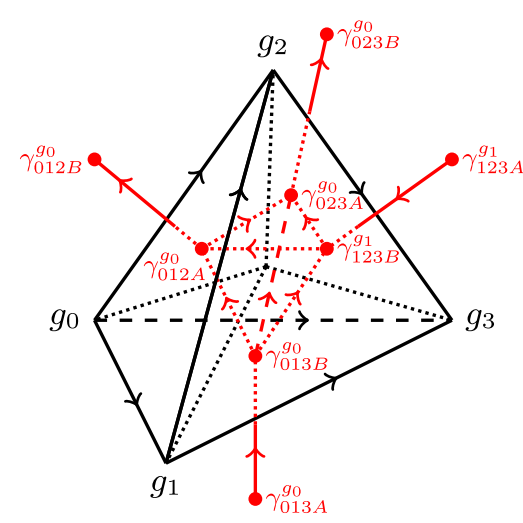

(b)

FIG. 9. Local Kasteleyn orientations of the resolved dual lattice. For a given triangulation of the 3D spatial spin manifold (shown by black links), we can construct a resolved dual lattice (shown by red links). The Majorana fermion pairings (in the standard tetrahedron) should respect the red link arrows in the figures. (a) Positive oriented tetrahedron (b) Negative oriented tetrahedron.

$\langle i j k\rangle$ are in vacuum pairings: $-i \gamma_{i j k, A}^{\sigma} \gamma_{i j k, B}^{\sigma}=1$ (for all $\left.\sigma \in G_{b}\right)$. On the other hand, if $n_{2}\left(g_{i}, g_{j}, g_{k}\right)=1$, we decorate a Kitaev chain going through the triangle $\langle i j k\rangle$. For all $\left|G_{b}\right|$ species of Majorana fermions, we put only $\gamma_{i j k, A}^{g_{i}}$ and $\gamma_{i j k, B}^{g_{i}}$ to be in the nontrivial pairing. All other $\left|G_{b}\right|-1$ species of Majorana fermions $\gamma_{i j k, A}^{\sigma}$ and $\gamma_{i j k, B}^{\sigma}$ with $\sigma \neq g_{i}$ are still in vacuum pairings. Here is an example of the decoration of Kitaev chain going through triangles $\langle 013\rangle$ and $\langle 023\rangle$ of the tetrahedron $\langle 0123\rangle$ (we omit the operator labels of Majorana fermions which are in vacuum pairings along the Kitaev chain):

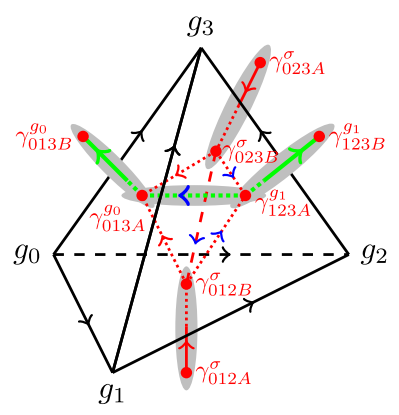

The decorated Kitaev chain is indicated by a green line $\left(\gamma_{013 B}^{g_{0}}-\gamma_{013 A}^{g_{0}}-\gamma_{123 A}^{g_{1}}-\gamma_{123 B}^{g_{1}}\right)$. Along the Kitaev chain, there is a nontrivial pairing between $\gamma_{123 A}^{g_{1}}$ and $\gamma_{013 A}^{g_{0}}$. And the Majorana fermions $\gamma_{013 A / B}^{\sigma}\left(\sigma \neq g_{0}\right)$ and $\gamma_{123 A / B}^{\sigma}\left(\sigma \neq g_{1}\right)$ are all in vacuum pairings. For the triangles without a Kitaev chain going through (triangles $\langle 012\rangle$ and $\langle 023\rangle$ ), the Majorana fermions on their two sides are all in vacuum pairings $\left(\gamma_{012 A / B}^{\sigma}\right.$ and $\gamma_{023 A / B}^{\sigma}$ for all $\left.\sigma \in G_{b}\right)$. In summary, we have the following Majorana fermion pairings:

$$
\gamma_{i j k, A}^{\sigma} \gamma_{i j k, B}^{\sigma} \Longleftrightarrow-i \gamma_{i j k, A}^{\sigma} \gamma_{i j k, B}^{\sigma}=1
$$

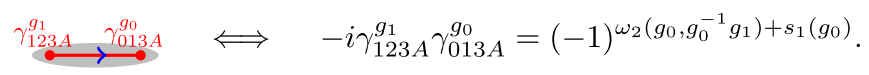

Both the trivial and nontrivial Majorana fermion pairings are indicated by gray ellipses. The blue arrow means that the pairing direction of Majorana fermions may be changed compared to the local Kasteleyn orientation indicated by red arrow. We discuss more about the detailed pairing directions and why they are symmetric later.

b. Consistency condition.-According to our decoration rule, the total number of decorated Kitaev chains going through the four boundary triangles of a given tetrahedron $\langle 0123\rangle$ is

$$
\begin{aligned}
\left(d n_{2}\right)\left(g_{0}, g_{1}, g_{2}, g_{3}\right)= & n_{2}\left(g_{1}, g_{2}, g_{3}\right)+n_{2}\left(g_{0}, g_{2}, g_{3}\right) \\
& +n_{2}\left(g_{0}, g_{1}, g_{3}\right)+n_{2}\left(g_{0}, g_{1}, g_{2}\right) .
\end{aligned}
$$

Since we are constructing a gapped state without intrinsic topological order, there should be no dangling free Majorana fermions inside any tetrahedron. So the number of total Kitaev chains going through the boundary of a tetrahedron should be even. We therefore have the $(\bmod 2)$ equation

$$
d n_{2}=0,
$$

as the consistency condition for Kitaev chain decorations.

c. Symmetric pairing directions. - Now let us turn back to the details of symmetric Majorana fermion pairings inside each tetrahedron of the 3D triangulation lattice. Our strategy of constructing $G_{b}$-symmetric pairings is the same 
as in the 2D case: We first consider the standard tetrahedron with $g_{0}=e$ and then apply a $U\left(g_{0}\right)$ action to obtain all other nonstandard tetrahedra with generic group element labels. In this way, the Majorana fermion pairings are automatically symmetric because of the symmetry transformation rule of the pairing projection operators Eq. (99). For the standard tetrahedron, the Majorana fermions are paired according to the local Kasteleyn orientations indicated by red arrows. And the pairings in the nonstandard tetrahedron is obtained by a $U\left(g_{0}\right)$ action as follows:

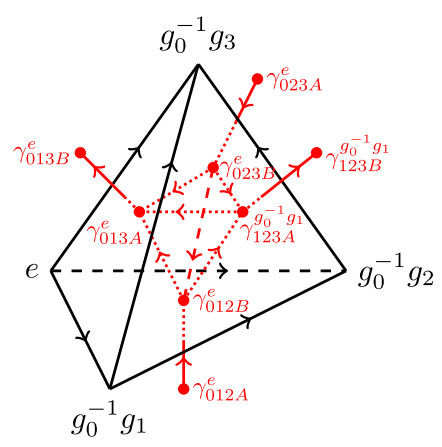

Note that the Majorana fermions $\gamma_{i j k, A}^{\sigma}$ and $\gamma_{i j k, B}^{\sigma}\left(\sigma \neq g_{i}\right)$ on the two sides of triangle $\langle i j k\rangle$ are always in vacuum pairings $\left(-i \gamma_{i j k, A}^{\sigma} \gamma_{i j k, B}^{\sigma}=1\right)$, independent of the $n_{2}$ configurations. So their pairing directions always follow the red arrow local Kasteleyn orientations in both figures of the above equation. For the two Majorana fermions $\gamma_{i j k, A}^{g_{i}}$ and $\gamma_{i j k, B}^{g_{i}}$ of the triangle $\langle i j k\rangle$, there are two possibilities. If $n_{2}\left(g_{i}, g_{j}, g_{k}\right)=0$ (there is no Kitaev chain going through this triangle), these two Majorana fermions are also in vacuum pairing, with the direction indicated by the red arrow and projection operator $P_{i j k A, i j k B}^{g_{i}, g_{i}}=U\left(g_{0}\right) P_{i j k A, i j k B}^{g_{0}^{-1} g_{i}, g_{0}^{-1} g_{i}} U\left(g_{0}\right)^{-1}=\frac{1}{2}\left(1-i \gamma_{i j k, A}^{g_{i}} \gamma_{i j k, B}^{g_{i}}\right)$.

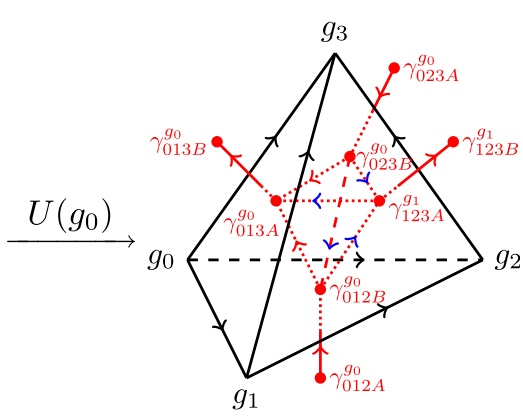

On the other hand, if $n_{2}\left(g_{i}, g_{j}, g_{k}\right)=1$ (there is a Kitaev chain going through this triangle), we pair the Majorana fermion inside the triangle with another one belonging to another triangle with also $n_{2}=1$ [for example, $\gamma_{123 A}^{g_{1}}$ and $\gamma_{013 A}^{g_{0}}$ are paired in Eq. (173)]. Note that there are always an even number of Majorana fermions in nontrivial pairing among the four Majorana fermions $\left(\gamma_{123 A}^{g_{1}}, \gamma_{023 B}^{g_{0}}, \gamma_{013 A}^{g_{0}}\right.$, and $\left.\gamma_{012 B}^{g_{0}}\right)$ inside the tetrahedron $\langle 0123\rangle$, for we have $\left(d n_{2}\right)\left(g_{0}, g_{1}, g_{2}, g_{3}\right)=0(\bmod 2)$ from Eq. (177). There are in total $(4 \times 3) / 2=6$ possible nontrivial pairings inside the tetrahedron $\langle 0123\rangle$ (i.e., the six links of the small red tetrahedron inside the big tetrahedron $\langle 0123\rangle$ ). The Majorana pairing projection operators of them are as follows:

$$
\begin{gathered}
P_{012 B, 013 A}^{g_{0}, g_{0}}=U\left(g_{0}\right) P_{012 B, 013 A}^{e, e} U\left(g_{0}\right)^{\dagger}=\frac{1}{2}\left(1-i \gamma_{012 B}^{g_{0}} \gamma_{013 A}^{g_{0}}\right), \\
P_{023 B, 013 A}^{g_{0}, g_{0}}=U\left(g_{0}\right) P_{023 B, 013 A}^{e, e} U\left(g_{0}\right)^{\dagger}=\frac{1}{2}\left(1-i \gamma_{023 B}^{g_{0}} \gamma_{013 A}^{g_{0}}\right), \\
P_{023 B, 012 B}^{g_{0}, g_{0}}=U\left(g_{0}\right) P_{023 B, 012 B}^{e, e} U\left(g_{0}\right)^{\dagger}=\frac{1}{2}\left[1-(-1)^{s_{1}\left(g_{0}\right)} i \gamma_{023 B}^{g_{0}} \gamma_{012 B}^{g_{0}}\right], \\
P_{023 B, 123 A}^{g_{0}, g_{1}}=U\left(g_{0}\right) P_{023 B, 123 A}^{e, g_{0}^{-1} g_{1}} U\left(g_{0}\right)^{\dagger}=\frac{1}{2}\left[1-(-1)^{\omega_{2}\left(g_{0}, g_{0}^{-1} g_{1}\right)} i \gamma_{023 B}^{g_{0}} \gamma_{123 A}^{g_{1}}\right], \\
P_{012 B, 123 A}^{g_{0}, g_{1}}=U\left(g_{0}\right) P_{012 B, 123 A}^{e, g_{0}^{-1} g_{1}} U\left(g_{0}\right)^{\dagger}=\frac{1}{2}\left[1-(-1)^{\omega_{2}\left(g_{0}, g_{0}^{-1} g_{1}\right)} i \gamma_{012 B}^{g_{0}} \gamma_{123 A}^{g_{1}}\right], \\
P_{123 A, 013 A}^{g_{1}, g_{0}}=U\left(g_{0}\right) P_{123 A, 013 A}^{g_{0}^{-1} g_{1}, e} U\left(g_{0}\right)^{\dagger}=\frac{1}{2}\left[1-(-1)^{\omega_{2}\left(g_{0}, g_{0}^{-1} g_{1}\right)+s_{1}\left(g_{0}\right)} i \gamma_{123 A}^{g_{1}} \gamma_{013 A}^{g_{0}}\right] .
\end{gathered}
$$




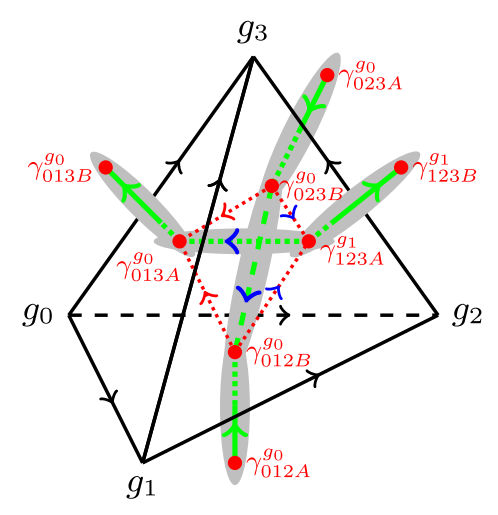

(a)

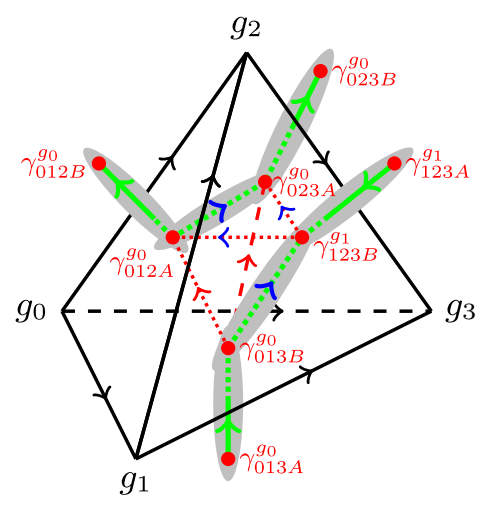

(b)

FIG. 10. Resolvation of four strings of Kitaev chains meeting at one tetrahedron. If four strings (green lines) meet at one tetrahedron, we should pair the Majorana fermions $\gamma_{123 A / B}^{g_{1}}$ to $\gamma_{013 A / B}^{g_{0}}$ and $\gamma_{023 B / A}^{g_{0}}$ to $\gamma_{012 B / A}^{g_{0}}$ (gray ellipses) for a positive or negative oriented tetrahedron. We omit the labels of Majorana fermions $\left(\gamma_{i j k, A / B}^{\sigma}\right.$ with $\left.\sigma \neq g_{i}\right)$ that are in vacuum pairings in the figure. (a) Positive oriented tetrahedron (b) Negative oriented tetrahedron.

Among the six possible nontrivial pairings, only the last four may change their directions in the nonstandard triangle. They are indicated by blue arrows in the righthand-side figure in Eq. (178). This result can be understood from the following facts from the symmetry transformation on projection operators Eq. (98): The $(-1)^{\omega_{2}}$ term appears in the projection operators when the pairing is between Majorana fermions with different group element labels [see Eqs. (183)-(185)], and the $(-1)^{s_{1}}$ term appears when the pairing is between the same $A / B$-type Majorana fermions [see Eqs. (182) and (185)]. The first two pairings [Eqs. (180) and (181)] belong to neither of the above two cases. So their pairing direction is the same as the red arrow Kasteleyn orientations even after $U\left(g_{0}\right)$ action.

There is another subtlety when $n_{2}=1$ for all four triangles of a tetrahedron. There are four strings of Kitaev chains meeting at the tetrahedron $\langle 0123\rangle$. In this case, we should resolve the crossing point of the four strings. We use the convention that the Majorana fermions $\gamma_{123 A}^{g_{1}}$ and $\gamma_{013 A}^{g_{0}}$ are paired and $\gamma_{023 B}^{g_{0}}$ and $\gamma_{012 B}^{g_{0}}$ are paired [see Fig. 10(a)]. Of course, all other Majorana fermions $\gamma_{i j k, A / B}^{\sigma}$ with $\sigma \neq g_{i}$ are still in vacuum pairings. This resolvation convention is the same as Ref. [53].

d. Majorana fermion parity.-Since the symmetry action may change the pairing directions inside a tetrahedron, the Majorana fermion parity of this tetrahedron may also be changed. We can calculate the fermion parity difference between the standard and nonstandard tetrahedra by counting the number of pairing arrows that are reversed by $U\left(g_{0}\right)$ action. It, of course, depends on the $n_{2}$ configurations. We can use, for example, $n_{2}\left(g_{0}, g_{1}, g_{2}\right) n_{2}\left(g_{1}, g_{2}, g_{3}\right)=0,1$ to indicate whether $\gamma_{012 B}^{g_{1}}$ and $\gamma_{123 A}^{g_{1}}$ are paired or not. So the Majorana fermion parity change inside the triangle is, in general, given by

$$
\begin{aligned}
& \Delta P_{f}^{\gamma}(0123)=(-1)^{\omega_{2}\left(g_{0}, g_{0}^{-1} g_{1}\right) n_{2}\left(g_{1}, g_{2}, g_{3}\right)+s_{1}\left(g_{0}\right)\left[n_{2}\left(g_{0}, g_{2}, g_{3}\right) n_{2}\left(g_{0}, g_{1}, g_{2}\right)+n_{2}\left(g_{0}, g_{1}, g_{3}\right) n_{2}\left(g_{1}, g_{2}, g_{3}\right)\right]} \\
& =(-1)^{\left[\omega_{2} \smile n_{2}+s_{1} \smile\left(n_{2} \smile{ }_{1} n_{2}\right)\right]\left(g_{0}, g_{0}^{-1} g_{1}, g_{1}^{-1} g_{2}, g_{2}^{-1} g_{3}\right)},
\end{aligned}
$$

where we use the higher cup product definition $\left(n_{2} \smile 1 n_{2}\right)(0123)=n_{2}(023) n_{2}(012)+n_{2}(013) n_{2}(123)$. The above equation is a summary of the phase factors appearing in Eqs. (180)-(185). The first term $(-1)^{\omega_{2}}$ appears iff $n_{2}\left(g_{1}, g_{2}, g_{3}\right)=1$. And the second term $(-1)^{s_{1}\left(g_{0}\right)}$ in the first line of Eq. (186) appears when $n_{2}\left(g_{0}, g_{2}, g_{3}\right) n_{2}\left(g_{0}, g_{1}, g_{2}\right)=1 \quad$ or $\quad n_{2}\left(g_{0}, g_{1}, g_{3}\right) n_{2}\left(g_{1}\right.$, $\left.g_{2}, g_{3}\right)=1$. Note that, different from the $2 \mathrm{D}$ case, we cannot find a single Majorana fermion involving all the Majorana fermion parity configurations.
The above discussions of Majorana fermion pairings also apply to the negative oriented tetrahedra. The red arrow local Kasteleyn orientations inside a tetrahedron are given in Fig. 9(b). The symmetric Majorana fermion pairing in a nonstandard tetrahedron is also obtained by a $U\left(g_{0}\right)$ action from the standard tetrahedron. We also have Majorana pairing projection operators similar to Eqs. (180)-(185) for negative oriented tetrahedron. For $n_{2}$ configurations with four Kitaev chains meeting at one negative oriented tetrahedron, we use the resolvation convention shown in 
Fig. 10(b). And the expression of Majorana fermion parity changes Eq. (186) is valid for both positive and negative oriented tetrahedra.

To sum up, although there are $\left|G_{b}\right|$ species of Majorana fermions, we decorate exactly one Kitaev chain to each intersection line of symmetry domain walls specified by $n_{2}$ configurations. The decoration is compatible with symmetry actions. The Majorana fermion parity of a tetrahedron is changed according to Eq. (186) compared to the local Kasteleyn oriented pairings.

\section{Complex fermion decoration}

The rules of complex fermion decoration are much simpler than that of the Majorana fermions. The decoration is specified by a $\mathbb{Z}_{2}$-valued 3-cochain $n_{3} \in C^{1}\left(G_{b}, \mathbb{Z}_{2}\right)$. If $n_{3}\left(g_{i}, g_{j}, g_{k}, g_{l}\right)=0$, all the modes of complex fermions $c_{i j k l}^{\sigma}\left(\sigma \in G_{b}\right)$ at the center of tetrahedron $\langle i j k l\rangle$ $(i<j<k<l)$ are unoccupied. On the other hand, if $n_{3}\left(g_{i}, g_{j}, g_{k}, g_{l}\right)=1$, exactly one complex fermion mode $c_{i j k l}^{g_{i}}$ is decorated at the center of tetrahedron $\langle i j k l\rangle$ [see
Fig. 8(a)]. All other complex fermions $c_{i j k l}^{\sigma}\left(\sigma \neq g_{i}\right)$ are still in vacuum states.

It is simple to check that the complex fermion decoration is $G_{b}$ symmetric. Under a $U(g)$ action, the bosonic vertex label $\left\{g_{i}\right\}$ becomes $\left\{g g_{i}\right\}$. And we should decorate $c_{i j k l}^{g g_{i}}$ to the tetrahedron $\langle i j k l\rangle$ if $n_{3}\left(g g_{i}, g g_{j}, g g_{k}, g g_{l}\right)=$ $n_{3}\left(g_{i}, g_{j}, g_{k}, g_{l}\right)=1$. This result is exactly the complex fermion $c_{i j k l}^{g_{i}}$ by a $U(g)$ action.

\section{C. $F$ moves}

The 3D Pachner move for different triangulations of 3D spatial manifold induces FSLU transformation of the FSPT wave functions on the lattices. Since the FSPT state is invertible, we need to consider only one of the many Pachner moves. Other Pachner moves can be derived from this one using the invertibility and unitarity of the move. We can first define the standard $F$ move with $g_{0}=e$, and then other nonstandard ones can be obtained by simply a $U\left(g_{0}\right)$ action. The standard $F$ move is given by

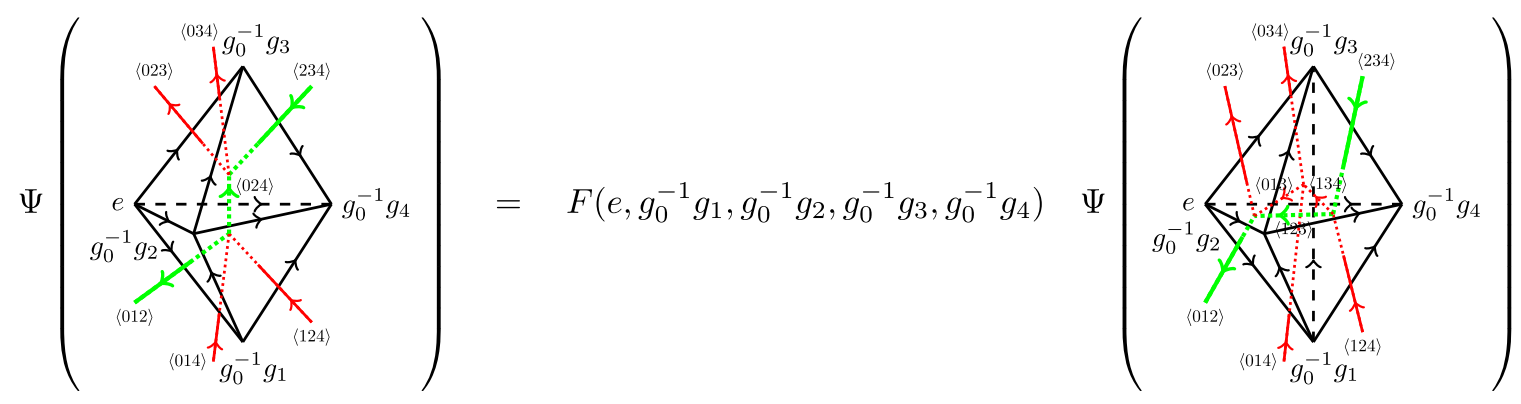

on the (black) triangulation lattice and

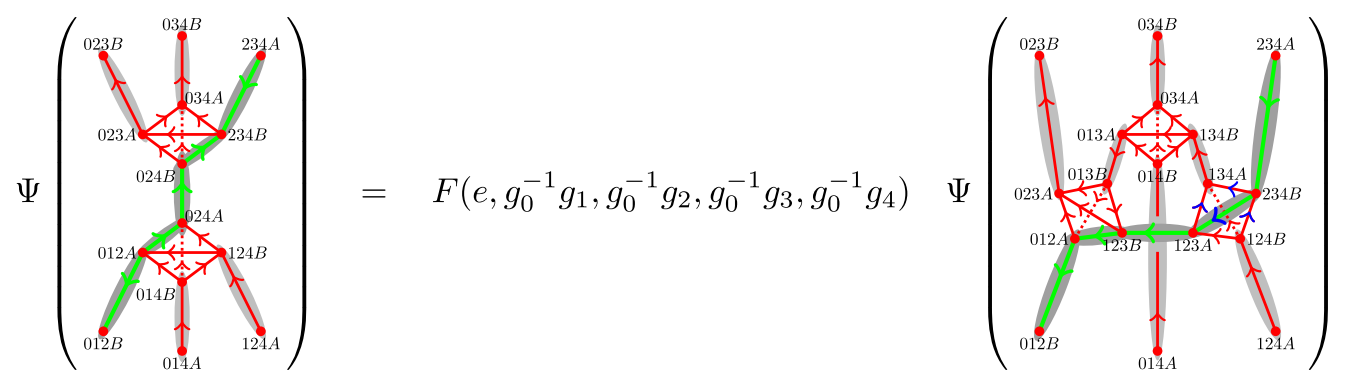

on the (red) resolved dual lattice. This Pachner move involves five vertices from $\langle 0\rangle$ to $\langle 4\rangle$, with group element labels $e, g_{0}^{-1} g_{1}, g_{0}^{-1} g_{2}, g_{0}^{-1} g_{3}$, and $g_{0}^{-1} g_{4}$ in $G_{b}$. There are three tetrahedra $(\langle 0123\rangle,\langle 0134\rangle$, and $\langle 1234\rangle)$ in the righthand-side figure and two tetrahedra $(\langle 0124\rangle$ and $\langle 0234\rangle)$ in the left-hand-side figure in Eq. (187). The Pachner move removes the link $\langle 13\rangle$ on the right. Note that we omit the $p+i p$ layer in the above figures. And for each tetrahedron, the meeting point of the four red or green strings in Eq. (188) should be resolved to a small tetrahedron with local Kasteleyn orientations in Eq. (188) [using the convention Fig. 9(b)]. The blue arrows of the red links inside the only nonstandard tetrahedron $\langle 1234\rangle$ [see the righthand-side figure in Eq. (188)] mean that their directions 
may be changed under $U\left(g_{0}^{-1} g_{1}\right)$ action compared to the red arrow local Kasteleyn orientations [see Eq. (178)]. The green line represents the decorated Kitaev chain specified by the $n_{2}$ data. We also omit the blue ball symbols for the decorated complex fermions at the center of the tetrahedra in the figures.

The explicit expression of the standard FSLU $F$ operator for the above Pachner move is

$$
F(e, \overline{0} 1, \overline{0} 2, \overline{0} 3, \overline{0} 4)=\nu_{4}(\overline{0} 1, \overline{1} 2, \overline{2} 3, \overline{3} 4)\left(c_{0124}^{e^{\dagger}}\right)^{n_{3}(0124)}\left(c_{0234}^{e \dagger}\right)^{n_{3}(0234)}\left(c_{0123}^{e}\right)^{n_{3}(0123)}\left(c_{0134}^{e}\right)^{n_{3}(0134)}\left(c_{1234}^{g_{0}^{-1} g_{1}}\right)^{n_{3}(1234)} X_{01234}\left[n_{2}\right]
$$

We use the abbreviation $\bar{i} j$ for $g_{i}^{-1} g_{j}$ in the arguments of $F$ and $\nu_{4}$. Different from $\nu_{4}$, the 3-cochain $n_{3}$ is symmetric under $G_{b}$ action. So there is no difference between homogeneous and inhomogeneous $n_{3}$. We use $n_{3}(i j k l)$ to represent $n_{3}\left(g_{i}, g_{j}, g_{k}, g_{l}\right)=n_{3}\left(g_{i}^{-1} g_{j}, g_{j}^{-1} g_{k}, g_{k}^{-1} g_{l}\right)$ for short. In the following, we explain the terms in the $F$ operator in Eq. (189) one by one. There is some subtlety about the Majorana fermion parity changes for the $X$ operator. We give the explicit expression for $X$ in the next subsection.

The $U(1)$ phase factor $\nu_{4}(\overline{0} 1, \overline{1} 2, \overline{2} 3, \overline{3} 4)=\nu_{4}\left(g_{0}^{-1} g_{1}\right.$, $\left.g_{1}^{-1} g_{2}, g_{2}^{-1} g_{3}, g_{3}^{-1} g_{4}\right)$ in the front of the $F$ symbol is an inhomogeneous 4-cochain depending on four group elements of $G_{b}$. By definition, the inhomogeneous cochain is related to the homogeneous one by

$$
\begin{aligned}
& \nu_{4}\left(g_{0}^{-1} g_{1}, g_{1}^{-1} g_{2}, g_{2}^{-1} g_{3}, g_{3}^{-1} g_{4}\right) \\
& \quad=\nu_{4}\left(e, g_{0}^{-1} g_{1}, g_{0}^{-1} g_{2}, g_{0}^{-1} g_{3}, g_{0}^{-1} g_{4}\right),
\end{aligned}
$$

with the first argument of the homogeneous $\nu_{4}$ the identity element $e \in G_{b}$. As discussed below, we can relate $\nu_{4}\left(e, g_{0}^{-1} g_{1}, g_{0}^{-1} g_{2}, g_{0}^{-1} g_{3}, g_{0}^{-1} g_{4}\right)$ and $\nu_{4}\left(g_{0}, g_{1}, g_{2}, g_{3}, g_{4}\right)$ from the symmetry conditions of $F$. Different from the special case $G_{f}=\mathbb{Z}_{f} \times G_{b}$, they do not equal each other, in general.

The complex fermion term of the form $c^{\dagger} c^{\dagger} c c c$ annihilates three complex fermions at the three tetrahedra of the right-hand-side figure and creates two in the left-hand-side figure in Eq. (187). Following Sec. VI B 2, the tetrahedron $\langle i j k l\rangle$ is decorated by complex fermion $c_{i j k l}^{g_{i}}$. So, in the standard $F$ move, only the last fermion $c_{1234}^{g_{0}^{-1} g_{1}}$ has group element label $g_{0}^{-1} g_{1}$, and all other four fermions have group element label $e$. We note that, different from the special case $G_{f}=\mathbb{Z}_{2}^{f} \times G_{b}$ where we have $d n_{3}=n_{2} \smile n_{2}$ [53], the complex fermion number $n_{3}$ has a more complicated relation with the $n_{2}$ data, in general.

\section{Majorana fermion parity and $X$ operator}

In this subsection, we give an explicit expression for the $X$ operator in the standard $F$ move Eq. (189), which is related to the Kitaev chain decorations.
As a consequence of the local Kasteleyn orientation of the red lattice, the Majorana fermion parities for the two Kitaev chain decorated states on the two sides of Eq. (187) may be different. It is shown in Ref. [53] that, if the Majorana fermions are paired according to the local Kasteleyn orientations, the Majorana fermion parity difference under the $F$ move is

$$
\left.\Delta P_{f}^{\gamma}(F)\right|_{s_{1}=\omega_{2}=0}=(-1)^{\left(n_{2} \smile n_{2}\right)(01234)} .
$$

So, among all the Kitaev chain decoration configurations of the $F$ move, only the ones with $n_{2}(012)=n_{2}(234)=1$ change the Majorana fermion parity [see the green lines in Eq. (187) for an example]. However, for a generic symmetry group with nonzero $s_{1}$ and $\omega_{2}$, the Majorana fermions are paired according to the rules designed in Sec. VI B 1. As shown by blue arrows in Eq. (178), the pairing directions inside the nonstandard tetrahedron may be changed by the symmetry action compared to the local Kasteleyn orientations.

In the standard $F$ move Eq. (187), there are in total five relevant tetrahedra. Four of these tetrahedra are standard, with the group element label of the first vertex $e \in G_{b}$. However, the tetrahedron $\langle 1234\rangle$ on the right-hand side in Eq. (187) is nonstandard and has first vertex label $g_{0}^{-1} g_{1}$. Therefore, only inside the tetrahedron $\langle 1234\rangle$ of the standard $F$ move may the pairing directions of the Majorana fermions be changed. The pairings are given by the projection operators Eqs. (180)-(185), with the replacement $\left(g_{0}, g_{1}, g_{2}, g_{3}\right) \rightarrow\left(g_{0}^{-1} g_{1}, g_{0}^{-1} g_{2}, g_{0}^{-1} g_{3}, g_{0}^{-1} g_{4}\right)$. Furthermore, as discussed also in Sec. VI B 1, the Majorana fermion parity change for a nonstandard tetrahedron $\langle 0123\rangle$ compared to the local Kasteleyn orientations is given by Eq. (186). For the tetrahedron $\langle 1234\rangle$ of the standard $F$ move, the Majorana fermion parity change compared to the local Kasteleyn orientations is then

$\Delta P_{f}^{\gamma}(1234)=(-1)^{\left[\omega_{2} \smile n_{2}+s_{1} \smile\left(n_{2} \smile_{1} n_{2}\right)\right]\left(g_{0}^{-1} g_{1}, g_{1}^{-1} g_{2}, g_{2}^{-1} g_{3}, g_{3}^{-1} g_{4}\right)}$.

This result is obtained from Eq. (186) by the simple replacement $\left(g_{0}, g_{1}, g_{2}, g_{3}\right) \rightarrow\left(g_{0}^{-1} g_{1}, g_{0}^{-1} g_{2}, g_{0}^{-1} g_{3}, g_{0}^{-1} g_{4}\right)$. Combining it with Eq. (191), the total Majorana fermion parity change under the standard $F$ move Eq. (187) is 


$$
\Delta P_{f}^{\gamma}(F)=(-1)^{\left[n_{2} \smile n_{2}+\omega_{2} \smile n_{2}+s_{1} \smile\left(n_{2} \smile n_{2}\right)\right](01234)} .
$$

We note that there is no difference between the homogeneous and inhomogeneous notations for $\mathbb{Z}_{2}$-valued cocycles $s_{1}$ and $\omega_{2}$ and cochain $n_{2}$, for they are symmetric under $G_{b}$ action. So we can just use (01234) to represent $\left(g_{0}, g_{1}, g_{2}, g_{3}, g_{4}\right)$ or $\left(g_{0}^{-1} g_{1}, g_{1}^{-1} g_{2}, g_{2}^{-1} g_{3}, g_{3}^{-1} g_{4}\right)$ in the above equation. This situation is very different from the $U(1)$ phase factor $\nu_{4}$.

It is convenient to split the exponent of $(-1)$ in Eq. (193) into two parts:

$$
\begin{aligned}
\alpha_{4}(01234):= & \left(n_{2} \smile n_{2}+\omega_{2} \smile n_{2}\right)(01234) \\
& +s_{1}(01) n_{2}(124) n_{2}(234) \quad(\bmod 2),
\end{aligned}
$$

$\beta_{4}(01234):=s_{1}(01) n_{2}(134) n_{2}(123) \quad(\bmod 2)$.

Their summation gives the total Majorana fermion parity change number

$\alpha_{4}+\beta_{4}=n_{2} \smile n_{2}+\omega_{2} \smile n_{2}+s_{1} \smile\left(n_{2} \smile n_{1} n_{2}\right) \quad(\bmod 2)$.

The philosophy of this splitting is as follows. The first part $\alpha_{4}$ has the property that $\alpha_{4}(01234)=1$ implies $n_{2}(234)=1$, because all of the terms in $\alpha_{4}(01234)$ contain the factor $n_{2}(234)$. However, the second part $\beta_{4}$ does not contain the factor $n_{2}(234)$. For a given Kitaev chain configuration $n_{2}$, if $\alpha_{4}(01234)=1$ and $\beta_{4}(01234)=0$, we can conclude that the Majorana fermion parity of the Kitaev chain going through the link $\langle 234\rangle$ is changed. We can add a Majorana fermion operator $\gamma_{234 B}^{g_{0}^{-1} g_{2}}$ to the $X$ operator of Eq. (189) to represent the correct fermion parity change. On the other hand, if $\alpha_{4}(01234)=0$ and $\beta_{4}(01234)=1$, the Majorana fermion parity of the Kitaev chain going through the link $\langle 123\rangle$ is changed. We can add another Majorana fermion operator $\gamma_{123 A}^{g_{0}^{-1} g_{1}}$ to the $X$ operator (we can also use $\gamma_{134 A}^{g_{0}^{-1} g_{1}}$ as another convention). In this case, adding $\gamma_{234 B}^{g_{0}^{-1} g_{2}}$ does not make sense, because the Kitaev chain may not even go through this Majorana fermion.

With the above understanding, the explicit form of $X_{01234}\left[n_{2}\right]$ in the standard $F$ move Eq. (189) can be expressed by Majorana fermion pairing projection operators as

$$
X_{01234}\left[n_{2}\right]=P_{01234}\left[n_{2}\right] \cdot\left(\gamma_{234 B}^{g_{0}^{-1} g_{2}}\right)^{\alpha_{4}(01234)}\left(\gamma_{123 A}^{g_{0}^{-1} g_{1}}\right)^{\beta_{4}(01234)},
$$

$$
\begin{aligned}
P_{01234}\left[n_{1}\right]= & \left(\prod_{\text {loopi }} 2^{\left(L_{i}-1\right) / 2}\right)\left(\prod_{\text {Majorana pairs }\langle a, b\rangle \text { in } \mathcal{T}} P_{a, b}^{g_{a}, g_{b}}\right) \\
& \times\left(\prod_{\text {triangle }\langle i j k\rangle \notin \mathcal{T}} \prod_{\sigma \in G_{b}} P_{i j k A, i j k B}^{\sigma, \sigma}\right) .
\end{aligned}
$$

The two Majorana fermion operators $\gamma_{234 B}^{g^{-1} g_{2}}$ and $\gamma_{123 A}^{g_{0}^{-1} g_{1}}$ are inserted when $\alpha_{4}(01234)=1$ and $\beta_{4}(01234)=1$, respectively. The first part $P_{01234}\left[n_{1}\right]$ in the $X$ operator Eq. (197) is the Majorana fermion pairing projection operator, which enforces the symmetric pairing rules for the left-hand-side Majorana state in Eq. (187). Similar to the 2D case, the general expression of $P_{01234}\left[n_{1}\right]$ [Eq. (198)] has three terms. The first term is a normalization factor, where $2 L_{i}$ is the length of the $i$ th loop in the transition graph of Majorana pairing dimer configurations on the left triangulation lattice $\mathcal{T}$ and right lattice $\mathcal{T}^{\prime}$. The second term projects the righthand-side state to the left-hand-side state using the pairing projection operators [Eq. (98)]. And the third term is the vacuum projection operators for the Majorana fermions that do not appear explicitly in the left figure. For example, the explicit $X$ operator for the $n_{2}$ configurations shown in Eq. (187) [only $n_{2}(012)=n_{2}(024)=n_{2}(123)=$ $\left.n_{2}(234)=1\right]$ is

$$
\begin{aligned}
X_{01234}\left[\tilde{n}_{2}\right]= & 2\left(P_{024 B, 234 B}^{e, g_{0}^{-1} g_{2}} P_{012 A, 024 A}^{e, e} \prod_{\sigma \neq e} P_{024 A, 024 B}^{\sigma, \sigma}\right) \\
& \times\left(\prod_{\sigma \in G_{b}} P_{013 A, 013 B}^{\sigma, \sigma} P_{123 A, 123 B}^{\sigma, \sigma} P_{134 A, 134 B}^{\sigma, \sigma}\right) \\
& \times\left(\gamma_{234 B}^{g_{0}^{-1} g_{2}}\right)^{\alpha_{4}(01234)},
\end{aligned}
$$

where we do not need $\gamma_{123 A}^{g_{0}^{-1} g_{1}}$, because the configuration $n_{2}(134)=0$ implies $\beta_{4}(01234)=s_{1}(01) n_{2}(134) n_{2}$ $(123)=0$.

The $F$ symbol in Eq. (189) should be a FSLU operator. It should be both fermion parity even and symmetric under $G_{b}$ action. We can use these constraints to obtain several consistency equations for the cochains $n_{2}, n_{3}$, and $\nu_{4}$.

\section{Fermion parity conservation}

As discussed in the previous subsection, the Majorana fermion parity change for the standard $F$ move is given by Eq. (191), if the pairings are according to the local Kasteleyn orientations. From the symmetry action on the only nonstandard tetrahedron $\langle 1234\rangle$ of the standard $F$ move, there is an additional Majorana fermion parity change [Eq. (192)]. By combining them, we obtain the total Majorana fermion parity change [Eq. (193)] for the standard $F$ move.

On the other hand, the complex fermion parity change under the standard $F$ move can be simply calculated by counting the complex fermions decorated at the five tetrahedra on the two sides: 


$$
\begin{aligned}
\Delta P_{f}^{c}(F) & =(-1)^{n_{3}(1234)+n_{3}(0234)+n_{3}(0134)+n_{3}(0124)+n_{3}(0123)} \\
& =(-1)^{d n_{3}(01234)}
\end{aligned}
$$

As a FSLU transformation, the standard $F$ move should preserve the total fermion parity. So we have the constraint $\Delta P_{f}(F)=\Delta P_{f}^{\gamma}(F) \cdot \Delta P_{f}^{c}(F)=1$. Using the explicit expressions Eqs. (193) and (200), we have the following $(\bmod 2)$ equation:

$$
d n_{3}=n_{2} \smile n_{2}+\omega_{2} \smile n_{2}+s_{1} \smile\left(n_{2} \smile{ }_{1} n_{2}\right) .
$$

The decorations of Majorana fermions and complex fermions are not independent and should satisfy the above constraint.

We note that, if we consider the special case of unitary symmetry group $G_{f}=\mathbb{Z}_{2}^{f} \times G_{b}$ (i.e., $\omega_{2}=0$ and $s_{1}=0$ ), the above equation is reduced to the previous known result $d n_{3}=n_{2} \smile n_{2}[53,62,63]$.

\section{Symmetry condition}

In the previous constructions, we considered only the standard $F$ move Eq. (187) with the first vertex label $e \in G_{b}$. The nonstandard $F$ move is defined to be obtained from the standard one by a $U\left(g_{0}\right)$ symmetry action. In such a way, the $F$ moves are symmetric under $G_{b}$ actions. In this subsection, we derive the symmetry transformation rules for the $F$ move and the $U(1)$ phase factor $\nu_{4}$ in front of the $F$ move.

According to Eq. (66), we have the following commuting diagram for the standard and nonstandard $F$ moves (we omit the decorated fermion layers in the figures):

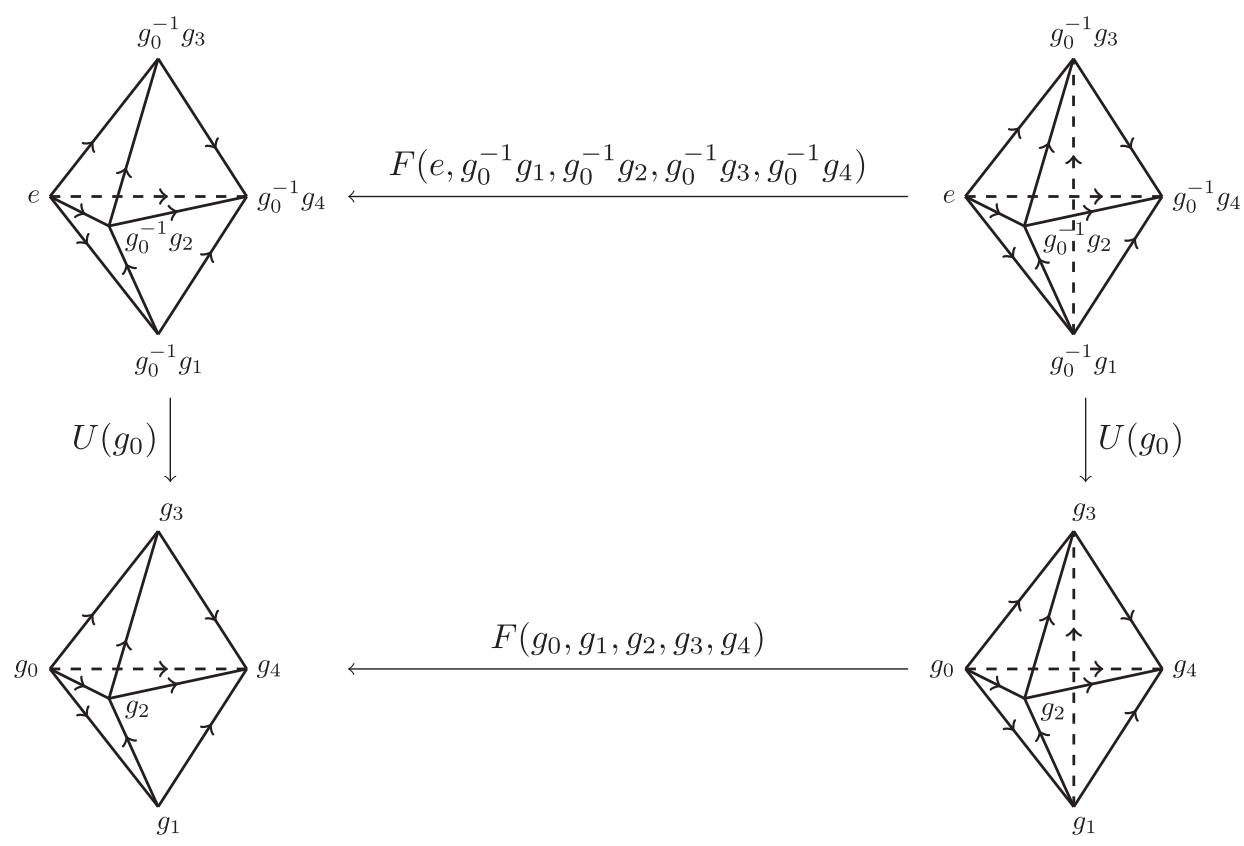

So the nonstandard $F$ operator is defined as

$$
F\left(g_{0}, g_{1}, g_{2}, g_{3}, g_{4}\right)={ }^{g_{0}} F\left(e, g_{0}^{-1} g_{1}, g_{0}^{-1} g_{2}, g_{0}^{-1} g_{3}, g_{0}^{-1} g_{4}\right):=U\left(g_{0}\right) F\left(e, g_{0}^{-1} g_{1}, g_{0}^{-1} g_{2}, g_{0}^{-1} g_{3}, g_{0}^{-1} g_{4}\right) U\left(g_{0}\right)^{\dagger} \text {. }
$$

The nonstandard $F$ moves constructed in this way are automatically symmetric, because one can show the transformation rule for the nonstandard $F$ moves

$$
F\left(g g_{0}, g g_{1}, g g_{2}, g g_{3}, g g_{4}\right)=U(g) F\left(g_{0}, g_{1}, g_{2}, g_{3}, g_{4}\right) U(g)^{\dagger},
$$

using Eq. (63) and the fact that $F$ operator is fermion parity even.

Using a $U\left(g_{0}\right)$ action on the standard $F$ operator Eq. (189), we can obtain the nonstandard $F$ symbol expression as

$$
\begin{aligned}
& F\left(g_{0}, g_{1}, g_{2}, g_{3}, g_{4}\right) \\
& \quad=\nu_{4}\left(g_{0}, g_{1}, g_{2}, g_{3}, g_{4}\right)\left(c_{0124}^{g_{0} \dagger}\right)^{n_{3}(0124)}\left(c_{0234}^{g_{0} \dagger}\right)^{n_{3}(0234)}\left(c_{0123}^{g_{0}}\right)^{n_{3}(0123)}\left(c_{0134}^{g_{0}}\right)^{n_{3}(0134)}\left(c_{1234}^{g_{1}}\right)^{n_{3}(1234)} X_{01234}\left[n_{2}\right] Y_{01234}\left[n_{1}\right] .
\end{aligned}
$$


The decorated complex fermions now have group element labels $g_{0}$ (for the first four complex fermions) or $g_{1}$ (for the last complex fermion). And the $X$ operator is

$$
X_{01234}\left[n_{2}\right]=P_{01234}\left[n_{2}\right] \cdot\left(\gamma_{234 B}^{g_{2}}\right)^{\alpha_{4}(01234)}\left(\gamma_{123 A}^{g_{1}}\right)^{\beta_{4}(01234)},
$$

with added Majorana fermion $\gamma_{234 B}^{g_{2}}$ and $\gamma_{123 A}^{g_{1}}$, rather than $\gamma_{234 B}^{g_{0}^{-1} g_{2}}$ and $\gamma_{123 A}^{g_{0}^{-1} g_{1}}$ in Eq. (197). The operator $P_{01234}\left[n_{2}\right]$ projects the Majorana fermions to the pairing state on the left-hand-side figure. It has a similar expression as Eq. (198) and is a product of many Majorana pairing projection operators [Eq. (98)] with appropriate group element labels. The operator $Y_{01234}\left[n_{1}\right]$ in Eq. (205) is an operator to change the $p+i p$ superconductor decoration configurations. We discuss this layer of decoration in Sec. VI F separately.

From the decoration rules of Majorana fermions and complex fermions constructed in previous sections, the data $n_{1}, n_{2}$, and $n_{3}$ are invariant under $G_{b}$ symmetry actions. The homogeneous cochain $\nu_{4}$ in the nonstandard $F$ move Eq. (205) is a combination of the inhomogeneous $\nu_{4}$ in the standard $F$ move Eq. (189) and the \pm 1 signs which appear from the symmetry action. Therefore, we have the following symmetry conditions for the data $n_{1}, n_{2}, n_{3}$, and $\nu_{4}$ :

$$
\begin{gathered}
n_{1}\left(g_{0}, g_{1}\right)=n_{1}\left(e, g_{0}^{-1} g_{1}\right)=n_{1}\left(g_{0}^{-1} g_{1}\right), \\
n_{2}\left(g_{0}, g_{1}, g_{2}\right)=n_{2}\left(e, g_{0}^{-1} g_{1}, g_{0}^{-1} g_{2}\right)=n_{2}\left(g_{0}^{-1} g_{1}, g_{1}^{-1} g_{2}\right), \\
n_{3}\left(g_{0}, g_{1}, g_{2}, g_{3}\right)=n_{3}\left(e, g_{0}^{-1} g_{1}, g_{0}^{-1} g_{2}, g_{0}^{-1} g_{3}\right)=n_{3}\left(g_{0}^{-1} g_{1}, g_{1}^{-1} g_{2}, g_{2}^{-1} g_{3}\right), \\
\nu_{4}\left(g_{0}, g_{1}, g_{2}, g_{3}, g_{4}\right)={ }^{g_{0}} \nu_{4}\left(e, g_{0}^{-1} g_{1}, g_{0}^{-1} g_{2}, g_{0}^{-1} g_{3}, g_{0}^{-1} g_{4}\right)={ }_{0} \nu_{4}\left(g_{0}^{-1} g_{1}, g_{1}^{-1} g_{2}, g_{2}^{-1} g_{3}, g_{3}^{-1} g_{4}\right) \\
=\nu_{4}\left(g_{0}^{-1} g_{1}, g_{1}^{-1} g_{2}, g_{2}^{-1} g_{3}, g_{3}^{-1} g_{4}\right)^{1-2 s_{1}\left(g_{0}\right)} \cdot \mathcal{O}_{5}^{\text {symm }}\left(g_{0}, g_{1}, g_{2}, g_{3}, g_{4}\right) .
\end{gathered}
$$

The last equation can be viewed as the definition of homogeneous $\nu_{4}$ in the nonstandard $F$ move Eq. (205) in terms of the inhomogeneous $\nu_{4}$ in the standard $F$ move Eq. (189). The symmetry sign difference $\mathcal{O}_{5}^{\text {symm }}$ is given by

$$
\begin{aligned}
\mathcal{O}_{5}^{\mathrm{symm}}\left(g_{0}, g_{1}, g_{2}, g_{3}, g_{4}\right) & =(-1)^{\omega_{2}(0, \overline{0} 1) n_{3}(1234)+\left[\omega_{2}(0, \overline{0} 2)+s_{1}(0)\right] \alpha_{4}(01234)+\omega_{2}(0, \overline{0} 1) \beta_{4}(01234)} \\
& =(-1)^{\left(\omega_{2} \smile n_{3}+s_{1} \smile \alpha_{4}\right)(0, \overline{0} 1, \overline{1} 2, \overline{2} 3, \overline{3} 4)+\omega_{2}(0, \overline{0} 2) \alpha_{4}(01234)+\omega_{2}(0, \overline{0} 1) \beta_{4}(01234)} .
\end{aligned}
$$

We note that some of the terms above cannot be expressed as a cup product form. The above equation can be obtained by straightforward calculation using Eq. (203). In the first line of Eq. (211), the first term of the form $(-1)^{\omega_{2} n_{3}}$ comes from the $U\left(g_{0}\right)$ symmetry transformation Eq. (168) of the last complex fermion $c_{1234}^{g_{0}^{-1} g_{1}}$ in the standard $F$ move Eq. (189). The second term of the form $(-1)^{\left(\omega_{2}+s_{1}\right) \alpha_{4}}$ comes from the symmetry transformation Eq. (170) of $\gamma_{234 B}^{g_{0}^{-1} g_{2}}$ in the $X$ operator Eq. (197) of the standard $F$ move Eq. (189). And the last sign of the form $(-1)^{\omega_{2} \beta_{4}}$ comes from the symmetry transformation Eq. (169) of $\gamma_{123 A}^{g_{0}^{-1} g_{1}}$ in the $X$ operator Eq. (197) of the standard $F$ move. The 4cochains $\alpha_{4}$ and $\beta_{4}$ are defined in Eqs. (194) and (195).

If we have $\beta_{4}=0$ (for example, $s_{1}=0$ or $n_{1}=0$ ), we need only to insert the dangling Majorana fermion operator $\gamma_{234 B}^{g_{0}^{-1} g_{2}}$ in the standard $X$ operator. The expressions of the standard $F$ move Eq. (189) and $X$ operator Eq. (197) are similar to the special case of $s_{1}=\omega_{2}=0$ [53]. Using $d n_{3}=\alpha_{4}+\beta_{4}=\alpha_{4}$, the symmetry sign Eq. (211) is reduced to

$$
\begin{aligned}
\left.\mathcal{O}_{5}^{\text {symm }}\left(g_{0}, g_{1}, g_{2}, g_{3}, g_{4}\right)\right|_{\beta_{4}=0} & =(-1)^{\omega_{2}(0, \overline{0} 1) n_{3}(1234)+\left[\omega_{2}(0, \overline{0} 2)+s_{1}(0)\right] d n_{3}(01234)} \\
& =(-1)^{\left(\omega_{2} \smile n_{3}+s_{1} \smile d n_{3}\right)(0, \overline{0} 1, \overline{1} 2, \overline{2} 3, \overline{3} 4)+\omega_{2}(0, \overline{0} 2) d n_{3}(01234)} .
\end{aligned}
$$

The symmetry sign $\mathcal{O}_{5}^{\text {symm }}$ appears later in the twisted cocycle equation for $\nu_{4}$ as part of the obstruction function [see Eq. (215)]. In the special case of $\beta_{4}=0$, the calculation of $\mathcal{O}_{5}$ is much simpler than the generic case.

\section{Superfusion hexagon and twisted cocycle equations}

Just as the 2D $F$ move should satisfy the superpentagon equation of the superfusion category, the 3D $F$ move should satisfy a superhexagon equation of the superfusion 


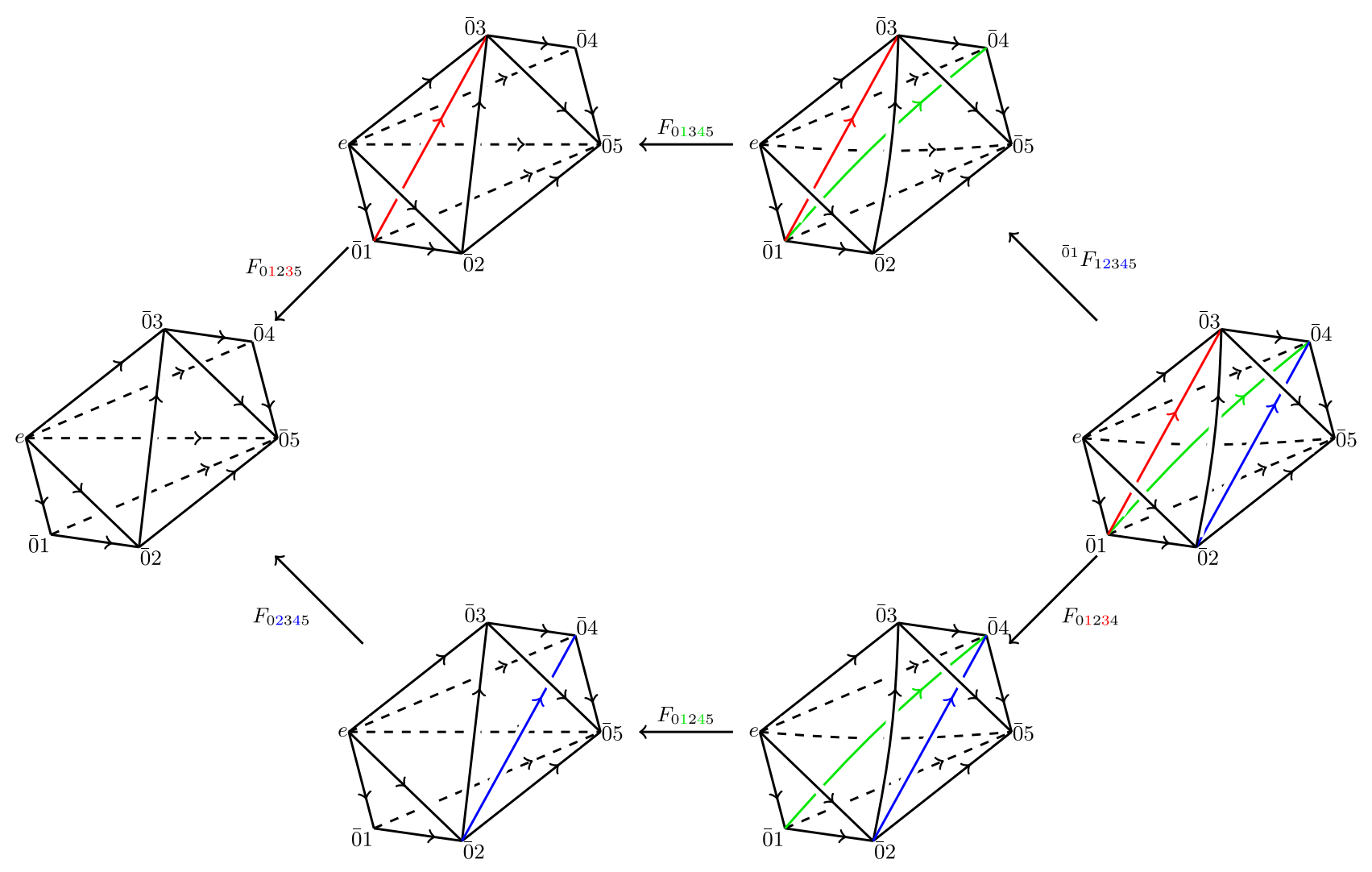

FIG. 11. Standard superfusion hexagon equation. Algebraically, this standard superhexagon condition corresponds to Eq. (213). The colored numbers $i$ and $j$ in the subscript of $F$ indicate that the link $\langle i j\rangle$ with the same color is added after this $F$ move. Since the group element label of the first vertex is $e \in G_{b}$, all the fermionic $F$ moves are standard except ${ }^{\overline{0} 1} F_{12345}$. We use a simpler notation $F_{i j k l m}=$ $F(e, \bar{i} j, \bar{j} k, \bar{k} l, \bar{l} m)$ for the standard $F$ move. We also omit all the fermion layers in the figure.

2-category. One should distinguish it from the hexagon equation of the braided tensor category, for the former is in the fusion level rather than the braided level. Our 3D FSPT states constructed correspond to some kind of pointed superfusion 2-categories with a given symmetry group.

It is enough to merely consider the standard superhexagon equations with first vertex label $e \in G_{b}$ as coherence conditions, because all other nonstandard ones can be obtained from it by a $U\left(g_{0}\right)$ symmetry action. The standard superhexagon equation is shown in Fig. 11 for the triangulation lattice and Fig. 12 for the dual lattice. Algebraically, we have the following equation:

$$
\begin{aligned}
F(e, \overline{0} 2, \overline{0} 3, \overline{0} 4, \overline{0} 5) \cdot F(e, \overline{0} 1, \overline{0} 2, \overline{0} 4, \overline{0} 5) \cdot F(e, \overline{0} 1, \overline{0} 2, \overline{0} 3, \overline{0} 4) & =F(e, \overline{0} 1, \overline{0} 2, \overline{0} 3, \overline{0} 5) \cdot F(e, \overline{0} 1, \overline{0} 3, \overline{0} 4, \overline{0} 5) \cdot F(\overline{0} 1, \overline{0} 2, \overline{0} 3, \overline{0} 4, \overline{0} 5) \\
& =F(e, \overline{0} 1, \overline{0} 2, \overline{0} 3, \overline{0} 5) \cdot F(e, \overline{0} 1, \overline{0} 3, \overline{0} 4, \overline{0} 5) \cdot{ }^{\circ} 1 F(e, \overline{1} 2, \overline{1} 3, \overline{1} 4, \overline{1} 5)
\end{aligned}
$$

where we again use $\bar{i} j$ to denote $g_{i}^{-1} g_{j}$. In the above equation, only the last $F$ symbol is nonstandard. It can be obtained from the standard one by a $U\left(g_{0}^{-1} g_{1}\right)$ symmetry action [see Eq. (203)].

Using the explicit expression of the standard $F$ move Eq. (189), we can unfold the standard superhexagon equation [Eq. (213)]. By eliminating all complex fermions and Majorana fermions, we can obtain a twisted cocycle equation for the inhomogeneous 3-cochain $\nu_{3}$ in the standard $F$ move. In general, the twisted cocycle equation reads

$$
d \nu_{4}=\mathcal{O}_{5}\left[n_{3}\right]
$$




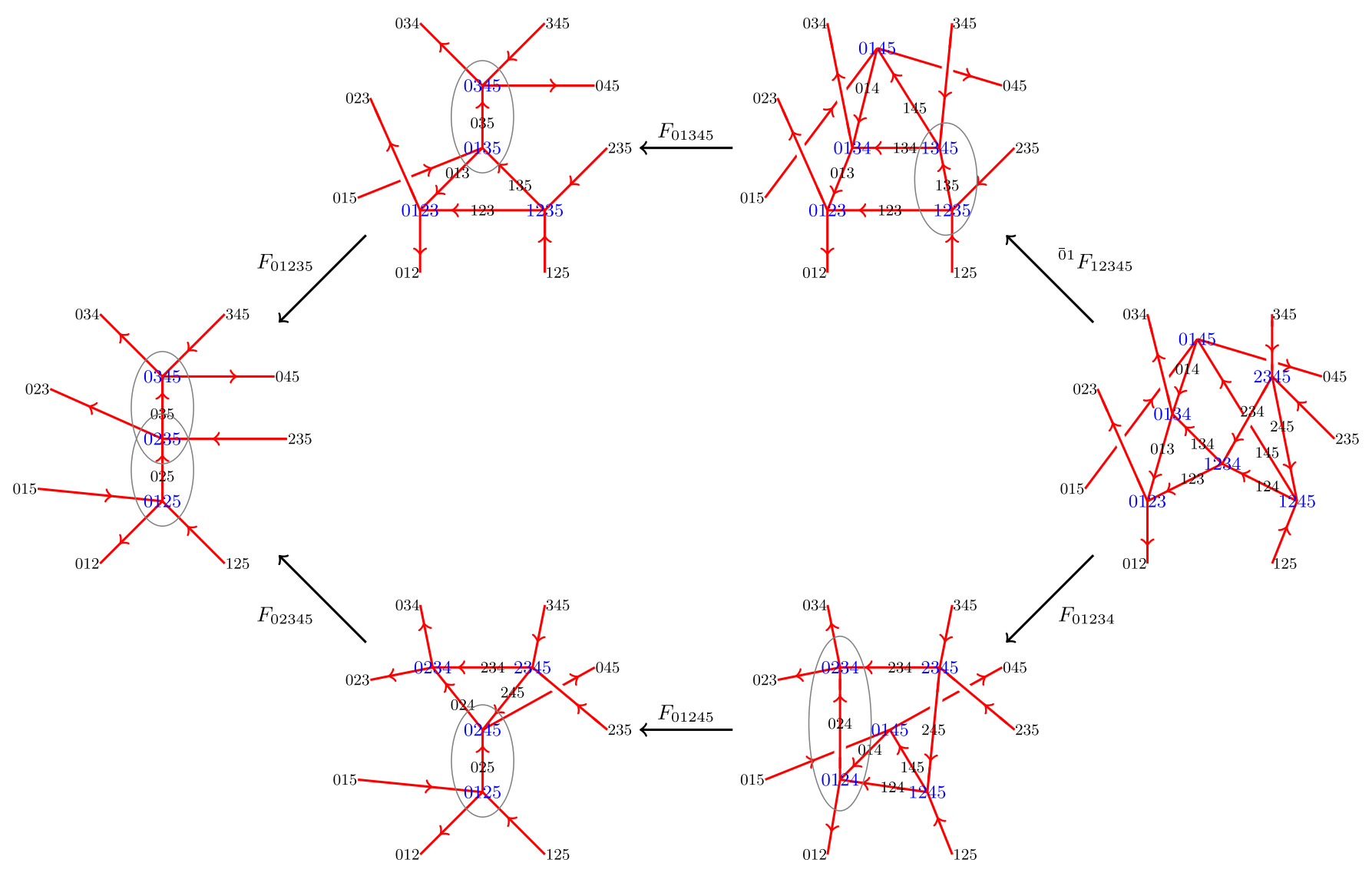

FIG. 12. Superfusion hexagon equation on the lattice dual to triangulation in Fig. 11. Each meeting point of four red links should be resolved to a small tetrahedron as in Fig. 9(b).

where $\mathcal{O}_{5}\left[n_{3}\right]$ is a functional of $n_{3}$ only (as well as $\omega_{2}$ and $s_{1}$ parametrizing the given symmetry group). The $n_{2}$ dependence of $\mathcal{O}_{5}$ is through $d n_{3}$ by Eq. (201). With nonzero $\omega_{2}$ and $s_{1}$, the obstruction function $\mathcal{O}_{5}\left[n_{3}\right]$ is more complicated than the special result for unitary $G_{f}=\mathbb{Z}_{2}^{f} \times G_{b}[53,62,63]$.

Similar to the $2 \mathrm{D}$ case, the obstruction function $\mathcal{O}_{5}\left[n_{3}\right]$ consists of four terms from general considerations:

$$
\mathcal{O}_{5}\left[n_{3}\right]=\mathcal{O}_{5}^{\mathrm{symm}}\left[n_{3}\right] \cdot \mathcal{O}_{5}^{c}\left[n_{3}\right] \cdot \mathcal{O}_{5}^{c \gamma}\left[d n_{3}\right] \cdot \mathcal{O}_{5}^{\gamma}\left[d n_{3}\right]
$$

The explicit expressions of these four terms are summarized, respectively, as

$$
\begin{aligned}
& \mathcal{O}_{5}^{\text {symm }}\left[n_{3}\right](012345)=(-1)^{\left(\omega_{2} \smile n_{3}+s_{1} \smile \alpha_{4}\right)(012345)+\omega_{2}(013) \alpha_{4}(12345)+\omega_{2}(012) \beta_{4}(12345)}, \\
& \mathcal{O}_{5}^{c}\left[n_{3}\right]=(-1)^{n_{3} \smile_{1} n_{3}+d n_{3} \smile_{2} n_{3}}, \\
& \mathcal{O}_{5}^{c \gamma}\left[d n_{3}\right]=(-1)^{d n_{3} \smile_{3} d n_{3}}, \\
& \left.\mathcal{O}_{5}^{\gamma}\left[d n_{3}\right](012345)\right|_{\beta_{4}=0}=(-1)^{d n_{3}(02345) d n_{3}(01235)+\omega_{2}(023)\left[d n_{3}(01245)+d n_{3}(01235)+d n_{3}(01234)\right]} \\
& \times i^{d n_{3}(01245) d n_{3}(01234)} \quad(\bmod 2) \times(-i)^{\left[d n_{3}(12345)+d n_{3}(02345)+d n_{3}(01345)\right] d n_{3}(01235)} \quad(\bmod 2) .
\end{aligned}
$$

We note that the expression of the last term $\mathcal{O}_{5}^{\gamma}\left[d n_{3}\right]$ is obtained under the assumption of $\beta_{4}=0$ [see Eq. (195)]. This assumption is true for $s_{1}=0$ (unitary $G_{b}$ ) or $n_{1}=0$. The calculation of generic $\mathcal{O}_{5}^{\gamma}$ with nonzero $\beta_{4}$ is much more complicated (but the procedures are the same), so we leave it for the future. By adding a coboundary $(-1)^{d\left(s_{1} \smile n_{3}+n_{3} \smile{ }_{3} d n_{3}\right)}$ to

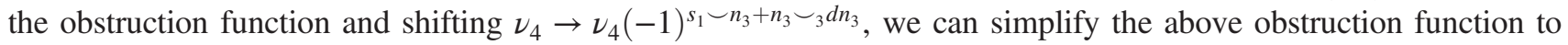




$$
\begin{aligned}
& \left.\mathcal{O}_{5}\left[n_{3}\right](012345)\right|_{\beta_{4}=0}=(-1)^{\left(\omega_{2} \smile n_{3}+n_{3} \smile_{1} n_{3}+n_{3} \smile_{2} d n_{3}\right)(012345)+\omega_{2}(013) d n_{3}(12345)} \\
& \times(-1)^{d n_{3}(02345) d n_{3}(01235)+\omega_{2}(023)\left[d n_{3}(01245)+d n_{3}(01235)+d n_{3}(01234)\right]} \\
& \times i^{d n_{3}(01245) d n_{3}(01234)}(\bmod 2) \times(-i)^{\left[d n_{3}(12345)+d n_{3}(02345)+d n_{3}(01345)\right] d n_{3}(01235)(\bmod 2)} .
\end{aligned}
$$

If we consider the special case of $\omega_{2}=s_{1}=0$, then we have $d n_{3}=n_{2} \smile n_{2}$ from Eq. (201). And the above obstruction reduces to the known result for $G_{f}=\mathbb{Z}_{2}^{f} \times G_{b}[53,62,63]$.

Before calculating the obstruction function $\mathcal{O}_{5}\left[n_{3}\right]$ in detail, we note that we check numerically that $\mathcal{O}_{5}\left[n_{3}\right]$ [Eq. (220)] satisfies $d \mathcal{O}_{5}=1$. It should be true, because the superhexagon equation [Eq. (213)] implies a one-higher-dimensional equation involving one more vertex.

\section{Calculations of obstruction function $\mathcal{O}_{5}\left[n_{3}\right]$}

In this subsection, we give explicit calculations of the four terms of the obstruction function $\mathcal{O}_{5}\left[n_{3}\right]$ in Eq. (215), with the assumption of $\beta_{4}=0$.

The first term $\mathcal{O}_{5}^{\text {symm }}\left[n_{3}\right]$ comes from the $U(\overline{0} 1)$ symmetry action on $F(e, \overline{1} 2, \overline{1} 3, \overline{1} 4, \overline{1} 5)$ in the last term of Eq. (213). Since the homogeneous $\nu_{4}$ of the nonstandard $F$ move is obtained from the standard one by a symmetry action [Eq. (210)], there is a sign difference $\mathcal{O}_{5}^{\text {symm }}$ between them. Using the replacement $\left(g_{0}, g_{1}, g_{2}, g_{3}, g_{4}\right) \rightarrow$ $\left(g_{0}^{-1} g_{1}, g_{0}^{-1} g_{2}, g_{0}^{-1} g_{3}, g_{0}^{-1} g_{4}, g_{0}^{-1} g_{5}\right)$ in the explicit expression [Eq. (211)], we have

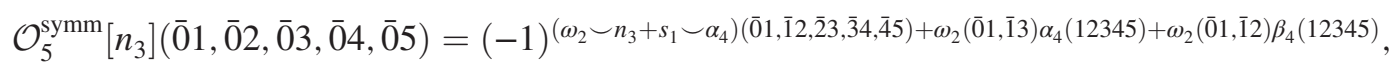

where $\alpha_{4}$ and $\beta_{4}$ are defined in Eqs. (194) and (195). This result is exactly Eq. (216) claimed above. If we consider the special case of $\beta_{4}=0$, the result is reduced to

$$
\left.\mathcal{O}_{5}^{\mathrm{symm}}\left[n_{3}\right](\overline{0} 1, \overline{0} 2, \overline{0} 3, \overline{0} 4, \overline{0} 5)\right|_{\beta_{4}=0}=(-1)^{\left(\omega_{2} \smile n_{3}+s_{1} \smile d n_{3}\right)(\overline{0} 1, \overline{1} 2, \overline{2} 3, \overline{3} 4, \overline{4} 5)+\omega_{2}(\overline{0} 1, \overline{1} 3) d n_{3}(12345)} .
$$

The second term $\mathcal{O}_{5}^{c}\left[n_{3}\right]$ is the complex fermion sign from reordering the fermion operators $\left(c_{i j k l}^{g_{0}^{-1} g_{i}}\right)^{n_{3}(i j k l)}$ in Eq. (213). To compare the complex fermion operators on the two sides of the superhexagon equation, we have to rearrange these operators. The reordering give us the complex fermion sign

$$
\begin{aligned}
\mathcal{O}_{5}^{c}\left[n_{3}\right](012345)= & (-1)^{\left(n_{3} \smile_{1} n_{3}+d n_{3} \smile_{2} n_{3}\right)(01234)} \\
= & (-1)^{n_{3}(0345) n_{3}(0123)+n_{3}(0145) n_{3}(1234)+n_{3}(0125) n_{3}(2345)} \\
& \cdot(-1)^{d n_{3}(01234) n_{3}(0145)+d n_{3}(02345) n_{3}(0125)+d n_{3}(01234) n_{3}(1245)+d n_{3}(01345) n_{3}(1235)+d n_{3}(01234) n_{3}(2345)+d n_{3}(01245) n_{3}(2345)} .
\end{aligned}
$$

This result is claimed in Eq. (217). It is a generalization of the sign $\left.\mathcal{O}_{5}\left[n_{3}\right]\right|_{S_{1}=\omega_{2}=n_{1}=n_{2}=0}=(-1)^{S q^{2}\left(n_{3}\right)}=(-1)^{n_{3} \smile_{1} n_{3}}$ in the special group supercohomology theory in Ref. [52]. The general group supercohomology theory for unitary $G_{f}=\mathbb{Z}_{2}^{f} \times G_{b}$ also has this complex fermion sign [53].

The third obstruction function term $\mathcal{O}_{4}^{c \gamma}\left[d n_{2}\right]$ originates from reordering the complex fermion and the Majorana fermions. It is also present in Ref. [53] and has the form

$$
\begin{aligned}
& \mathcal{O}_{5}^{c \gamma}\left[d n_{3}\right] \\
& =(-1)^{d n_{3} \smile_{3} d n_{3}} \\
& =(-1)^{d n_{3}(01245) d n_{3}(01234)+d n_{3}(01235) d n_{3}(01345)+d n_{3}(02345) d n_{3}(01234)+d n_{3}(02345) d n_{3}(01245)+d n_{3}(01235) d n_{3}(12345)+d n_{3}(01345) d n_{3}(12345)} .
\end{aligned}
$$

This result is exactly claimed previously in Eq. (218). This obstruction function is a functional of $d n_{3}$ (rather than $n_{3}$ directly), because the fermion parities of the $X$ operator and the complex fermion operator $c^{\dagger} c^{\dagger} c c$ depend only on $d n_{3}$.
In the rest of this subsection, we deal with the most complicated part $\mathcal{O}_{5}^{\gamma}\left[d n_{3}\right]$, with the assumption $\beta_{4}=0$. Similar to the Majorana fermion phase factor in Ref. [53], this obstruction function takes value in $\{ \pm 1, \pm i\}$. Since the 
presence of the dangling Majorana fermions in $X$ depends on $d n_{3}$, we expect that $\mathcal{O}_{5}^{\gamma}\left[d n_{3}\right]$ is a functional of $d n_{3}$ only.

Let us denote the six $X$ operators in the standard superhexagon equation [Eq. (213)] by $X_{12345}=$ $P_{2}\left(\gamma_{345 B}^{\overline{0} 3}\right)^{d n_{3}(12345)}, X_{02345}=P_{4}\left(\gamma_{345 B}^{\overline{0} 3}\right)^{d n_{3}(02345)}, X_{01345}=$ $P_{3}\left(\gamma_{345 B}^{\overline{0} 3}\right)^{d n_{3}(01345)}, X_{01245}=P_{5}\left(\gamma_{245 B}^{\overline{0} 2}\right)^{d n_{3}(01245)}, X_{01235}=$ $P_{4}\left(\gamma_{235 B}^{\overline{0} 2}\right)^{d n_{3}(01235)}$, and $X_{01234}=P_{6}\left(\gamma_{234 B}^{\overline{0} 2}\right)^{d n_{3}(01234)}$. Here, the operator $P_{i}$ is the Majorana pairing projection operator of the corresponding $i$ th figure $(1 \leq i \leq 6)$ in the superhexagon equation shown in Figs. 11 and 12. We use the convention that the rightmost figure is the first one with projection operator $P_{1}$, and the other five figures are counted counterclockwise. (Note that this labeling convention is different from Ref. [53].) We also use the simpler notations for the Majorana fermion operators

$$
\gamma_{i j k B}^{\overline{0} i}:=\gamma_{i j k B}^{g_{0}^{-1} g_{i}}
$$

Since the superhexagon equation involves only vertices from $\langle 0\rangle$ to $\langle 5\rangle$, we can simplify the argument of $d n_{3}$ as

$$
d n_{3}(\hat{i}):=d n_{3}(01 \ldots \hat{i} \ldots 45),
$$

where the number $i$ is removed in the argument. The obstruction function $\mathcal{O}_{5}^{\gamma}$ coming from Majorana fermions can be calculated from the $X$ operators as

$$
\begin{aligned}
\mathcal{O}_{5}^{\gamma}\left[d n_{3}\right] & =\left\langle X_{12345}^{\dagger} X_{01345}^{\dagger} X_{01235}^{\dagger} X_{02345} X_{01245} X_{01234}\right\rangle \\
& =\left\langle P_{1}\left(\gamma_{345 B}^{\overline{0} 3}\right)^{d n_{3}(\hat{0})} P_{2}\left(\gamma_{345 B}^{\overline{0} 3}\right)^{d n_{3}(\hat{2})} P_{3}\left(\gamma_{235 B}^{\overline{0} 2}\right)^{d n_{3}(\hat{4})} P_{4} P_{4}\left(\gamma_{345 B}^{\overline{0} 3}\right)^{d n_{3}(\hat{1})} P_{5}\left(\gamma_{245 B}^{\overline{0} 2}\right)^{d n_{3}(\hat{3})} P_{6}\left(\gamma_{234 B}^{\overline{0} 2}\right)^{d n_{3}(\hat{5})} P_{1}\right\rangle .
\end{aligned}
$$

The average is taken over the Majorana fermion pairing state of the rightmost figure (with projection operator $P_{1}$ ) in Figs. 11 and 12. We also insert $P_{1}$, which is 1 acting on the rightmost state, at the first and the last places of the operator string in Eq. (225).

We can calculate Eq. (225) separately for different Majorana fermion configurations specified by $n_{2}$. Among the six dangling Majorana fermions of the six $X$ operators in Eq. (225), only four of them are different:

$$
\left(\gamma_{345 B}^{\overline{0} 3}, \gamma_{245 B}^{\overline{0} 2}, \gamma_{235 B}^{\overline{0} 2}, \gamma_{234 B}^{\overline{0} 2}\right) \text {. }
$$

We can use the quadruple of their number,

$$
\left[d n_{3}(\hat{0})+d n_{3}(\hat{1})+d n_{3}(\hat{2}), d n_{3}(\hat{3}), d n_{3}(\hat{4}), d n_{3}(\hat{5})\right](\bmod 2),
$$

to indicate the presence or absence of the four Majorana fermions in Eq. (225), respectively. Each element of the quadruple corresponds to the Majorana fermion parity change of one or several $F$ moves. In total, there are $2^{4} / 2=$ 8 different possibilities for the Majorana fermion parity changes (see the first column in Table V), for the total Majorana fermion parity of the six $F$ moves should be even. For the eight different cases, we can calculate $\mathcal{O}_{5}^{\gamma}\left[d n_{3}\right]$ separately.

Let us consider the third case (the fourth row in Table V) as an example. The Majorana fermion parity change quadruple $\left[d n_{3}(\hat{0})+d n_{3}(\hat{1})+d n_{3}(\hat{2}), d n_{3}(\hat{3}), d n_{3}(\hat{4}), d n_{3}(\hat{5})\right](\bmod 2)$ corresponds to $(1,0,1,0)$. So the dangling Majorana fermions present in Eq. (225) are $\gamma_{345 B}^{\overline{0} 3}$ and $\gamma_{235 B}^{\overline{0} 2}$. We can expand the projection operators $P_{i}$ to Majorana fermion operators. Since $\gamma_{345 B}^{\overline{0} 3}$ and $\gamma_{235 B}^{\overline{0} 2}$ are paired inside the tetrahedron $\langle 2345\rangle$ of the lower right figure (with projection operator $P_{5}$ ) in Figs. 11 and 12 , we can consider only their pairing projection operator in $P_{5}$ [recall Eq. (98)]:

$$
P_{235 B, 345 B}^{\overline{0} 2, \overline{0} 3}=\frac{1}{2}\left[1-(-1)^{\omega_{2}(\overline{0} 2, \overline{2} 3)} i \gamma_{235 B}^{\overline{0} 2} \gamma_{345 B}^{\overline{0} 3}\right] .
$$

We can choose the second term in the above equation and all other projection operators $P_{i}$ with $i \neq 5$ to be 1 . Then, the obstruction function Eq. (225) can be expressed as

$$
\begin{aligned}
\mathcal{O}_{5}^{\gamma}[ & \left.d n_{3}\right]\left.(012345)\right|_{(1,0,1,0)} \\
= & \left\langle\left(\gamma_{345 B}^{\overline{0} 3}\right)^{d n_{3}(\hat{0})+d n_{3}(\hat{2})} \gamma_{235 B}^{\overline{0} 2}\left(\gamma_{345 B}^{\overline{0} 3}\right)^{d n_{3}(\hat{1})} P_{5}\right\rangle \\
= & \left\langle\left(\gamma_{345 B}^{\overline{0} 3}\right)^{d n_{3}(\hat{0})+d n_{3}(\hat{2})} \gamma_{235 B}^{\overline{0} 2}\left(\gamma_{345 B}^{\overline{0} 3}\right)^{d n_{3}(\hat{1})}\right. \\
& \left.\times(-1)^{\omega_{2}(\overline{0} 2, \overline{2} 3)}\left(-i \gamma_{235 B}^{\overline{0} 2} \gamma_{345 B}^{03}\right)\right\rangle \\
= & (-i)(-1)^{d n_{3}(\hat{1})+\omega_{2}(\overline{0} 2, \overline{2} 3)} .
\end{aligned}
$$

In such a way, Eq. (230) contains all Majorana fermions even times. After reordering these Majorana fermion operators, they all square to one. Finally, we obtain Eq. (231) as the obstruction function for this case.

We can similarly calculate $\mathcal{O}_{5}^{\gamma}\left[d n_{3}\right]$ for all eight cases of Majorana fermion parity changes. The information we need in the calculation is shown in Table V. Sometimes, we need not only $P_{5}$ but also $P_{4}$. And in $P_{4}$ or $P_{5}$, we may need several nontrivial Majorana pairing projection operators Eq. (98) (such as in the case of the last row in Table V). They are shown in the fourth and fifth columns in Table V. The final results of $\mathcal{O}_{5}^{\gamma}$ are shown in the last column in Table V. They can be summarized into two equivalent expressions as $\left(\mathcal{O}_{5}^{\gamma}\right.$ is a functional of $d n_{3}$ only) 
TABLE V. Calculations of $\mathcal{O}_{5}^{\gamma}\left[d n_{3}\right]$ for all possible Kitaev chain configurations in the superhexagon equation shown in Figs. 11 and 12. The first column is the Majorana fermion parity change quadruple $\left[d n_{3}(\hat{0})+d n_{3}(\hat{1})+d n_{3}(\hat{2}), d n_{3}(\hat{3}), d n_{3}(\hat{4}), d n_{3}(\hat{5})\right](\bmod 2)$. There are in total eight different cases. The second column is a simplified version of Eq. (225). The third and fourth columns are the Majorana pairing projection operators we use in the calculation. The last column is the final result of $\mathcal{O}_{5}^{\gamma}\left[d n_{3}\right]$, which can be summarized by Eq. (232).

\begin{tabular}{|c|c|c|c|c|}
\hline$P_{f}^{\gamma}$ changes & Expression of $\mathcal{O}_{5}^{\gamma}\left[d n_{3}\right]$ & $P_{4}$ & $P_{5}$ & $\mathcal{O}_{5}^{\gamma}\left[d n_{3}\right]$ \\
\hline$(0,0,0,0)$ & $\left\langle\left(\gamma_{345 B}^{\overline{0} 3}\right) d n_{3}(\hat{0})+d n_{3}(\hat{2})+d n_{3}(\hat{1})\right\rangle$ & 1 & 1 & 1 \\
\hline$(1,0,0,1)$ & $\left\langle\left(\gamma_{345 B}^{\overline{0} 3}\right)^{d n_{3}(\hat{0})+d n_{3}(\hat{2})} P_{4}\left(\gamma_{345 B}^{\overline{0} 3}\right)^{d n_{3}(\hat{1})} P_{5} \gamma_{234 B}^{\overline{0} 2}\right\rangle$ & $P_{234 A, 234 B}^{\overline{0} 2, \overline{0} 2}$ & $P_{345 B, 234 A}^{\overline{0} 3, \overline{0} 2}$ & $(-1)^{\omega_{2}(\overline{0} 2, \overline{2} 3)}$ \\
\hline$(1,0,1,0)$ & $\left\langle\left(\gamma_{345 B}^{\overline{0} 3}\right)^{d n_{3}(\hat{0})+d n_{3}(\hat{2})} \gamma_{235 B}^{\overline{0} 2}\left(\gamma_{345 B}^{\overline{0} 3}\right)^{d n_{3}(\hat{1})} P_{5}\right\rangle$ & 1 & $P_{235 B, 345 B}^{\overline{0} 2, \overline{0} 3}$ & $(-i)(-1)^{d n_{3}(\hat{1})+\omega_{2}(\overline{0} 2, \overline{2} 3)}$ \\
\hline$(0,0,1,1)$ & $\left\langle\left(\gamma_{345 B}^{\overline{0} 3}\right)^{d n_{3}(\hat{0})+d n_{3}(\hat{2})} \gamma_{235 B}^{\overline{0} 2} P_{4}\left(\gamma_{345 B}^{\overline{0} 3}\right)^{d n_{3}(\hat{1})} P_{5} \gamma_{234 B}^{\overline{0} 2}\right\rangle$ & $P_{234 A, 234 B}^{\overline{0} 2, \overline{0} 2}$ & $P_{235 B, 234 A}^{\overline{0} 2, \overline{2} 2}$ & $(-1)^{d n_{3}(\hat{1})}$ \\
\hline$(1,1,0,0)$ & $\left\langle\left(\gamma_{345 B}^{\overline{0} 3}\right)^{d n_{3}(\hat{0})+d n_{3}(\hat{2})} P_{4}\left(\gamma_{345 B}^{\overline{0} 3}\right)^{d n_{3}(\hat{1})} P_{5} \gamma_{245 B}^{\overline{0} 2}\right\rangle$ & $P_{245 A, 245 B}^{\overline{0} 2, \overline{0} 2}$ & $P_{345 B, 245 A}^{\overline{0} 3, \overline{2} 2}$ & $(-1)^{\omega_{2}(\overline{0} 2, \overline{2} 3)}$ \\
\hline$(0,1,0,1)$ & $\left\langle\left(\gamma_{345 B}^{\overline{0} 3}\right)^{d n_{3}(\hat{0})+d n_{3}(\hat{2})} P_{4}\left(\gamma_{345 B}^{\overline{0} 3}\right)^{d n_{3}(\hat{1})} P_{5} \gamma_{245 B}^{\overline{0} 2} \gamma_{234 B}^{\overline{0} 2}\right\rangle$ & $\begin{array}{l}P_{234 A, 234 B}^{\overline{0} 2, \overline{0}} \\
P_{245 A, 245 B}^{\overline{0} 2, \overline{0} 2}\end{array}$ & $P_{234 A, 245 A}^{\overline{0} 2, \overline{0} 2}$ & $i$ \\
\hline$(0,1,1,0)$ & $\left\langle\left(\gamma_{345 B}^{\overline{0} 3}\right)^{d n_{3}(\hat{0})+d n_{3}(\hat{2})} \gamma_{235 B}^{\overline{0} 2} P_{4}\left(\gamma_{345 B}^{\overline{0} 3}\right)^{d n_{3}(\hat{1})} P_{5} \gamma_{245 B}^{\overline{0} 2}\right\rangle$ & $P_{245 A, 245 B}^{\overline{0} 2, \overline{0} 2}$ & $P_{235 B, 245 A}^{\overline{0} 2, \overline{0} 2}$ & $(-1)^{d n_{3}(\hat{1})}$ \\
\hline$(1,1,1,1)$ & $\left\langle\left(\gamma_{345 B}^{\overline{0} 3}\right)^{d n_{3}(\hat{0})+d n_{3}(\hat{2})} \gamma_{235 B}^{\overline{0} 2} P_{4}\left(\gamma_{345 B}^{\overline{0} 3}\right)^{d n_{3}(\hat{1})} P_{5} \gamma_{245 B}^{\overline{0} 2} \gamma_{234 B}^{\overline{0} 2}\right\rangle$ & $\begin{array}{l}P_{234 A, 234 B}^{\overline{0} 2, \overline{0} 2} \\
P_{245 A, 245 B}^{\overline{0} 2, \overline{0} 2} \\
\end{array}$ & $\begin{array}{l}P_{235 B, 345 B}^{\overline{0} 2, \overline{0} 3} \\
P_{234 A, 245 A}^{\overline{0} 2, \overline{0} 2} \\
\end{array}$ & $(-1)^{d n_{3}(\hat{1})+\omega_{2}(\overline{0} 2, \overline{2} 3)}$ \\
\hline
\end{tabular}

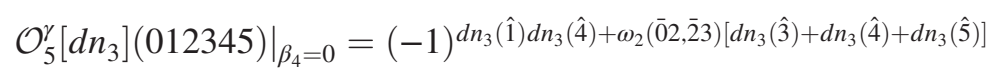

$$
\begin{aligned}
& \times i^{d n_{3}(\hat{3})\left[1-d n_{3}(\hat{4})\right] d n_{3}(\hat{5})} \quad(\bmod 2) \times(-i)^{\left[1-d n_{3}(\hat{3})\right] d n_{3}(\hat{4})\left[1-d n_{3}(\hat{5})\right]} \quad(\bmod 2)
\end{aligned}
$$

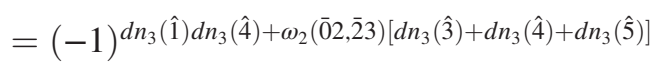

$$
\begin{aligned}
& \times i^{d n_{3}(\hat{3}) d n_{3}(\hat{5})} \quad(\bmod 2) \times(-i)^{\left[d n_{3}(\hat{0})+d n_{3}(\hat{1})+d n_{3}(\hat{2})\right] d n_{3}(\hat{4}) \quad(\bmod 2)} .
\end{aligned}
$$

Note that the $d n_{3}$ terms in the exponent of $( \pm i)$ should be understood as taking mod 2 values (can be only 0 or 1 ). They correspond to the third and sixth cases in Table V. The term $(-1)^{d n_{3}(\hat{1})}$ appears in the last column in Table $\mathrm{V}$ if and only if $d n_{3}(\hat{4})=1$. And the term $(-1)^{\omega_{2}(\overline{0} 2, \overline{2} 3)}$ appears whenever $d n_{3}(\hat{3})+d n_{3}(\hat{4})+d n_{3}(\hat{5})=1(\bmod 2)$. That is the origin of the $(-1)$ terms in Eq. (232). Note that the exponent of $\pm i$ for the first (second) expression of $\mathcal{O}_{5}^{\gamma}\left[d n_{3}\right]$ is a cubic (quadratic) $\mathbb{Z}_{2}$-valued function of $n_{3}$. The expression of $\mathcal{O}_{5}^{\gamma}\left[d n_{3}\right]$ is the same as the claimed Eq. (219).

If we consider the unitary symmetry group $G_{f}=\mathbb{Z}_{2}^{f} \times$ $G_{b} \quad\left(\omega_{2}=s_{1}=0\right)$, the Majorana obstruction function Eq. (232) is reduced to an expression as a functional of $n_{2}^{2}$ (because of $d n_{3}=n_{2} \smile n_{2}$ in this case). Although the expression is different from the result in Ref. [53] in appearance, one can show that they are exactly the same after some calculations. It is also the same as the $(3+1) \mathrm{D}$ spin cobordism result $[62,63]$.

\section{E. Boundary ASPT states}

In the above discussions in this section, we construct 3D FSPT states by decorating several layers of fermion modes to the BSPT states. The decorations of Kitaev chains and complex fermions are specified by two $\mathbb{Z}_{2}$-valued cochains $n_{2}$ and $n_{3}$.

In this section, we show that some of the $n_{2}$ and $n_{3}$ data, in fact, correspond to FSPT states belonging to the trivial FSPT phase. This data trivialization can be understood by investigating the boundary ASPT states on the 2D boundary of the 3D FSPT bulk.

\section{Boundary ASPT states in $\Gamma^{2}$ with $p+i p$ superconductors}

Similar to the 2D case, we show in this section that the 3D FSPT data $n_{2}=\omega_{2}$ are trivialized by the boundary ASPT state. The 2D boundary is, in fact, a state with (one layer) $p+i p$ chiral superconductor decorations.

Since there is no fixed-point wave function construction for 2D $p+i p$ superconductor on a discrete lattice [80], its decoration is very different from the Kitaev chains and complex fermions. In the boundary ASPT state construction, we put $p+i p$ superconductor domains around each vertex $i$ of the triangulation lattice and use symmetric mass terms to gap out the edge modes and glue them together. In the following, we first discuss the procedure of decorating 
$p+i p$ superconductors. Then, we propose a field theory description of symmetrically gapping out the chiral Majorana modes along the edge.

The detailed decorations are as follows. For an arbitrary triangulation of the 2D boundary of a 3D FSPT state, we put $p+i p$ superconductors on a disk centered at each vertex $i$. The 2D disks of the superconductors are always gapped. And on the boundary, there are right-moving chiral Majorana modes [see the green arrows in Eq. (233)]. We denote these chiral Majorana modes by $\psi_{i, R}^{g_{i}}$. A picture of the chiral Majorana modes in a triangle of the 2D boundary of 3D FSPT bulk is

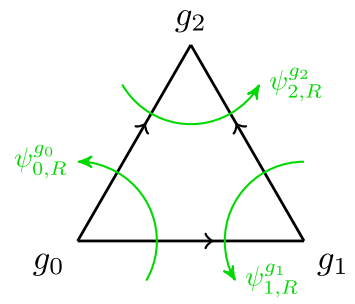

The decorated state should be symmetric under $G_{b}$ action. If the chiral Majorana modes around vertex $i$ with $g_{i}=e$ are $\psi_{i, R}^{e}$, then, for generic $g_{i} \in G_{b}$, the decorated $p+i p$ superconductors should have chiral Majorana modes $\psi_{i, g_{i}(R) ; \alpha}^{g_{i}}$ according to the symmetry transformation rules:

$$
\begin{gathered}
U(g) \psi_{i, R}^{\sigma} U(g)^{\dagger}=(-1)^{\omega_{2}(g, \sigma)} \psi_{i, g(R)}^{g \sigma}, \\
U(g) \psi_{i, L}^{\sigma} U(g)^{\dagger}=(-1)^{\omega_{2}(g, \sigma)+s_{1}(g)} \psi_{i, g(L)}^{g \sigma} .
\end{gathered}
$$

Here, we denote $g_{i}(R)=R$ if $s_{1}\left(g_{i}\right)=0$ ( $g_{i}$ is unitary) and $g_{i}(R)=L$ if $s_{1}\left(g_{i}\right)=1$ ( $g_{i}$ is antiunitary). The physical meaning is that the unitary symmetry $U(g)$ changes only the group element label of chiral modes from $g_{i}$ to $g g_{i}$. However, time-reversal symmetry transforms $p+i p$ superconductors to $p-i p$ superconductors, and vice versa. So the right-moving and left-moving chiral Majorana modes on the edge are switched.

The next step in our construction is to gap out all the chiral Majorana modes to obtain a fully gapped boundary state. Consider the interface between two $p+i p$ domains labeled by $g_{0}$ and $g_{1}$ shown below:

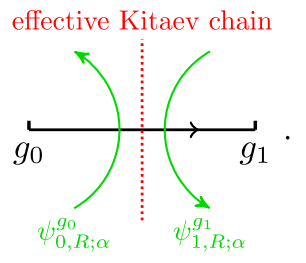

There is a necessary condition for gapping out the chiral Majorana modes $\psi_{0, R / L}^{g_{0}}$ and $\psi_{1, R / L}^{g_{1}}$ shown by green arrows above: The number of net chiral modes along the direction dual to $\langle 01\rangle$ is zero. If $s_{1}\left(g_{0}^{-1} g_{1}\right)=1$, then one of the two right-moving chiral Majorana modes is reversed. There are two chiral modes moving in the same direction along the link dual to $\langle 01\rangle$. This result is illegal, for we want the boundary to be a gapped state. So we conclude that the boundary ASPT state with $p+i p$ superconductors is impossible if there are some antiunitary symmetries in $G_{b}$. In the following discussions of the ASPT state with $p+i p$ superconductors, we always assume $G_{b}$ is unitary.

Let us introduce explicitly the symmetric mass terms to gap out the chiral Majorana modes. Similar to the previous discussions, we can first assume the group element labels of link $\langle 01\rangle$ are $e$ and $g_{0}^{-1} g_{1}$. The standard mass terms along the edge dual to link $\langle 01\rangle$ are

$$
H_{\mathrm{std}}^{\langle 01\rangle}=i m \int d x \psi_{0, R}^{e}(x) \psi_{1, R}^{g_{0}^{-1} g_{1}}(x)
$$

The nonstandard mass terms are obtained from the above standard ones by a symmetry action:

$$
\begin{aligned}
H_{\text {mass }}^{\langle 01\rangle} & =U\left(g_{0}\right) H_{\text {std }}^{\langle 01\rangle} U\left(g_{0}\right)^{\dagger} \\
& =(-1)^{\omega_{2}\left(g_{0}, g_{0}^{-1} g_{1}\right)} i m \int d x \psi_{0, R}^{g_{0}}(x) \psi_{1, R}^{g_{1}}(x),
\end{aligned}
$$

where we use the symmetry transformation rule Eq. (234) of Majorana modes. Note that there is no minus sign from the imaginary unit $i$, for $G_{b}$ should be unitary according to the previous discussions. The mass terms constructed above are, of course, $G_{b}$ symmetric, since they are all obtained from the standard mass terms by a symmetry action.

It is well known that changing the sign of the mass $m$ of two counterpropagating chiral Majorana modes induces a phase transition from a nontopological superconductor to a topological superconductor [81]. If the resulting gapped edge is a 1D topological superconductor, there is effectively a Kitaev chain going along the direction of the chiral modes [see the dashed red line in Eq. (236)]. We can assume the standard mass terms Eq. (237) with $m>0$ correspond to the trivial gapped phase. Then, the true mass terms Eq. (238) induce effective Kitaev chains going through the link $\langle 01\rangle$. The number of effective Kitaev chains equals the number of negative mass terms:

$$
\omega_{2}\left(g_{0}, g_{0}^{-1} g_{1}\right)
$$

If we consider the triangle $\langle 012\rangle$ with mass terms $H_{\text {mass }}^{\langle 12\rangle}$, $H_{\text {mass }}^{\langle 02\rangle}$, and $H_{\text {mass }}^{\langle 01\rangle}$ on the boundary, the (mod 2$)$ number of effective Kitaev chains going through the three links is 


$$
\begin{aligned}
\omega_{2}\left(g_{0}^{-1} g_{1}, g_{1}^{-1} g_{2}\right)= & \omega_{2}\left(g_{1}, g_{1}^{-1} g_{2}\right)+\omega_{2}\left(g_{0}, g_{0}^{-1} g_{2}\right) \\
& +\omega_{2}\left(g_{0}, g_{0}^{-1} g_{1}\right),
\end{aligned}
$$

where we use $\left(d \omega_{2}\right)\left(g_{0}, g_{0}^{-1} g_{1}, g_{1}^{-1} g_{2}\right)=0(\bmod 2)$.

If $\omega_{2}$ is nontrivial in Eq. (240), there may be an odd number of Kitaev chains going into the triangle $\langle 012\rangle$. Since our state is on the boundary of a 3D FSPT bulk, we can connect the Kitaev chain to the 3D bulk FSPT state [see Eq. (173)]. The number of Kitaev chains going through a triangle of a 3D FSPT state is exactly the $n_{2}$ data which are discussed in detail in Sec. VI B 1. So we have $n_{2}=\omega_{2}$ to have a gapped state (including both the boundary and the bulk). Since we construct a gapped symmetric (ASPT) state without topological order on the boundary of the 3D FSPT state, we conclude that the bulk FSPT with $n_{2}=\omega_{2}$ is trivial. This result is the origin of the trivialization group

$\Gamma^{2}=\left\{\omega_{2} \smile n_{0} \in H^{2}\left(G_{b}, \mathbb{Z}_{2}\right) \mid n_{0} \in H^{0}\left(G_{b}, \mathbb{Z}_{T}\right)\right\}$

for 3D FSPT phases claimed in Eq. (19). We note that the $p+i p$ superconductor is incompatible with time-reversal symmetry. So there is no $\Gamma^{2}$ trivialization if $G_{b}$ contains antiunitary symmetry. In other words, Eq. (241) is equivalent to

$\Gamma^{2}= \begin{cases}\left\{\omega_{2} \in H^{2}\left(G_{b}, \mathbb{Z}_{2}\right)\right\}, & G_{b} \text { is unitary, } \\ 0, & G_{b} \text { contains antiunitary }\end{cases}$

because $H^{0}\left(G_{b}, \mathbb{Z}_{T}\right)=\mathbb{Z} \quad$ if $\quad G_{b}$ is unitary and $H^{0}\left(G_{b}, \mathbb{Z}_{T}\right)=0$ if $G_{b}$ contains antiunitary symmetries.

\section{Boundary ASPT states in $\Gamma^{3}$ with $p+i p$ superconductors}

The ASPT state in Eq. (241) is realized as one layer of a $2 \mathrm{D} p+i p$ superconductor $\left[n_{0}=1 \in H^{0}\left(G_{b}, \mathbb{Z}_{T}\right)\right]$ on the boundary of 3D FSPT states with $n_{2}=\omega_{2}$ Majorana chain decorations. The fluctuating Majorana chains (the $n_{2}$ data) in the 3D bulk become the effective Majorana chains along the $1 \mathrm{D}$ domain walls of $2 \mathrm{D}$ boundary $p+i p$ superconductors. Therefore, there is a gapped symmetric boundary without topological order, and the 3D bulk is trivialized.

In this section, we consider the ASPT state with two layers of 2D $p+i p$ superconductor decoration $\left[n_{0}=2 \in H^{0}\left(G_{b}, \mathbb{Z}_{T}\right)\right]$. So, according to Eq. (241), there is no $\Gamma^{2}$ trivialization. However, we show below that there is a $\Gamma^{3}$ trivialization of the 3D FSPT state with $n_{3}=$ $\omega_{2} \smile_{1} \omega_{2}$ complex fermion decorations. This result is related to the fact that the $F$ move of $n_{0}=2 p+i p$ superconductors on the 2D boundary changes fermion parity by $\Delta P_{f}^{\psi}(F)=(-1)^{\omega_{2} \smile_{1} \omega_{2}}$. This result is similar to the $\Gamma^{3}$ trivialization related to $n_{1}$ (2D boundary Majorana chain decorations) discussed in the next section.
The setup of the $n_{0}=2$ ASPT state is similar to the state discussed in Sec. VIE1. Near the vertex $i$ of the space triangulation, we put a disk of two layers of $p+i p$ superconductors labeled by $g_{i}$. Along the boundary, we have two chiral Majorana modes indicated by green curves in, for example, Eqs. (233) and (236). After adding symmetric mass terms along the line dual to link $\langle i j\rangle$, we can gap out the chiral Majorana modes. If the sign of mass $(-1)^{\omega_{2}\left(g_{i}, g_{i}^{-1} g_{j}\right)}$ [see the discussions above Eq. (239)] is negative, there are $n_{0}=2$ effective Majorana chains going through the link $\langle i j\rangle$. Since we want to analyze the fermion parities, we have to pair up the Majorana fermions in the effective Majorana chains. If $\omega_{2}\left(g_{i}, g_{i}^{-1} g_{j}\right)=1$, there are $n_{0}=2$ effective Majorana chains, leaving two Majorana fermions on each side of link $\langle i j\rangle$. We can pair them up as shown in Fig. 13(b) (always from $\alpha=1$ to $\alpha=2$ on each side). Note that the vacuum pairings for $\omega_{2}\left(g_{i}, g_{i}^{-1} g_{j}\right)=0$ are the standard $A$ to $B$ pairings [see Fig. 13(a)]. So the fermion parity of the nontrivial pairings is always odd (compared to the vacuum pairings), as the small loop with length four is non-Kasteleyn oriented.

Now we can consider the $p+i p$ superconductor configurations in one triangle $\langle 012\rangle$. The three mass term signs $\omega_{2}\left(g_{i}, g_{i}^{-1} g_{j}\right)$ for the three links are independent of each other. So we have in total $2^{3}=8$ configurations, which can be divided into four cases. (i) All three $\omega_{2}$ 's are zero. Then all the effective Majorana fermions near the three links are in the vacuum pairings shown in Fig. 13(a). And the fermion parity of this configuration is $P_{f}^{\psi}=+1$. (ii) One of the three $\omega_{2}$ 's is one [see Fig. 14(a) of $\omega_{2}\left(g_{1}, g_{1}^{-1} g_{2}\right)=1$, for example]. Then there is only one length-4 loop with non-Kasteleyn orientation. And the fermion parity is $P_{f}^{\psi}=(-1)^{1}=-1$. (iii) Two of the three $\omega_{2}$ 's are one [see Fig. 14(b) of $\omega_{2}\left(g_{0}, g_{0}^{-1} g_{2}\right)=\omega_{2}\left(g_{1}, g_{1}^{-1} g_{2}\right)=1$, for example]. We connect the effective Majorana chains going through the two links (according to the Kasteleyn orientation rules in Fig. 2). So the total fermion parity of this configuration is $P_{f}^{\psi}=(-1)^{3}=-1$. (iv) All three $\omega_{2}$ 's are one [see Fig. 14(c), for example]. We again connect the effective Majorana chains going through two (arbitrary)

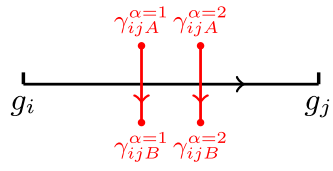

(a)

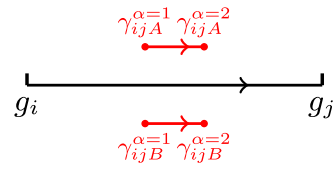

(b)
FIG. 13. There are $n_{0}=2$ effective Majorana chains $(\alpha=1,2)$ going through link $\langle i j\rangle$ iff $\omega_{2}\left(g_{i}, g_{i}^{-1} g_{j}\right)=1$. The vacuum and nontrivial pairing directions are shown in (a) and (b), respectively. We note that $\alpha=1,2$ effective Majorana chains should be understood as the stacking in the direction perpendicular to the paper. They are not related to the branching structure (black arrows). 


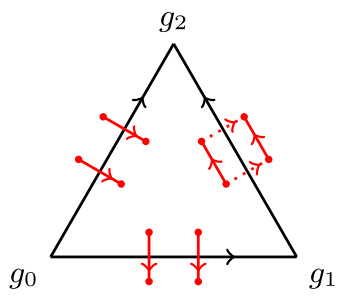

(a)

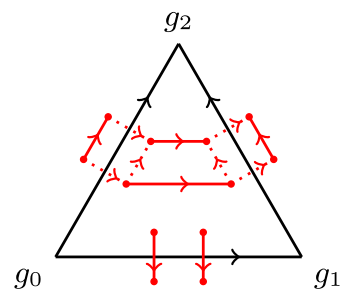

(b)

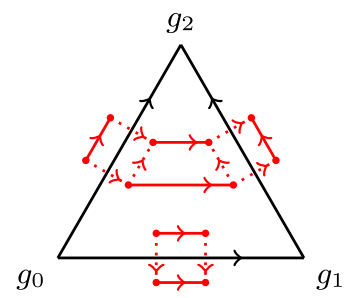

(c)

FIG. 14. Different effective Majorana chain configurations and their total fermion parities $P_{f}^{\psi}$. The fermion parity can be easily calculated by counting the number of length-4 (non-Kasteleyn-oriented) loops in the figures. (a) $\omega_{2}\left(g_{i}, g_{i}^{-1} g_{j}\right)=1$ for one link with fermion parity $P_{f}^{\psi}=(-1)^{1}$. (b) $\omega_{2}\left(g_{i}, g_{i}^{-1} g_{j}\right)=1$ for two links with fermion parity $P_{f}^{\psi}=(-1)^{3}$. (c) $\omega_{2}\left(g_{i}, g_{i}^{-1} g_{j}\right)=1$ for three links with fermion parity $P_{f}^{\psi}=(-1)^{4}$.

links [for example, links $\langle 02\rangle$ and $\langle 12\rangle$ in Fig. 14(c)] and leave the third link Majorana chain unchanged. Then, the total fermion parity of this configuration is $P_{f}^{\psi}=(-1)^{4}=+1$. The total fermion parity is independent of the choice we make to connect the effective Majorana chains. We can choose arbitrary two links of the triangle and connect the Majorana fermions through them. This result is why we choose the conventions in Fig. 13. For all the configurations, one can check easily that the fermion parity can be summarized as

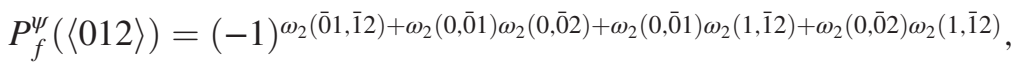

where we use $\left(d \omega_{2}\right)(0, \overline{0} 1, \overline{1} 2)=\omega_{2}(\overline{0} 1, \overline{1} 2)+\omega_{2}(1, \overline{1} 2)+$ $\omega_{2}(0, \overline{0} 2)+\omega_{2}(0, \overline{0} 1)=0(\bmod 2)$.

With the fermion parity formula for one triangle, we can derive the fermion parity change of the standard $F$ move Eq. (111). Since all $\omega_{2}\left(g_{i}, g_{i}^{-1} g_{j}\right)$ for link $\langle i j\rangle$ are, in general, independent of each other, there are in total $2^{6}=$ 64 possible configurations for the $F$ move. The fermion parity change of the $F$ move is obtained from the fermion parities of the four relevant triangles on the two sides. However, the small dimer cover loop crossing links $\langle 02\rangle$ and $\langle 13\rangle$ are counted twice by the two adjacent triangles. Therefore, the fermion parity change of the $F$ move is the product of four triangle fermion parities [see Eq. (243) for triangle $\langle 012\rangle]$ with a modification factor $(-1)^{\omega_{2}(0, \overline{0} 2)+\omega_{2}(1, \overline{1} 3)}$. After some tedious calculations, one can show that the final result is the cup-1 product of $\omega_{2}$ :

$$
\begin{aligned}
& \Delta P_{f}^{\psi}(F)=P_{f}^{\psi}(\langle 012\rangle) \cdot P_{f}^{\psi}(\langle 023\rangle) \cdot P_{f}^{\psi}(\langle 013\rangle) \\
& \cdot P_{f}^{\psi}(\langle 123\rangle) \cdot(-1)^{\omega_{2}(0, \overline{0} 2)+\omega_{2}(1, \overline{1} 3)}
\end{aligned}
$$

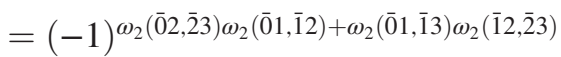

$$
\begin{aligned}
& =(-1)^{\left(\omega_{2} \smile_{1} \omega_{2}\right)(\overline{0} 1, \overline{1} 2, \overline{2} 3)} \text {. }
\end{aligned}
$$

If the exponent in Eq. (244) is a nontrivial cocycle in $H^{3}\left(G_{b}, \mathbb{Z}_{2}\right)$, then the $2 \mathrm{D} F$ move necessarily breaks the fermion parity of the $p+i p$ superconductor system. However, if we introduce a 3D FSPT bulk with complex fermion decoration $n_{3}=\omega_{2} \smile_{1} \omega_{2}$, the total fermion parity is preserved. This preservation is simply because the $2 \mathrm{D}$ boundary $F$ move also changes the bulk complex fermion number by $n_{3}\left(g_{0}, g_{1}, g_{2}, g_{3}\right)$. Therefore, two layers of $p+i p$ superconductors can be viewed as a 2D ASPT state which trivializes the 3D FSPT state with complex fermion decoration $n_{3}=\omega_{2} \smile_{1} \omega_{2}$. So we have a trivialization group $\Gamma^{3}$ as

$\Gamma^{3} \supset\left\{\left(\omega_{2} \smile_{1} \omega_{2}\right)\left\lfloor n_{0} / 2\right\rfloor \in H^{3}\left(G_{b}, \mathbb{Z}_{2}\right) \mid n_{0} \in H^{0}\left(G_{b}, \mathbb{Z}_{T}\right)\right\}$.

Note that we introduce $n_{0} \in H^{0}\left(G_{b}, \mathbb{Z}_{T}\right)$ in the above expression. Only unitary $G_{b}$ is compatible with $p+i p$ chiral superconductors. And odd $n_{0}$ (with nontrivial $\omega_{2}$ ) already leads to nontrivial trivialization group $\Gamma^{2}$ Eq. (241) in a lower level. Only $n_{0}=2$ produces nontrivial $\Gamma^{3}$ and a nontrivial ASPT state on the boundary of 3D FSPT with $n_{3}=\omega_{2} \smile_{1} \omega_{2}$. An explicit example of this $\Gamma^{3}$ trivialization Eq. (245) with $n_{0}=2$ is the FSPT state with $G_{f}=Q_{8}^{f}$ (see Appendix E 3).

\section{Boundary ASPT states in $\Gamma^{3}$ with Kitaev chains}

There is another layer of boundary ASPT state for the 3D FSPT state. This boundary ASPT state has Kitaev chain decorations and trivializes the complex fermion decoration data

$$
n_{3}=\omega_{2} \smile n_{1}+s_{1} \smile n_{1} \smile n_{1},
$$

for some $n_{1} \in H^{1}\left(G_{b}, \mathbb{Z}_{2}\right)$. The second part $s_{1} \smile n_{1} \smile n_{1}$ is discussed in detail in Ref. [66]. It is used to construct a 2D 
ASPT state on the boundary of 3D FSPT state with timereversal symmetry $T^{2}=1$.

The generic boundary ASPT state with Kitaev chains can be constructed similar to the 2D FSPT state with Kitaev chain decorations in Sec. VB 1. We put (at most) one Kitaev chain along the (red) dual link on the 2D boundary according to the decoration data $n_{1} \in H^{1}\left(G_{b}, \mathbb{Z}_{2}\right)$. The fermion parity change of the Majorana fermions under the boundary 2D $F$ move is again given by [see Eq. (117)]

$$
\Delta P_{f}^{\gamma}(F)=(-1)^{\left(\omega_{2} \smile n_{1}+s_{1} \smile n_{1} \smile n_{1}\right)\left(g_{0}^{-1} g_{1}, g_{1}^{-1} g_{2}, g_{2}^{-1} g_{3}\right)} .
$$

If the exponent of $(-1)$ is not a $\mathbb{Z}_{2}$-valued coboundary, we cannot preserve the boundary fermion parity even if we introduce complex fermion decorations on the boundary. However, since the ASPT state is on the boundary of a 3D FSPT bulk, we can simply choose the bulk complex fermion decoration data $n_{3}$ with Eq. (246). Therefore, the total system (including both the boundary and the bulk) has definite total fermion parity.

Since we construct a gapped symmetric ASPT state without topological order on the boundary of the 3D FSPT state, we conclude that the bulk FSPT with $n_{3}=\omega_{2} \smile n_{1}+$ $s_{1} \smile n_{1} \smile n_{1}$ is trivialized. This conclusion is the origin of the $n_{1}$ part of the trivialization group $\Gamma^{3}$ :

$$
\begin{aligned}
\Gamma^{3} & \supset\left\{\omega_{2} \smile n_{1}+s_{1} \smile n_{1} \smile n_{1} \in H^{3}\left(G_{b}, \mathbb{Z}_{2}\right) \mid n_{1}\right. \\
& \left.\in H^{1}\left(G_{b}, \mathbb{Z}_{2}\right)\right\} .
\end{aligned}
$$

Combine it with Eq. (245), and we obtain the trivialization group

$$
\begin{aligned}
\Gamma^{3} & =\left\{\omega_{2} \smile_{1}+s_{1} \smile n_{1} \smile_{1}+\left(\omega_{2} \smile_{1} \omega_{2}\right)\left\lfloor n_{0} / 2\right\rfloor\right. \\
& \left.\in H^{3}\left(G_{b}, \mathbb{Z}_{2}\right) \mid n_{1} \in H^{1}\left(G_{b}, \mathbb{Z}_{2}\right), n_{0} \in H^{0}\left(G_{b}, \mathbb{Z}_{T}\right)\right\},
\end{aligned}
$$

which is claimed in Eq. (19).

\section{F. An additional layer of $\boldsymbol{p}+\boldsymbol{i p} \mathrm{SC}$ decorations}

Apart from the Kitaev chain and complex fermion layers, there is an additional layer of $2 \mathrm{D} p+i p$ chiral SC decorations specified by $n_{1} \in H^{1}\left(G_{b}, \mathbb{Z}_{T}\right)$ for 3D FSPT states. The decoration of this layer is possible only when $G_{b}$ is not unitary. Since there are no fixed-point wave function constructions of chiral states on a discrete lattice [80], we discuss it after all the other layers in this section. However, we can put a $2 \mathrm{D}$ continuum (infinite number of degrees of freedom) free-fermion $p+i p \mathrm{SC}$ state on the decoration plane with the bulk mass approaching positive infinity. As shown below, the obstruction function for the $p+i p$ chiral superconductor decoration is $\omega_{2} \smile n_{1}+s_{1} \smile n_{1} \smile n_{1}$. For the $3 \mathrm{D}$ topological superconductor with time-reversal symmetry $T^{2}=-1$, the obstruction function equals zero identically.
So this $p+i p$ layer does not twist the obstruction functions of other higher layers. In this way, we can fully classify $3 \mathrm{D}$ $T^{2}=-1$ topological superconductors.

\section{Consistency condition}

We use $n_{1}\left(g_{i}, g_{j}\right) \in \mathbb{Z}$ to indicate the number of decorated $p+i p$ chiral superconductor layers on the plane dual to link $\langle i j\rangle$ (see Fig. 15). If $n_{1}\left(g_{i},{ }_{j}\right)<0$, we decorate inverse $p+i p$, i.e., $p-i p$, chiral superconductors. So the number of chiral Majorana modes on the boundary of the plane is $\left|n_{1}\left(g_{i}, g_{j}\right)\right|$. The direction of the chiral Majorana modes (see red arc arrows in Fig. 15) forms a right-hand (left-hand) rule with respect to the oriented link $\langle i j\rangle$ if $n_{1}\left(g_{i},{ }_{j}\right)>0\left[n_{1}\left(g_{i},{ }_{j}\right)<0\right]$. For a triangle $\langle i j k\rangle$, there are three $p+i p$ superconductor planes intersecting at the link dual to the triangle (see the red link in Fig. 15). Since we are constructing a gapped state, there should be no chiral Majorana mode along this link. So we have the gappable condition:

$\left(d n_{1}\right)\left(g_{0}, g_{1}, g_{2}\right)={ }^{g_{0}} n_{1}\left(g_{1}, g_{2}\right)-n_{1}\left(g_{0}, g_{2}\right)+n_{1}\left(g_{0}, g_{1}\right)=0$,

which merely states that the number of left-moving and right-moving chiral Majorana modes along the (red) link dual to triangle $\langle 012\rangle$ equal each other.

We explain the physical meaning of the $g_{0}$ symmetry action in the first term of Eq. (250). To be more precise, the first step of our $p+i p$ superconductor decoration is to put $n_{1}\left(g_{0}^{-1} g_{1}\right)$ (we use inhomogeneous notation for 1-cochain) layers of $p+i p$ superconductors to the plane dual to the standard link $\left\langle e, g_{0}^{-1} g_{1}\right\rangle$, which has an identity group

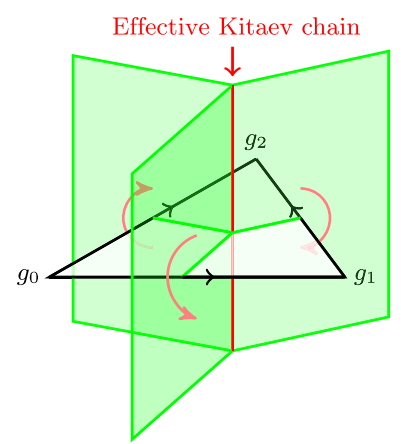

FIG. 15. Decorations of $p+i p$ chiral superconductors for a triangle $\langle 012\rangle$ [see Fig. 8(b) for a tetrahedron]. The 2D chiral superconductors are decorated on the (green) planes dual to the three links $\langle 01\rangle,\langle 12\rangle$, and $\langle 02\rangle$. The direction of the chiral Majorana mode along the boundary of the (green) plane dual to link $\langle i j\rangle$ is indicated by a (red) arc arrow (right-hand rule with respect to the link direction) if $n_{1}>0$. So there are three different kinds of chiral Majorana modes along the (red) link dual to the triangle $\langle 012\rangle$. After gapping out these chiral modes symmetrically, there may be an effective Kitaev chain along the (red) link. 
element label for the first vertex. By applying a $U\left(g_{0}\right)$ symmetry action on this state, the group element label of the link becomes $\left\langle g_{0}, g_{1}\right\rangle$. At the same time, if $g_{0}$ is time reversal, the decorated $p+i p$ superconductors should become $p-i p$ under the $U\left(g_{0}\right)$ action; i.e., $n_{1}\left(g_{0}^{-1} g_{1}\right)$ becomes $-n_{1}\left(g_{0}^{-1} g_{1}\right)$. After using the homogeneous notation and counting the directions of the left and right directions of the chiral Majorana modes, we obtain the cocycle equation of $n_{1}$ with mathematically correct symmetry action in Eq. (250).

If $g \in G_{b}$ is unitary, then one can show $n_{1}\left(g^{k}\right)=k n_{1}(g)$ for all $k \in \mathbb{Z}$ from Eq. (250). Since we consider only finite $G_{b}$, we must have $n_{1}(g)=0$ for all unitary $g \in G_{b}$. So $H^{1}\left(G_{b}, \mathbb{Z}_{T}\right)$ is trivial if $G_{b}$ is a finite unitary group, and there is no $p+i p$ chiral superconductor decoration layer. For symmetry group $G_{b}$ with antiunitary elements, one can show

$$
n_{1}(g)= \begin{cases}0, & g \text { is unitary, } \\ 1, & g \text { is antiunitary }\end{cases}
$$

by adding some coboundaries. So we have $H^{1}\left(G_{b}, \mathbb{Z}_{T}\right)=$ $\mathbb{Z}_{2}$ for $G_{b}$ with antiunitary elements.

\section{Symmetry transformations}

The chiral Majorana modes along the boundary of the plane dual to link $\langle i j\rangle$ are denoted by $\psi_{i j, R ; \alpha}^{g_{i}}$ or $\psi_{i j, L ; \alpha}^{g_{i}}$ $\left(\alpha=1,2, \ldots,\left|n_{1}(i j)\right|\right)$ for $n_{1}(i j)>0$ or $n_{1}(i j)<0$, respectively. As discussed in Sec. VIA, the symmetry transformation rules for the chiral Majorana modes are given by Eqs. (171) and (172). The $\omega_{2}$ part is the usual projective representation factor for $G_{b}$ (such that it is a linear representation of $G_{f}$ ). The $s_{1}$ part is more subtle, which needs some explanation.

To be more specific, the time-reversal symmetry acts on the chiral Majorana modes as

$$
\begin{gathered}
U(T) \psi_{i j, R ; \alpha}^{g_{i}} U(T)^{\dagger}=(-1)^{\omega_{2}\left(T, g_{i}\right)} \psi_{i j, L ; \alpha}^{T g_{i}}, \\
U(T) \psi_{i j, L ; \alpha}^{g_{i}} U(T)^{\dagger}=-(-1)^{\omega_{2}\left(T, g_{i}\right)} \psi_{i j, R ; \alpha}^{T g_{i}} .
\end{gathered}
$$

Basically, it changes $\psi_{R}$ to $\psi_{L}$ and $\psi_{L}$ to $-\psi_{R}$ (forgetting the $\omega_{2}$ factor). So the left-moving and right-moving chiral Majorana modes form a time-reversal Kramers doublet with $T^{2}=-1$. The reason to choose this symmetry transformation convention is as follows. If we fold the three (green) planes in Fig. 15 into one plane, we should obtain a symmetric state on the pure 2D plane. The system consists of several copies of $p+i p$ and $p-i p$ chiral superconductors. However, it is known that we have only nontrivial 2D topological superconductors for time-reversal symmetry $T^{2}=-1$ [76]. The $T^{2}=1$ case corresponds to the ASPT state, which can exist only on the boundary of a 3D bulk [66] (see also the FSPT classification examples in Appendix E2). Therefore, we choose the chiral Majorana modes to form Kramers doublet under time-reversal symmetry.

\section{Symmetric mass terms}

To obtain a symmetric gapped state, we should add symmetric mass terms to the three kinds of chiral Majorana modes along the link dual to each triangle $\langle i j k\rangle$. The strategy is again first considering the standard mass term for the standard triangle with first vertex label $e \in G_{b}$. The standard mass term has the following form:

$$
i m \psi_{\text {out }}^{e} \psi_{\text {in }}^{g_{0}^{-1} g_{i}} \text { or } i m \psi_{\text {out }}^{g_{0}^{-1} g_{i}} \psi_{\text {in }}^{e},
$$

where at least one of the two chiral Majorana modes is labeled by the identity element $e$ of $G_{b}$. The subscripts "out" and "in" indicate the actual direction of the chiral Majorana modes along the (red) link dual to the triangle, i.e., going outside or inside the triangle (using the righthand rule). This direction is not locked with the sign of $n_{1}$, for the three links of the triangle have different right-hand rule directions (see the red arc arrows in Fig. 15). We always put an outgoing mode in front of an ingoing mode, because we have to set a rule to know whether there is an effective Kitaev chain compared to the "trivial" gapped edge by checking simply the sign of the mass.

Other nonstandard mass terms are obtained from the standard one by a symmetry action. There is an additional minus sign for the mass from the symmetry action. The negative mass induces an effective Kitaev chain along the gapped edge (see Fig. 15). In the following, we consider separately the symmetric mass terms for nontrivial $\omega_{2}$ and $s_{1}$.

(a) Mass term signs from $\omega_{2}$.-We first consider the standard mass terms for the standard triangle $\langle 012\rangle$ with group element labels $e, g_{0}^{-1} g_{1}$, and $g_{0}^{-1} g_{2}$ for three vertices. According to Eq. (250), the number of left-moving and right-moving modes along the (red) link dual to the triangle should be the same. For simplicity, we assume all of $n_{1}(01)=n_{1}\left(e, g_{0}^{-1} g_{1}\right), \quad n_{1}(12)=n_{1}\left(g_{0}^{-1} g_{1}, g_{0}^{-1} g_{2}\right), \quad$ and $n_{1}(02)=n_{1}\left(e, g_{0}^{-1} g_{2}\right)$ are positive integers and satisfy $n_{1}(02)=n_{1}(01)+n_{1}(12)$. Then, we need two types of standard mass terms to gap out these modes (assume $m>0)$ :

$$
\begin{aligned}
H_{\mathrm{std}}^{\langle 012\rangle}= & \sum_{\alpha=1}^{n_{1}(01)} i m \int d x \psi_{01, R ; \alpha}^{e}(x) \psi_{02, R ; \alpha}^{e}(x) \\
& +\sum_{\alpha=1}^{n_{1}(12)} i m \int d x \psi_{12, R ; \alpha}^{g_{0}^{-1} g_{1}}(x) \psi_{02, R ; \alpha}^{e}(x) .
\end{aligned}
$$

We note that the chiral modes $\psi_{01, R ; \alpha}^{e}$ and $\psi_{12, R ; \alpha}^{g_{0}^{-1} g_{1}}$ are going outside the triangle, while $\psi_{02, R ; \alpha}^{e}$ is inside (see the red arc 
arrows in Fig. 15). So it satisfies the standard mass term rule Eq. (254).

For the nonstandard triangle with vertex label $g_{0}, g_{1}$, and $g_{2}$, we should use a symmetry action to obtain the mass terms:

$$
\begin{aligned}
H_{\text {mass }}^{\langle 012\rangle}= & U\left(g_{0}\right) H_{\text {std }}^{\langle 012\rangle} U\left(g_{0}\right)^{\dagger} \\
= & \sum_{\alpha=1}^{n_{1}(01)} i m \int d x \psi_{01, R ; \alpha}^{g_{0}}(x) \psi_{02, R ; \alpha}^{g_{0}}(x) \\
& +\sum_{\alpha=1}^{n_{1}(12)}(-1)^{\omega_{2}\left(g_{0}, g_{0}^{-1} g_{1}\right)} i m \int d x \psi_{12, R ; \alpha}^{g_{1}}(x) \psi_{02, R ; \alpha}^{g_{0}}(x),
\end{aligned}
$$

where the sign $(-1)^{\omega_{2}\left(g_{0}, g_{0}^{-1} g_{1}\right)}$ of the second type of mass term comes from the symmetry transformation rule for $\psi_{12, R ; \alpha}^{g_{0}^{-1} g_{1}}$. The total number of effective Kitaev chain going through the triangle $\langle 012\rangle$ is then

$$
\omega_{2}\left(g_{0}, g_{0}^{-1} g_{1}\right) n_{1}\left(g_{1}^{-1} g_{2}\right),
$$

which is just the number of negative mass terms in Eq. (256).

If the three $n_{1}$ are not all positive, the mass terms are different from Eq. (256). In general, there are three types of mass terms which gap out different pairs of chiral Majorana modes. The standard forms of these three types are

$$
\begin{aligned}
& H_{\mathrm{std}}^{01,02}=\sum_{\alpha=1}^{N-\left|n_{1}(12)\right|} i m \int d x \psi_{01 ; \alpha}^{e}(x) \psi_{02 ; \alpha}^{e}(x), \\
& H_{\mathrm{std}}^{12,02}=\sum_{\alpha=1}^{N-\left|n_{1}(01)\right|} i m \int d x \psi_{12 ; \alpha}^{g_{0}^{-1} g_{1}}(x) \psi_{02 ; \alpha}^{e}(x), \\
& H_{\mathrm{std}}^{01,12}=\sum_{\alpha=1}^{N-\left|n_{1}(02)\right|} i m \int d x \psi_{01 ; \alpha}^{e}(x) \psi_{02 ; \alpha}^{g_{0}^{-1} g_{1}}(x),
\end{aligned}
$$

where we define $N=\max \left[\left|n_{1}(01)\right|,\left|n_{1}(12)\right|,\left|n_{1}(02)\right|\right]$. Note that (at most) only two types of mass terms appear for a given $n_{1}$ configuration. We should choose the mass terms which involve the chiral modes corresponding to the biggest $\left|n_{1}\right|$. For example, the two types of mass terms in Eq. (255) both involve $\psi_{02}$, because $n_{1}(02)>0$ is the biggest and $N-\left|n_{1}(02)\right|=0$ for the summation in Eq. (260). The order of the two Majorana modes in a mass term should follow the rule Eq. (254) that the outgoing mode appears in the front. It depends on the configuration of $n_{1}$. The above three equations are only one example. (We also omit the $R / L$ label of the chiral modes, for the sign of $n_{1}$ is indefinite.)
Independent of the signs of $n_{1}$, the effective Kitaev chain number is always Eq. (257). The minus signs of the masses all come from the symmetry sign $(-1)^{\omega_{2}\left(g_{0}, g_{0}^{-1} g_{1}\right)}$ of $\psi_{12, R / L ; \alpha}^{g_{0}^{-1} g_{1}}$. Independent of the signs of $n_{1}$, there are always $\left|n_{1}(12)\right|$ mass terms associated with this chiral modes [see Eqs. (259) and (260)]. Under a $U\left(g_{0}\right)$ action from the standard mass term to the actual mass term, all the terms in Eqs. (259) and (260) have negative masses. So the $(\bmod 2)$ number of effective Kitaev chains is always Eq. (257).

(b) Mass term signs from $s_{1}$. - There are additional signs for the mass terms related to time-reversal symmetry. In general, our standard mass term has the form (we omit the group element labels)

$$
i m \int d x \psi_{\mathrm{out}, s}(x) \psi_{\mathrm{in}, s^{\prime}}^{\prime}(x)
$$

where the outgoing mode (going up in Fig. 15) is in front of the ingoing mode (going down in Fig. 15). The labels $s$ and $s^{\prime}$ denote $R$ or $L$, depending on $n_{1}>0$ or $n_{1}<0$. Note that the outgoing or ingoing is not locked with $R / L$ (see the red arc arrows in Fig. 15).

Under a unitary symmetry action $U\left(g_{0}\right)$, the mass term is transformed to

$$
\begin{aligned}
& i m \int d x \psi_{\mathrm{out}, s}(x) \psi_{\mathrm{in}, s^{\prime}}^{\prime}(x) \\
& \underset{U\left(g_{0}\right)}{\stackrel{\text { unitary }}{\longrightarrow}}(-1)^{\omega_{2}} i m \int d x \psi_{\mathrm{out}, s}(x) \psi_{\mathrm{in}, s^{\prime}}^{\prime}(x),
\end{aligned}
$$

where the sign $(-1)^{\omega_{2}}$ is exactly the transformation sign of $\psi^{g_{0}^{-1} g_{1}}$ discussed previously. So we can obtain the effective Kitaev chain number Eq. (257) in a simple way.

On the other hand, if the symmetry $U\left(g_{0}\right)$ is antiunitary, it reverses the directions of all the chiral modes. The action on the standard mass term Eq. (261) is

$$
\begin{aligned}
& i m \int d x \psi_{\text {out }, s}(x) \psi_{\mathrm{in}, s^{\prime}}^{\prime}(x) \\
& \underset{U\left(g_{0}\right)}{\stackrel{\text { antiunitary }}{\longrightarrow}}(-1)^{\omega_{2}}(-1)^{1+1+\left(1-\delta_{s, s^{\prime}}\right)} i m \int d x \psi_{\mathrm{out},-s^{\prime}}^{\prime}(x) \psi_{\mathrm{in},-s}(x) .
\end{aligned}
$$

Apart from the $\omega_{2}$ term, there are three additional signs. One minus sign comes from the antiunitary action on the imaginary unit $i$. The second is the sign of switching two chiral modes $\psi$ and $\psi^{\prime}$, because time reversal changes the outgoing modes to ingoing modes, and vice versa. According to the rule Eq. (254), we should change their orders. The third sign $(-1)^{1-\delta_{s, s^{\prime}}}$ appears only when $s$ and $s^{\prime}$ are different; i.e., the two modes are of different $R / L$ types. This sign is a consequence of the symmetry transformation rules Eqs. (252) and (253): $R \rightarrow L$ and $L \rightarrow-R$. The only 
mass term between $R$ - and $L$-type modes is Eq. (260) for $\psi_{01, R / L}$ and $\psi_{01, L / R}$. Using the "canonical" 1-cocycle Eq. (251), we conclude that the only mass term with a (time-reversal-related) minus sign is -im $\psi_{12}^{g_{1}} \psi_{01}^{g_{0}}$ with antiunitary $g_{0}$. This case corresponds to $s_{1}\left(g_{0}\right)=1-s_{1}\left(g_{1}\right)=$ $s_{1}\left(g_{2}\right)=1$ and $n_{1}(12)=-n_{1}(01)=1-n_{1}(02)=1$. We can summarize this (time-reversal-related) sign for the mass term by $(-1)^{s_{1}\left(g_{0}\right) n_{1}(\overline{0} 1) n_{1}(\overline{1} 2)}$. So the $(\bmod 2)$ number of effective Kitaev chain going through the triangle related to time-reversal symmetry is

$$
s_{1}\left(g_{0}\right) n_{1}\left(g_{0}^{-1} g_{1}\right) n_{1}\left(g_{1}^{-1} g_{2}\right) .
$$

In summary, we can use symmetric mass terms to gap out all the chiral Majorana modes along the (red) link dual to the triangle $\langle 012\rangle$. There are effective Kitaev chains left along the link, with (mod 2) number

$$
\begin{aligned}
& \left(\omega_{2} \smile n_{1}+s_{1} \smile n_{1} \smile n_{1}\right)\left(g_{0}, g_{0}^{-1} g_{1}, g_{1}^{-1} g_{2}\right) \\
& \quad=\omega_{2}\left(g_{0}, g_{0}^{-1} g_{1}\right) n_{1}\left(g_{1}^{-1} g_{2}\right)+s_{1}\left(g_{0}\right) n_{1}\left(g_{0}^{-1} g_{1}\right) n_{1}\left(g_{1}^{-1} g_{2}\right),
\end{aligned}
$$

which is a combination of Eqs. (257) and (264).

\section{Obstruction function}

If we consider a tetrahedron $\langle 0123\rangle$ of the 3D triangulation lattice of the spatial manifold, there are four triangles on the boundary and four (red) links with chiral Majorana modes [see Fig. 8(b)]. We should add mass terms $H_{\text {mass }}^{\langle 123\rangle}$, $H_{\text {mass }}^{\langle 023\rangle}, H_{\text {mass }}^{\langle 013\rangle}$, and $H_{\text {mass }}^{\langle 012\rangle}$ for all the triangles, following the discussions above. The total number of effective Kitaev chains crossing the boundary of the tetrahedron can be calculated as the summation of four terms similar to Eq. (265). Using the cocycle equations for $\omega_{2}, s_{1}$, and $n_{1}$, we have $d\left(\omega_{2} \smile n_{1}+s_{1} \smile n_{1} \smile n_{1}\right)\left(g_{0}, g_{0}^{-1} g_{1}, g_{1}^{-1} g_{2}\right.$, $\left.g_{2}^{-1} g_{3}\right)=0$. So the total $(\bmod 2)$ number of effective Kitaev chains for the tetrahedron is

$$
\left(\omega_{2} \smile n_{1}+s_{1} \smile n_{1} \smile n_{1}\right)\left(g_{0}^{-1} g_{1}, g_{1}^{-1} g_{2}, g_{2}^{-1} g_{3}\right) .
$$

To make sure that there are no dangling Majorana fermion inside any tetrahedron of the lattice, the number of effective Kitaev chains should equal the number of decorated Kitaev chains specified by $n_{2}$. So we have the consistency equation

$$
d n_{2}=\omega_{2} \smile n_{1}+s_{1} \smile n_{1} \smile n_{1} .
$$

If the right-hand side of the above equation is not a $\mathbb{Z}_{2}$-valued 3-coboundary, there is no solution for $n_{2}$ on the left-hand side. We note that the above equation is the same as the 1D consistency equation [Eq. (119)], although the physical meanings are totally different.

\section{G. Classification of 3D FSPT phases}

The general classification of 3D FSPT phases is as follows. We first calculate the cohomology groups $H^{1}\left(G_{b}, \mathbb{Z}_{T}\right)$, $H^{2}\left(G_{b}, \mathbb{Z}_{2}\right), H^{3}\left(G_{b}, \mathbb{Z}_{2}\right)$, and $H^{4}\left(G_{b}, U(1)_{T}\right)$. For each $n_{1} \in H^{1}\left(G_{b}, \mathbb{Z}_{T}\right)$, we solve the twisted cocycle equation Eq. (18) for $n_{2}$. For each solution $n_{2}$, we solve the twisted cocycle equation [Eq. (18)] for $n_{3}$. And for each solution $n_{3}$, we solve the twisted cocycle equation [Eq. (18)] for $\nu_{4}$. If $n_{2}$, $n_{3}$, and $\nu_{4}$ are in the trivialization subgroup $\Gamma^{2}, \Gamma^{3}$, and $\Gamma^{4}$ in Eq. (19), then they are trivialized by boundary ASPT states. (Similar to the 1D and 2D case, we can define $\Gamma^{4}$ to describe those 3D BSPT states trivialized by fermions.) So the obstruction-free and trivialization-free $\left(n_{1}, n_{2}, n_{3}, \nu_{4}\right)$ fully classify the 3D FSPT phases.

We can also use the 3D FSLU transformations to construct the commuting-projector parent Hamiltonians. The procedure is again tedious but straightforward. Each term of the Hamiltonian is a sequence of 3D fermionic $F$ moves that changes the group element label of a vertex from $g_{*}$ to $g_{*}^{\prime}$. Different terms commute with each other, for the 3D FSPT wave function is at the fixed point.

\section{CONCLUSIONS AND DISCUSSIONS}

In this paper, we construct gapped fermionic state with symmetry $G_{f}$ by decorating fermionic degrees of freedom. In $d$ spatial dimensions, they are constructed using several layers of data $\left(\ldots, n_{d-1}, n_{d}, \nu_{d+1}\right)$, which is an element in $\ldots \times C^{d-1}\left(G_{b}, \mathbb{Z}_{2}\right) \times C^{d}\left(G_{b}, \mathbb{Z}_{2}\right) \times C^{d+1}\left[G_{b}, U(1)_{T}\right]$ (see Table I). There are several consistency conditions for them. Basically, the coboundary of one layer data should equal a functional of the data of lower layers (see Table II). We can summarize them as a system of twisted cocycle equations:

$$
d\left(\ldots, n_{d-1}, n_{d}, \nu_{d+1}\right)=\left(\ldots, \mathcal{O}_{d}, \mathcal{O}_{d+1}, \mathcal{O}_{d+2}\right) .
$$

Note that the obstruction function $\mathcal{O}_{d+2}$ is a $U(1)_{T}$-valued $(d+2)$-cocycle. And all other obstruction functions $\mathcal{O}_{i}$ $(i \leq d+1)$ are in $H^{i}\left(G_{b}, \mathbb{Z}_{2}\right)$ with $\mathbb{Z}_{2}$ coefficients. The data $\left(\ldots, n_{d-1}, n_{d}, \nu_{d+1}\right)$ correspond to a valid FSPT state if and only if all the obstruction functions are coboundaries. Otherwise, there are no solutions for data of the next layer.

There are two related questions about the constructed FSPT states. The first is whether the states with different $\left(\ldots, n_{d-1}, n_{d}, \nu_{d+1}\right)$ data represent distinct FSPT phases. If we have a path of FSLU transformations to connect them, they are, in fact, in the same phase. So the FSPT classification data should quotient these cases. The second question is what happens to the state that is obstructed by some nontrivial cocycle $\mathcal{O}_{i}$. There are some physical inconsistencies for these states, because all the obstruction functions have physical meanings such as fermion parity conservation (see the last column in Table II). But is it possible to construct such a state on the boundary of a one-higher-dimensional state? If it is 
possible, we need to understand the physical properties of the bulk, such as whether it is long-range entangled or short-range entangled.

The answers of the above two questions are related to the concept of anomalous SPT states [66]. If one of the obstruction functions on the right-hand side of Eq. (268) is not a coboundary, the state is obstructed, for it violates some physical consistency constraints (see the last column in Table II). However, this state can exist as an ASPT state on the boundary of an FSPT state in $(d+1)$ spatial dimension. The ASPT states in $(d-1)$ spatial dimensions induces new kinds of coboundaries for the classification data of FSPT phases in $d$ dimensions $[52,53,66]$. Formally, we can write the new coboundaries as

$$
\begin{aligned}
\left(\ldots, n_{d-1}, n_{d}, \nu_{d+1}\right) & \sim\left(\ldots, n_{d-1}, n_{d}, \nu_{d+1}\right)+d\left(\ldots, n_{d-2}, n_{d-1}, \nu_{d}\right) \\
& =\left(\ldots, n_{d-1}, n_{d}, \nu_{d+1}\right)+\left(\ldots, \mathcal{O}_{d-1}, \mathcal{O}_{d}, \mathcal{O}_{d+1}\right)
\end{aligned}
$$

In the first line of the above equation, we identify the FSPT state with $\left(\ldots, n_{d-1}, n_{d}, \nu_{d+1}\right)$ with another state by stacking the coboundary of one lower dimension state with data $\left(\ldots, n_{d-2}, n_{d-1}, \nu_{d}\right)$. The coboundary of the latter data, by Eq. (268), are exactly the obstruction functions for the FSPT in one lower dimensions. Therefore, the lower-dimensional FSPT obstruction functions will trivialize the higher-dimensional FSPT data.

Mathematically, the obstruction functions $\mathcal{O}_{i}$ for FSPT states in $(d-1)$ spatial dimensions form a subgroup of the cohomology group $H^{i}\left(G_{b}, \mathbb{Z}_{2}\right)$ (or $H^{i}\left[G_{b}, U(1)_{T}\right]$ ):

$$
\Gamma^{i}=\left\{\mathcal{O}_{i}\left[n_{i-2}\right] \mid n_{i-2} \in C^{i-2}\left(G_{b}, \mathbb{Z}_{2}\right) \text { is a classification data for FSPT in }(d-1) \text { dimensions }\right\} .
$$

If $\mathcal{O}_{i}\left[n_{i-2}\right]$ is a nontrivial cocycle in $\Gamma^{i}$, the $(d-1)$ dimensional state with classification data $n_{i-2}$ is obstructed. On the other hand, if the classification data $n_{i}$ (or $\nu_{i}$ ) for $d$ dimensional FSPT state belong to the subgroup $\Gamma^{i}$, it is trivialized because of the boundary ASPT state. Therefore, the distinct classification data for a $d$-dimensional FSPT state is, in fact, $n_{i} \in C^{i}\left(G_{b}, \cdot\right) / B^{i}\left(G_{b}, \cdot\right) / \Gamma^{i}$ [the coefficient is $\mathbb{Z}_{2}$ or $\left.U(1)_{T}\right]$.

For each solution of Eq. (268), we can use the classification data to construct an FSPT state by decorating several layers of fermionic degrees of freedom to the BSPT state. These states belong to different FSPT phases if the data are different in $C^{i}\left(G_{b}, \cdot\right) / B^{i}\left(G_{b}, \cdot\right) / \Gamma^{i}$. We can also use the FSPT moves to construct commuting-projector parent Hamiltonians. The Hamiltonian consists of a local operator which corresponds to a sequence of $F$ moves that changes one vertex label from $g_{*}$ to $g_{*}^{\prime}$. Different terms commute with each other, because the $F$ moves satisfy the coherence conditions.

We conjecture that, in principle, our classification scheme for FSPT phases can also be applied to point and space group symmetry, so long as the crystalline principle and spin statistics relations [82] are carefully considered. For example, the mirror symmetry with $\sigma^{2}=1$ $\left(\mathbb{Z}_{2}^{f} \times \mathbb{Z}_{2}^{P}\right.$ ) should be regarded as a time-reversal symmetry with $T^{2}=P_{f}\left(\mathbb{Z}_{4}^{T f}=\mathbb{Z}_{2}^{f} \times_{\omega_{2}} \mathbb{Z}_{2}^{T}\right)$, while the mirror symmetry with $\sigma^{2}=P_{f} \quad\left(\mathbb{Z}_{4}^{T P f}=\mathbb{Z}_{2}^{f} \times_{\omega_{2}} \mathbb{Z}_{2}^{P}\right)$ should be regarded as a time-reversal symmetry with $T^{2}=1$ $\left(\mathbb{Z}_{2}^{f} \times \mathbb{Z}_{2}^{T}\right.$ ) [83]. The full details for the classification and construction of point and space group protected FSPT phases will be presented in our future work. Moreover, we also believe that our construction and classification scheme can be applied for continuum Lie group symmetry by using the Borel cohomology. However, it is very difficult to compute the obstruction functions for the general Lie group, and we will develop special tools to handle this problem in the future. Finally, how to generalize our framework into FSPT phases protected by supersymmetry will be an extremely interesting future direction.

\section{ACKNOWLEDGMENTS}

We are grateful to X.-G. Wen for very enlightening discussions. We also thank Meng Cheng, Yang Qi and Chenjie Wang for stimulation discussions on ASPT phases. This work is supported by Direct Grant No. 4053300 from The Chinese University of Hong Kong and funding from Hong Kong's Research Grants Council (GRF No. 14306918, ANR/RGC Joint Research Scheme No. A-CUHK402/18).

\section{APPENDIX A: FIXED-POINT WAVE FUNCTION AND CLASSIFICATION OF FSPT STATES IN OD}

In this appendix, we discuss the FSPT states in zero spatial dimensions, which are classified by the onedimensional representations of $G_{f}$, i.e., $H^{1}\left[G_{f}, U(1)_{T}\right]$. As shown below, we can choose equivalently the classification data to be $n_{0} \in H^{0}\left(G_{b}, \mathbb{Z}_{2}\right)$ and $\nu_{1} \in C^{1}\left[G_{b}, U(1)_{T}\right] /$ $B^{1}\left[G_{b}, U(1)_{T}\right]$ with some consistency equations. Although the $0 \mathrm{D}$ case is rather degenerate, it shows the layer structure of the FSPT classifications, which is also true but more complicated in higher dimensions. 


\section{Classification}

It is known that the OD BSPT states with symmetry group $G_{b}$ are classified by the one-dimensional linear representations of $G_{b}$, i.e., $H^{1}\left[G_{b}, U(1)_{T}\right]$ [4]. This classification is because the SPT state should be both symmetric and nondegenerate. In zero spatial dimensions, there is essentially no difference between bosonic and fermionic systems, except that there is an additional $\mathbb{Z}_{2}^{f}$ symmetry for the fermionic system. We can treat a fermionic system with total fermionic symmetry group $G_{f}=\mathbb{Z}_{2}^{f} \times_{\omega_{2}} G_{b}$ as a bosonic system with total bosonic symmetry group $G_{f}$. Therefore, we have the following conclusion:

(i) OD FSPT phases with symmetry group $G_{f}=\mathbb{Z}_{2}^{f} \times_{\omega_{2}}$ $G_{b}$ are classified by the one-dimensional irreducible representations of $G_{f}$, i.e., $H^{1}\left[G_{f}, U(1)_{T}\right]$.

Equivalently, we can unpack the above result and show that

(i) OD FSPT phases with symmetry group $G_{f}=\mathbb{Z}_{2}^{f} \times_{\omega_{2}}$ $G_{b}$ are classified by a 0 -cocycle $n_{0}$ and a 1 -cochain $\nu_{1}$, with some symmetry conditions and consistency equations.

The second version of classification is more physical. The first data $n_{0} \in H^{0}\left(G_{b}, \mathbb{Z}_{2}\right)$ is related to the fermion parity of the state: $P_{f}=(-1)^{n_{0}}$. The second data $\nu_{1} \in$ $C^{1}\left[G_{b}, U(1)_{T}\right] / B^{1}\left[G_{b}, U(1)_{T}\right]$ is the usual 0D BSPT classification.

To get a sense of the classification, we first consider the simpler case of $G_{f}=\mathbb{Z}_{2}^{f} \times G_{b}$. Using the Künneth formula, we can split the one-dimensional representation of $G_{f}=\mathbb{Z}_{2}^{f} \times G_{b}$ into two parts: $H^{1}\left[G_{f}, U(1)_{T}\right]=$ $H^{1}\left[\mathbb{Z}_{2}^{f}, U(1)_{T}\right] \times H^{1}\left[G_{b}, U(1)_{T}\right]=\mathbb{Z}_{2} \times H^{1}\left[G_{b}, U(1)_{T}\right]$. The first $\mathbb{Z}_{2}$ part corresponds to the one-dimensional representation of $\mathbb{Z}_{2}^{f}$, indicating the bosonic or fermionic nature of the state. We can use the value of fermion parity $P_{f}=(-1)^{n_{0}}\left(n_{0} \in \mathbb{Z}_{2}=\{0,1\}\right)$ to represent this $\mathbb{Z}_{2}$ classification. The second part is the same as the bosonic counterpart, which is the one-dimensional irreducible representation of $G_{b}$.

Now let us consider the generic case $G_{f}=\mathbb{Z}_{2}^{f} \times_{\omega_{2}} G_{b}$ obtained by Eq. (1). In general, for a given onedimensional representation $\tilde{U}$ of $G_{f}$, we can always separate $\tilde{U}\left(P_{f}^{n} g\right)$ [with $g \in G_{b}$ and $P_{f}^{n} g=\left(P_{f}^{n}, g\right) \in G_{f}$ ] into three parts:

$$
\tilde{U}\left(P_{f}^{n} g\right)=P_{f}^{n} \nu_{1}(g) K^{s_{1}(g)},
$$

where $\nu_{1}(g)$ is a $U(1)$ phase factor and $K$ is the complex conjugation operator depending on whether $g$ contains time reversal or not. Using the multiplication rule [Eq. (2)] of $G_{f}$, the representation condition $\tilde{U}\left(P_{f}^{n} g\right) \tilde{U}\left(P_{f}^{m} h\right)=$ $\tilde{U}\left(P_{f}^{n} g \cdot P_{f}^{m} h\right)$ becomes

$$
\nu_{1}(g) \nu_{1}(h)^{1-2 s_{1}(g)}=P_{f}^{\omega_{2}(g, h)} \nu_{1}(g h) .
$$

When acting on a state with fixed fermion parity $(-1)^{n_{0}}$ [we can again think of $n_{0} \in H^{0}\left(G_{b}, \mathbb{Z}_{2}\right)=\mathbb{Z}_{2}$ as a 0 -cocycle], the above equation can be summarized as

$\left(d \nu_{1}\right)(g, h):=\frac{\nu_{1}(h)^{1-2 s_{1}(g)} \nu_{1}(g)}{\nu_{1}(g h)}=(-1)^{\left(\omega_{2} \smile n_{0}\right)(g, h)}$,

which means that the cocycle equation of $\nu_{1}$ is twisted by $\omega_{2} \smile n_{0}$. If we define the homogeneous $\nu_{1}$ by the inhomogeneous one as $\nu_{1}(g, g a)={ }^{g} \nu_{1}(a)=$ $\nu_{1}(a) \cdot(-1)^{\left(\omega_{2} \smile n_{0}\right)(g, a)}$ (we omit the superscript $g$ of the inhomogeneous ${ }^{g} \nu_{1}$ if $g=e$ is the identity element of $G_{b}$ ), we obtain the symmetry conditions and consistency equations for $n_{0}$ and $\nu_{1}$. It is easy to see that, under the condition $\omega_{2}=0$, the classification is reduced to the previous discussed case $G_{f}=\mathbb{Z}_{2}^{f} \times G_{b}$ where $n_{0}$ and $\nu_{1}$ are decoupled cocycles.

\section{Fixed-point wave functions}

The above discussion on the OD FSPT state is from the perspective of symmetry representation $\tilde{U}$. We can also construct fixed-point wave functions.

For $n_{0}=0$, the wave function is fermion parity even. Using the basis state $|\sigma\rangle$ with symmetry transformation

$$
\tilde{U}\left(P_{f}^{n} g\right)|\sigma\rangle=|g \sigma\rangle,
$$

we can construct the fixed-point wave function as

$$
|\Psi\rangle=\sum_{\sigma \in G_{b}} \nu_{1}(\sigma)^{-1}|\sigma\rangle
$$

It is easy to check that the wave function supports a onedimensional representation of $G_{f}$ :

$$
\tilde{U}\left(P_{f}^{n} g\right)|\Psi\rangle=\nu_{1}(g)|\Psi\rangle .
$$

For $n_{0}=1$, the wave function is a fermionic state. The basis state is created by a fermion creation operator as $\left(\sigma \in G_{b}\right)$

$$
|\sigma\rangle=c_{\sigma}^{\dagger}|0\rangle .
$$

The symmetry transformation of the basis state under $G_{f}$ is

$$
\tilde{U}\left(P_{f}^{n} g\right)|\sigma\rangle=(-1)^{n}|g \sigma\rangle .
$$

The fixed-point wave function is also a superposition of all basis states:

$$
|\Psi\rangle=\sum_{\sigma \in G_{b}} \nu_{1}(\sigma)^{-1}|\sigma\rangle
$$


with odd fermion parity. One can check the one-dimensional representation of $G_{f}$ on this fixed-point wave function:

$$
\tilde{U}\left(P_{f}^{n} g\right)|\Psi\rangle=(-1)^{n} \nu_{1}(g)|\Psi\rangle
$$

\section{Trivialization of some 1D BSPT states in fermionic system}

The obstruction function $(-1)^{\omega_{2} \smile n_{0}}$ on the right-hand side of Eq. (A3) comes from the projective representation Eq. (A2) of $G_{b}$ on $0 \mathrm{D}$ fermions. Although the $0 \mathrm{D}$ fermion state forms a projective representation of $G_{b}$, it is a onedimensional linear representation of $G_{f}$.

On the other hand, it is well known that the classification data $\nu_{2} \in H^{2}\left[G_{b}, U(1)\right]$ of 1D BSPT states also correspond to the projective representation of the bosonic symmetry group. The physical meaning is that the OD edge mode of this 1D BSPT open chain is a projective representation of $G_{b}$. Since the edge degeneracy cannot be lifted by symmetric terms, we conclude that the $1 \mathrm{D}$ bulk is a nontrivial BSPT.

Now let us consider the problem of embedding the 1D BSPT state with $\nu_{2}$ into a fermionic system. We want to know that whether this BSPT state is still nontrivial as an FSPT state. From the perspective of edge states, we can couple a OD fermionic state with projective representation $(-1)^{\omega_{2} \smile n_{0}}$ of $G_{b}$ to the boundary of this 1D BSPT state. Then, the total projective representation of the edge is $\nu_{2} \times(-1)^{\omega_{2} \smile n_{0}}$. If $\nu_{2} \in \Gamma^{2}=\left\{(-1)^{\omega_{2} \smile n_{0}} \mid n_{0} \in \mathbb{Z}_{2}\right\}$, the projective representation of the edge is, in fact, a linear representation by choosing appropriate $n_{0}$. So there is no nontrivial edge state in this fermionic system. We conclude that the BSPT states in $\Gamma^{2}$ are trivialized as a fermionic state.

The trivialization of some $d$-dimensional BSPT states in a fermionic system is also known even for the case of $G_{f}=\mathbb{Z}_{2}^{f} \times G_{b}$ [52]. In this case, the trivialization subgroup is $\Gamma^{d+1}=\left\{(-1)^{S q^{2}\left(n_{d-1}\right)} \mid n_{d-1} \in H^{d-1}\left(G_{b}, \mathbb{Z}_{2}\right)\right\} \subset$ $H^{d+1}\left[G_{b}, U(1)\right]$. There are more terms if we consider groups with nontrivial $\omega_{2}$ and $s_{1}$. But it is still exactly the obstruction function for FSPT states in one lower dimensions.

There is another way of obtaining the trivialization subgroup of the BSPT states from the perspective of FSLU. We can construct an explicit FSLU for an FSPT state [see Eqs. (46) and (47) in Ref. [53]]. The final result is that the $U(1)$ coefficient of the wave function is modified by an element of the trivialization subgroup $\Gamma^{d+1}$. Since the two FSPT states should be considered as in the same phase (they are connected by FSLU), we conclude that the BSPT states in $\Gamma^{d+1}$ are trivialized in fermionic system.

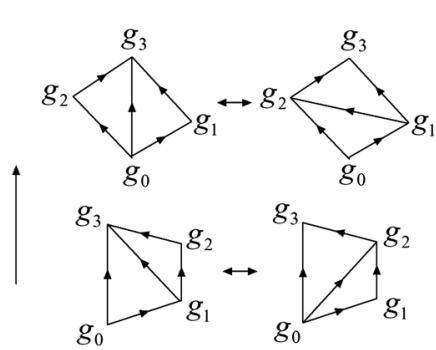

(a)
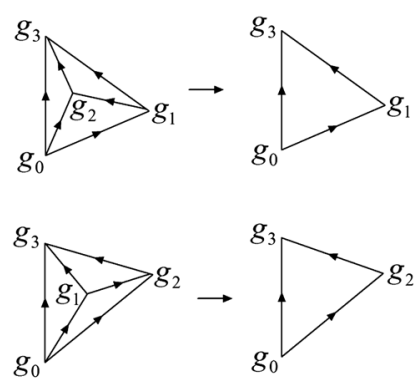

(b)

FIG. 16. All possible 2D (2-2) and (3-1) moves that admit a branching structure.
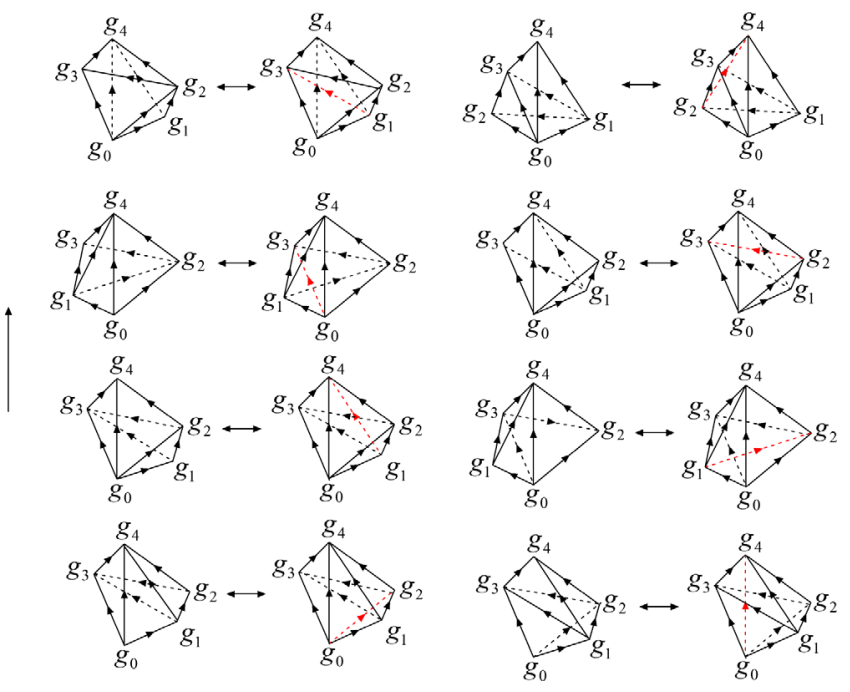

FIG. 17. All possible 3D (2-3) moves that admit a branching structure.
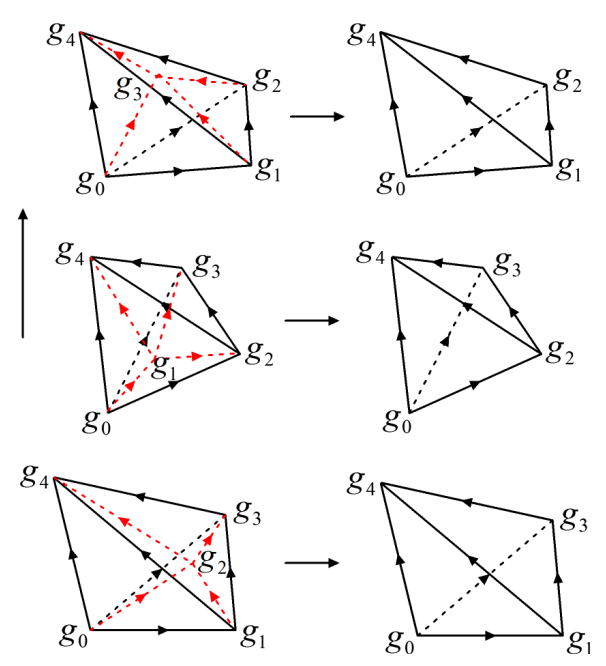

FIG. 18. All possible 3D (4-1) moves that admit a branching structure. 


\section{APPENDIX B: 2D AND 3D MOVES THAT ADMIT A BRANCHING STRUCTURE}

In this appendix, we list all possible 2D (2-2) and (3-1) moves that admit a branching structure (see Fig. 16). The 3D (2-3) and (4-1) moves that admit a branching structure are shown in Figs. 17 and 18. The arrow on the left-hand side of the figures indicates the time direction.

\section{APPENDIX C: KASTELEYN ORIENTATIONS}

To decorate Majorana fermions on the 2D or 3D triangulation lattices, we need (local) Kasteleyn orientations to design the pairing directions between the Majorana fermions. In the following, we discuss briefly how to construct (local) Kasteleyn orientations from arbitrary triangulation of the spatial manifold. We refer the interested reader to Ref. [53] for more details.

The (local) Kasteleyn orientations are constructed for arbitrary triangulation $\mathcal{T}$ of a spin manifold (spatial manifold with dimension $d$ ) with vanishing second StiefelWhitney cohomology class $\left[w^{2}\right]=[0]$. Using the mathematical result of Ref. [84], we know the representative on arbitrary triangulation of the Stiefel-Whitney homology class $w_{d-2}$, which is the Poincare dual of $w^{2}$. Then, we can construct a resolved dual lattice $\tilde{\mathcal{P}}$ with (local) Kasteleyn oriented links.

The procedure of constructing (local) Kasteleyn orientations is as follows [53]:

(1) Given a (black) triangulation lattice $\mathcal{T}$ with branching structure for a $d$-dimensional spatial spin manifold,

(2) construct the (red) resolved dual lattice $\tilde{\mathcal{P}}$, which is merely the lattice of Majorana fermions. The (red) link orientations are constructed rules related to the Stiefel-Whitney homology class $w_{d-2}$ in Ref. [84].

(3) Find the expression of $w_{d-2}$ as a formal summation of singular $(d-2)$-simplices (i.e., non-Kasteleynoriented simplices) of $\mathcal{T}$ by Ref. [84].

(4) Connect singular $(d-2)$-simplices in $\mathcal{T}$ by (blue) $(d-1)$-simplices $S$ (i.e., $\left.\partial S=w_{d-2}\right)$.

(5) Reverse the orientations of (red) links dual to (blue) $(d-1)$-simplices belonging to $S$.

(vi) Now, all the $(d-2)$-simplices in $\mathcal{T}$ are Kasteleyn oriented.

After all the above steps, the resolved dual lattice $\tilde{\mathcal{P}}$ now has (local) Kasteleyn orientations, meaning that the smallest (red) loop in $\tilde{\mathcal{P}}$ around each $(d-2)$-simplex in $\mathcal{T}$ is Kasteleyn oriented.

Using the above procedure for the special 2D and 3D triangulations, we obtain the (local) Kasteleyn orientation assignment for links inside a triangle and a tetrahedron shown in Figs. 2 and 9, respectively. All the Majorana fermions inside the standard triangle or tetrahedron are paired according to these (local) Kasteleyn orientations.

\section{APPENDIX D: CHECKING $\boldsymbol{U}(1)$ COEFFICIENT OBSTRUCTIONS BY BOCKSTEIN HOMOMORPHISM}

In solving the classification equations of FSPT states, we encounter an equation of the last layer such as

$$
d \nu_{k-1}=(-1)^{f_{k}}=e^{2 \pi i\left(f_{k} / 2\right)},
$$

where $f_{k} \in H^{k}\left(G_{b}, \mathbb{Z}_{2}\right)$ is a $\mathbb{Z}_{2}$-valued $k$-cocycle. This equation has no solution for $\nu_{k-1}$, iff $e^{2 \pi i\left(f_{k} / 2\right)}$ is a nontrivial $U(1)$-valued cocycle in $H^{k}\left[G_{b}, U(1)\right]$.

It is well known that $H^{k}\left(G_{b}, \mathbb{R} / \mathbb{Z}\right) \cong H^{k+1}\left(G_{b}, \mathbb{Z}\right)$, which comes from the short exact sequence $0 \rightarrow \mathbb{Z} \rightarrow$ $\mathbb{R} \stackrel{\bmod 1}{\rightarrow} \mathbb{R} / \mathbb{Z} \rightarrow 0$ and the condition $H^{k}\left(G_{b}, \mathbb{R}\right)=0$ for finite discrete or compact Lie group $G_{b}$. The isomorphism is given by the connecting homomorphism of the long exact sequence, which is basically the differential operator $d$ acting on the $\mathbb{R} / \mathbb{Z}$-valued cocycles. According to this isomorphism, $(-1)^{f_{k}}=e^{2 \pi i\left(f_{k} / 2\right)}$ is a nontrivial $U(1)$ valued $k$-cocycle iff $\beta\left(f_{k}\right):=d f_{k} / 2$ is a nontrivial $U(1)$-valued $(k+1)$-cocycle. Here, $\beta$ is the Bockstein homomorphism, which is the connecting homomorphism from the short exact sequence $0 \rightarrow \mathbb{Z} \stackrel{\times 2}{\rightarrow} \underset{\mathbb{Z}}{\bmod 2} \mathbb{Z}_{2} \rightarrow 0$. It maps a $\mathbb{Z}_{2}$-valued $k$-cocycle to a $\mathbb{Z}$-valued $(k+1)$-cocycle.

In summary, in order to check the last layer obstruction, we can investigate the Bockstein homomorphism of the $\mathbb{Z}_{2^{-}}$ valued obstruction functions:

$$
(-1)^{f_{k}} \in B^{k}\left[G_{b}, U(1)\right] \Leftrightarrow \beta\left(f_{k}\right) \in B^{k+1}\left(G_{b}, \mathbb{Z}\right) .
$$

In many cases, the Bockstein homomorphism of $f_{k}$ is easy to check with the identity

$$
\beta\left(f_{k}\right)=\frac{d f_{k}}{2} .
$$

The mod 2 value of Bockstein homomorphism is also related to the Steenrod square as

$$
S q^{1}\left(f_{k}\right)=f_{k} \smile_{k-1} f_{k}=\beta\left(f_{k}\right) \quad(\bmod 2) .
$$

All the above discussions can be easily generalized to the obstruction functions such as $i^{f_{k}}=e^{2 \pi i\left(f_{k} / 4\right)}$ and the $\mathbb{Z}_{T}$ rather than $\mathbb{Z}$ coefficient.

The above result can be also understood from the perspective of the universal coefficient theorem. We can use this theorem to obtain the $\mathbb{Z}_{2}$ coefficient cohomology groups from the $\mathbb{Z}$ coefficient ones:

$$
\begin{aligned}
H^{k}\left(G_{b}, \mathbb{Z}_{2}\right)= & {\left[H^{k}\left(G_{b}, \mathbb{Z}\right) \otimes_{\mathbb{Z}} \mathbb{Z}_{2}\right] } \\
& \oplus \operatorname{Tor}_{1}^{\mathbb{Z}}\left[H^{k+1}\left(G_{b}, \mathbb{Z}\right), \mathbb{Z}_{2}\right] .
\end{aligned}
$$

The above equation splits $H^{k}\left(G_{b}, \mathbb{Z}_{2}\right)$ into two types of cocycles: The first type is obtained from a $\mathbb{Z}$-valued 
$k$-cocycle by mod 2 ; the second type is obtained from a $\mathbb{Z}$ valued $(k+1)$-cocycle by basically the inverse of Bockstein homomorphism. So $(-1)^{f_{k}}$ is a trivial $U(1)$ valued cocycle iff $f_{k}$ is the first type $\mathbb{Z}_{2}$-valued cocycle (or, equivalently, $\left[\beta\left(f_{k}\right)\right]=[0]$ ).

\section{APPENDIX E: FSPT EXAMPLES}

In this appendix, we give the classifications for FSPT phases for some symmetry groups. Some simple results are summarized in Table III.

\section{2D FSPT phases for arbitrary unitary finite Abelian group}

Let us consider the arbitrary unitary finite Abelian symmetry group:

$$
G_{f}=\mathbb{Z}_{2 N_{0}}^{f} \times \prod_{i=1}^{K} \mathbb{Z}_{N_{i}} .
$$

We show below that our general group supercohomology theory gives the same classification result as Ref. [13].

The symmetry group $G_{f}$ is a central extension of the bosonic unitary finite Abelian group $G_{b}=\prod_{i=0}^{K} \mathbb{Z}_{N_{i}}$ by $\mathbb{Z}_{2}^{f}$ with the nontrivial $\left(N_{0} \geq 2\right)$ 2-cocycle

$$
\omega_{2}(a, b)=\left\lfloor\frac{a_{0}+b_{0}}{N_{0}}\right\rfloor,
$$

where we use $a=\left(a_{0}, a_{1}, \ldots, a_{K}\right)$ with $0 \leq a_{i} \leq N_{i}-1$ to denote the elements in the additive Abelian group $G_{b}$. The notation $\lfloor x\rfloor$ means the greatest integer less than or equal to $x$. If $N_{0}$ is odd, we have $\left[\omega_{2}\right]=[0]$ in $H^{2}\left(G_{b}, \mathbb{Z}_{2}\right)$, and the fermionic symmetry group $G_{f}$ is merely the direct product of $\mathbb{Z}_{2}^{f}$ and $G_{b}=\prod_{i=0}^{K} \mathbb{Z}_{N_{i}}$. We can treat both $N_{0}$ odd and $N_{0}$ even at one time in the following. Note that we use a different notation for $N_{0}$ compared to Ref. [13], where the fermionic symmetry group is chosen to be $G_{f}=\mathbb{Z}_{N_{0}}^{f} \times \prod_{i=1}^{K} \mathbb{Z}_{N_{i}}$.

Before calculating the classifications, we first list the relevant cohomology groups for $G_{b}$ with different coefficients:

$$
\begin{gathered}
H^{1}\left(G_{b}, \mathbb{Z}_{2}\right)=\prod_{0 \leq i \leq K} \mathbb{Z}_{\mathrm{gcd}\left(2, N_{i}\right)}, \\
H^{2}\left(G_{b}, \mathbb{Z}_{2}\right)=\prod_{0 \leq i \leq K} \mathbb{Z}_{\mathrm{gcd}\left(2, N_{i}\right)} \prod_{0 \leq i<j \leq K} \mathbb{Z}_{\mathrm{gcd}\left(2, N_{i j}\right)}, \\
H^{3}\left[G_{b}, U(1)_{T}\right]=\prod_{0 \leq i \leq K} \mathbb{Z}_{N_{i}} \prod_{0 \leq i<j \leq K} \mathbb{Z}_{N_{i j}} \prod_{0 \leq i<j<k \leq K} \mathbb{Z}_{N_{i j k},}
\end{gathered}
$$

$$
\begin{aligned}
& H^{4}\left[G_{b}, U(1)_{T}\right] \\
& \quad=\prod_{0 \leq i<j \leq K} \mathbb{Z}_{N_{i j}}^{2} \prod_{0 \leq i<j<k \leq K} \mathbb{Z}_{N_{i j k}}^{2} \prod_{0 \leq i<j<k<l \leq K} \mathbb{Z}_{N_{i j k l} .} .
\end{aligned}
$$

Here, $\operatorname{gcd}(x, y)$ denotes the greatest common divisor of $x$ and $y$. And $N_{i j \ldots k}$ means the greatest common divisor of $N_{i}, N_{j}, \ldots$, and $N_{k}$. One can show the above results using the Künneth formula and the universal coefficient theorem for group cohomology. Note that $N_{i}$ should be even (which we assume in the following calculations); otherwise, there is no nontrivial $\mathbb{Z}_{2}$-valued cocycle associated with the subgroup $\mathbb{Z}_{N_{i}}$.

There are also "canonical" forms for the cocycles in the cohomology groups above. To calculate the obstructions, we consider the $\mathbb{Z}_{2}$-valued cocycles:

$$
\begin{aligned}
& n_{1}^{(i)}(a)=a_{i} \quad(\bmod 2), \\
& n_{2}^{(i)}(a, b)=\left\lfloor\frac{a_{i}+b_{i}}{N_{i}}\right\rfloor,
\end{aligned}
$$

$$
n_{2}^{(i, j)}(a, b)=\left(n_{1}^{(i)} \smile n_{1}^{(j)}\right)(a, b)=a_{i} b_{j} \quad(\bmod 2),
$$

where $0 \leq i \leq K$ for the first two cocycles and $0 \leq i<$ $j \leq K$ for the last cocycle. They exhaust all nontrivial cocycles in $H^{1}\left(G_{b}, \mathbb{Z}_{2}\right)$ and $H^{2}\left(G_{b}, \mathbb{Z}_{2}\right)$. We note that the 2-cocycle Eq. (E2) is merely $\omega_{2}=n_{2}^{(0)}$ in terms of the above notations.

\section{a. Obstructions}

Different from the Abelian symmetry group $G_{f}=$ $\mathbb{Z}_{2}^{f} \times G_{b}$, not all Kitaev chain and complex fermion decorations are possible for the symmetry group Eq. (E1). We should calculate the obstructions for each layers, using the consistency equations shown in Eq. (14) (see also Table II). Since we do not consider the invertible topological order $p+i p$ superconductors as FSPT states, we need only to calculate the obstructions for $n_{1}$ and $n_{2}$. We use frequently the $\mathbb{Z}_{2}$ and $U(1)$-valued cocycle invariants in Refs. $[16,85,86]$ to check whether a cocycle is trivial or not for finite Abelian groups.

(1) Obstructions for $n_{1}$.-From Eq. (14), the obstruction function for $n_{1}$ is $\mathcal{O}_{3}\left[n_{1}\right]=\omega_{2} \smile n_{1}$ because of $s_{1}=0$. If we choose $n_{1}=n_{1}^{(i)} \quad(0 \leq i \leq K)$, the obstruction function is

$$
\mathcal{O}_{3}\left[n_{1}\right]=\omega_{2} \smile n_{1}=n_{2}^{(0)} \smile_{1}^{(i)}
$$

It is known for finite Abelian groups that the above equation is always a nontrivial cocycle if both $n_{2}^{(0)}$ and $n_{1}^{(i)}$ are nontrivial cocycles. 
Therefore, if $N_{0}$ is odd, all $n_{1}$ in Eq. (E3) are obstructionfree, and the Kitaev chain decorations are possible. If $N_{0}$ is even $\left(G_{f}\right.$ is a nontrivial central extension of $\left.G_{b}\right)$, all nontrivial $n_{1}$ in Eq. (E3) are obstructed, and the Kitaev chain decorations are illegal. The (cohomology class) number of obstruction-free $n_{1}$ is

$$
\#\left(\text { obstruction-free } n_{1}\right)=\prod_{1 \leq i \leq K} \frac{\operatorname{gcd}\left(2, N_{i}\right)}{\operatorname{gcd}\left(2, N_{0 i}\right)}
$$

(2) Obstructions for $n_{2}$.-From Eq. (14) [see also Eq. (136)], the obstruction function for $n_{2}$ is

$$
\mathcal{O}_{4}\left[n_{2}\right]=(-1)^{n_{2} \smile n_{2}+\omega_{2} \smile n_{2}},
$$

which is a cocycle in $H^{4}\left(G_{b}, U(1)_{T}\right)$. Note that, if $N_{0}$ is odd, we can choose $\omega_{2}=0$, and the equation for $n_{2}$ is always $d n_{2}=0$. So many terms in Eq. (136) vanish, including the $( \pm i)$ terms. Since there are many different types of $n_{2}$ in Eq. (E4), we discuss them separately.

(2.1) $n_{2}=n_{2}^{(0)}$. - Since $\omega_{2}=n_{2}^{(0)}$, the obstruction function Eq. (E12) is always 1. So this $n_{2}$ is obstruction-free.

(2.2) $n_{2}=n_{2}^{(i)}(1 \leq i \leq K)$. - Using the $U(1)$-valued cocycle invariants, it is easy to check that both $(-1)^{n_{2}} \smile n_{2}$ and $(-1)^{\omega_{2} \smile n_{2}}$ are $U(1)$-valued coboundaries. So this $n_{2}$ is also obstruction-free.

(2.3) $n_{2}=n_{2}^{(0, i)}(1 \leq i \leq K)$. - In this case, the cocycle $(-1)^{\omega_{2} \smile n_{2}}=(-1)^{n_{2}^{(0)} \smile n_{2}^{(0, i)}}=(-1)^{n_{2}^{(0)} \smile n_{1}^{(0)} \smile n_{1}^{(i)}}$ is nontrivial. And the other part $(-1)^{n_{2} \smile n_{2}}=(-1)^{n_{2}^{(0, i)} \smile n_{2}^{(0, i)}}$ is still a 4-coboundary. So these nontrivial $n_{2}$ are always obstructed.

(2.4) $n_{2}=n_{2}^{(i, j)}(1 \leq i<j \leq K)$. - In this case, the obstruction function is

$$
\begin{aligned}
& \mathcal{O}_{4}\left[n_{2}\right]=(-1)^{n_{2} \smile n_{2}+\omega_{2} \smile n_{2}}
\end{aligned}
$$

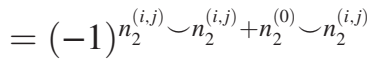

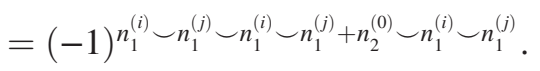

By calculating the $U(1)$-valued cocycle invariants, the first

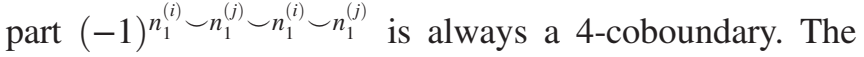
second part $(-1)^{n_{2}^{(0)} \smile n_{1}^{(i)} \smile n_{1}^{(j)}}$ is a 4-coboundary if and only if $N_{i j} / N_{0 i j}$ is even. So $n_{2}^{(i, j)}(1 \leq i<j \leq K)$ is obstructionfree if and only if $N_{i j} / N_{0 i j}$ is even.

Summarizing the above results for $n_{2}$, the total number of obstruction-free $n_{2}$ is

$$
\begin{aligned}
& \#\left(\text { obstruction-free } n_{2}\right) \\
& =\prod_{0 \leq i \leq K} \operatorname{gcd}\left(2, N_{i}\right) \prod_{1 \leq i<j \leq K} \operatorname{gcd}\left(2, \frac{N_{i j}}{N_{0 i j}}\right) .
\end{aligned}
$$

\section{b. Trivializations}

(1) Trivializations of $n_{2}$.-According to the trivialized subgroups Eq. (15) for 2D FSPT states, the 2-cocycle $n_{2}$ in the $\Gamma^{2}$ is trivialized by 1D ASPT states on the boundary. It is discussed in detail in Sec. VE. For the 2-cocycle $\omega_{2}=n_{2}^{(0)}$, we have $\Gamma^{2}=\left\{\omega_{2} \smile n_{0} \in H^{2}\left(G_{b}, \mathbb{Z}_{2}\right) \mid n_{0} \in\right.$ $\left.H^{0}\left(G_{b}, \mathbb{Z}_{2}\right)\right\}=\left\langle n_{2}^{(0)}\right\rangle$. The complex fermion decoration data $n_{2}=n_{2}^{(0)}$ are trivialized. (Note that this $n_{2}$ is not obstructed as discussed above.) So the first subgroup $\mathbb{Z}_{\operatorname{gcd}\left(2, N_{0}\right)}$ of $H^{2}\left(G_{b}, \mathbb{Z}_{2}\right)$ in Eq. (E4) does not correspond to a nontrivial 2D FSPT state.

Combining the trivializations of $n_{2}$ with obstruction-free $n_{2}$ number Eq. (E14), the number of trivialization-free obstruction-free $n_{2}$ is

$$
\begin{aligned}
& \left.\# \text { (trivialization-free obstruction-free } n_{2}\right) \\
& =\prod_{1 \leq i \leq K} \operatorname{gcd}\left(2, N_{i}\right) \prod_{1 \leq i<j \leq K} \operatorname{gcd}\left(2, \frac{N_{i j}}{N_{0 i j}}\right) .
\end{aligned}
$$

(2) Trivializations of $\nu_{3}$. - For the bosonic $U(1)$ phase factor $\nu_{3}$, the trivialized subgroup in Eq. (15) can be calculated as

$$
\begin{aligned}
\Gamma^{3} & =\left\{(-1)^{\omega_{2} \smile n_{1}} \in H^{3}\left[G_{b}, U(1)_{T}\right] \mid n_{1} \in H^{1}\left(G_{b}, \mathbb{Z}_{2}\right)\right\} \\
& =\left\langle(-1)^{n_{2}^{(0)}} \sim_{1}^{(i)} \mid 0 \leq i \leq K, \operatorname{gcd}\left(2, N_{i}\right)=2\right\rangle .
\end{aligned}
$$

For the cocycles in the subgroup $\mathbb{Z}_{N_{0}} \times \prod_{1 \leq i \leq K} \mathbb{Z}_{0 i}$ of $H^{3}\left[G_{b}, U(1)_{T}\right]$ in Eq. (E5), they have a "canonical" form expressed as lower-dimensional $\mathbb{Z}_{2}$-valued cocycles $n_{1}^{(i)}$ $(0 \leq i \leq K)$ and $n_{2}^{(0)}$ as (only for even $N_{i}$; otherwise, the cocycle $n_{1}^{(i)}$ is trivial)

$$
\nu_{3}=e^{2 \pi i\left(k / N_{i}\right) n_{2}^{(0)} \smile n_{1}^{(i)}}\left(k=0,1, \ldots, N_{0 i}-1\right) .
$$

The generating cocycle of $\Gamma^{3}$ in Eq. (E16) can be expressed as $(-1)^{n_{2}^{(0)} \smile n_{1}^{(i)}}=e^{2 \pi i\left(N_{i} / 2 / N_{i}\right) n_{2}^{(0)} \smile n_{1}^{(i)}}$. Comparing it with the above equation, we see that if $N_{i}$ is odd, or $N_{i} / 2$ is an integral multiplier of $N_{0 i}$, then the part of $\Gamma^{2}$ related to $\mathbb{Z}_{N_{i}} \subset G_{b} \quad(0 \leq i \leq K) \quad$ is trivial in $H^{3}\left[G_{b}, U(1)_{T}\right]$. Otherwise, the 3-cocycle $\nu_{3}$ in Eq. (E17) with $k=N_{i} / 2$ $\left(\bmod N_{0 i}\right)$ is trivialized by the 2D ASPT state. The results calculated above can also be obtained from calculating the cocycle invariants for $\nu_{3}[16,85,86]$.

In summary, (1) if $N_{0}$ is even, one nontrivial $\nu_{3}$ in $\mathbb{Z}_{N_{0}} \subset$ $H^{3}\left[G_{b}, U(1)_{T}\right]$ is trivialized. Otherwise, all elements in $\mathbb{Z}_{N_{0}} \subset H^{3}\left[G_{b}, U(1)_{T}\right]$ are nontrivial. (2) For $1 \leq i \leq K$, and the subgroup $\mathbb{Z}_{N_{0 i}} \subset H^{3}\left[G_{b}, U(1)_{T}\right]$, we also have two possibilities. If $N_{i}$ is even and $N_{i} / N_{0 i}$ is odd [87], then one nontrivial $\nu_{3}$ in $\mathbb{Z}_{N_{0 i}} \subset H^{3}\left[G_{b}, U(1)_{T}\right]$ is trivialized. Otherwise, all elements in $\mathbb{Z}_{N_{0 i}} \subset H^{3}\left[G_{b}, U(1)_{T}\right]$ are 
nontrivial. So the number of trivialization-free cocycles in the subgroup $\mathbb{Z}_{N_{0}} \times \prod_{1 \leq i \leq K} \mathbb{Z}_{0 i} \subset H^{3}\left[G_{b}, U(1)_{T}\right]$ in Eq. (E5) is

$$
\frac{N_{0}}{\operatorname{gcd}\left(2, N_{0}\right)} \prod_{1 \leq i \leq K} \frac{\operatorname{gcd}\left(2 N_{0}, N_{i}\right)}{\operatorname{gcd}\left(2, N_{i}\right)} .
$$

The total number of $\nu_{3}$ that is not trivialized in Eq. (E5) is

$$
\begin{aligned}
& \#\left(\text { trivialization-free } \nu_{3}\right) \\
& =\frac{N_{0}}{\operatorname{gcd}\left(2, N_{0}\right)} \prod_{1 \leq i \leq K} N_{i} \\
& \quad \cdot \frac{\operatorname{gcd}\left(2 N_{0}, N_{i}\right)}{\operatorname{gcd}\left(2, N_{i}\right)} \prod_{1 \leq i<j \leq K} N_{i j} \prod_{0 \leq i<j<k \leq K} N_{i j k} .
\end{aligned}
$$

\section{c. Full classification}

From the above calculations of obstructions and trivializations, we can obtain the number of 2D FSPT phases with symmetry group Eq. (E1) by combining Eqs. (E11), (E15), and (E19):

$$
\begin{aligned}
\#(\mathrm{FSPT})= & \frac{N_{0}}{\operatorname{gcd}\left(2, N_{0}\right)} \prod_{1 \leq i \leq K} N_{i} \cdot \frac{\operatorname{gcd}\left(2, N_{i}\right) \cdot \operatorname{gcd}\left(2 N_{0}, N_{i}\right)}{\operatorname{gcd}\left(2, N_{0 i}\right)} \\
& \times \prod_{1 \leq i<j \leq K} N_{i j} \cdot \operatorname{gcd}\left(2, \frac{N_{i j}}{N_{0 i j}}\right) \prod_{0 \leq i<j<k \leq K} N_{i j k} .
\end{aligned}
$$

If $N_{0}$ is even, the above equation is reduced to

$$
\begin{aligned}
\left.\#(\mathrm{FSPT})\right|_{N_{0} \text { even }}= & \frac{N_{0}}{2} \prod_{1 \leq i \leq K} N_{i} \cdot \operatorname{gcd}\left(2 N_{0}, N_{i}\right) \\
& \times \prod_{1 \leq i<j \leq K} N_{i j} \cdot \operatorname{gcd}\left(2, \frac{N_{i j}}{N_{0 i j}}\right) \prod_{0 \leq i<j<k \leq K} N_{i j k} .
\end{aligned}
$$

On the other hand, if $N_{0}$ is odd, we have

$$
\begin{aligned}
\left.\#(\mathrm{FSPT})\right|_{N_{0} \text { odd }}= & N_{0} \prod_{1 \leq i \leq K} N_{i} \cdot \operatorname{gcd}\left(2, N_{i}\right) \\
& \cdot \operatorname{gcd}\left(2 N_{0}, N_{i}\right) \prod_{1 \leq i<j \leq K} N_{i j} \\
& \cdot \operatorname{gcd}\left(2, N_{i j}\right) \prod_{0 \leq i<j<k \leq K} N_{i j k} .
\end{aligned}
$$

After some calculations [88], one can show that the above two equations for the number of 2D FSPT phases are exactly the same as Eqs. (42) and (43) of Ref. [13], which are obtained from a totally different approach of the braiding statistics data of the gauge flux. Furthermore, one can show that the group structure of general group supercohomology classification [89] also agrees with Ref. [13]. We note again that we use a different convention of $N_{0}$ definition compared to Ref. [13].

\section{Four symmetry groups with $G_{b} \cong \mathbb{Z}_{2}$ and different $s_{1}$ and $\omega_{2}$}

If we focus on the bosonic symmetry group $G_{b}$ that is isomorphic to $\mathbb{Z}_{2}=\{0,1\}$, there are in total four different $G_{f}$ with different choices of cocycles $s_{1}$ and $\omega_{2}$. It is already discussed briefly at the end of Sec. IB. The nontrivial cocycles in $H^{1}\left(\mathbb{Z}_{2}, \mathbb{Z}_{2}\right)=\mathbb{Z}_{2}$ and $H^{2}\left(\mathbb{Z}_{2}, \mathbb{Z}_{2}\right)=\mathbb{Z}_{2}$ are

$$
s_{1}(a)= \begin{cases}1, & a=1, \\ 0, & \text { others }\end{cases}
$$

$$
\omega_{2}(a, b)= \begin{cases}1, & a=b=1, \\ 0, & \text { others. }\end{cases}
$$

The classifications of FSPT phases for these groups are given in Table III. For convenience, we resummarize them in Table VI.

We note that we do not consider the invertible topological order (such as a Kitaev chain in 1D) as a FSPT state, since they do not need any bosonic symmetry protection ( $\mathbb{Z}_{2}^{f}$ can never be broken). If we include them to consider invertible phases, the classification results are given in Table VII. There is an additional $\mathbb{Z}_{2}$ subgroup for $G_{f}=\mathbb{Z}_{2}^{f} \times \mathbb{Z}_{2}$ and $G_{f}=\mathbb{Z}_{2}^{f} \times \mathbb{Z}_{2}^{T}$ in $1 \mathrm{D}$ (obstructed if $\omega_{2}$ is nontrivial). It corresponds to the $n_{0}$ data of $1 \mathrm{D}$ invertible topological order of Kitaev chain. There is an additional

$\mathbb{Z}$ classification for symmetry group $G_{f}=\mathbb{Z}_{2}^{f} \times \mathbb{Z}_{2}$ and $G_{f}=\mathbb{Z}_{4}^{f}$ in 2D. It corresponds to the several layers of 2D $p+i p$ chiral superconductors as fermionic invertible topological orders (the root states of the two $\mathbb{Z}$ classifications are different). It is not possible if there is time-reversal symmetry in $G_{b}$ [for $H^{0}\left(G_{b}, \mathbb{Z}_{T}\right)=0$ ]. We note that all the results are consistent with the spin cobordism calculations [10].

TABLE VI. Classification of FSPT phases with $G_{b} \cong \mathbb{Z}_{2}$ and different choices of $s_{1}$ and $\omega_{2}$.

\begin{tabular}{lcccc}
\hline \hline$G_{f} \backslash \operatorname{dim}$ & 0 & 1 & 2 & 3 \\
\hline $\mathbb{Z}_{2}^{f} \times \mathbb{Z}_{2}$ & $\mathbb{Z}_{2} \times \mathbb{Z}_{2}$ & $\mathbb{Z}_{2}$ & $\mathbb{Z}_{8}$ & $\mathbb{Z}_{1}$ \\
$\mathbb{Z}_{4}^{f}=\mathbb{Z}_{2}^{f} \times{ }_{\omega_{2}} \mathbb{Z}_{2}$ & $\mathbb{Z}_{4}$ & $\mathbb{Z}_{1}$ & $\mathbb{Z}_{1}$ & $\mathbb{Z}_{1}$ \\
$\mathbb{Z}_{2}^{f} \times \mathbb{Z}_{2}^{T}$ & $\mathbb{Z}_{2}$ & $\mathbb{Z}_{4}$ & $\mathbb{Z}_{1}$ & $\mathbb{Z}_{1}$ \\
$\mathbb{Z}_{4}^{T f}=\mathbb{Z}_{2}^{f} \times{ }_{\omega_{2}} \mathbb{Z}_{2}^{T}$ & $\mathbb{Z}_{1}$ & $\mathbb{Z}_{2}$ & $\mathbb{Z}_{2}$ & $\mathbb{Z}_{16}$ \\
\hline \hline
\end{tabular}


TABLE VII. Classification of fermionic invertible phases with $G_{b} \cong \mathbb{Z}_{2}$ and different choices of $s_{1}$ and $\omega_{2}$. The differences compared to Table VI (with fermionic invertible topological orders included) are emphasized by bold.

\begin{tabular}{lcccc}
\hline \hline$G_{f} \backslash \operatorname{dim}$ & 0 & 1 & 2 & 3 \\
\hline $\mathbb{Z}_{2}^{f} \times \mathbb{Z}_{2}$ & $\mathbb{Z}_{2} \times \mathbb{Z}_{2}$ & $\mathbb{Z}_{2} \times \mathbb{Z}_{2}$ & $\mathbb{Z} \times \mathbb{Z}_{8}$ & $\mathbb{Z}_{1}$ \\
$\mathbb{Z}_{4}^{f}=\mathbb{Z}_{2}^{f} \times_{\omega_{2}} \mathbb{Z}_{2}$ & $\mathbb{Z}_{4}$ & $\mathbb{Z}_{1}$ & $\mathbb{Z}$ & $\mathbb{Z}_{1}$ \\
$\mathbb{Z}_{2}^{f} \times \mathbb{Z}_{2}^{T}$ & $\mathbb{Z}_{2}$ & $\mathbb{Z}_{8}$ & $\mathbb{Z}_{1}$ & $\mathbb{Z}_{1}$ \\
$\mathbb{Z}_{4}^{T f}=\mathbb{Z}_{2}^{f} \times{ }_{\omega_{2}} \mathbb{Z}_{2}^{T}$ & $\mathbb{Z}_{1}$ & $\mathbb{Z}_{2}$ & $\mathbb{Z}_{2}$ & $\mathbb{Z}_{16}$ \\
\hline \hline
\end{tabular}

$$
\text { a. } G_{f}=\mathbb{Z}_{2}^{f} \times \mathbb{Z}_{2}
$$

(1) OD. - The classification data are $\left(n_{0}, \nu_{1}\right) \in$ $H^{0}\left(G_{b}, \mathbb{Z}_{2}\right) \times H^{1}\left[G_{b}, U(1)_{T}\right]=\mathbb{Z}_{2} \times \mathbb{Z}_{2}$. There are no obstructions and trivializations for these data. In another approach, the classification of one-dimensional irreducible representations for $G_{f}$ is $H^{1}\left[\mathbb{Z}_{2}^{f} \times \mathbb{Z}_{2}, U(1)_{T}\right]=\mathbb{Z}_{2} \times \mathbb{Z}_{2}$. So the two approaches agree with each other, and the 0D FSPT phases are classified by $\mathbb{Z}_{2} \times \mathbb{Z}_{2}$.

(2) 1D.-The classification data are $\left(n_{1}, \nu_{2}\right) \in$ $H^{1}\left(G_{b}, \mathbb{Z}_{2}\right) \times H^{2}\left[G_{b}, U(1)_{T}\right]=\mathbb{Z}_{2} \times \mathbb{Z}_{1}$. There are no obstructions or trivializations.

(3) $2 D$. - The classification data are $\left(n_{1}, n_{2}, \nu_{3}\right) \in$ $H^{1}\left(G_{b}, \mathbb{Z}_{2}\right) \times H^{2}\left(G_{b}, \mathbb{Z}_{2}\right) \times H^{3}\left[G_{b}, U(1)_{T}\right]=\mathbb{Z}_{2} \times \mathbb{Z}_{2} \times \mathbb{Z}_{2}$. There are no obstructions or trivializations. And the group structure of the classification [89] can be shown to be $\mathbb{Z}_{8}$. So two copies of Kitaev chain decoration states give the complex fermion decoration state. Two copies of complex fermion decoration states give the nontrivial BSPT state. And, finally, two copies of BSPT states are trivial. This classification of $2 \mathrm{D}$ topological superconductors with $\mathbb{Z}_{2}$ Ising symmetry is first obtained in Ref. [47].

(4) $3 D$.- The classification data are $\left(n_{1}, n_{2}, n_{3}, \nu_{4}\right) \in$ $H^{1}\left(G_{b}, \mathbb{Z}\right) \times H^{2}\left(G_{b}, \mathbb{Z}_{2}\right) \times H^{3}\left(G_{b}, \mathbb{Z}_{2}\right) \times H^{4}\left[G_{b}, U(1)_{T}\right]=$ $\mathbb{Z}_{1} \times \mathbb{Z}_{2} \times \mathbb{Z}_{2} \times \mathbb{Z}_{1}$. One can show that the nontrivial cocycles satisfy $n_{2} \smile n_{2} \notin B^{4}\left(G_{b}, \mathbb{Z}_{2}\right)$ and $(-1)^{n_{3} \smile_{1} n_{3}} \notin$ $B^{5}\left[G_{b}, U(1)_{T}\right]$. According to the consistency equations in Table II, all states are obstructed. There is only one trivial phase.

$$
\text { b. } G_{f}=\mathbb{Z}_{4}^{f}=\mathbb{Z}_{2}^{f} \times_{\omega_{2}} \mathbb{Z}_{2}
$$

(1) OD.- In terms of our classification data, we have $\left(n_{0}, \nu_{1}\right) \in H^{0}\left(G_{b}, \mathbb{Z}_{2}\right) \times H^{1}\left[G_{b}, U(1)_{T}\right]=\mathbb{Z}_{2} \times \mathbb{Z}_{2}$. And both data are obstruction-free. In another way, the one-dimensional irreducible representation for $G_{f}$ is classified by $H^{1}\left[\mathbb{Z}_{4}^{f}, U(1)_{T}\right]=\mathbb{Z}_{4}$. So the classification is $\mathbb{Z}_{4}$.

(2) 1D.-The classification data are $\left(n_{1}, \nu_{2}\right) \in$ $H^{1}\left(G_{b}, \mathbb{Z}_{2}\right) \times H^{2}\left[G_{b}, U(1)_{T}\right]=\mathbb{Z}_{2} \times \mathbb{Z}_{1}$. However, the nontrivial $n_{1}$ is obstructed. So there is only one trivial phase.
(3) $2 D$. - The classification data are $\left(n_{1}, n_{2}, \nu_{3}\right) \in$ $H^{1}\left(G_{b}, \mathbb{Z}_{2}\right) \times H^{2}\left(G_{b}, \mathbb{Z}_{2}\right) \times H^{3}\left[G_{b}, U(1)_{T}\right]=\mathbb{Z}_{2} \times \mathbb{Z}_{2} \times \mathbb{Z}_{2}$. The nontrivial $n_{1}$ is obstructed. As discussed in detail in Sec. V E, the nontrivial $n_{2}$ is trivialized by the boundary 1D ASPT (recall the obstruction of Kitaev chain layer $n_{0}$ in 1D). And the nontrivial cocycle $\nu_{3}$ is also trivialized (recall the obstruction of $n_{1}$ in $\left.1 \mathrm{D}\right)$. So the classification is $\mathbb{Z}_{1}$, which is consistent with Refs. [13,56].

(4) $3 D$. - The classification data are $\left(n_{1}, n_{2}, n_{3}, \nu_{4}\right) \in$ $H^{1}\left(G_{b}, \mathbb{Z}\right) \times H^{2}\left(G_{b}, \mathbb{Z}_{2}\right) \times H^{3}\left(G_{b}, \mathbb{Z}_{2}\right) \times H^{4}\left[G_{b}, U(1)_{T}\right]=$ $\mathbb{Z}_{1} \times \mathbb{Z}_{2} \times \mathbb{Z}_{2} \times \mathbb{Z}_{1}$. The nontrivial $n_{2}$ is trivialized (recall the obstruction of $n_{0}$ in 2D). And the nontrivial $n_{3}$ is also trivialized (recall the obstruction of $n_{1}$ in 2D). So there is only one trivial phase.

$$
\text { c. } G_{f}=\mathbb{Z}_{2}^{f} \times \mathbb{Z}_{2}^{T}
$$

(1) $0 D$. - The classification data are $\left(n_{0}, \nu_{1}\right) \in$ $H^{0}\left(G_{b}, \mathbb{Z}_{2}\right) \times H^{1}\left[G_{b}, U(1)_{T}\right]=\mathbb{Z}_{2} \times \mathbb{Z}_{1}$. The cocycle $n_{0}$ is obstruction-free. In another approach, the classification of one-dimensional irreducible representations for $G_{f}$ is $H^{1}\left[\mathbb{Z}_{2}^{f} \times \mathbb{Z}_{2}^{T}, U(1)_{T}\right]=\mathbb{Z}_{2}$. So the classification is $\mathbb{Z}_{2}$.

(2) 1D.-The classification data are $\left(n_{1}, \nu_{2}\right) \in$ $H^{1}\left(G_{b}, \mathbb{Z}_{2}\right) \times H^{2}\left[G_{b}, U(1)_{T}\right]=\mathbb{Z}_{2} \times \mathbb{Z}_{2}$. There is neither obstruction nor trivialization. The classification is $\mathbb{Z}_{4}$. If we include the invertible topological order Kitaev chain, then the classification is $\mathbb{Z}_{8}$. This classification of $1 \mathrm{D}$ $T^{2}=1$ topological superconductors is first obtained in Refs. [42,43].

(3) $2 D$.- The classification data are $\left(n_{1}, n_{2}, \nu_{3}\right) \in$ $H^{1}\left(G_{b}, \mathbb{Z}_{2}\right) \times H^{2}\left(G_{b}, \mathbb{Z}_{2}\right) \times H^{3}\left[G_{b}, U(1)_{T}\right]=\mathbb{Z}_{2} \times \mathbb{Z}_{2} \times \mathbb{Z}_{1}$. The nontrivial $n_{1}$ obstructed for $s_{1} \smile n_{1} \smile n_{1}$ is nontrivial in $H^{3}\left(G_{b}, \mathbb{Z}_{2}\right)$. This obstruction is the fermion parity obstruction for $T^{2}=12 \mathrm{D}$ topological superconductors considered in Refs. $[66,76]$. The nontrivial $n_{2}$ is obstructed for $(-1)^{n_{2} \smile n_{2}} \notin B^{4}\left[G_{b}, U(1)_{T}\right]$. So there is only one trivial phase.

(4) $3 D$. - The classification data are $\left(n_{1}, n_{2}, n_{3}, \nu_{4}\right) \in$ $H^{1}\left(G_{b}, \mathbb{Z}\right) \times H^{2}\left(G_{b}, \mathbb{Z}_{2}\right) \times H^{3}\left(G_{b}, \mathbb{Z}_{2}\right) \times H^{4}\left[G_{b}, U(1)_{T}\right]=$ $\mathbb{Z}_{2} \times \mathbb{Z}_{2} \times \mathbb{Z}_{2} \times \mathbb{Z}_{2}$. The nontrivial cocycle data $n_{1}$ of $p+i p$ chiral superconductor decoration is obstructed for $s_{1} \smile n_{1} \smile n_{1} \notin B^{3}\left(G_{b}, \mathbb{Z}_{2}\right)$. The nontrivial $n_{2}$ is also obstructed, by calculating the cocycle invariants for the obstruction function $n_{2} \smile n_{2}+s_{1} \smile\left(n_{2} \smile_{1} n_{2}\right) \notin B^{4}\left(G_{b}, \mathbb{Z}_{2}\right)$. The nontrivial $n_{3}$ is trivialized by the 2D ASPT state (recall the obstruction of $n_{1}$ in 2D). And the nontrivial $\nu_{4}$ is also trivialized by another layer of the 2D ASPT state (recall the obstruction of $n_{2}$ in 2D). In summary, there is only one trivial FSPT phase.

$$
\text { d. } G_{f}=\mathbb{Z}_{4}^{T f}=\mathbb{Z}_{2}^{f} \times_{\omega_{2}} \mathbb{Z}_{2}^{T}
$$

(1) $0 D$. - The classification data are $\left(n_{0}, \nu_{1}\right) \in$ $H^{0}\left(G_{b}, \mathbb{Z}_{2}\right) \times H^{1}\left[G_{b}, U(1)_{T}\right]=\mathbb{Z}_{2} \times \mathbb{Z}_{1}$. Since the cocycle $(-1)^{\omega_{2} \smile n_{0}} \in H^{2}\left[G_{b}, U(1)_{T}\right]$ is nontrivial, the 
nontrivial $n_{0}$ is obstructed. In another approach, the classification of one-dimensional irreducible representations for $G_{f}$ is $H^{1}\left[\mathbb{Z}_{2}^{f} \times_{\omega_{2}} \mathbb{Z}_{2}^{T}, U(1)_{T}\right]=\mathbb{Z}_{1}$. So the classification is $\mathbb{Z}_{1}$. The physical meaning is that a nontrivial fermionic mode with $T^{2}=-1$ must be in a Kramers doublet, which is twofold degenerate. So there is only one trivial class.

(2) $1 D$.-The classification data are $\left(n_{1}, \nu_{2}\right) \in$ $H^{1}\left(G_{b}, \mathbb{Z}_{2}\right) \times H^{2}\left[G_{b}, U(1)_{T}\right]=\mathbb{Z}_{2} \times \mathbb{Z}_{2}$. The nontrivial $n_{1}$ is obstruction-free. The nontrivial $\nu_{2}$ is trivialized by the 0D ASPT state (recall the obstruction of $n_{0}$ in 0D). So the classification is $\mathbb{Z}_{2}$ corresponding to the complex fermion decorations.

(3) $2 D$.-The classification data are $\left(n_{1}, n_{2}, \nu_{3}\right) \in$ $H^{1}\left(G_{b}, \mathbb{Z}_{2}\right) \times H^{2}\left(G_{b}, \mathbb{Z}_{2}\right) \times H^{3}\left[G_{b}, U(1)_{T}\right]=\mathbb{Z}_{2} \times \mathbb{Z}_{2} \times \mathbb{Z}_{1}$. The first cocycle $n_{1}$ is obstruction-free, for the obstruction function is zero: $\omega_{2} \smile n_{1}+s_{1} \smile n_{1} \smile n_{1}=0$. The nontrivial cocycle data $n_{2}$ is trivialized (recall the obstruction of $n_{0}$ in 1D). In summary, there is only one nontrivial topological superconductor with $T^{2}=-1$. It is exactly the Kitaev chain decoration state constructed in Ref. [76].

(4) $3 D$.-The classification data are $\left(n_{1}, n_{2}, n_{3}, \nu_{4}\right) \in$ $H^{1}\left(G_{b}, \mathbb{Z}\right) \times H^{2}\left(G_{b}, \mathbb{Z}_{2}\right) \times H^{3}\left(G_{b}, \mathbb{Z}_{2}\right) \times H^{4}\left[G_{b}, U(1)_{T}\right]=$ $\mathbb{Z}_{2} \times \mathbb{Z}_{2} \times \mathbb{Z}_{2} \times \mathbb{Z}_{2}$. The nontrivial cocycle $n_{1}$ is obstruction-free, for the obstruction function is $\omega_{2} \smile n_{1}+s_{1} \smile$ $n_{1} \smile n_{1}=0$. The nontrivial cocycle $n_{2}$ is also obstructionfree, since the obstruction function $\omega_{2} \smile n_{2}+n_{2} \smile n_{2}+$ $s_{1} \smile\left(n_{2} \smile n_{1} n_{2}\right)=s_{1} \smile\left(n_{2} \smile_{1} n_{2}\right)$ is in $B^{4}\left(G_{b}, \mathbb{Z}_{2}\right)$. Since $H^{5}\left[G_{b}, U(1)_{T}\right]=0$, the classification data $n_{3}$ are always obstruction-free. In summary, all four layers of classification data are obstruction-free and trivialization-free. So the classification of 3D $T^{2}=-1$ topological superconductors is $\mathbb{Z}_{16}$, which is first shown by Kitaev and Morgan [90].

\section{FSPT states with quaternion group$$
\boldsymbol{G}_{f}=Q_{8}^{f}=\mathbb{Z}_{2}^{f} \times_{\omega_{2}}\left(\mathbb{Z}_{2} \times \mathbb{Z}_{2}\right)
$$

The quaternion group $Q_{8}$ is defined as $Q_{8}=\langle i, j, k| i^{2}=$ $\left.j^{2}=k^{2}=i j k\right\rangle$ with order 8 . Usually, we denote $i j k$ as -1 . Other useful relations are $i j=k, j k=i$, and $k i=j$, which can be easily derived from the definition of $Q_{8}$. Since the center of $Q_{8}$ is $\{ \pm 1\}$, we can unambiguously identify it with the fermion parity group $\mathbb{Z}_{2}^{f}$. Then, the bosonic symmetry group (as a quotient group) $G_{b}=G_{f} / \mathbb{Z}_{2}^{f}=Q_{8}^{f} / \mathbb{Z}_{2}^{f}$ is generated by $[i]$ and $[j]$ and has relations $[i]^{2}=[j]^{2}=$ $[i][j]=[1]$. So $G_{b}$ is isomorphic to $\mathbb{Z}_{2} \times \mathbb{Z}_{2}$. In terms of the short exact sequence, we have

$$
1 \rightarrow \mathbb{Z}_{2}^{f} \rightarrow Q_{8}^{f} \rightarrow \mathbb{Z}_{2} \times \mathbb{Z}_{2} \rightarrow 1 .
$$

The nontrivial 2-cocycle of the central extension is given by

$$
\omega_{2}=n_{2}^{(1)}+n_{2}^{(2)}+n_{1}^{(1)} n_{1}^{(2)},
$$

which is the most nontrivial element in $H^{2}\left(\mathbb{Z}_{2} \times \mathbb{Z}_{2}, \mathbb{Z}_{2}\right)=$ $\mathbb{Z}_{2}^{3}=\left\langle n_{2}^{(1)}, n_{2}^{(2)}, n_{1}^{(1)} n_{1}^{(2)}\right\rangle$. Here, $n_{1}^{(i)}$ and $n_{2}^{(i)}=n_{1}^{(i)} \smile n_{1}^{(i)}$ are the nontrivial 1- and 2-cocycles, respectively, for the $i$ th $(i=1,2) \mathbb{Z}_{2}$ subgroup of $G_{b}=\mathbb{Z}_{2}^{(1)} \times \mathbb{Z}_{2}^{(2)}$. The three terms of $\omega_{2}$ in Eq. (E26) indicate $i^{2}=-1, j^{2}=-1$, and $i j=-j i$, respectively, in $Q_{8}^{f}$.

We show below that there is a 3D anomaly-free FSPT state for $G_{f}=Q_{8}^{f}$ with complex fermion decoration $n_{3}=n_{2}^{(1)} \smile n_{1}^{(2)}+n_{1}^{(1)} \smile n_{2}^{(2)}$. However, this state is trivialized by $\omega_{2} \smile_{1} \omega_{2} \in \Gamma^{3}$ [see Eq. (19)], which is related to the boundary 2D ASPT state with $n_{0}=2$ copies of $p+i p$ superconductors.

The relevant cohomology groups of $G_{b}=\mathbb{Z}_{2}^{(1)} \times \mathbb{Z}_{2}^{(2)}$ with $\mathbb{Z}_{2}$ a coefficient are

$$
\begin{gathered}
H^{0}\left(\mathbb{Z}_{2} \times \mathbb{Z}_{2}, \mathbb{Z}_{2}\right)=\mathbb{Z}_{2}=\langle 1\rangle, \\
H^{1}\left(\mathbb{Z}_{2} \times \mathbb{Z}_{2}, \mathbb{Z}_{2}\right)=\mathbb{Z}_{2}^{2}=\left\langle n_{1}^{(1)}, n_{1}^{(2)}\right\rangle, \\
H^{2}\left(\mathbb{Z}_{2} \times \mathbb{Z}_{2}, \mathbb{Z}_{2}\right)=\mathbb{Z}_{2}^{3}=\left\langle n_{2}^{(1)}, n_{2}^{(2)}, n_{1}^{(1)} n_{1}^{(2)}\right\rangle, \\
H^{3}\left(\mathbb{Z}_{2} \times \mathbb{Z}_{2}, \mathbb{Z}_{2}\right)=\mathbb{Z}_{2}^{4}=\left\langle n_{3}^{(1)}, n_{2}^{(1)} n_{1}^{(2)}, n_{1}^{(1)} n_{2}^{(2)}, n_{3}^{(2)}\right\rangle,
\end{gathered}
$$

$$
\begin{aligned}
& H^{4}\left(\mathbb{Z}_{2} \times \mathbb{Z}_{2}, \mathbb{Z}_{2}\right) \\
& \quad=\mathbb{Z}_{2}^{5}=\left\langle n_{4}^{(1)}, n_{3}^{(1)} n_{1}^{(2)}, n_{2}^{(1)} n_{2}^{(2)}, n_{1}^{(1)} n_{3}^{(2)}, n_{4}^{(2)}\right\rangle .
\end{aligned}
$$

And the cohomology groups with $U(1)$ a coefficient are

$$
\begin{aligned}
& H^{1}\left[\mathbb{Z}_{2} \times \mathbb{Z}_{2}, U(1)\right]=\mathbb{Z}_{2}^{2}, \\
& H^{2}\left[\mathbb{Z}_{2} \times \mathbb{Z}_{2}, U(1)\right]=\mathbb{Z}_{2}, \\
& H^{3}\left[\mathbb{Z}_{2} \times \mathbb{Z}_{2}, U(1)\right]=\mathbb{Z}_{2}^{3}, \\
& H^{4}\left[\mathbb{Z}_{2} \times \mathbb{Z}_{2}, U(1)\right]=\mathbb{Z}_{2}^{2}, \\
& H^{5}\left[\mathbb{Z}_{2} \times \mathbb{Z}_{2}, U(1)\right]=\mathbb{Z}_{2}^{4} .
\end{aligned}
$$

\section{a. 0D}

The classification data are $\left(n_{0}, \nu_{1}\right) \in H^{0}\left(G_{b}, \mathbb{Z}_{2}\right) \times$ $H^{1}\left[G_{b}, U(1)\right]=\mathbb{Z}_{2} \times \mathbb{Z}_{2}^{2}$. From the equation $d \nu_{1}=$ $(-1)^{\omega_{2} n_{0}}$, we see that $n_{0}=1$ is obstructed, as $\left[(-1)^{\omega_{2}}\right]=$ $\left[(-1)^{n_{1}^{(1)}} n_{1}^{(2)}\right]$ is the nontrivial cocycle in $H^{2}\left[\mathbb{Z}_{2} \times \mathbb{Z}_{2}, U(1)\right]=$ $\mathbb{Z}_{2}$. So the classification is $\mathbb{Z}_{2}^{2}$, which is the same as BSPT phases. 


\section{b. $1 D$}

The classification data are $\left(n_{0}, n_{1}, \nu_{2}\right) \in H^{0}\left(G_{b}, \mathbb{Z}_{2}\right) \times$ $H^{1}\left(G_{b}, \mathbb{Z}_{2}\right) \times H^{2}\left[G_{b}, U(1)\right]=\mathbb{Z}_{2} \times \mathbb{Z}_{2}^{2} \times \mathbb{Z}_{2}$. The equation $d n_{1}=\omega_{2} \smile n_{0}$ implies that $n_{0}=1$ is obstructed. From the equation $d \nu_{2}=(-1)^{\omega_{2} n_{1}}$, we see that $n_{1}=n_{1}^{(i)}$ is obstructed: $\left[(-1)^{\omega_{2} n_{1}^{(i)}}\right]=\left[(-1)^{n_{1}^{(i)} n_{2}^{(i)}}\right] \notin B^{3}\left[G_{b}, U(1)\right]$. The nontrivial BSPT $\nu_{2}=(-1)^{n_{1}^{(1)}} n_{1}^{(2)}$ is trivialized by $0 \mathrm{D}$ obstruction function $(-1)^{\omega_{2} n_{0}}$ with $n_{0}=1$. Therefore, there is only one trivial FSPT phase in 1D.

\section{c. 2D}

The classification data are $\left(n_{0}, n_{1}, n_{2}, \nu_{3}\right) \in$ $H^{0}\left(G_{b}, \mathbb{Z}\right) \times H^{1}\left(G_{b}, \mathbb{Z}_{2}\right) \times H^{2}\left(G_{b}, \mathbb{Z}_{2}\right) \times H^{3}\left[G_{b}, U(1)\right]=$ $\mathbb{Z} \times \mathbb{Z}_{2}^{2} \times \mathbb{Z}_{2}^{3} \times \mathbb{Z}_{2}^{3}$. The $n_{0}=1$ ( $p+i p$ superconductor $)$ state is obstructed by the equation $d n_{1}=\omega_{2} \smile n_{0}$. And all the $n_{1}$ are obstructed by the equation $d n_{2}=\omega_{2} \smile n_{1}$. The data $n_{2}=\omega_{2}$ are trivialized by 1D ASPT state $\omega_{2} \smile n_{0}$ with $n_{0}=1$. So we need only to consider $n_{2} \in \mathbb{Z}_{2}^{2}=\left\langle n_{2}^{(1)}, n_{2}^{(2)}\right\rangle$. Since $\left[(-1)^{\omega_{2} n_{2}+n_{2} n_{2}}\right]=\left[(-1)^{n_{1}^{(1)} n_{1}^{(2)} n_{2}^{(i)}}\right]$ if $n_{2}=n_{2}^{(i)}$, we conclude that all $n_{2}$ are obstructed or trivialized. For the BSPT state $\nu_{3}$, two of the three root states are trivialized by 1D ASPT $(-1)^{\omega_{2} n_{1}^{(i)}}$. Therefore, the 2D FSPT phases (invertible phases excluded) are classified by $\mathbb{Z}_{2}$, which is generated by one of the BSPT root phases.

\section{d. $3 \mathrm{D}$}

The classification data are $\left(n_{1}, n_{2}, n_{3}, \nu_{4}\right) \in H^{1}\left(G_{b}, \mathbb{Z}\right) \times$ $H^{2}\left(G_{b}, \mathbb{Z}_{2}\right) \times H^{3}\left(G_{b}, \mathbb{Z}_{2}\right) \times H^{4}\left[G_{b}, U(1)\right]=\mathbb{Z}_{1} \times \mathbb{Z}_{2}^{3} \times \mathbb{Z}_{2}^{4} \times$ $\mathbb{Z}_{2}^{2}$. The data $n_{2}=\omega_{2}$ are trivialized by $\omega_{2} \smile n_{0}$ with $n_{0}=1$. And all other $n_{2}$ are obstructed by the equation $d n_{3}=\omega_{2} n_{2}+n_{2} n_{2}$. For the $n_{3}$ data, the 1D ASPT with $\omega_{2} n_{1}^{(i)}$ trivializes the $n_{3}$ data from $\mathbb{Z}_{2}^{4}$ to $\mathbb{Z}_{2}^{2}$, which can be chosen to be $\left\langle n_{2}^{(1)} n_{1}^{(2)}, n_{1}^{(1)} n_{2}^{(2)}\right\rangle$. For the two root $n_{3}$, one can show that $\left[d \nu_{4}\right]=\left[(-1)^{\omega_{2} n_{3}+n_{3} n_{3}}\right]=$ $\left[(-1)^{n_{3}^{(1)} n_{2}^{(2)}+n_{2}^{(1)} n_{3}^{(2)}}\right]$. So the only obstruction-free and trivialization-free $n_{3}$ is $n_{3}=n_{2}^{(1)} n_{1}^{(2)}+n_{1}^{(1)} n_{2}^{(2)}$. However, from the $n_{0}=2$ trivialization Eq. (245), this $n_{3}$ is the same as and trivialized by $\omega_{2} \smile_{1} \omega_{2}=S q^{1}\left(\omega_{2}\right)=S q^{1}\left[n_{1}^{(1)} n_{1}^{(2)}\right]$. For the BSPT $\nu_{4}$, they are all trivialized by the 2D ASPT with $(-1)^{\omega_{2} n_{2}+n_{2} n_{2}}$. In conclusion, there is only one 3D FSPT trivial phase.

[1] X. Chen, Z.-C. Gu, and X.-G. Wen, Local Unitary Transformation, Long-Range Quantum Entanglement, Wave Function Renormalization, and Topological Order, Phys. Rev. B 82, 155138 (2010).

[2] Z.-C. Gu and X.-G. Wen, Tensor-Entanglement-Filtering Renormalization Approach and Symmetry-Protected Topological Order, Phys. Rev. B 80, 155131 (2009).
[3] X. Chen, Z.-C. Gu, Z.-X. Liu, and X.-G. Wen, SymmetryProtected Topological Orders in Interacting Bosonic Systems, Science 338, 1604 (2012).

[4] X. Chen, Z.-C. Gu, Z.-X. Liu, and X.-G. Wen, Symmetry Protected Topological Orders and the Group Cohomology of Their Symmetry Group, Phys. Rev. B 87, 155114 (2013).

[5] M.Z. Hasan and C. L. Kane, Colloquium: Topological Insulators, Rev. Mod. Phys. 82, 3045 (2010).

[6] X.-L. Qi and S.-C. Zhang, Topological Insulators and Superconductors, Rev. Mod. Phys. 83, 1057 (2011).

[7] F. D. M. Haldane, Nonlinear Field Theory of Large-Spin Heisenberg Antiferromagnets: Semiclassically Quantized Solitons of the One-Dimensional Easy-Axis Néel State, Phys. Rev. Lett. 50, 1153 (1983).

[8] X.-G. Wen, Construction of Bosonic Symmetry-ProtectedTrivial States and Their Topological Invariants via $g \times \operatorname{so}(\infty)$ Nonlinear $\sigma$ Models, Phys. Rev. B 91, 205101 (2015).

[9] A. Kapustin, Symmetry Protected Topological Phases, Anomalies, and Cobordisms: Beyond Group Cohomology, arXiv:1403.1467.

[10] A. Kapustin, R. Thorngren, A. Turzillo, and Z. Wang, Fermionic Symmetry Protected Topological Phases and Cobordisms, J. High Energy Phys. 12 (2015) 052.

[11] M. Levin and Z.-C. Gu, Braiding Statistics Approach to Symmetry-Protected Topological Phases, Phys. Rev. B 86, 115109 (2012).

[12] M. Cheng and Z.-C. Gu, Topological Response Theory of Abelian Symmetry-Protected Topological Phases in Two Dimensions, Phys. Rev. Lett. 112, 141602 (2014).

[13] C. Wang, C.-H. Lin, and Z.-C. Gu, Interacting Fermionic Symmetry-Protected Topological Phases in Two Dimensions, Phys. Rev. B 95, 195147 (2017).

[14] C. Wang and M. Levin, Braiding Statistics of Loop Excitations in Three Dimensions, Phys. Rev. Lett. 113, 080403 (2014).

[15] S. Jiang, A. Mesaros, and Y. Ran, Generalized Modular Transformations in $(3+1) \mathrm{D}$ Topologically Ordered Phases and Triple Linking Invariant of Loop Braiding, Phys. Rev. X 4, 031048 (2014).

[16] C. Wang and M. Levin, Topological Invariants for Gauge Theories and Symmetry-Protected Topological Phases, Phys. Rev. B 91, 165119 (2015).

[17] J. C. Wang and X.-G. Wen, Non-Abelian String and Particle Braiding in Topological Order: Modular $\operatorname{SL}(3, \mathbb{Z})$ Representation and $(3+1)$-Dimensional Twisted Gauge Theory, Phys. Rev. B 91, 035134 (2015).

[18] J. C. Wang, Z.-C. Gu, and X.-G. Wen, Field-Theory Representation of Gauge-Gravity Symmetry-Protected Topological Invariants, Group Cohomology, and Beyond, Phys. Rev. Lett. 114, 031601 (2015).

[19] C.-H. Lin and M. Levin, Loop Braiding Statistics in Exactly Soluble Three-Dimensional Lattice Models, Phys. Rev. B 92, 035115 (2015).

[20] P. Putrov, J. Wang, and S.-T. Yau, Braiding Statistics and Link Invariants of Bosonic/Fermionic Topological Quantum Matter in $2+1$ and $3+1$ Dimensions, Ann. Phys. (Amsterdam) 384C, 254 (2017). 
[21] J. Wang, K. Ohmori, P. Putrov, Y. Zheng, Z. Wan, M. Guo, H. Lin, P. Gao, and S.-T. Yau, Tunneling Topological Vacua via Extended Operators: (Spin-)TQFT Spectra and Boundary Deconfinement in Various Dimensions, Prog. Theor. Exp. Phys. 2018, 053A01 (2018).

[22] Q.-R. Wang, M. Cheng, C. Wang, and Z.-C. Gu, Topological Quantum Field Theory for Abelian Topological Phases and Loop Braiding Statistics in $(3+1)$-Dimensions, Phys. Rev. B 99, 235137 (2019).

[23] T. Lan, L. Kong, and X.-G. Wen, Classification of $(3+1) \mathrm{D}$ Bosonic Topological Orders: The Case When Pointlike Excitations Are All Bosons, Phys. Rev. X 8, 021074 (2018).

[24] T. Lan and X.-G. Wen, A Classification of $3+1 \mathrm{D}$ Bosonic Topological Orders (II): The Case When Some Point-like Excitations Are Fermions, Phys. Rev. X 9, 021005 (2019).

[25] E. Witten, Fermion Path Integrals and Topological Phases, Rev. Mod. Phys. 88, 035001 (2016).

[26] A. Vishwanath and T. Senthil, Physics of ThreeDimensional Bosonic Topological Insulators: SurfaceDeconfined Criticality and Quantized Magnetoelectric Effect, Phys. Rev. X 3, 011016 (2013).

[27] C. Wang and T. Senthil, Boson Topological Insulators: A Window into Highly Entangled Quantum Phases, Phys. Rev. B 87, 235122 (2013).

[28] X. Chen, F. J. Burnell, A. Vishwanath, and L. Fidkowski, Anomalous Symmetry Fractionalization and Surface Topological Order, Phys. Rev. X 5, 041013 (2015).

[29] C. Wang, C.-H. Lin, and M. Levin, Bulk-Boundary Correspondence for Three-Dimensional Symmetry-Protected Topological Phases, Phys. Rev. X 6, 021015 (2016).

[30] P. Bonderson, C. Nayak, and X.-L. Qi, A time-Reversal Invariant Topological Phase at the Surface of a $3 d$ Topological Insulator, J. Stat. Mech. (2013) P09016.

[31] C. Wang, A. C. Potter, and T. Senthil, Gapped Symmetry Preserving Surface State for the Electron Topological Insulator, Phys. Rev. B 88, 115137 (2013).

[32] L. Fidkowski, X. Chen, and A. Vishwanath, Non-Abelian Topological Order on the Surface of a $3 d$ Topological Superconductor from an Exactly Solved Model, Phys. Rev. X 3, 041016 (2013).

[33] X. Chen, L. Fidkowski, and A. Vishwanath, Symmetry Enforced Non-Abelian Topological Order at the Surface of a Topological Insulator, Phys. Rev. B 89, 165132 (2014).

[34] C. Wang and T. Senthil, Interacting Fermionic Topological Insulators/Superconductors in Three Dimensions, Phys. Rev. B 89, 195124 (2014).

[35] M. A. Metlitski, L. Fidkowski, X. Chen, and A. Vishwanath, Interaction Effects on 3D Topological Superconductors: Surface Topological Order from Vortex Condensation, the 16 Fold Way and Fermionic Kramers Doublets, arXiv: 1406.3032.

[36] M. A. Metlitski, C. L. Kane, and M. P. A. Fisher, SymmetryRespecting Topologically Ordered Surface Phase of Three-Dimensional Electron Topological Insulators, Phys. Rev. B 92, 125111 (2015).

[37] L. Fidkowski, A. Vishwanath, and M. A. Metlitski, Surface Topological Order and a New 't Hooft Anomaly of Interaction Enabled 3 + 1D Fermion SPTs, arXiv:1804.08628.

[38] A. P. Schnyder, S. Ryu, A. Furusaki, and A. W. W. Ludwig, Classification of Topological Insulators and Superconduc- tors in Three Spatial Dimensions, Phys. Rev. B 78, 195125 (2008).

[39] A. Kitaev, Periodic Table for Topological Insulators and Superconductors, AIP Conf. Proc. 1134, 22 (2009).

[40] S. Ryu, A. P. Schnyder, A. Furusaki, and A. W. W. Ludwig, Topological Insulators and Superconductors: Tenfold Way and Dimensional Hierarchy, New J. Phys. 12, 065010 (2010).

[41] X.-G. Wen, Symmetry-Protected Topological Phases in Noninteracting Fermion Systems, Phys. Rev. B 85 , 085103 (2012).

[42] L. Fidkowski and A. Kitaev, Effects of Interactions on the Topological Classification of Free Fermion Systems, Phys. Rev. B 81, 134509 (2010).

[43] L. Fidkowski and A. Kitaev, Topological Phases of Fermions in One Dimension, Phys. Rev. B 83, 075103 (2011).

[44] X.-L. Qi, A New Class of (2+1)-Dimensional Topological Superconductors with $\mathbb{Z}_{8}$ Topological Classification, New J. Phys. 15, 065002 (2013).

[45] H. Yao and S. Ryu, Interaction Effect on Topological Classification of Superconductors in Two Dimensions, Phys. Rev. B 88, 064507 (2013).

[46] S. Ryu and S.-C. Zhang, Interacting Topological Phases and Modular Invariance, Phys. Rev. B 85, 245132 (2012).

[47] Z.-C. Gu and M. Levin, Effect of Interactions on TwoDimensional Fermionic Symmetry-Protected Topological Phases with $Z_{2}$ Symmetry, Phys. Rev. B 89, 201113(R) (2014).

[48] Y.-Z. You and C. Xu, Symmetry-Protected Topological States of Interacting Fermions and Bosons, Phys. Rev. B 90, 245120 (2014).

[49] T. Morimoto, A. Furusaki, and C. Mudry, Breakdown of the Topological Classification $\mathbb{Z}$ for Gapped Phases of Noninteracting Fermions by Quartic Interactions, Phys. Rev. B 92, 125104 (2015).

[50] C. Wang, A. C. Potter, and T. Senthil, Classification of Interacting Electronic Topological Insulators in Three Dimensions, Science 343, 629 (2014).

[51] T. Neupert, C. Chamon, C. Mudry, and R. Thomale, Wire Deconstructionism of Two-Dimensional Topological Phases, Phys. Rev. B 90, 205101 (2014).

[52] Z.-C. Gu and X.-G. Wen, Symmetry-Protected Topological Orders for Interacting Fermions: Fermionic Topological Nonlinear $\sigma$ Models and a Special Group Supercohomology Theory, Phys. Rev. B 90, 115141 (2014).

[53] Q.-R. Wang and Z.-C. Gu, Towards a Complete Classification of Symmetry-Protected Topological Phases for Interacting Fermions in Three Dimensions and a General Group Supercohomology Theory, Phys. Rev. X 8, 011055 (2018).

[54] X. Chen, Z.-C. Gu, and X.-G. Wen, Classification of Gapped Symmetric Phases in One-Dimensional Spin Systems, Phys. Rev. B 83, 035107 (2011).

[55] M. Cheng, Z. Bi, Y.-Z. You, and Z.-C. Gu, Towards a Complete Classification of Symmetry-Protected Phases for Interacting Fermions in Two Dimensions, Phys. Rev. B 97, 205109 (2018).

[56] C. Wang, Braiding Statistics and Classification of TwoDimensional Charge-2m Superconductors, Phys. Rev. B 94, 085130 (2016). 
[57] D. Gaiotto and A. Kapustin, Spin TQFTs and Fermionic Phases of Matter, Int. J. Mod. Phys. A 31, 1645044 (2016).

[58] D. S. Freed, Short-Range Entanglement and Invertible Field Theories, arXiv:1406.7278.

[59] L. Bhardwaj, D. Gaiotto, and A. Kapustin, State Sum Constructions of Spin-TFTs and String Net Constructions of Fermionic Phases of Matter, J. High Energy Phys. 04 (2017) 096.

[60] D. S. Freed and M. J. Hopkins, Reflection Positivity and Invertible Topological Phases, arXiv:1604.06527.

[61] G. Brumfiel and J. Morgan, The Pontrjagin Dual of 3-Dimensional Spin Bordism, arXiv:1612.02860.

[62] A. Kapustin and R. Thorngren, Fermionic SPT Phases in Higher Dimensions and Bosonization, J. High Energy Phys. 10 (2017) 080.

[63] G. Brumfiel and J. Morgan, The Pontrjagin Dual of 4-Dimensional Spin Bordism, arXiv:1803.08147.

[64] Y.-A. Chen, A. Kapustin, A. Turzillo, and M. You, Free and Interacting Short-Range Entangled Phases of Fermions: Beyond the Tenfold Way, Phys. Rev. B 100, 195128 (2019).

[65] N. E. Steenrod, Products of Cocycles and Extensions of Mappings, Ann. Math. 48, 290 (1947).

[66] Q.-R. Wang, Y. Qi, and Z.-C. Gu, Anomalous Symmetry Protected Topological States in Interacting Fermion Systems, Phys. Rev. Lett. 123, 207003 (2019).

[67] C. Wang (private communication).

[68] J.-R. Zhou, Q.-R. Wang, C. Wang, and Z.-C. Gu, NonAbelian Three-Loop Braiding Statistics for 3D Fermionic Topological Phases, arXiv:1912.13505.

[69] Considering the entanglement density matrix $\rho_{A}$ for a SPT state in region $A, \rho_{A}$ may act on a subspace of the Hilbert space in region $A$, and the subspace is called the support space $\tilde{V}_{A}$ of region $A$.

[70] In principle, we can also add an arbitrary phase factor into the above move. However, since such a phase factor must be a symmetric $U(1)$-valued function of group elements $g_{0}, g_{1}$, and $g_{2}$, it can always be removed by the basis redefinition Eq. (45).

[71] In principle, we can also add an arbitrary phase factor into the above move. However, since such a phase factor must be a $U(1)$-valued function of group elements $g_{0}, g_{1}, g_{2}$, and $g_{3}$, it can always be removed by the basis redefinition Eq. (53).

[72] Z.-C. Gu, Z. Wang, and X.-G. Wen, Classification of TwoDimensional Fermionic and Bosonic Topological Orders, Phys. Rev. B 91, 125149 (2015).

[73] We assume $G_{b}$ to be a finite group in this paper.
[74] N. Tarantino and L. Fidkowski, Discrete Spin Structures and Commuting Projector Models for Two-Dimensional Fermionic Symmetry-Protected Topological Phases, Phys. Rev. B 94, 115115 (2016).

[75] B. Ware, J. H. Son, M. Cheng, R. V. Mishmash, J. Alicea, and B. Bauer, Ising Anyons in Frustration-Free MajoranaDimer Models, Phys. Rev. B 94, 115127 (2016).

[76] Z. Wang, S.-Q. Ning, and X. Chen, Exactly Solvable Model for Two-Dimensional Topological Superconductors, Phys. Rev. B 98, 094502 (2018).

[77] J. Brundan and A.P. Ellis, Monoidal Supercategories, Commun. Math. Phys. 351, 1045 (2017).

[78] R. Usher, Fermionic 6 j-Symbols in Superfusion Categories, arXiv:1606.03466.

[79] For any vertex $i$, there are always two Majorana fermions having a second type of pairing with another site. So the fermion parity changes such as $(-1)^{\omega_{2}\left(g, g_{i}\right)}$ appear in pairs. For the third type of pairing, the number of $A-A$ and $B-B$ pairing is always even for a closed chain. So the total fermion parity is fixed.

[80] A. Kapustin and L. Fidkowski, Local Commuting Projector Hamiltonians and the Quantum Hall Effect, Commun. Math. Phys. 373, 763 (2020).

[81] A. Yu. Kitaev, Unpaired Majorana Fermions in Quantum Wires, Phys. Usp. 44, 131 (2001).

[82] C. Meng, private communications.

[83] H. Song, S.-J. Huang, L. Fu, and M. Hermele, Topological Phases Protected by Point Group Symmetry, Phys. Rev. X 7 , 011020 (2017).

[84] R. Z. Goldstein and E. C. Turner, A Formula for StiefelWhitney Homology Classes, Proc. Am. Math. Soc. 58, 339 (1976).

[85] M. Cheng, N. Tantivasadakarn, and C. Wang, Loop Braiding Statistics and Interacting Fermionic SymmetryProtected Topological Phases in Three Dimensions, Phys. Rev. X 8, 011054 (2018).

[86] N. Tantivasadakarn, Dimensional Reduction and Topological Invariants of Symmetry-Protected Topological Phases, Phys. Rev. B 96, 195101 (2017).

[87] This situation is equivalent to $\#_{2}\left(N_{0}\right) \geq \#_{2}\left(N_{i}\right) \geq 1$, where $\#_{2}(x)$ denotes the number of 2's in the prime factorization of integer $x$.

[88] One may need some identities such as $\operatorname{gcd}\left(2 N_{0}, N_{i j}\right)=$ $N_{0 i j} \cdot \operatorname{gcd}\left(2, N_{i j} / N_{0 i j}\right)$ when $N_{0}$ is even.

[89] Q.-R. Wang and Z.-C. Gu (to be published).

[90] A. Kitaev, http://www.ipam.ucla.edu/abstract/?tid=12389\& pcode $=$ STQ2015. 